\title{
Autism spectrum disorders in high functioning adolescents
}

Citation for published version (APA):

Barendse, E. M. (2017). Autism spectrum disorders in high functioning adolescents: diagnostic considerations (AHA). [Doctoral Thesis, Maastricht University]. Maastricht University. https://doi.org/10.26481/dis.20170907eb

Document status and date:

Published: 01/01/2017

DOI:

10.26481/dis.20170907eb

Document Version:

Publisher's PDF, also known as Version of record

\section{Please check the document version of this publication:}

- A submitted manuscript is the version of the article upon submission and before peer-review. There can be important differences between the submitted version and the official published version of record.

People interested in the research are advised to contact the author for the final version of the publication, or visit the DOI to the publisher's website.

- The final author version and the galley proof are versions of the publication after peer review.

- The final published version features the final layout of the paper including the volume, issue and page numbers.

Link to publication

\footnotetext{
General rights rights.

- You may freely distribute the URL identifying the publication in the public portal. please follow below link for the End User Agreement:

www.umlib.nl/taverne-license

Take down policy

If you believe that this document breaches copyright please contact us at:

repository@maastrichtuniversity.nl

providing details and we will investigate your claim.
}

Copyright and moral rights for the publications made accessible in the public portal are retained by the authors and/or other copyright owners and it is a condition of accessing publications that users recognise and abide by the legal requirements associated with these

- Users may download and print one copy of any publication from the public portal for the purpose of private study or research.

- You may not further distribute the material or use it for any profit-making activity or commercial gain

If the publication is distributed under the terms of Article $25 \mathrm{fa}$ of the Dutch Copyright Act, indicated by the "Taverne" license above, 


\title{
AUTISM SPECTRUM DISORDERS IN HIGH FUNCTIONING ADOLESCENTS; DIAGNOSTIC CONSIDERATIONS
}

(AHA)

\author{
Evelien Maria Barendse
}


This research is funded by

\section{ISBN}

978-94-6284-117-8

\section{Design/lay-out}

Promotie In Zicht, Arnhem

\section{Print}

Ipskamp Printing, Enschede

Copyright (C) Evelien M. Barendse, 2017

All rights reserved. No parts of this publication may be reported or transmitted, in any form or by any means, without permission in writing of the authors, or, when appropriate, from the publishers of the publication. 


\section{AUTISM SPECTRUM DISORDERS IN HIGH FUNCTIONING ADOLESCENTS; DIAGNOSTIC CONSIDERATIONS}

(AHA)

\section{Proefschrift}

Ter verkrijging van de graad van doctor aan de Universiteit Maastricht, op gezag van de Rector Magnificus, Prof. dr. Rianne M. Letschert, volgens het besluit van het College van Decanen, in het openbaar te verdedigen op donderdag 7 september om 12:00 uur

door

Evelien Maria Barendse geboren op 10 oktober 1980 te Wageningen 


\section{Promotoren}

Prof. dr. A.P. Aldenkamp

Prof. dr. R.P.C. Kessels (Radboud Universiteit, Nijmegen)

\section{Copromotoren}

Dr. M.P.H. Hendriks (Radboud Universiteit, Nijmegen)

Dr. J.F.A. Jansen

\section{Beoordelingscommissie}

Prof. dr. R.W.H.M. Ponds (voorzitter)

Prof. dr. J.I.M. Egger (Radboud Universiteit, Nijmegen)

Prof. dr. H.M. Geurts (Universiteit van Amsterdam)

Dr. J. Nicolai

Prof. dr. J.E. Wildberger 


\section{Contents}

$\begin{array}{lll}\text { Chapter } 1 & \text { General introduction and outline } & 7\end{array}$

Chapter 2 Using the Achenbach Screening Instruments in high-functioning 23 adolescents with an autism spectrum disorder

Chapter 3 Social Behaviour and Social Cognition in high-functioning adolescents with an Autism Spectrum Disorder (ASD): two sides of the same coin?

Chapter 4 The applicability of standardized measures of executive 65 functioning in high-functioning adolescents with an autism spectrum disorder (ASD)

Chapter 5 Review: Working memory deficits in high-functioning adolescents with autism spectrum disorders: neuropsychological and neuroimaging correlates

Chapter 6 Working memory network alterations in high-functioning 107 adolescents with an autism spectrum disorder

Chapter 7 Brain resting-state networks in adolescents with highfunctioning autism: analysis of spatial connectivity and temporal neurodynamics

Chapter 8 Altered neurotransmitter metabolism in adolescents with highfunctioning autism

Chapter 9 General discussion

Summary | Samenvatting $\quad 165$

Societal relevance 173

$\begin{array}{ll}\text { Abbreviations } & 177\end{array}$

$\begin{array}{lr}\text { References } & 179\end{array}$

Dankwoord 199

Curriculum Vitae 203

Donders Gratuate School for Cognitive Neuroscience Series 206 

1

GENERAL INTRODUCTION AND OUTLINE 



\section{Autism Spectrum Disorders}

"When I was little and somebody asked me: 'Don't you want a cookie?' I said 'no' because I wanted a cookie. Now I know that I should say 'yes' to that question." [participant diagnosed with an ASD]

\section{Historical perspective}

The term "autism" (derived from the Greek word "autóc" meaning "self") has been used from the early 1900's on and was introduced by the Swiss psychiatrist Paul Eugen Bleuler. In his work on schizophrenia, he used this term to describe persons who definite withdrew themselves from the external world. In 1943, the Austrian-American psychiatrist Leo Kanner used and specified this term to 'early infantile autism' to describe a common pattern of behaviour that he observed in 11 disturbed children. These children (8 boys and 3 girls)

all showed symptoms of extreme alones from the beginning

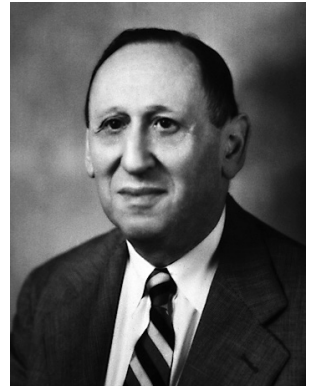

Dr. Leo Kanner of life and an anxiously obsessive desire for the preservation of sameness. Kanner (1943) concluded that "[..]these children have come into the world with an innate inability to form the usual, biologically provided affected contact with people, just as other children come into the world with physical and intellectual handicaps" (p. 250). In a later paper, Kanner (1965) acknowledged that the definition as proposed by Bleuler did not fit the status of his patients very good, as withdrawal implies a removal of oneself of previous participation. The children he saw never really participated in the external social world. However, as he was unable to find a more suitable definition for this 'new' condition, he decided to use the term autism.

Around the same time as Kanner's first publication on autism, Hans Asperger, a child practitioner from Vienna, published a paper titled "Die Autistischen Psychopathen im Kindesalter" (Asperger, 1943). He also borrowed the term 'autismus' as introduced by Bleuler and specified it further by adding the term 'psychopathy', which he used in the technical sense of an abnormality of personality. In this, in German written, article, Asperger gave clinical descriptions of four boys, who, despite their differences in intellect, personality, the severity of the disorder etc., presented common defiant behaviour patterns

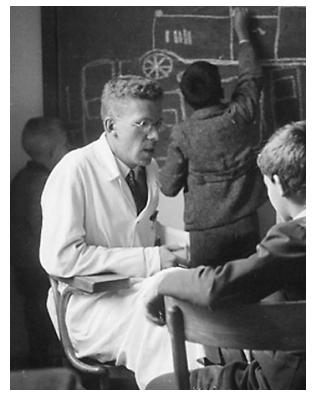

Dr. Hans Asperger 
similar to those described by Kanner. All four boys showed social impairments, communication difficulties, and repetitive activities and resistance to change. But, contrary to Kanner's patient descriptions, these symptoms were never recognized in infancy and usually not before the third year of life or later. Asperger furthermore described that these 'Autistischen Psychopathen' beside their impairments, also had certain special skills which gave them an expression of marked eccentricity. He further described that as these children were so different from their peers, they may be mercilessly bullied at school. Especially, as Asperger nicely put it, because these children often have a particularly good view and unerring ridicule for other peoples' particularities, and have a lack of ability to understand the social rules governing social behaviour.

In 1980, autism was first mentioned as a distinct category in the Diagnostic and Statistical Manual of Mental Disorders, third edition (DSM-III). However, 'infantile autism' was designated as the only form of autism and solely six characteristics were listed; an individual having to possess each of which to be labelled 'autistic' (American Psychiatric Association, 1980). Throughout the years, subsequent studies showed that autism is not so much a categorical disorder, but is a dimensional spectrum (Wing, 1996; Wing \& Potter, 2002), with a large heterogeneity in phenotype, severity, intellectual abilities, and type and frequency of symptoms. In addition, symptoms can change within one person over the course of development. As a consequence, the DSM-IV added several subtypes to the category 'pervasive developmental disorders' including autism disorder, Asperger syndrome, and pervasive developmental disorder not otherwise specified. Also additional symptoms and more subtle features have been included in the definition of the autism spectrum disorders (ASD) in the DSM-IV (see Table 1). Another distinction that is currently made, although not part of the diagnostic classification, is low-functioning autism and high-functioning autism. Low-functioning individuals with ASD are generally considered to have an IQ below 70, whereas high-functioning individuals with ASD tend to have an IQ above 70 and near normal language skills. Throughout this manuscript, only the clinical and research findings of high- functioning individuals will be discussed.

\section{Neuropsychological findings}

Research shows that a normal or near-normal IQ is necessary but not sufficient for better outcomes in adaptive functioning. Social skills and autism symptomatology can occur with equal severity in low-functioning individuals as in high-functioning individuals. This discrepancy between intelligence and social functioning is especially prominent among the higher-ability individuals (Joseph, 2011). Moreover, individuals 
with ASD often present a highly variable cognitive profile; with some functions impaired, while others are spared or even superior.

\section{Table 1 Criteria for Autistic Disorder according to the DSM-IV-TR}

A. A total of six (or more) of 1,2 , and 3

1) Qualitative impairment in social interaction, as manifested by at least two of the following:

a) Marked impairment in the use of multiple nonverbal behaviours such as eye-to-eye gaze, facial expression, body postures, and gestures to regulate social interaction

b) Failure to develop peer relationships appropriate to developmental level

c) A lack of spontaneous seeking to share enjoyment, interests or achievements with other people (e.g. by lack of showing, bringing or pointing out objects of interest)

d) Lack of social or emotional reciprocity

2) Qualitative impairments in communication as manifested by at least one of the following:

a) Delay in, or total lack of, the development of spoken language (not accompanied by an attempt to compensate through alternative modes of communication such as gesture or mime)

b) In individuals with adequate speech, marked impairment in the ability to initiate or sustain a conversation with others

c) Stereotyped and repetitive use of language or idiosyncratic language

d) Lack of varied, spontaneous make-believe play or social imitative play appropriate to developmental level

3) Restricted repetitive and stereotyped patterns of behaviour, interests, and activities, as manifested by at least one of the following:

a) Encompassing preoccupation with one or more stereotyped and restricted patterns of interests that is abnormal either in intensity or focus

b) Apparently inflexible adherence to specific, non-functional routines or rituals

c) Stereotyped and repetitive motor mannerisms (e.g. hand or finger flapping or twisting, or complex whole-body movements)

d) Persistent preoccupation with parts of objects

B. Delays or abnormal functioning in at least one of the following areas, with onset before the age of 3 years: 1) social interaction, 2) language as used in social communication or 3) symbolic or imaginative play.

C. The disturbance is not better accounted for by Rett's Disorder or Childhood Disintegrative Disorder. 


\section{Language}

Communication difficulties are one of the three core symptoms of ASD and are seen throughout the spectrum. Many individuals with ASD show a language delay early in development and continue to have communication difficulties throughout the lifespan. Even the most verbally fluent individuals with ASD experience clear difficulties with the social and communicative aspects of language. Problems arise in prosody (e.g. speaking too loud, t0o fast, too monotonous), semantic (e.g. use of neologisms, echolalia), and pragmatic domains of language. Many have problems in initiating a conversation, maintaining the topic of the conversation and considering the perspective of the listener. Problems also arise in understanding figurative language, inferences, metaphors, subtle nonverbal cues, and (subtle) humour and sarcasm. The tendency to take things literally is also shown in a pedantic, over-exact, comprehension and production style, especially in higher-functioning children and adolescents (for a good review see: Kelley, 2011).

\section{Attention and inhibition}

Individuals with ASD often have difficulty shifting and refocusing their attention and are likely to miss quickly occurring environmental information that requires rapid shifts of attention. There is also evidence for both overly narrow and overly broad spatial attention; individuals with ASD may have a superior attention to details, but at the same time also have problems filtering out irrelevant information from the environment. Travers and colleagues (Travers, Klinger, \& Klinger, 2011) propose that an inability to flexibly switch between a narrow and broad spotlight of attention, may explain this phenomenon. No global problems in selective attention have been reported (Travers et al., 2011). There is also evidence for preserved abilities in response inhibition, indicating that some of these processes are intact, or that reorganization and compensatory behaviours make them appear that way. Impairments have been found in the ability to inhibit prepotent motor responses (for review see: O'Hearn, Asato, Ordaz, \& Luna, 2008).

\section{Memory}

Also in the memory domain, individuals with ASD show spared and impaired abilities. Especially high-functioning individuals with ASD frequently have excellent rote memory for facts of topics of interest (Boucher \& Mayes, 2011; F. Happe \& Frith, 1996). Short-term memory, cued recall and nondeclarative memory are predominantly intact as well. Memory impairments are generally found in visuospatial working memory and in tasks that are episodic in nature, particularly on tasks that do not allow the use of compensatory strategies involving semantic memory or the perceptual representation system (Ben Shalom, 2003; Boucher \& Mayes, 2011). Source memory is only found intact where contextual information is available within 
the immediate environment, but is impaired in tasks where contextual information must be recalled. Memory for information with social or emotional connotations, whether involving self or others, is generally poor, both across nondeclarative as declarative forms of learning (Boucher \& Mayes, 2011).

\section{Executive functions}

Traditionally, executive functions are described as a group of higher cognitive functions necessary for the regulation of voluntary planned behaviour and the inhibition of automatic task-inappropriate responses (O'Hearn et al., 2008). Pennington and Ozonoff (1996) suggested five domains of executive functions: inhibition, working memory, planning, cognitive flexibility and fluency. Research on executive functions in highfunctioning individuals with ASD shows behavioural problems in all these domains (e.g. : Corbett, Constantine, Hendren, Rocke, \& Ozonoff, 2009; Eigsti, 2011; Geurts, Verté, Oosterlaan, Roeyers, \& Sergeant, 2004; Robinson, Goddard, Dritschel, Wisley, \& Howlin, 2009; Steele, Minshew, Luna, \& Sweeney, 2007; Verté, Geurts, Roeyers, Oosterlaan, \& Sergeant, 2006a; Zinke et al., 2010), however, results differ between studies. In part, these mixed results are due to the fact that executive functioning is an umbrella term and is therefore interpreted in different ways across different research designs. These mixed results can also be explained by group differences and the compensation strategies high-functioning individuals with ASD often adopt in structured (test) settings.

\section{Social perception and social cognition}

Social impairments are considered to be the most prominent and persistent of the three core symptoms of ASD (i.e. social impairments, communication difficulties, and repetitive interests and activities). Individuals with ASD have deficits in various aspects of social perception and social cognition, and these deficits are independent of age and developmental level (see chapter 3). For example, difficulties have been found in the ability to impute and understand the mental states of others, and recognize that these states may differ from your own (Theory of Mind). Other deficits arise in the use of communicative gestures, in imitating facial and body movements and immediate and delayed imitation of actions on objects. There are often problems in making good quality eye contact, in initiating and participating in joint attention, in recognizing facial identities, and in perceiving, labelling and understanding facial expressions (for extensive review see: South, Schultz, \& Ozonoff, 2011). Although it has been proposed that insufficient motivation for social engagement would lead to less social experience, which in turn may impact social perceptual and social cognitive skill development (Schultz, 2005), this model remains to be proven. 


\section{Motor development}

Problems in motor coordination are seen throughout the whole spectrum and have been described since the early years by Asperger (Asperger, 1943; Wing, 1981), but are not part of the diagnostic criteria. Besides the stereotype movements of the body and limbs, motor abnormalities are observed in basic aspects of motor control such as gait, posture, coordination and tone as well as difficulties in performing higher order skills including imitation and pantomime of complex gestures. Individuals with autism show relatively poorer performance in spontaneous and automatic imitation (mimicry) than in structured imitation, and they are less deficient in imitating goal-directed actions on objects than gestural and facial movements (Wodka \& Mostofsky, 2011).

\section{Sensory functions}

In high -functioning individuals with ASD, both superior and impairments have been reported in all sensory processing abilities, including olfactory, visual, auditory and tactile functions (Kenet, 2011). They may be hypersensitive to incoming stimuli and show strong reactions to loud sounds, bright lights, the touch of others' or one's own clothes, and strong smells. At the same time, they can be hyposensitive to such stimuli and may even show a reduced reaction to pain. Individuals with ASD also often show "sensory-seeking" behaviours including a fascination with spinning or brightly coloured objects, and intense looking at flickering light, specific objects, or people (C. Pellicano, 2013). Although both Kanner (1943) and Asperger (1943) wrote these kind of symptoms in their initial descriptions, it is only since the release of the DSM-5 in 2013 that these symptoms are part of the clinical description of ASD (see Table 2).

\section{Autism Spectrum Disorders today}

Since the original publication of 'Autistic Disturbances in Affective Contact' (Kanner, 1943) much research on the aetiology of ASD has taken place, but until now without conclusive results. There is overall consensus that ASD is highly genetic (for review see: Abrahams \& Geschwind, 2008) ${ }^{2} \ddot{y}$ and several autism susceptibility genes and gene variations have been identified. Further progress has been made in the research on the neurobiology and neurochemistry of ASD, where abnormalities in cerebellum, brainstem, hippocampus, amygdala, frontal lobes and other neo- and subcortical structures and neurotransmitter levels have been found (Abrahams \& Geschwind, 2008; Carper \& Courchesne, 2000; Casanova et al., 2006; Courchesne, Carper, \& Akshoomoff, 2003; Hadjikhani, Joseph, Snyder, \& Tager-Flusberg, 2006; Hardan, Muddasani, Vemulapalli, Keshavan, \& Minshew, 2006; Herbert et al., 2003; Lam, 
Aman, \& Arnold, 2006; M Langen et al., 2009; McAlonan et al., 2002) but due to the great heterogeneity of the disorder it is hard to relate these laboratory findings to every day behaviour.

Table 2 Diagnostic criteria for Autism Spectrum Disorder according to the DSM-5

A. Persistent deficits in social communication and social interaction across multiple contexts, as manifested by the following, currently or by history (examples are illustrative, not exhaustive):

1) Deficits in social-emotional reciprocity, ranging, for example, from abnormal social approach and failure of normal back-and-forth conversation; to reduced sharing of interests, emotions, or affect; to failure to initiate or respond to social interactions.

2) Deficits in nonverbal communicative behaviours used for social interaction, ranging, for example from poorly integrated verbal and nonverbal communication; to abnormalities in eye contact and body language or deficits in understanding and use of gestures; to a total lack of facial expressions and nonverbal communication.

3) Deficits in developing, maintaining, and understanding relationships, ranging, for example, from difficulties adjusting behaviour to suit various social contexts; to difficulties in sharing imaginative play or in making friends; to absence of interest in peers.

B. Restricted, repetitive patterns of behaviour, interests, or activities, as manifested by at least two of the following, currently or by history (examples are illustrative, not exhaustive):

1) Stereotyped or repetitive motor movements, use of objects, or speech (e.g. simple motor stereotypes, lining up toys or flipping objects, echolalia, idiosyncratic phrases).

2) Insistence on sameness, inflexible adherence to routines, or ritualized patterns or nonverbal behaviour (e.g. extreme distress at small changes, difficulties with transitions, rigid thinking patterns, greeting rituals, need to take same route or eat same food every day).

3) Highly restricted, fixated interests that are abnormal in intensity or focus (e.g. strong attachment to or preoccupation with unusual objects, excessively circumscribed or perseverative interests).

4) Hyper- or hyporeactivity to sensory input or unusual interests in sensory aspects of the environment (e.g. apparent indifference to pain/temperature, adverse response to specific sounds or textures, excessive smelling or touching of objects, visual fascination with lights or movement).

Specify current severity of $A$ and $B$ (table $2 b$ ) 


\section{Table 2 Continued}

C. Symptoms must be present in the early developmental period (but may not become fully manifest until social demands exceed limited capacities, or may be masked by learned strategies later in life).

D. Symptoms cause clinically significant impairment in social, occupational, or other important areas of current functioning.

E. These disturbances are not better explained by intellectual disability (intellectual developmental disorder) or global developmental delay. Intellectual disability and autism spectrum disorder frequently co-occurs; to make comorbid diagnoses of autism spectrum disorder and intellectual disability, social communication should be below that expected for general developmental level.

Specify if:

With or without accompanying intellectual impairment

With or without accompanying language impairment

Associated with a known medical or genetic condition or environmental factor Associated with another neurodevelopmental, mental, or behavioural disorder With catatonia.

Table 2b Severity levels for Autism Spectrum Disorder according to the DSM-5

\begin{tabular}{|c|c|c|}
\hline Severity level & Social communication & $\begin{array}{l}\text { Restricted, } \\
\text { repetitive behaviours }\end{array}$ \\
\hline $\begin{array}{l}\text { Level } 3 \\
\text { "Requiring } \\
\text { very } \\
\text { substantial } \\
\text { support" }\end{array}$ & $\begin{array}{l}\text { Severe deficits in verbal and } \\
\text { nonverbal social communication } \\
\text { skills cause severe impairments in } \\
\text { functioning, very limited initiation } \\
\text { of social interactions, and minimal } \\
\text { response to social overtures from } \\
\text { others. For example, a person with } \\
\text { few words of intelligible speech } \\
\text { who rarely initiates interaction, and } \\
\text { when (s)he does, makes unusual } \\
\text { approaches to meet needs only and } \\
\text { responds to only very direct social } \\
\text { approaches. }\end{array}$ & $\begin{array}{l}\text { Inflexibility of behaviour, extreme } \\
\text { difficulty coping with change, } \\
\text { or other restricted/repetitive } \\
\text { behaviours markedly interfere } \\
\text { with functioning in all spheres. } \\
\text { Great distress/difficulty changing } \\
\text { focus or action. }\end{array}$ \\
\hline
\end{tabular}


Table $\mathbf{2 b}$ Continued

\begin{tabular}{|c|c|c|}
\hline Severity level & Social communication & $\begin{array}{l}\text { Restricted, } \\
\text { repetitive behaviours }\end{array}$ \\
\hline $\begin{array}{l}\text { Level } 2 \\
\text { "Requiring } \\
\text { substantial } \\
\text { support" }\end{array}$ & $\begin{array}{l}\text { Marked deficits in verbal and } \\
\text { nonverbal social communication } \\
\text { skills; social impairments apparent } \\
\text { even with supports in place; limited } \\
\text { initiation of social interactions; and } \\
\text { reduced or abnormal responses to } \\
\text { social overtures from others. For } \\
\text { example, a person who speaks } \\
\text { simple sentences, whose interactions } \\
\text { is limited to narrow special interests, } \\
\text { and who has markedly odd } \\
\text { nonverbal communication. }\end{array}$ & $\begin{array}{l}\text { Inflexibility of behaviour, difficulty } \\
\text { coping with change, or other } \\
\text { restricted/repetitive behaviours } \\
\text { appear frequently enough to be } \\
\text { obvious to the casual observer } \\
\text { and interfere with functioning in } \\
\text { a variety of contexts. Distress } \\
\text { and/or difficulty changing focus } \\
\text { or action. }\end{array}$ \\
\hline $\begin{array}{l}\text { Level } 1 \\
\text { "Requiring } \\
\text { support" }\end{array}$ & $\begin{array}{l}\text { Without supports in place, deficits } \\
\text { in social communication cause } \\
\text { noticeable impairments. Difficulty } \\
\text { in initiating social interactions, } \\
\text { and clear examples of atypical or } \\
\text { unsuccessful response to social } \\
\text { overtures of others. May appear to } \\
\text { have decreased interests in social } \\
\text { interactions. For example, a person } \\
\text { who is able to speak in full sentences } \\
\text { and engages in communication but } \\
\text { whose to-and-fro conversation with } \\
\text { others fail, and whose attempts to } \\
\text { make friends are odd and typically } \\
\text { unsuccessful. }\end{array}$ & $\begin{array}{l}\text { Inflexibility of behaviour causes } \\
\text { significant interference with } \\
\text { functioning in one or more } \\
\text { contexts. Difficulty switching } \\
\text { between activities. Problems } \\
\text { of organization and planning } \\
\text { hamper independence. }\end{array}$ \\
\hline
\end{tabular}

There has also been much research on the aetiology of ASD at a cognitive level, and in the past decades three main key cognitive theories have been proposed: the Theory of Mind deficit hypothesis (also referred to as mentalizing theory) (S Baron-Cohen, Leslie, \& Frith, 1985), the Weak Central Coherence account (Frith, 1989), and the Executive dysfunction theory (Hill, 2004), which all will be further discussed in chapter 5. Although these theories focus on certain behavioural symptoms, neither of them can give an explanation for the broad spectrum (social and non-social) of symptoms found in ASD. Thus, many questions regarding the link 
between everyday behaviour, cognition and brain functioning in ASD remain to be answered, and more research is still needed to explain the specific combination of symptoms found in this complex disorder.

All this genetic, neuroscience and cognitive research has led to the supposition that ASD is a neurodevelopmental disorder. This is also reflected in the new DSM-5, where ASD is grouped under the 'neurodevelopmental disorders' and no longer under the category 'pervasive developmental disorders'. However, because of the inconsistency in results between studies and the still unknown aetiology, in clinical practice ASD diagnoses are still made on basis of behaviour. According to the (in our study and in the Netherlands still clinically used) DSM-IV (Table 1), ASD signs must be present at three years of age and must limit or impair everyday functioning. Diagnostic instruments, such as the Autism Diagnostic Interview (ADI) and the Autism Diagnostic Observation Schedule (ADOS), are based on an intense clinical interview of the caregivers and history taking (focusing on early childhood) and on currently observed behaviour. The parent-interviews may be used retrospectively, whereby early abnormalities may be recognized in cases were diagnosis occurs later. At present autism is usually diagnosed in the third of fourth year of life and later for atypical or mild cases (F. Happe \& Frith, 1996; van Oosten, Oosterhoff, \& Kan, 2011). Unfortunately, in high-functioning children, who often have more subtle autism features, diagnoses may be missed until secondary school when problems with learning and social interaction with peers begin to become apparent (Wing, 1997).

In the Netherlands a growing number of high average intelligent adolescents with ASD is in the need for special secondary education. These adolescents often experience many problems in their everyday life, and executive functioning problems are commonly recognized by parents, caregivers and teachers. Diagnostic interviews, observations, and neuropsychological tests, however, are challenging to apply in these high functioning adolescents with ASD. Because of their ability to masquerade their symptoms, especially in clinical or laboratory settings, it is very difficult to diagnose and inventory their problems (Rump, Giovannelli, Minshew, \& Strauss, 2009; Salter, Seigal, Claxton, Lawrence, \& Skuse, 2008). making a good diagnosis is extra important at this age while, although the social difficulties are independent of age and developmental level, the related psychosocial stress may disproportionally increase during adolescence (Nicpon, Doobay, \& Assouline, 2010; E. Pellicano, 2010b). This is especially noticeable in high-functioning adolescents with ASD, as they more than their low-functioning counterparts, seek and initiate social interaction with peers (Bauminger, Shulman, \& Agam, 2003; Hauck, Fein, Waterhouse, \& Feinstein, 1995). Despite the need for and known difficulties in diagnosing these high-functioning adolescents, in clinical practice and scientific research little recommendations are 
offered. This is why in the first part of this thesis, we take a specific interest in investigating the use of diagnostic instruments and the neurocognitive profile of high-average intelligent adolescents with ASD.

In the second part of this thesis, we were interested in gaining more insight in the mechanisms behind this disorder in high-functioning adolescents, as to our opinion this is an essential issue for understanding autism and for designing therapies and treatments. Nowadays it is generally recognized that besides the well-known triad of problems, many people with ASD experience problems in executive functioning (Hill, 2004). Given the central role of executive functions in both higher and lower cognitive processes, problems in these functions could provide a good explanation for the symptoms seen in ASD. As working memory is generally seen as a central process in many, if not all executive functions, we propose that it is highly plausible that working memory plays a more leading role in both the social and non-social symptoms seen in ASD. To date, however, studies have failed to give conclusive evidence about the relationship between working memory functioning and the symptoms seen in high-functioning adolescents with ASD. Therefore, in this thesis, we started to explore the role of working memory functioning in high-functioning adolescents' ASD symptomatology.

\section{Outline of this thesis}

It was this lack of diagnostic recommendations, the uncertainties about the neurocognitive profile and the still unknown aetiology in high-functioning adolescents with ASD, that inspired the theme of the current thesis. Alongside with Special Education School the Berkenschutse, we aimed to expand the current knowledge on the applicability of (standard) diagnostic instruments, the neurocognitive profile and the mechanisms behind ASD in (high) average intelligent adolescents. We were especially interested in the compensatory mechanisms these adolescents are often able to apply in more structured settings, and therefore, throughout this thesis, we placed an extra focus on the difference in behaviour in the laboratory setting and in real life situations. In the first section, we investigated the utility of the Achenbach instruments for autism screening (chapter 2). Standard diagnostic evaluation of adolescents for ASD is a time-consuming and expensive process and there is a need for a fast and economical screening instruments, able to identify adolescents at high risk of ASD and in need for in-depth evaluation from typically developing teens. The Achenbach instruments are often used broad general screening instruments, that have proven to be very valuable in screening for many psychological disorders in children between eight and eighteen years old, but until now, ASD is not one of them. In chapter $\mathbf{3}$, 
we were interested in the mentalizing and emotion recognition abilities in highfunctioning adolescents with ASD. We related our findings from the test setting with more spontaneous mentalizing skills in daily life. By doing so, we wanted to find out if the skills we measure during testing, actually relate to the skills these adolescents are able to use in real life. Chapter 4 aimed at getting more insight in the executive functioning abilities of these adolescent both in- and outside the structured laboratory setting. As high-functioning adolescents with ASD often show executive function deficits, we also wanted to investigate if executive function deficits were related to/ contribute to or even underlie ASD symptomatology.

In the second part of the thesis, the main focus lies on exploring the role of working memory functioning on ASD symptomatology in this high-functioning group adolescents, and on the possible use of compensatory mechanisms in structured settings. Therefore, in chapter $\mathbf{5}$ we reviewed the literature to investigate working memory functioning in high-functioning adolescents with ASD on a neurocognitive and neuroimaging level. To investigate this in our group of (high)average intelligent adolescents with ASD, we performed a functional MRI study (chapter 6). In this study we were interested in the network integrity of the brain networks involved in working memory functioning. We related our results with ASD symptomatology, to investigate a possible impact on cognitive and social functioning in our group of high-functioning adolescents. In chapter 7 we further explored the functional brain connectivity, by analysing the spatial connectivity and temporal neurodynamics of the resting-state networks. By looking at the spatial connectivity, we hoped to learn more about the spatial and temporal information processing in anatomical regions that show similar functional connectivity, and by investigating the temporal neurodynamics, we hoped to get more insight in the temporal causal effect dependencies between the different resting-state networks in high-functioning adolescents with ASD. As previous studies have suggested that alterations in excitatory/ inhibitory neurotransmitters might play a crucial role in ASD, we also examined glutamate and GABA neurotransmitter concentrations in our population by using $1 \mathrm{H}-\mathrm{MRS}$ (chapter 8 ).

Finally, chapter 9 is a general discussion of the results and conclusions from the constituting chapters. 



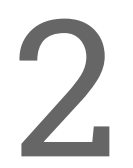

\section{USING THE ACHENBACH SCREENING INSTRUMENTS IN HIGH-FUNCTIONING ADOLESCENTS WITH AN AUTISM SPECTRUM DISORDER}

Submitted for publication as:

Barendse, E.M., Hendriks, M.P., Thoonen, G., Kessels, R.P., Aldenkamp, A.P. Using the Achenbach Screening Instruments in high-functioning adolescents with an autism spectrum disorder. 


\section{Abstract}

Background: Screening for autism spectrum disorders (ASDs) in high-functioning adolescents is complex; comorbid psychiatric disorders may exist that modulate or fully explain the expression of symptoms. Specific screening instruments for ASD lack the advantage of containing self-report and parent/teacher - report questionnaires and cannot screen for comorbid psychiatric disorders. Therefore, we investigated the utility of the Achenbach instruments for autism screening in adolescents.

Methods: Twenty-four high-functioning adolescents with ASD and twenty-four typically-developing controls, their parents and teachers were asked to fill in the required Achenbach questionnaire.

Results: Parent reports showed high scores on all Child Behavior Check List (CBCL) subscales in the ASD group, with an elevated pattern on the Withdrawn-depressed-, Social-, Thought-, Attention-, Internalizing- and Total-problems subscales. Sensitivity and specificity values on the CBCL were respectively moderate and high. Parent reports showed positive correlations with a standard autism diagnostic instrument. The high-functioning ASD adolescents scored significantly higher on almost all Youth Self Report (YSR) subscales, however effect sizes were small. The two groups could only be reliably discriminated by their amount of total problems on the YSR. Informant discrepancies were found between teachers and parents/ ASD adolescents.

Conclusion: Findings from the current study support the use of the CBCL as a screening tool for high-functioning ASD adolescents. The predictive value of the YSR as a screening tool for ASD adolescents was poor, which indicates that this instrument should not be used for this specific purpose although it does provide very important and necessary information for diagnostic and treatment purposes. 


\section{Introduction}

Autism Spectrum Disorder (ASD) is a heterogeneous neurodevelopmental disorder (DSM-IV), which can be subdivided into autistic disorder, Asperger syndrome, and pervasive developmental disorders not otherwise specified. Current diagnostic criteria cover a triad of clinical and behavioural signs that can be recognized throughout the spectrum, i.e. social impairments, communication difficulties and repetitive interests and behaviour. Individuals with ASD show a large heterogeneity in phenotype, severity, and type and frequency of symptoms. In addition, symptoms can change within one person over the course of development and other disorders, such as mental retardation, epilepsy, anxiety and mood disorders, frequently co-occur with ASD and may modify the threshold for presentation of symptoms (for overview see: Hill \& Frith, 2003). Finally, due to the shift from autism as a categorical disorder to a dimensional spectrum (Wing, 1996), more subtle features have been included in the definition of autism. Consequently prevalence rates increased being now 6 per 1000 children, with males being affected two to four times more often than females (Elsabbagh et al., 2012).

Especially high-functioning children often have these more subtle autism features, which could lead to missed diagnosis until secondary school when problems with learning and social interaction with peers begin to become apparent (Wing, 1997). Before that age, many high-functioning children with ASD are often able to compensate for their deficits. Some learn the 'rules' of social interaction by rote, although they lack the intuitive flexibility by which children with a normal development 'learn' and apply these social rules (Asperger, 1943; Hill \& Frith, 2003; Wing, 1997). Because of these compensation strategies, problems of high-functioning children with ASD are often difficult to measure in clinical or laboratory settings. In these structured settings, these children are able to successfully apply adaptive strategies (Rump et al., 2009; Salter et al., 2008). However, in more complex real-life (social) situations, they may often be unable to compensate for their deficits (Salter et al., 2008). Specifically during adolescence, social-cognitive skills become increasingly important, as they are essential in the process of becoming an independent adult. This illustrates the need to collect and use information about the adolescents' behaviour outside the laboratory or clinical setting (Asperger, 1943). Another complicating factor for diagnosing ASD in high-functioning adolescents is that comorbid psychiatric disorders may exist that modulate or fully explain the expression of symptoms (T Paus, Keshavan, \& Giedd, 2008). Specific screening instruments for ASD, such as the Social Communication Questionnaire, the Social Responsiveness Scale or the Autism-spectrum Quotient, miss the advantage of screening for these possible other or comorbid psychiatric disorders (S. Baron-Cohen, Hoekstra, 
Knickmeyer, \& Wheelwright, 2006; Chandler et al., 2007; Constantino et al., 2003). Consequently, as opposed to the disorder-specific screening instruments, broad screening methods are needed. Most frequently questionnaires are used for this purpose (Biederman et al., 2010). They have the advantage that the perception and qualification of parents, teachers and the adolescents about the adolescents' behaviour in real life can be assessed and used in the screening process (De Los Reyes, 2011).

Guidelines drawn up by the Dutch Association for Psychologist (Nederlands Instituut van Psychologen) state that when children show serious behavioural problems, the Achenbach screening instruments should be used. The Achenbach System of Empirically Based Assessment is a widely used and highly validated broader screening instrument for children and adolescents, containing the Child Behavior Checklist (CBCL), the Youth Self Report (YSR), and the Teacher Report Form (TRF) (Achenbach, 1996; Achenbach, 1997a, 1997b). According to the guidelines, if the score on the CBCL falls within a clinical range, further diagnostic evaluation should take place to investigate if other type of problems, such as Attention Deficit Hyperactivity Disorder or ASD, can explain the behavioural problems. The CBCL, YSR, and TRF for children between eight and eighteen years old have been designed to screen for the possibility of many psychological disorders, but until now, ASD is not one of them.

To the best of our knowledge, to date only three studies have investigated the CBCL as a screening tool for ASD in adolescents (Biederman et al., 2010; Mazefsky, Anderson, Conner, \& Minshew, 2011; So et al., 2012). These studies showed good discriminative ability between children with and without ASD on the CBCL and, that in addition to the CBCL, TRF subscales had a good predictive ability discriminating children with ASD from typically developing controls (So et al., 2012). Moreover, the predictive value increased when scores on the $\mathrm{CBCL}$ and TRF were combined. Although these results look promising, they are difficult to generalize to high-functioning adolescents with and without ASD. For example, all studies mentioned above included participants from a very wide age range, two studies included low functioning individuals (Mazefsky, Anderson, et al., 2011; So et al., 2012) and two included a significantly higher percentage females in the control group compared with the ASD group (Biederman et al., 2010; So et al., 2012), which is in accordance with known prevalence ratios but these gender differences may influence the results on their own (van der Ende \& Verhulst, 2005). To the best of our knowledge, no study investigated the discriminative ability of the YSR, whether or not in combination with the CBCL or TRF, for discriminating high-functioning adolescents with ASD from typically-developing controls. 


\section{Research aim}

Standard diagnostic evaluation of adolescents for ASD is a time-consuming and expensive process. It requires the participation of an expert who is trained in recognizing and evaluating behaviour related to ASD and comorbid disorders. This illustrates the need for a fast and economical screening instrument that would be able to identify adolescents at high risk of ASD and in need for an in-depth evaluation from typically developing teens. Validated, widely used broad questionnaires such as the Achenbach screening instruments could be used for this purpose. This would especially be useful, since the Dutch Association for Psychologist state that when children show serious behavioural problems, the Achenbach screening instruments should be used. The aim of the current case-control study is to test the capability of the CBCL and YSR to differentiate between a group of high-functioning adolescents with ASD and a control group of typically developing adolescents. We hypothesize, taken into account the results of earlier studies (Biederman et al., 2010; Mazefsky, Anderson, et al., 2011; So et al., 2012), that the CBCL and YSR will be able to discriminate the two groups. Here, we also look at possible discrepancies between parent- (CBCL), self- (YSR) and teacher-reports (TRF). Besides group effects, we also examine whether there is a relationship between these screening instruments and the Autism Diagnostic Observation Schedule (ADOS) (Lord, Rutter, DiLavore, \& Risi, 2010) a standard diagnostic measure of ASD. If the Achenbach Screening Instruments are a suitable screening tool for ASD, we expect to find a relationship between these questionnaires and the ADOS.

\section{Methods}

\section{Participants}

Twenty-nine high-functioning adolescents with ASD were recruited from a special secondary education school 'De Berkenschutse' located in Heeze, the Netherlands. Four of them, though diagnosed with a disorder on the autism spectrum, did not fulfil the ADOS diagnostic criteria and were excluded from the study. One participant did not return the questionnaires. Eventually, 24 adolescents with autism disorder $(n=3)$, Asperger syndrome $(n=10)$, multiple complex developmental disorder $(n=1)$, and pervasive developmental disorder not otherwise specified $(n=10)$ participated in this study. Participants with ASD fulfilled established diagnostic criteria according to the DSM-IV, as well as the autism algorithm cut-offs on the Autism Diagnostic Observation Schedule (ADOS) (Lord et al., 2010). The control group consisted of 24 typically developing adolescents and were recruited through advertisements in local newspapers. Participants of both groups were between 12-18 years old. Adolescents in the control group were excluded if they and/or one of their siblings and/or parent(s) 
had a diagnosis ASD. Further exclusion criteria for both groups were 1) a comorbid psychiatric disorder, 2) a significant hearing or visual impairment, 3) an inability to speak/understand the Dutch language and/or 4) a comorbid central neurologic or other somatic disorder.

All adolescents had an estimated FSIQ over 104 [range ASD: 107-129; range controls: 105-135], as measured with a short version of the Wechsler Intelligence Scale for Children (WISC-III) (Campbell, 1998; Donders, 1997, 2001). The two groups did not differ from each other with respect to age $(p=.42)$ and gender. There was a significant difference in Verbal Comprehension Index ( $\mathrm{VCl})(\mathrm{p}=.005)$, Perceptual Organization Index (POI) ( $p=.001)$ and FSIQ $(p=.001)$, with significantly higher estimated intelligence levels in the ASD group. However, effect sizes are small (Table 1). The study protocol was approved by the Medical Ethical Commission of the Maastricht University Medical Centre and written informed consent was obtained from all participants and their legal guardians.

Table 1 Demographic and descriptive data

\begin{tabular}{|c|c|c|c|c|}
\hline & C & ASD & & \\
\hline \multirow{2}{*}{$\begin{array}{l}\text { Gender } \\
\text { (male/female) }\end{array}$} & $22 / 2$ & $22 / 2$ & & \\
\hline & $M(s d)$ & $M(s d)$ & $\begin{array}{l}\text { Group } \\
\text { comparison }\end{array}$ & $n_{p}^{2}$ \\
\hline Age in months & $169.5(18.2)$ & $173.7(17.3)$ & $F(1,46)=.67, p=.416$ & .01 \\
\hline $\mathrm{VCl}$ & $114.6(9.2)$ & $122.5(9.2)$ & $F(1,46)=8.76, p=.005$ & .16 \\
\hline $\mathrm{POI}$ & $106.1(8.0)$ & $114.8(8.8)$ & $F(1,46)=12.72, p=.001$ & .22 \\
\hline FDI & $105.8(15.5)$ & $98.5(12.7)$ & $F(1,46)=3.15, p=.082$ & .06 \\
\hline FSIQ & $112.3(6.5)$ & $118.8(5.9)$ & $F(1,46)=13.55, p=.001$ & .23 \\
\hline
\end{tabular}

Note. Because we used a short version of the WISC-III, the Verbal Intelligence Quotient (VIQ) and the Performance Intelligence Quotient (PIQ) could not be calculated. $\mathrm{VCI}=$ Verbal Comprehension Index, $\mathrm{POI}=$ Perceptual Organization Index, FDI = Freedom from Distractibility Index, FSIQ = Full Scale Intelligence Quotient. $\mathrm{C}=$ Control group; $\mathrm{ASD}=\mathrm{ASD}$ group.

\section{Measures}

The Child Behavior Check List (CBCL)(Achenbach, 1996), Teachers Report Form (TRF) (Achenbach, 1997a) and Youth Self Report (YSR) (Achenbach, 1997b) are questionnaires assessing the adolescent's competence, behavioural problems, and emotional problems. All questionnaires have eight syndrome scales (Anxious/ 
depressed, Withdrawn/depressed, Somatic complaints, Social problems, Thought problems, Attention problems, Rule-breaking behavior and Aggressive behavior), and six DSM-oriented scales. Problems are further divided into Internalizing and Externalizing problems. The TRF has two additional subscales focusing on attention deficit/ hyperactivity problems: Inattention and Hyperactivity-impulsivity. Higher scores on the CBCL, YSR and TRF indicate more problems.

Autism Diagnostic Observation Schedule (ADOS) Module 4 (Lord et al., 2010) is a semi-structured, standardized assessment of social, communication, and language skills in adolescents and adults who are verbally fluent. During the assessment, activities and unstructured conversation are combined with structured situations and interview questions that offer a chance to observe a variety of behaviour. The goal of the ADOS module 4 is to determine how well the participant is able to initiate and sustain social interaction in situations with minimal structure and direction. The assessment takes 45-50 minutes, is recorded on video and scored afterwards by a trained examiner. The ADOS module 4 has four domain scores: 1) Communication, 2) Social interaction, 3) Communication-social interaction total and 4) Stereotyped behaviours and restricted interests. Higher scores on the domains indicate more problems on that specific domain.

\section{Statistical analysis}

Data analysis was performed using the Statistical Package for Social Sciences version 20.0. CBCL, YSR and TRF raw scores were used in all analyses, unless otherwise indicated. As there was a significant difference between the two groups on $\mathrm{VCI}, \mathrm{POI}$ and FSIQ, these variables could not act as a covariate in the statistical analysis (Field, 2009). However, as we did not find evidence in the literature for a meaningful relationship between normal- or above normal intelligence levels and behavioural problems as measured with the Achenbach instruments, we do not expect that these group differences in intelligence will influence the results.

In this study we adopted a cognitive-neuropsychiatric approach by investigating symptom dimensions (i.e. problem behaviour) rather than DSM classifications as such. Therefore, we used the eight syndrome scales and the Internalizing, Externalizing, and total problems scales of the CBCL, YSR and TRF in our analyses (Ebesutani et al., 2010). The raw scores of the four domains of the ADOS were used.

To test the ability of each of the CBCL and YSR syndrome scales to differentiate between the ASD group and the Control group, we conducted General Linear Model (GLM) analyses for each questionnaire, with group as independent variable and the various syndrome scales as dependent variables. A separate GLM was performed 
for the Internalizing, Externalizing, and Total problems scales of the CBCL and YSR. The descriptive data of the standard (t-scores) of the CBCL, YSR, and TRF scales were used to examine the percentage of adolescents at or above the borderline range for the syndrome scales (a t-score higher than or equal to 65) and Internalizing, Externalizing and Total problems scales (a t-score higher than 60 ) of the CBCL, YSR and TRF.

To estimate the number of participants required to perform Receiver Operating Characteristics (ROC) analyses, a sample size calculation was performed with MadCalc software. For alpha $=.05$, power $=.10$, and the predicted $A \cup C=.80$ (Ferdinand, 2008), results showed that a minimum of 17 participants were needed per group. As we included 24 participants per group, we can perform reliable ROC analyses. ROC analyses were performed on the t-scores of the various scales of the CBCL and the YSR. To measure the sensitivity and specificity of a scale, the standard cut-off criteria of the borderline cut-off scores of the syndrome scales $(t=65)$ and the Internalizing-, Externalizing-, and Total problems' scales $(\mathrm{t}=60)$ were used. Alternative cut-off points were considered based on the best balance between sensitivity and specificity levels. This best balance depends on the evaluation of the relative "cost" of false positives and false negatives. As the purpose of the current study is to distinguish possible cases of ASD from non-ASD cases, we choose for a cut-off with a low ratio of false positives (Ehlers, Gillberg, \& Wing, 1999). Positive likelihood ratios were also calculated.

Finally, to examine the relationship between the $C B C L$ and the $Y S R$, we performed correlational analyses for the whole group and for both groups separately. In the ASD group, one-tailed correlation analyses were performed to determine the relationship between the ADOS and the CBCL, YSR and TRF. We expected that a higher score on the ADOS would correlate positively with a higher score on the Achenbach questionnaires. To correct for the multiple comparisons only p-values less than 0.006 were interpreted as significant, according to the Bonferroni correction. Dependent t-tests between scores on the CBCL, YSR and TRF scales were explored to examine the differences between parent-, self-, and teacher ratings on the CBCL, YSR and TRF for both groups separately. 


\section{Results}

\section{CBCL}

The MANOVA revealed a significant main effect of group on the syndrome scales of the CBCL [Wilks $\wedge=.41, F(8 ; 39)=6.90, p<.001, \eta_{p}^{2}=.59$ ], and on the Internalizing, Externalizing, and Total problems scales of the CBCL [Wilks $\Lambda=.53, F(3 ; 44)=.53$, $p<.001, n_{p}^{2}=.47$ ], with high-functioning adolescents in the ASD group having higher scores on the CBCL than those in the Control group. Subsequent ANOVAs showed significant group effects on all measured CBCL scales (Table 2).

Of the ASD group, more than half of the adolescents scored on or above the borderline cut-off on the Withdrawn-depressed scale (62.5\%), Social problems scale (58.3\%), Thought problems scale (58.3\%), and the Internalizing and Total problems scales (both $62.5 \%$ ). Within the control group, almost all adolescents (varying from 91.6 to $95.8 \%$ ) scored below borderline on all scales (Table 2). Correlation analyses revealed significant positive correlations between the Social interaction scale of the ADOS and the Anxious-depressed problems $[r=.51 ; p=.005]$ and Internalizing problems $[r=.53 ; p=.004]$ subscales of the CBCL.

ROC analyses of the various syndrome scales of the CBCL as predictors of ASD showed fair to excellent predictive values with low sensitivity and high specificity values (Table 3).

\section{YSR}

A significant overall group effect (MANOVA) was found on the syndrome scales of the YSR [Wilks $\Lambda=.62, F(8 ; 39)=2.98, p=.011, \eta_{p}^{2}=.38$ ], and on the Internalizing, Externalizing, and Total problems scales of the YSR [Wilks $\Lambda=.78, F(3 ; 44)=.4 .20$, $\left.p=.011, n_{p}{ }^{2}=.22\right]$, with adolescents in the ASD group having higher scores on the YSR than those in the Control group. Subsequent ANOVAs showed significant group effects on all measured YSR scales, except the Anxious-depressed- and the Rule-breaking behavior scales [Anxious-depressed: $F(1,46)=3.76, p=.059, \eta_{p}^{2}=.08$; Rule-breaking behavior: $F(1,46)=3.14, p=.083, \eta_{p}^{2}=.06$ ] (Table 2).

Of the ASD group, 16.7 to 33.3 percent scored on or above borderline on the various YSR scales. Within the Control group, 4.2 to 8.4 percent of the adolescents scored on or above borderline level (Table 2). Correlation analyses revealed significant positive correlations between the Stereotyped behaviors and restricted interests subscale of the ADOS and the Social problems $[r=.56 ; p=.002]$ subscale of the YSR. $=O C$ analyses of the syndrome scales of the YSR as predictors of ASD showed mainly fair predictive values with low sensitivity values, and high specificity values (Table 3). 
Table 2 Group comparisons and clinical scores on the CBCL and YSR

\begin{tabular}{|c|c|c|c|c|c|c|}
\hline & C & ASD & & & C & ASD \\
\hline \multirow[t]{2}{*}{ Gender (male/female) } & $22 / 2$ & $22 / 2$ & & & $22 / 2$ & $22 / 2$ \\
\hline & $M(s d)$ & $M(s d)$ & $\begin{array}{l}\text { Group } \\
\text { comparison } \\
\text { (p) }\end{array}$ & $\eta_{p}^{2}$ & \multicolumn{2}{|c|}{$\begin{array}{l}\text { Above } \\
\text { borderline } \\
\text { (\%) }\end{array}$} \\
\hline \multicolumn{7}{|l|}{$\begin{array}{l}\text { CBCL } \\
\text { Syndrome scale }\end{array}$} \\
\hline Anxious-depressed & $1.79(2.40)$ & 7.29 (6.32) & $<0.001$ & .26 & 4.2 & 41.6 \\
\hline Withdrawn-depressed & $1.58(1.89)$ & 5.50 (3.39) & $<0.001$ & .35 & 4.2 & 62.5 \\
\hline Somatic problems & $0.71(0.96)$ & $3.50(3.41)$ & $<0.001$ & .25 & 0 & 25.0 \\
\hline Social problems & $1.21(2.41)$ & $6.46(3.73)$ & $<0.001$ & .42 & 4.2 & 58.3 \\
\hline Thought problems & $1.71(2.01)$ & $6.25(4.33)$ & $<0.001$ & .32 & 8.4 & 58.3 \\
\hline Attention problems & $2.46(2.43)$ & $7.75(3.44)$ & $<0.001$ & .45 & 0 & 16.7 \\
\hline Rule-breaking behavior & $0.92(1.86)$ & $2.33(1.90)$ & .012 & .13 & 0 & 0 \\
\hline Aggressive behavior & $2.33(3.29)$ & $7.63(6.35)$ & .001 & .22 & 0 & 20.8 \\
\hline Internalizing problems & $4.08(4.49)$ & $16.29(11.23)$ & $<0.001$ & .35 & 4.2 & 62.5 \\
\hline Externalizing problems & $3.25(4.90)$ & $9.96(7.26)$ & $<0.001$ & .23 & 8.4 & 25.0 \\
\hline Total problems & $14.21(15.65)$ & $51.13(25.98)$ & $<0.001$ & .44 & 4.2 & 62.5 \\
\hline \multicolumn{7}{|l|}{$\begin{array}{l}\text { YSR } \\
\text { Syndrome scale }\end{array}$} \\
\hline Anxious-depressed & $2.88(2.37)$ & $5.21(5.40)$ & .059 & .08 & 0 & 16.7 \\
\hline Withdrawn-depressed & $2.33(2.60)$ & $4.58(3.40)$ & .013 & .13 & 4.2 & 16.7 \\
\hline Somatic problems & $1.33(1.71)$ & $3.54(3.26)$ & .005 & .16 & 0 & 16.7 \\
\hline Social problems & $2.79(2.60)$ & $5.42(3.61)$ & .006 & .15 & 8.3 & 25.0 \\
\hline Thought problems & $1.88(1.39)$ & $4.96(4.46)$ & .002 & .19 & 0 & 20.9 \\
\hline Attention problems & $4.42(2.73)$ & $7.42(3.34)$ & .001 & .20 & 0 & 25.0 \\
\hline Rule-breaking behavior & $2.46(2.04)$ & $3.63(2.50)$ & .083 & .06 & 0 & 0 \\
\hline Aggressive behavior & $2.92(2.52)$ & $7.00(5.01)$ & .001 & .22 & 0 & 16.7 \\
\hline Internalizing problems & $6.54(5.29)$ & $13.33(10.83)$ & .008 & .14 & 8.4 & 33.3 \\
\hline Externalizing problems & $5.38(4.33)$ & $10.63(6.66)$ & .002 & .19 & 0 & 20.8 \\
\hline Total problems & 23.83 (13.98) & $45.38(26.30)$ & .001 & .21 & 0 & 29.2 \\
\hline
\end{tabular}


Table 3 Sensitivity/ specificity levels and likelihood ratios of the CBCL and YSR

\begin{tabular}{|c|c|c|c|c|c|c|c|c|}
\hline & 인 & 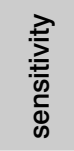 & $\begin{array}{l}\frac{2}{0} \\
\frac{0}{0} \\
\frac{0}{0} \\
\frac{0}{0}\end{array}$ & $\llbracket$ & 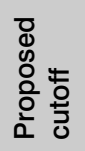 & 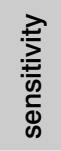 & 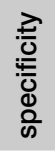 & $\llbracket$ \\
\hline \multicolumn{9}{|l|}{$\begin{array}{l}C B C L \\
\text { Syndrome scale }\end{array}$} \\
\hline Anxious-depressed & 0.79 & .42 & .96 & 9.9 & 65 & .42 & .96 & 9.9 \\
\hline Withdrawn-depressed & 0.81 & .49 & .96 & 10.9 & 64 & .63 & .96 & 14.9 \\
\hline Somatic problems & 0.83 & .25 & 1.00 & - & 56 & .63 & .83 & 6.5 \\
\hline Social problems & 0.90 & .58 & .96 & 13.9 & 63 & .63 & .96 & 14.9 \\
\hline Thought problems & 0.84 & .54 & .96 & 12.9 & 65 & .54 & .96 & 12.9 \\
\hline Attention problems & 0.89 & .17 & 1.00 & - & 60 & .50 & .96 & 11.9 \\
\hline Rule-breaking behavior & 0.74 & .00 & 1.00 & - & 53 & .46 & .92 & 5.5 \\
\hline Aggressive behavior & 0.81 & .21 & 1.00 & - & 63 & .25 & .96 & 5.9 \\
\hline Internalizing problems & 0.86 & .63 & .96 & 14.9 & 58 & .67 & .96 & 15.9 \\
\hline Externalizing problems & 0.82 & .25 & .92 & 3.0 & 52 & .67 & .88 & 5.3 \\
\hline Total problems & 0.92 & .63 & .96 & 14.9 & 60 & .63 & .96 & 14.9 \\
\hline \multicolumn{9}{|l|}{$\begin{array}{l}\text { YSR } \\
\text { Syndrome scale }\end{array}$} \\
\hline Anxious-depressed & 0.57 & .17 & 1.00 & - & 61 & .29 & .88 & 2.3 \\
\hline Withdrawn-depressed & 0.70 & .17 & .96 & 4.0 & 64 & .33 & .92 & 4.0 \\
\hline Somatic problems & 0.74 & .17 & 1.00 & - & 60 & .25 & .96 & 5.0 \\
\hline Social problems & 0.73 & .17 & .96 & 4.0 & 65 & .17 & .96 & 4.0 \\
\hline Thought problems & 0.73 & .21 & 1.00 & - & 57 & .38 & .96 & 8.9 \\
\hline Attention problems & 0.74 & .21 & 1.00 & - & 61 & .29 & .92 & 3.5 \\
\hline Rule-breaking behavior & 0.67 & 1.00 & 1.00 & - & 59 & .08 & .96 & 2.0 \\
\hline Aggressive behavior & 0.71 & .08 & 1.00 & - & 57 & .33 & .96 & 7.9 \\
\hline Internalizing problems & 0.71 & .33 & .92 & 4.0 & 62 & .29 & .96 & 7.0 \\
\hline Externalizing problems & 0.77 & .21 & 1.00 & - & 57 & .25 & .96 & 6.0 \\
\hline Total problems & 0.81 & .29 & 1.00 & - & 59 & .29 & .96 & 7.0 \\
\hline
\end{tabular}

Note: $\mathrm{AUC}=$ Area Under the Curve; $\mathrm{LR}=$ Likelihood Ratio. 


\section{TRF}

Of the ASD group, the percentages of participants scoring on or above borderline scores on the various subscales of the TRF were as follows: Anxious-depressed problems $25 \%$, Withdrawn-depressed problems 18.8\%, Somatic problems 0\%, Social problems $31.3 \%$, Thought problems 31.3\%, Attention problems 12.5\%, Rule breaking behavior $6.3 \%$, Aggressive behavior $12.5 \%$, Internalizing problems $56.3 \%$, Externalizing problems $31.3 \%$, Total problems $50 \%$. Correlation analyses revealed no significant correlations between the domain scores of the ADOS and subscales of the TRF.

\section{CBCL vs YSR vs TRF}

To determine if there are discrepancies between parent- (CBCL), self- (YSR) and teacher-reports (TRF), correlational analyses were performed. Whole-group analyses showed significant positive correlations, with medium to large effect sizes between all syndrome scales and the Internalizing-, Externalizing-, and Total problems scales of the CBCL and YSR. In the Control group, significant positive correlations were

Table 4 Control group comparisons between the CBCL and YSR

\begin{tabular}{|c|c|c|c|c|c|}
\hline \multirow{4}{*}{ Gender (male/female) } & \multirow{2}{*}{\multicolumn{2}{|c|}{$\begin{array}{c}C \\
22 / 2\end{array}$}} & \multirow{4}{*}{ 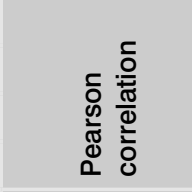 } & \multirow{4}{*}{ 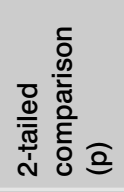 } & \multirow{4}{*}{$\begin{array}{l}\frac{0}{N} \\
\frac{N}{0} \\
\frac{\mathscr{T}}{0}\end{array}$} \\
\hline & & & & & \\
\hline & \multirow{2}{*}{$\begin{array}{l}\text { CBCL } \\
\text { M(sd) }\end{array}$} & \multirow{2}{*}{$\begin{array}{c}\text { YSR } \\
\text { M (sd) }\end{array}$} & & & \\
\hline & & & & & \\
\hline \multicolumn{6}{|l|}{ Syndrome scale } \\
\hline Anxious-depressed & $1.79(2.40)$ & $2.88(2.37)$ & $r=.64 p<.001$ & .015 & .48 \\
\hline Withdrawn-depressed & $1.58(1.89)$ & $2.33(2.60)$ & $r=.62 p=.001$ & ns & .35 \\
\hline Somatic problems & $0.71(0.96)$ & $1.33(1.71)$ & ns & ns & .38 \\
\hline Social problems & $1.21(2.41)$ & $2.79(2.60)$ & ns & .015 & .48 \\
\hline Thought problems & $1.71(2.01)$ & $1.88(1.39)$ & ns & ns & .09 \\
\hline Attention problems & $2.46(2.43)$ & $4.42(2.73)$ & ns & .005 & .54 \\
\hline Rule-breaking behavior & $0.92(1.86)$ & $2.46(2.04)$ & $r=.62 p=.001$ & $<.001$ & .68 \\
\hline Aggressive behavior & $2.33(3.29)$ & $2.92(2.52)$ & $r=.64 p<.001$ & ns & .23 \\
\hline Internalizing problems & $4.08(4.49)$ & $6.54(5.29)$ & $r=.58 p=.001$ & .014 & .49 \\
\hline $\begin{array}{l}\text { Externalizing } \\
\text { problems }\end{array}$ & $3.25(4.90)$ & $5.38(4.33)$ & $r=.66 p<.001$ & .013 & .49 \\
\hline Total problems & $14.21(15.65)$ & 23.83 (13.98) & $r=.52 p=.004$ & .004 & .56 \\
\hline
\end{tabular}


found between all CBCL and YSR scales, except for the Somatic problems, Social problems, Thought problems and Attention problems scales, which were not significantly correlated (Table 4). In the ASD group, a significant positive correlation was present between the CBCL and the YSR on the Anxious-depressed, Somatic problem and Internalizing problems scale (Table 5). Between the CBCL and TRF, in the ASD group, a significant positive correlation was found on the Anxious-depressed scale (Table 6). The YSR and TRF showed significant positive correlations on the Anxious-depressed-, Social problems-, Internalizing problems-, and Total problems scale (Table 7).

Table 5 ASD group comparisons between the CBCL and YSR

\begin{tabular}{|c|c|c|c|c|c|}
\hline \multirow{4}{*}{ Gender (male/female) } & \multicolumn{2}{|c|}{ ASD } & \multirow{4}{*}{ 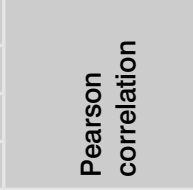 } & \multirow{4}{*}{ 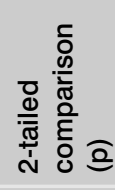 } & \multirow{4}{*}{$\begin{array}{l}\frac{0}{N} \\
\frac{1}{0} \\
\frac{\Phi}{0} \\
\frac{\Phi}{0}\end{array}$} \\
\hline & \multicolumn{2}{|c|}{$22 / 2$} & & & \\
\hline & CBCL & YSR & & & \\
\hline & $M(s d)$ & $M(s d)$ & & & \\
\hline \multicolumn{6}{|l|}{ Syndrome scale } \\
\hline Anxious-depressed & $7.29(6.32)$ & $5.21(5.40)$ & $r=.61 p=.001$ & ns & .38 \\
\hline Withdrawn-depressed & $5.50(3.39)$ & $4.58(3.40)$ & ns & ns & .24 \\
\hline Somatic problems & $3.50(3.41)$ & $3.54(3.26)$ & $r=.76 p<.001$ & ns & .02 \\
\hline Social problems & $6.46(3.73)$ & $5.42(3.61)$ & ns & ns & .22 \\
\hline Thought problems & $6.25(4.33)$ & $4.96(4.46)$ & ns & ns & .26 \\
\hline Attention problems & $7.75(3.44)$ & $7.42(3.34)$ & ns & ns & .09 \\
\hline Rule-breaking behavior & $2.33(1.90)$ & $3.63(2.50)$ & ns & .044 & .41 \\
\hline Aggressive behavior & $7.63(6.35)$ & $7.00(5.01)$ & ns & ns & .08 \\
\hline Internalizing problems & $16.29(11.23)$ & $13.33(10.83)$ & $r=.64 p<.001$ & ns & .31 \\
\hline Externalizing problems & $9.96(7.26)$ & $10.63(6.66)$ & ns & ns & .07 \\
\hline Total problems & $51.13(25.98)$ & $45.38(26.30)$ & ns & ns & .20 \\
\hline
\end{tabular}

Note: $\mathrm{ASD}=\mathrm{ASD}$ group. Because of the multiple comparisons, only $\mathrm{p}$-values less than 0.03 were interpreted as significant.

Dependent t-tests between scores on the CBCL and YSR in the Control group showed significant differences on almost all scales, except on the Withdrawn-depressed-, Somatic-, Thought problems-, and Aggressive behavior scales. Mean scores on all the YSR scales were higher than the equivalent mean scores on the CBCL in this group (Table 4). In the ASD group, dependent t-tests between the scores on the CBCL and the YSR showed a significant difference on the Rule breaking behavior scale. Most mean subscale scores were higher on the CBCL than on the YSR (Table 5). 
Table 6 ASD group comparisons between the CBCL and TRF

\begin{tabular}{|c|c|c|c|c|c|}
\hline \multirow{4}{*}{ Gender (male/female) } & \multirow{2}{*}{\multicolumn{2}{|c|}{$\begin{array}{l}\text { ASD } \\
15 / 1\end{array}$}} & \multirow{4}{*}{ 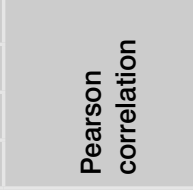 } & \multirow{4}{*}{ 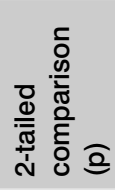 } & \multirow{4}{*}{$\begin{array}{l}\frac{D}{N} \\
\frac{N}{0} \\
\frac{0}{0} \\
\frac{\mathbb{T}}{0}\end{array}$} \\
\hline & & & & & \\
\hline & \multirow{2}{*}{$\begin{array}{l}\text { CBCL } \\
M(s d)\end{array}$} & \multirow{2}{*}{$\begin{array}{c}\text { TRF } \\
\text { M (sd) }\end{array}$} & & & \\
\hline & & & & & \\
\hline \multicolumn{6}{|l|}{ Syndrome scale } \\
\hline Anxious-depressed & $7.94(6.82)$ & $5.88(6.39)$ & $r=.63 p=.005$ & ns & .35 \\
\hline Withdrawn-depressed & $6.00(3.18)$ & $4.63(3.78)$ & ns & ns & .33 \\
\hline Somatic problems & $3.81(3.76)$ & $0.75(1.00)$ & ns & .002 & 69 \\
\hline Social problems & $7.06(3.11)$ & $4.75(4.22)$ & ns & .043 & .50 \\
\hline Thought problems & $6.56(4.07)$ & $3.19(2.90)$ & ns & .005 & .65 \\
\hline Attention problems & $8.44(3.50)$ & $18.13(9.46)$ & ns & .002 & .70 \\
\hline Rule-breaking behavior & $2.06(1.81)$ & $2.25(3.07)$ & ns & ns & .07 \\
\hline Aggressive behavior & $7.696 .89)$ & $5.63(5.34)$ & ns & ns & .25 \\
\hline Internalizing problems & $17.75(11.75)$ & $11.25(8.15)$ & ns & .019 & .56 \\
\hline Externalizing problems & $9.75(7.52)$ & $7.88(7.62)$ & ns & ns & .19 \\
\hline Total problems & $54.19(23.27)$ & $47.94(26.01)$ & ns & ns & .19 \\
\hline
\end{tabular}

Note: $\mathrm{ASD}=$ ASD group. 16 TRF questionnaires were returned, therefore $\mathrm{n}=16$. Because of the multiple comparisons, only $\mathrm{p}$-values less than 0.03 were interpreted as significant.

Dependent t-tests between the CBCL and the TRF in the ASD group showed significant differences between the Somatic problems-, Social problems-, Thought problems-, Attention problems-, and the Internalizing problems scales. Of these, only the mean score on the Attention problems subscale was higher on the TRF (Table 6). The dependent t-tests between the YSR and TRF in the ASD group showed significant differences between the Somatic problems-, and Attention problems scales, whereby the adolescents reported more somatic problems than their teachers, and the teachers reported more attention problems than the adolescents (Table 7). 
Table 7 ASD group comparisons between the YSR and TRF

\begin{tabular}{|c|c|c|c|c|c|}
\hline \multirow{4}{*}{ Gender (male/female) } & \multicolumn{2}{|c|}{ ASD } & \multirow{4}{*}{ 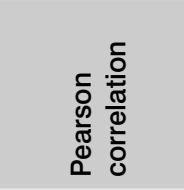 } & \multirow{4}{*}{ 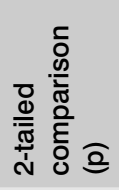 } & \multirow{4}{*}{$\begin{array}{l}\frac{D}{N} \\
\frac{N}{0} \\
\frac{\Phi}{0} \\
\frac{4}{0}\end{array}$} \\
\hline & \multicolumn{2}{|c|}{$15 / 1$} & & & \\
\hline & YSR & TRF & & & \\
\hline & $M(s d)$ & $M(s d)$ & & & \\
\hline \multicolumn{6}{|l|}{ Syndrome scale } \\
\hline Anxious-depressed & $5.94(5.69)$ & $5.88(6.39)$ & $r=.85 p<.001$ & ns & .02 \\
\hline Withdrawn-depressed & $4.63(3.59)$ & $4.63(3.78)$ & ns & ns & .00 \\
\hline Somatic problems & $3.81(3.19)$ & $0.75(1.00)$ & ns & $<.001$ & .76 \\
\hline Social problems & $5.943 .82)$ & $4.75(4.22)$ & $r=.64 p=.004$ & ns & .34 \\
\hline Thought problems & $5.38(4.80)$ & $3.19(2.90)$ & ns & ns & .46 \\
\hline Attention problems & $7.81(3.47)$ & $18.13(9.46)$ & ns & $<.001$ & .79 \\
\hline Rule-breaking behavior & $2.81(2.20)$ & $2.25(3.07)$ & ns & ns & .17 \\
\hline Aggressive behavior & $6.81(5.10)$ & $5.63(5.34)$ & ns & ns & .24 \\
\hline Internalizing problems & $14.38(11.47)$ & $11.25(8.15)$ & $r=.77 p<.001$ & ns & .40 \\
\hline Externalizing problems & $9.63(6.53)$ & $7.88(7.62)$ & ns & ns & .25 \\
\hline Total problems & $46.81(27.85)$ & $47.94(26.01)$ & $r=.65 p=.003$ & ns & .05 \\
\hline
\end{tabular}

Note: $A S D=$ ASD group. 16 TRF questionnaires were returned, therefore $n=16$. Because of the multiple comparisons, only p-values less than 0.03 were interpreted as significant.

\section{Discussion}

The main purpose of the current study was to evaluate the ability of the CBCL and YSR to discriminate a group of high-functioning adolescents with a disorder on the autism spectrum from a group of typically developing teens. We further looked at the relationship between the CBCL/ YSR/ TRF and the ADOS, and at discrepancies between parent-, self-, and teacher-reports.

Our results show that the $\mathrm{CBCL}$ is indeed able to discriminate high-functioning adolescents with ASD from teens with a typical developmental trajectory. However, results should be interpreted cautiously. Adolescents with ASD scored significantly higher than the controls on all CBCL subscales. This effect is particularly noteworthy in the Withdrawn-depressed-, Social-, Thought-, Attention-, Internalizing- and Total problems subscales. The Attention problems subscale aside, sensitivity and specificity values on these subscales were respectively moderate (ranging from 
49-63\%) and high (96-100\%) using the standard test cut-off scores. Clinical scores were also highest on these subscales; more than half of the adolescents with ASD scored on or above the borderline cut-off on the Withdrawn-depressed scale $(62.5$ $\%)$, Social problems scale (58.3\%), Thought problems scale (58.3\%), and Internalizing and Total problems scales (both $62.5 \%$ ). These subscales clearly overlap with diagnostic classification criteria of ASD, with items such as "withdrawn, does not interact with others", "there is little that (s)he likes", "does not get along with other boys or girls", "awkward movements or clumsy", "strange behavior", "strange ideas", "repeats certain behavior", and "speech problems" (under which parents often rank communication difficulties).

Our findings are consistent with earlier research that also showed an elevated pattern on the CBCL Withdrawn-, Social-, Thought and Total problems scales in children with ASD. Based on this pattern of elevations, typically developing children and children with ASD can be distinguished with a high level of sensitivity and specificity (Biederman et al., 2010; Mazefsky, Anderson, et al., 2011; Sikora, Hall, Hartley, Gerrard-Morris, \& Cagle, 2008). In spite of these clear results, this specific ASD pattern on the $\mathrm{CBCL}$ appears difficult to translate to clinical practice, as on an individual level, only ten adolescents with ASD exhibited this specific elevation pattern. Furthermore, all of these ten adolescents showed elevations on or above borderline on the Anxious-depressed-, Attention problems-, and Aggressive behavior scales. Thus, on a group-level a clear ASD pattern exists, but this pattern is not always present on an individual level. This discrepancy may well be a reflection of the large heterogeneity of the disorder, which makes it difficult to translate group findings to individuals, and the specific ASD pattern on the CBCL in individuals may be expected in larger samples. The present findings stress the notion that the $\mathrm{CBCL}$ can be used as a screening instrument for ASD, especially considering the high probability that these adolescents might have other (comorbid) problems that also need psychiatric evaluation (So et al., 2012). However, the CBCL should and cannot be used as the tool to finalize a diagnosis.

In contrast to the CBCL, the YSR does not seem to be a reliable screening tool for ASD. Although high-functioning adolescents with ASD scored significantly higher on almost all YSR subscales (except the Anxious-depressed and Rule-breaking behavior), effect sizes were small. Moreover, the two groups could only be reliably discriminated by their amount of total problems on the YSR. Exploratory research showed no indication of a specific elevation pattern, as earlier found on the CBCL. Likewise, previous studies did not report this pattern of elevations on the YSR and TRF in high-functioning adolescents with ASD (Barnhill et al., 2000; De Los Reyes, 2011; S. A. Johnson, Filliter, \& Murphy, 2009; Lerner, Calhoun, Mikami, \& De Los 
Reyes, 2012; Nicpon et al., 2010). The use of self-report screening questionnaires has often been investigated in typically developing adolescents and although discrepancies between self- and parent-reports are often found (as discussed in the next section), self-reports of adolescents are generally seen as reliable and are essential to obtain adequate diagnostic information (Ebesutani, Bernstein, Martinez, Chorpita, \& Weisz, 2011; Mazefsky, Kao, \& Oswald, 2011). In youth with ASD, there is some more discussion on the reliability of self-reports. Some studies report that high functioning adolescents with ASD may be able to report their own psychiatric symptoms but only to a certain degree. Possibly because they find it difficult to adequately identify and report their own symptoms, consistent with earlier reports of difficulties in the cognitive domain of alexithymia in adults with ASD (Berthoz \& Hill, 2005; Mazefsky, Kao, et al., 2011). However, other studies have found that although children with ASD may not have adequate insight into their own behaviour until a later developmental stage, high functioning adolescents with ASD are able to provide such information reliably (S. Baron-Cohen et al., 2006; Kuusikko et al., 2008; Sheldrick, Neger, Shipman, \& Perrin, 2012). Thus, although the YSR does not seem to be a good screening tool for identifying ASD in high-functioning adolescents, it remains an important tool to gather information about the adolescents' perception of their behavioural and adaptive skill functioning (Barnhill et al., 2000; Nicpon et al., 2010).

In the second part of the study, we examined informant discrepancies between high-functioning adolescents with ASD, their parents, and their teachers. In the current study, adolescents in the control group tended to report more problems than their parents, while the high-functioning adolescents with ASD showed considerable agreement with both their parents and their teachers when rating their behaviour. Informant discrepancies were found between parent reports and teacher reports in the ASD group; compared with parents, teachers generally report fewer and less significant concerns. These results are in line with earlier studies that showed considerable agreement between parents and high-functioning adolescents with ASD in reporting psychiatric symptoms (Hurtig et al., 2009) and reported clinically relevant discrepancies between parent and teacher reports (Barnhill et al., 2000; Koning \& Magill-Evans, 2001) with teachers reporting significantly fewer concerns about the adolescents compared with parents.

Informant discrepancies are a robust finding throughout various studies and are thought to reflect variations across settings in the expression of an individual's behaviour (De Los Reyes, 2011). These discrepancies are also thought to reflect differences in perception between informants, differences in opportunities to observe problem behaviour in various settings, and differences in social comparisons and biases due to expectations (Barnhill et al., 2000; Nicpon et al., 2010). Furthermore, 
such discrepancies may be explained by the developmental stage of adolescence; adolescents spend more time with their peers and share less time and information with their parents. Consequently, a lot of behaviour and feelings of the adolescent may go unnoticed by parents (Steinberg \& Morris, 2001; Verhulst \& van der Ende, 1992). This could, for example, explain the differences in opinion between parents and adolescents in the control group.

In the past, these informant discrepancies were considered as measurement errors and were often neglected. Nowadays, the prevailing view is that these discrepancies between informants might yield knowledge of the development of child psychopathology that cannot be explained by the individual informants' reports. As the aetiology of ASD is still unknown and its diagnosis still has to be made on basis of behavioural symptoms, it is necessary to document the behavioural characteristics specific to particular contexts as precisely as possible, versus characteristics that are not context-specific (Achenbach, 2011), especially since there is no definite way to determine who is an 'accurate' informant; the parents, adolescents or the teachers (De Los Reyes, 2011).

In comparison with the ADOS, our results show positive relationships between the adolescents' ability to initiate and maintain social interactions (as measured with the ADOS) and the anxious-depressed- and internalizing problems as reported by parents. In addition, on the CBCL positive relationships were found between the amount of stereotyped behaviours and restricted interests, as observed with the ADOS, and the amount of social problems that the adolescents reported themselves. On the YSR, symptoms of mood and anxiety problems are often present in children and adolescents with ASD (Hill \& Frith, 2003) and are associated with higher levels of stereotyped behaviour and more social reciprocity problems (Sukhodolsky et al., 2008). Some researchers even speculated that, although children with ASD had a good understanding of how to act among peers, symptoms of anxiety and stress undermined their actual use of social skills in natural settings (Donnellan, Leary, \& Patterson-Robledo, 2006). From the adolescents' perspective, one can imagine that stereotyped behaviours and restricted interests could have a negative impact on social interactions with peers. In contrast with the results from both the parents and the adolescents, problems as reported by the teachers were not associated with scores on the ADOS. In the current study, these discrepancies can best be explained by two factors; 1) all adolescents in the current study visit a school for special secondary education that is highly specialized in teaching adolescents with ASD. This school is able to create a highly structured educational context to their students, which may prevent possible problem behaviour at school. And 2) almost all teachers of this school exclusively teach adolescents with ASD. Through their daily work, they 
may be used to (a certain degree of) problem behaviour. This may influence their perception and evaluation of the adolescents' behaviour.

A certain limitation of this study is that all adolescents with ASD that participated have an average or above average intelligence. Although these adolescents represent a significant part of all high-functioning adolescents with ASD, results may not be representative for all high-functioning adolescents with ASD. Comorbid psychiatric diagnoses in the ASD group were an exclusion criterion and parents were explicitly asked if their child had other psychiatric diagnoses apart from ASD. Additional limitations are that TRF and ADOS scores of the control group were missing. In future studies it would be interesting to compare adolescents with ASD and a control group on these measures as well. Furthermore, although our sample size calculation showed that our sample of 24 participants per group was profound enough, it would also be interesting to see if a larger sample will enhance the power of the Achenbach instruments to screen for ASD symptomatology.

Overall, findings from the current study provide support for the use of the CBCL as a screening tool for high-functioning adolescents with ASD. Especially the elevation pattern of Withdrawn-depressed-, Social-, Thought-, Internalizing-, and Total problems on the CBCL indicates the need for in-depth evaluation for ASD, in agreement with previous studies. The predictive value of the YSR as a screening tool for adolescents with ASD, however, was poor, which indicates that this instrument should not be used for this specific purpose although it does provide very important and necessary information for diagnostic and treatment purposes. Informant discrepancies were found between teachers and parents / adolescents with ASD. Although these differences were often ignored in the past, they will give clinicians more insight in the variations of the adolescents' behaviour across settings, and about the differences between the informants' perception and evaluation of this behaviour. This makes obtaining information from adolescents and their parents/ teachers indispensable in the screening process. Other highly validated but more specific screening instruments for ASD, such as the Social Communication Questionnaire, the Social Responsiveness Scale or the Autism-spectrum Quotient (S. Baron-Cohen et al., 2006; Chandler et al., 2007; Constantino et al., 2003), lack this advantage of containing self-report and parent/ teacher - report questionnaires. This makes it impossible to make a good distinction between context-free and context-specific behavioural characteristic of the adolescents, and does not acknowledge possible differences in perception of this behaviour between the adolescent and his/ her parent/teacher. Furthermore, as these instruments are specifically designed to screen for ASD, they do not have the advantage of screening for other or comorbid psychiatric disorders that may modulate or fully explain the expression of ASD 
symptoms. This makes the often used and validated Achenbach screening instruments, with their broad survey across various behavioural scales and self-, parent-, and teacher-ratings ideal for screening adolescents suspected of having ASD and/ or other or comorbid disorders. Finally, it is important to highlight that no behaviour checklist should be used as the only standardized measure of autistic symptoms in diagnosing ASDs. 



\section{SOCIAL BEHAVIOUR AND SOCIAL COGNITION IN HIGH-FUNCTIONING ADOLESCENTS WITH AN AUTISM SPECTRUM DISORDER (ASD): TWO SIDES OF THE SAME COIN?}

Submitted for publication as:

Barendse, E.M., Hendriks, M.P., Thoonen, G., Kessels, R.P., Aldenkamp, A.P. Social Behaviour and Social Cognition in high-functioning adolescents with an Autism Spectrum Disorder (ASD): two sides of the same coin? 


\section{Abstract}

Background: Of the triad of symptoms found in Autism Spectrum Disorder (ASD), i.e. social impairments, communication difficulties and repetitive interests and behaviour, the social impairments are found the most stable and common throughout the lifespan. These social impairments typically manifest themselves in abnormalities in reciprocal interactions and difficulties in the expression and recognition of emotions. Although peer interactions become especially important during adolescence, little is known about the mentalizing abilities of high-functioning adolescents with ASD.

Methods: Here, we compared the mentalizing skills and emotion recognition abilities of 21 high-functioning adolescents with ASD and 21 matched controls. All adolescents had estimated above average verbal intelligence levels. Spontaneous social abilities and task related social abilities were measured using questionnaires, tasks and the Autism Diagnostic Observation Schedule.

Results: The results confirm social impairment in daily life situations in adolescents with ASD, but were not found during task related performance.

Conclusion: The use of more explicit cognitive or verbally mediating reasoning techniques and a lesser tendency of high-functioning adolescents with ASD to search for and use social information in more natural environments are further discussed. 


\section{Introduction}

Autism Spectrum Disorder (ASD) is a heterogeneous neurodevelopmental syndrome, which is characterized by persistent deficits in social communication and social interaction across multiple contexts and restricted, repetitive patterns of behavior, interest or activities (DSM 5). Individuals with ASD show a large heterogeneity in phenotype, severity, and type and frequency of symptoms. In addition, symptoms can change within one person over the course of development (Hill \& Frith, 2003; Levy, Mandell, \& Schultz, 2009; Wing, 1997). However, as initially stated by Kanner (1943), and still relevant today: "The outstanding, 'pathognomonic', fundamental disorder is the children's inability to relate themselves in the ordinary way to people and situations from the beginning of life" (p.242). This statement is supported by recent research showing that of the triad of symptoms, social impairments are the most stable symptom not only in children, but also in adolescents, adults and elderly and across all developmental levels (Shattuck et al., 2007). As such, they are considered more central and persistent than the other core symptoms of autism (James, Mukaetova-Ladinska, Reichelt, Briel, \& Scully, 2006; Seltzer, Shattuck, Abbeduto, \& Greenberg, 2004; Shattuck et al., 2007). These social impairments typically manifest themselves in abnormalities in reciprocal interactions and difficulties in the expression and recognition of emotions (Bauminger, 2002).

Although the social difficulties are independent of age and developmental level, their impact may disproportionally increase during adolescence (Nicpon et al., 2010; E. Pellicano, 2010b). Compared with children, adolescents are more sociable and form more complex relationship with their peers. This forming of social networks contributes to their identity development and affect their self-esteem. Adolescents are also more sensitive to acceptance and rejection by their peers (Blakemore, 2008; Steinberg \& Morris, 2001). That is, during this developmental period, opinions and evaluations of peers become increasingly salient and many adolescents with ASD begin to notice how they differ from their peers (Burnett, Bird, Moll, Frith, \& Blakemore, 2009; Crone \& Dahl, 2012; Steinberg, 2005). This is especially noticeable in high-functioning adolescents with ASD as they, more than their low-functioning counterparts, seek and initiate social interaction with peers (Bauminger et al., 2003; Hauck et al., 1995). The effects hereof may be found in the development of anxiety or mood disorders and feelings of social loneliness (the perceived lack of social involvement with peers) in high-functioning adolescents with ASD (Bauminger, 2002; White, Oswald, Ollendick, \& Scahill, 2009; White \& Roberson-Nay, 2009). Thus, despite the general assumption of social aloofness in ASD, these reports of loneliness and the relatively high degree to which high-functioning adolescents with ASD initiate social interactions with peers (Bauminger et al., 2003), may indicate that they do want to take part in 
more satisfying social relationships. Despite the different impact social impairments have in the different developmental stages, numerous studies have been performed on the underlying mechanisms of social impairments in younger children with ASD or in adolescents with ASD with low to average intelligence levels, only limited research has been done on social cognitive function in high-functioning adolescents with ASD with high-average to above-average intellectual functioning.

For social interaction to be successful, it is necessary to understand another persons' emotions, intentions, believes and knowledge (N. David et al., 2010). This information is needed to predict another person's behavior and adjust one's own behavior accordingly. The ability to impute and understand the mental states of others, and recognize that these states may differ from your own is called 'mentalizing' or having a Theory of Mind (ToM) (S Baron-Cohen et al., 1985; Premack \& Woodruff, 1978; Vollm et al., 2006). The ability to mentalize depends on a range of both lower level mechanisms, such as face and emotion processing, gaze direction and the detection of animacy, as well as higher executive function mechanisms such as attention and working memory (see review: Stone \& Gerrans, 2006). Of these, particularly the human face and its emotional expressions plays an important role in mentalizing, as it constitutes an important source of information about a person's inner state. Developmental trajectories of both mentalizing and emotion recognition abilities reach near adult levels around age eleven, with a further refinement of these skills during adolescence and across the adult lifespan (S Baron-Cohen et al., 1985; for review see: Brune \& Brune-Cohrs, 2006; Perner \& Wimmer, 1985; Rump et al., 2009).

Although mentalizing problems in individuals with ASD have been a major topic in autism research since the early 80 s, there is still little information available on the mentalizing abilities in high-functioning adolescents with ASD. To the best of our knowledge only two studies investigated these abilities in a group of high-functioning adolescents with either Asperger syndrome or autism (Kaland, Callesen, MollerNielsen, Mortensen, \& Smith, 2008; Kaland, Smith, \& Mortensen, 2007). Participants in these studies were 21 early-to-late adolescents with ASD and 20 typically developing matched controls. In their first study Kaland and colleagues (Kaland et al., 2007) used an adjusted and contextually more complex version of the Strange Stories Test (F. G. Happe, 1994a), whereby the participants had to make mental-state inferences versus physical state inferences in a story context. Adolescents with ASD made more errors compared with controls and had slower reaction times especially on the mental-state interference task. The authors concluded that the adolescents with ASD had more problems compared with controls in making inferences about mental states. The second study was seemingly conducted with the same participant group (Kaland et al., 2008). In this study the performance of the participants on three 
different advanced theory of mind tasks (i.e. The Eyes task (S. Baron-Cohen, Wheelwright, Hill, Raste, \& Plumb, 2001), the Strange Stories Test (F. G. Happe, 1994a), and the Stories of Everyday Life (Kaland et al., 2002) were compared. The results showed that the adolescents with ASD performed worse than the typically developing controls on all three mentalizing tasks. In both studies, the authors made no direct link between the performance of the adolescents with ASD on the mentalizing tasks and their more spontaneous mentalizing abilities as for example seen on the Autism Diagnostic Observation Schedule (ADOS) a standard diagnostic measure of ASD (Lord et al., 2010, psychometric properties are provided in the method section), or between the tasks and the adolescents' social functioning outside the laboratory setting.

Also important for social interaction in daily life is the ability to perceive and label expressions. However, emotion perception has also only been marginally investigated in high functioning adolescents with ASD (Rump et al., 2009; Tracy, Robins, Schriber, \& Solomon, 2011) and the results contradict each other. Some studies showed that high-functioning adolescents with ASD were able to recognize (complex) emotional facial expressions just as fast and accurate as typically developing teens (C. R. Jones et al., 2011; Rump et al., 2009; Tracy et al., 2011), whereas other studies showed that they were less accurate than their typically developing counterparts (Brosnan, Johnson, Grawmeyer, Chapman, \& Benton, 2015; Greimel et al., 2014; Kuusikko et al., 2009; Mazza et al., 2014; M. J. L. Smith, Montagne, Perrett, Gill, \& Gallagher, 2010; Wallace et al., 2011). Only the result of one study (Wallace et al., 2011) were related to the behavioral problems these adolescents with ASD have in everyday life. Wallace and colleagues found that diminished perceptual sensitivity to sad faces was positively related to the number of social communication symptoms (as measured with the ADOS) and adaptive functioning (as measured with the adaptive behavior assessment system-II) in a group of 42 high functioning $(I Q>80)$ adolescents with ASD when compared with a normal control group.

In the current study we address these shortcomings by examining both mentalizing and emotion recognition abilities of high-functioning adolescents with ASD and matched typically developing controls. We aimed to relate their results with more spontaneous mentalizing skills as seen on the ADOS and their social functioning as measured with self-reports about friendship and feelings of acceptance. By doing so, we want to get more insight in the mentalizing abilities of these high-functioning teens with ASD and find out if the skills we measure in the laboratory setting relate to the skills they are able to use in real life. Based on the existing literature, we assume to find impaired mentalizing abilities in high-functioning adolescents with ASD compared to the control group. Due to the scarcity and contradictory evidence of previous reports, emotion recognition abilities and the relation between the adolescents' 
social abilities in- and outside the structured setting of the laboratory were assessed in an exploratory way.

\section{Methods}

\section{Participants}

Twenty-nine high-functioning adolescents with ASD were recruited from a special secondary education school 'De Berkenschutse' located in Heeze, the Netherlands. Of this group, twenty-five adolescents fulfilled the established diagnostic criteria according to the DSM-IV as well as the autism algorithm cut-offs on the ADOS. Controls were twenty-nine adolescents without a history of psychological or psychiatric illness. Adolescents of both groups followed pre-university education or senior general secondary education. The groups were matched on education level, years of education, age and gender. Exclusion criteria for both groups were: an estimated verbal intelligence below 110, a diagnosis for a psychological disorder or psychiatric disease as formulated in the DSM-IV, such as attention-deficit and disruptive behaviour disorders, separation anxiety disorders, selective mutism, reactive attachment disorder of infancy or early childhood, anxiety disorders and mood disorders; appearance of additional variables that can influence cognitive functioning such as pathology of the Central Nervous System, and a significant visual or hearing impairment. Four adolescents with ASD and eight controls did not have an estimated above-average verbal intelligence and were excluded. Eventually 21 adolescents ( 1 female) with autism disorder $(n=3)$, Asperger syndrome $(n=9)$, and pervasive developmental disorder not otherwise specified $(n=9)$, and 21 typically developing control adolescents (4 females) participated in this study.

All adolescents had an estimated verbal intelligence over 109 [range ASD: 114-144; range controls: 111-144], as measured with a short version of the Wechsler Intelligence Scale for Children (WISC-III) (Campbell, 1998; Donders, 1997, 2001). Because we used a short version of the WISC-III, the Verbal Intelligence Quotient (VIQ) could not be calculated. However, we could estimate the Verbal Comprehension Index (VCl). The two groups did not differ from each other with respect to education level, years of education, age, and gender. Although the groups were matched on education level and years of education, there was a significant difference in estimated verbal intelligence and full scale intelligence levels, however, effect sizes are small (Table 1). The study protocol is approved by the Medical Ethical Commission of the Maastricht University Medical Center. In accordance with the declaration of Helsinki, informed consents were obtained from all adolescents and their parents or caregivers. 
Table 1 Demographic and descriptive data

\begin{tabular}{|c|c|c|c|c|}
\hline & Control & ASD & & \\
\hline & $M(s d)$ & $M(s d)$ & $\begin{array}{l}\text { Group } \\
\text { comparison }\end{array}$ & $\eta_{p}^{2}$ \\
\hline Gender (male/female) & $17 / 4$ & $20 / 1$ & $\begin{array}{l}U=189.00, z=-1.412 \\
p=0.343\end{array}$ & \\
\hline Age in months & $170.8(18.9)$ & $173.0(18.2)$ & $\begin{array}{l}F(1,40)=0.592 \\
p=0.446\end{array}$ & 0.015 \\
\hline $\mathrm{VCl}$ & $118.5(7.7)$ & $123.6(7.4)$ & $\begin{array}{l}F(1,40)=4.728 \\
p=0.036\end{array}$ & 0.106 \\
\hline $\mathrm{POI}$ & $105.5(8.1)$ & $114.5(9.2)$ & $\begin{array}{l}F(1,40)=11.413 \\
p=.002, \eta^{2}=.222\end{array}$ & .222 \\
\hline FDI & $103.9(14.0)$ & $98.9(11.7)$ & $\begin{array}{l}F(1,40)=1.570, n s \\
(p=.218), n^{2}=.038\end{array}$ & .038 \\
\hline FSIQ & $113.0(7.4)$ & $119.0(5.5)$ & $\begin{array}{l}F(1,40)=8.894 \\
p=0.005\end{array}$ & 0.182 \\
\hline \multicolumn{5}{|l|}{ ADOS } \\
\hline Communication & - & $3.95(1.60)$ & - & - \\
\hline $\mathrm{RSI}$ & - & $7.10(2.51)$ & - & - \\
\hline SBRI & - & $1.00(1.05)$ & - & - \\
\hline
\end{tabular}

Note: Because we used a short version of the WISC-III, the Verbal Intelligence Quotient (VIQ) could not be calculated. $\mathrm{VCI}=$ Verbal Comprehension Index, FSIQ = Full Scale Intelligence Quotient. Control= Control group, ASD $=$ ASD group, RSI = Reciprocal Social Interaction, SBRI = Stereotyped Behaviors and Restricted Interests * senior general secondary education/pre-university education.

\section{Materials and Procedure}

All participants who returned the informed consent form (adolescent and their parents/ caregivers) were asked to fill out and return questionnaires with items related to their spontaneous social functioning. The ADOS module 4 was administered to all adolescents with ASD to confirm the diagnosis and to get an impression of their more spontaneous social functioning skills as well. All participants further participated in a broad neuropsychological assessment. In the current study, we discuss the results of the social cognition tasks: the Director task and Emotion Recognition task.

\section{Social functioning.}

The Child Behavior Check List and Youth Self Report (Achenbach, 1996; Achenbach, 1997b) are questionnaires assessing the adolescent's competence, behavioral problems, and emotional problems. Of these questionnaires, raw scores of the items 
"Number of friends", "Frequency of contact with friends", "Behavior with others" and "Behavior alone" of the Social subscale were used. Both the construct validity and criterion validity of the Child Behavior Check List and Youth Self Report are sufficient (A. Evers, Vliet-Mulder,J.C. van, \& Groot, C.J., 2007).

The Self-Perception profile for Adolescents is a questionnaire that inquires the adolescents self-concept on six domains of competence and assesses a global sense of self-worth (Treffers, 2002). The Self-Perception profile for Adolescents has a good internal consistency, test-retest reliability, and construct validity (A. Evers, VlietMulder,J.C. van, \& Groot, C.J., 2007; Treffers, 2002). In the current study, the raw scores of the subscales "Social acceptance" and "Close friendships" were used.

The Autism Diagnostic Observation Schedule (ADOS) (Lord et al., 2010) module 4 is a semi-structured, standardized assessment of social, communication, and language skills in adolescents and adults who are verbally fluent. The ADOS has an excellent interrater reliability and internal consistency, and the sensitivity and specificity for ASD relative to non-spectrum disorders are also found to be excellent (Lord et al., 2000). During the ADOS assessment, activities and unstructured conversation are combined with structured situations and interview questions that offer a chance to observe a variety of behavior. The goal of the ADOS module 4 is to determine how well the participant is able to initiate and sustain social interaction in situations with minimal structure and direction. The assessment takes 45-50 minutes, is recorded on video and scored afterwards by a trained examiner. The ADOS module 4 has four domain scores: 1) Communication, 2) Social interaction, 3) Communication-social interaction total and 4) Stereotyped behaviors and restricted interests. Higher scores on the domains indicate more problems on that specific domain.

\section{Social cognition: Theory of Mind.}

The Director task is a computerized perspective taking ToM task, which requires the participant to infer the perspective and intentions of another person (the director) and to act upon it in order to select the appropriate response (Apperly et al., 2010). This is an experimental task, which in previous research showed good discriminative validity (Dumontheil, Apperly, \& Blakemore, 2010; Symeonidou, Dumontheil, Chow, \& Breheny, 2016). For this study we translated this task into Dutch (for an example of the English version see: Dumontheil et al., 2010).

Participants view stimuli in a cabinet with $4 \times 4$ shelves containing eight different objects. Five slots were occluded at the rear side of the cabinet. In each trial, the participant hears a director giving an instruction to move one of the eight objects in a particular direction. Next, the participant is asked to click on the referred object and 
drag it to the appropriate slot in the cabinet. In the Director condition, a 'director' is standing behind the cabinet, from whose perspective the contents of five slots are not visible (Fig.1). In this condition, participants have to take into account the perspective of the director when executing the instructions. Participants were told that objects in slots with a gray background were visible only to them, whereas the other objects could be seen from either side of the cabinet.

\section{Your View}

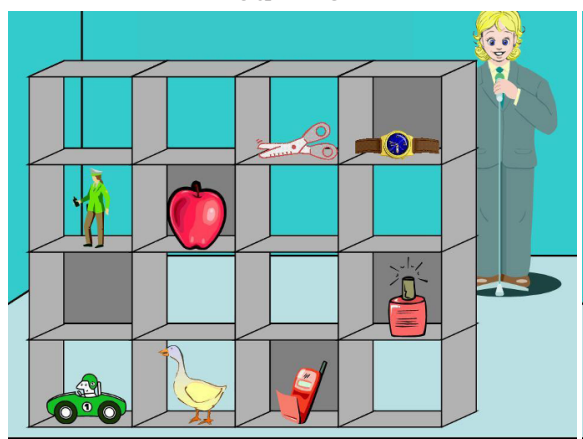

Director's View

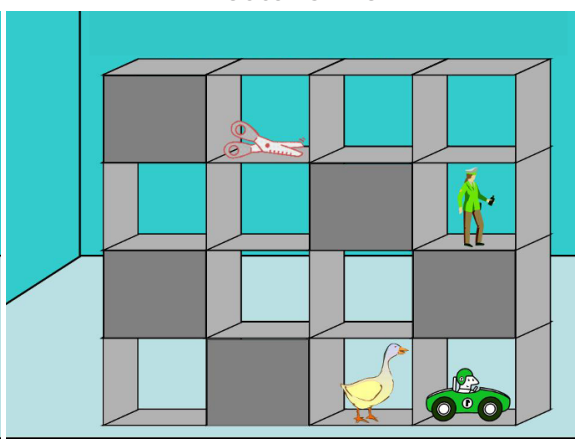

Figure 1 Instruction screens to explain the task to participants. The cabinet on the left is shown from the participants point of view, and the cabinet on the right is shown from the Director's point of view.

There are three kinds of instructions: 1) In Experimental trials, the instruction referred to one object ('target') given from the director's point of view but would refer to another object ('distractor') if one assumed participants' perspective. As such, participants need to take the director's perspective into account to give a correct response in the Experimental trials (Fig. 2A). 2) In Control trials, the arrangement of the objects on the shelves was identical to the Experimental trails except that an irrelevant object replaces the distractor object (e.g. the instruction referred to an object that is visible to both participant and the director; Fig. 2B). 3) Filler trial instructions refer only to objects in empty slots, that is, to objects that are visible to both the director and participant (e.g. the camera in Fig. 2). In the No-Director condition, no director was standing behind the cabinet and participants are told that the instructions only refer to objects in the clear slots; objects in slots with a grey background should be ignored (Fig. $2 \mathrm{C}$ and D). Other than this, the No-Director trails were identical to the Director trials and had Experimental, Control and Filler trials. 


\section{Director condition}

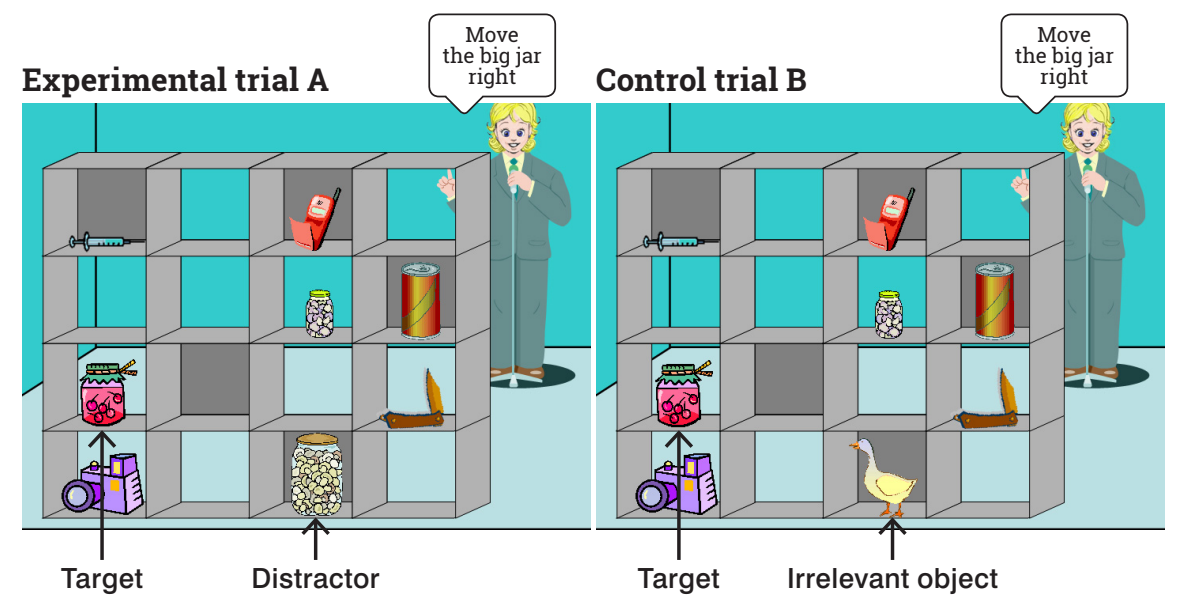

\section{No-Director condition}

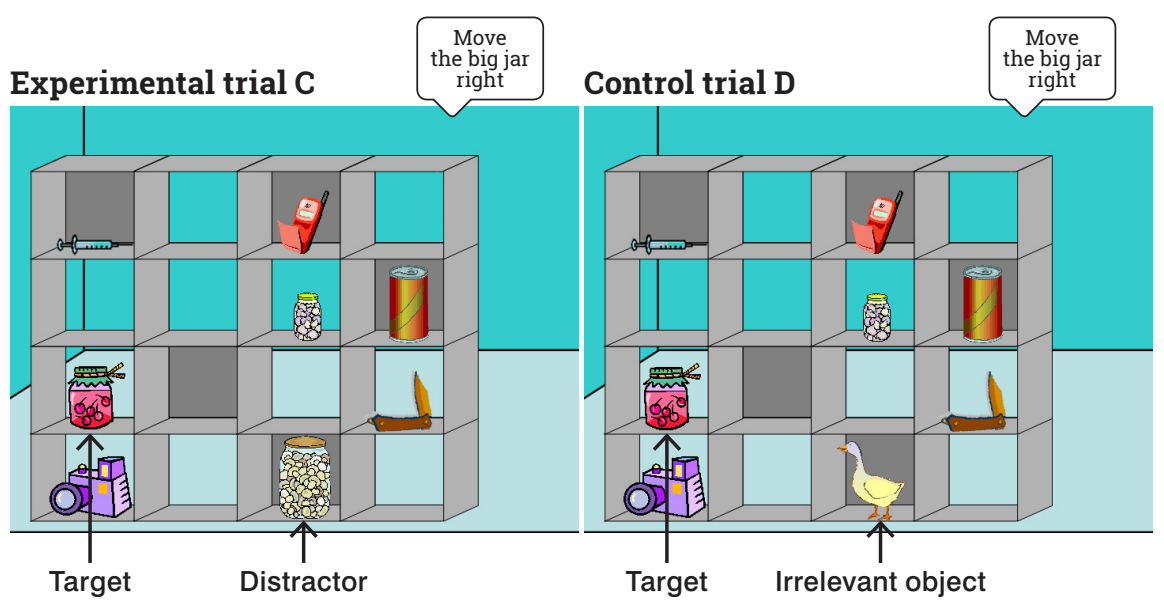

Figure 2 Stimuli of the Director task. (A and B) Director condition; The participant hears the instruction "move the big jar right' from the Director. In an Experimental trial (A), if the participant does not take the director's perspective into account, he or she would move the jar with stones instead of the jar with cherries, which cannot be seen from the directors side. In a control trial (B), the distractor is replaced by an irrelevant object (goose). (C and D) No-Director condition; The participant is told that instructions do not refer to items in slots with a gray background, therefore, the correct responses are the same as in de Director. 
The test consists of two sets (one for each condition) of eight different shelf-object configurations. Each stimulus was presented for 2 seconds before the first auditory instruction was given. Three auditory instructions of each 2.2 seconds were given per stimulus. After the instructions, participants had 3.6 seconds to make their response. In total there were eight Control trials, eight Experimental trials and 48 filler trials in each condition (Director and No-Director). The Director condition was assessed before the No-Director condition in all participants, in order to prevent participants to apply the strategy of the No-Director condition in the Director condition.

\section{Social cognition: Emotion recognition.}

The ERT is an Emotion Recognition Task in which dynamically morphed facial expressions are presented at different levels of intensities (Montagne, Kessels, De Haan, \& Perrett, 2007). This is an experimental task, which in previous research showed good discriminative validity (M. J. L. Smith et al., 2010). Participants view short video clips which show a neutral face gradually changing into one of the six basic facial emotions; anger, disgust, fear, happy, sadness and surprise (Ekman, 1992; Ekman \& Friesen, 1971). After viewing the video clip, the participant has to choose from a list of six emotions, which one was shown on the video. No time restriction was used. The version of the ERT used here, included morphs from neutral to four different intensities; from $0-40 \%, 0-60 \%, 0-80 \%$ and $0-100 \%$. The video clips were presented in a fixed order of 4 blocks containing twenty-four trials, always starting with the lower intensities after which the intensity was increased.

\section{Statistical Analysis}

Data analysis was performed using the Statistical Package for Social Sciences version 20.0. Raw scores were used in all analyses, unless otherwise indicated. There was a significant difference between the two groups on $\mathrm{VCl}$ and $\mathrm{FSIQ}$, therefore these variable could not act as a covariate in the statistical analysis (Field, 2009; G. A. Miller \& Chapman, 2001). Group comparisons on social behavior, ToM and emotion recognition abilities were made conducting MANOVAs and mixed model MANOVAs. Correlation analyses were conducted to investigate the relationship between ToM (Director task) and emotion recognition (ERT) abilities, and between ToM / emotion recognition abilities and spontaneous social functioning (ADOS and questionnaires) in both groups separately. 


\section{Results}

\section{Social functioning}

A MANOVA on the Child Behavior Checklist revealed significant differences on the 'Number of friends' $\left[p<0.001, \eta_{p}^{2}=0.35\right]$, the 'Frequency of contact with friends' $\left[p=0.011, \eta_{p}^{2}=0.15\right]$, and the 'Behavior (getting along) with others' $[p<0.001$, $\left.\eta_{p}^{2}=0.36\right]$ subscales on the Child Behavior Checklist. Parents of adolescents with ASD reported that their children had fewer real friends, had less contact with their friends outside school, and were less able to get along with others compared with the reports of the parents of adolescents in the Control group. On the Youth Self Report, a significant difference was found on the 'Behavior (getting along) with others' $\left[p=0.001, \eta_{p}^{2}=0.23\right]$ subscale; adolescents with ASD reported that they were less able to get along with others compared with the reports of the Control group (Table 2). On the Self-Perception Profile for Adolescents, adolescents with ASD report no significant differences on the subscales 'Social acceptation' [Control: $m=15.33$, $s d=2.78$; ASD: $m=13.90, s d=3.13$ ] and 'Close friendships' [Control: $m=17.14$, $s d=2.87$; ASD $m=15.67, s d=3.72]$, when compared with Controls.

Table 2 Social behavior as measured with the CBCL and YSR

\begin{tabular}{|l|c|c|c|c|}
\hline & $\mathbf{C}$ & ASD & & \\
\hline Gender (male/female) & $\mathbf{1 7 / 4}$ & $\mathbf{2 0 / 1}$ & & \\
\hline & $\mathbf{M}(\mathbf{s d})$ & $\mathbf{M}(\mathbf{s d})$ & $\mathbf{p}$ & $\mathbf{n}_{\mathbf{p}}$ \\
\hline $\mathbf{C B C L}$ & & & & \\
\hline Number of real friends & $2.4(0.67)$ & $1.2(0.98)$ & $<0.001$ & 0.345 \\
\hline Seeing friends outside school & $1.0(0.78)$ & $0.4(0.60)$ & 0.011 & 0.152 \\
\hline Getting along with others & $1.4(0.43)$ & $0.8(0.44)$ & $<0.001$ & 0.362 \\
\hline Plays/ works alone & $1.2(0.51)$ & $1.5(0.75)$ & 0.157 & 0.049 \\
\hline YSR & & & & \\
\hline Number of real friends & $2.6(0.50)$ & $2.3(0.73)$ & 0.109 & 0.064 \\
\hline Seeing friends outside school & $1.1(0.85)$ & $0.8(0.77)$ & 0.185 & 0.045 \\
\hline Getting along with others & $1.3(0.36)$ & $1.0(0.31)$ & 0.001 & 0.230 \\
\hline Plays/ works alone & $1.4(0.51)$ & $0.4(0.75)$ & 0.694 & 0.004 \\
\hline
\end{tabular}

Note: $\mathrm{CBCL}=$ Child Behavior Checklist, $\mathrm{YSR}=$ Youth Self Report, Control = Control group, $\mathrm{ASD}=\mathrm{ASD}$ group. 


\section{Social cognition: Theory of Mind}

A mixed model MANOVA with condition (Director, No-director) and Trial type (Control, Experimental) as within-subject factors and Group (Control, ASD) as between-group factor revealed highly significant main effects for Condition $[F(1,36)=16.750, p<0.000$, $\left.\eta_{p}^{2}=0.32\right]$, and Trial type $\left[F(1,36)=29.366, p<0.000, \eta_{p}^{2}=0.45\right]$, with more errors being made on the Director task vs. the NoDirector task, and more errors being made on the experimental trails than on the control trials. No overall effect of Group was found $\left[F(1,36)=0.097, p=0.757, \eta_{p}^{2}=0.003\right.$ ] (see Fig. 3). Although not significant, a trend in interaction was found between Condition and Trial type $[F(1,36)=3.918$, $\left.p=0.055, n_{p}^{2}=0.10\right]$, with more errors being made on the experimental trials in the Director condition vs. the No-Director condition. All other interaction effects were non-significant with F-values below one.

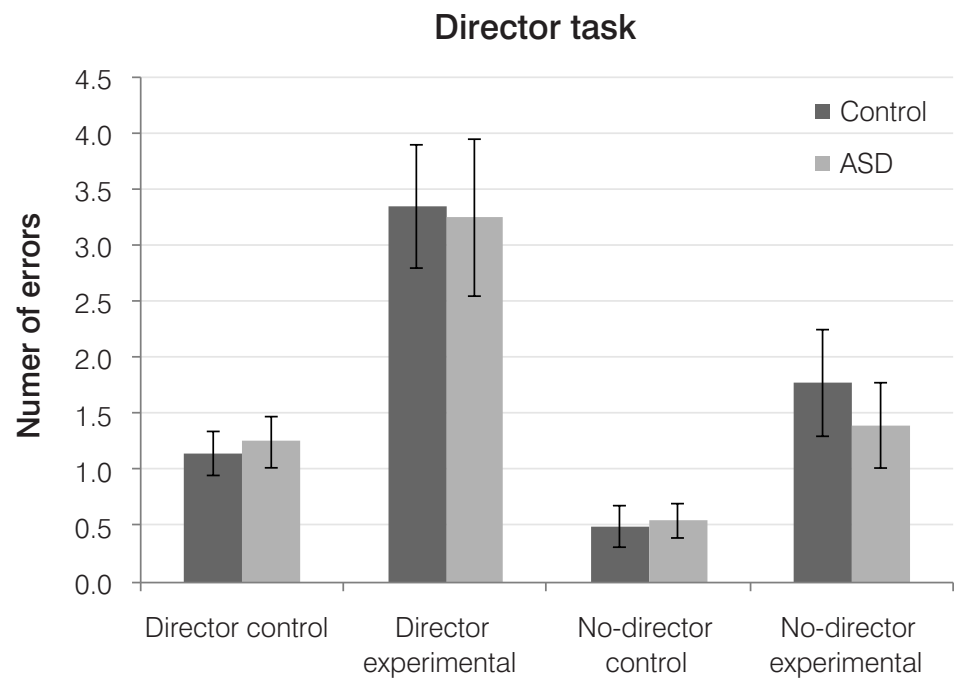

Figure 3 Mean percentage of errors and standard errors on the Director task for adolescents with ASD and Controls.

\section{Social cognition: Emotion recognition}

A mixed model MANOVA with Emotion (Anger, Disgust, Fear, Happy, Sad, Surprise) and Intensity (40\%, 60\%, 80\%, 100\%) as within-subject factors and Group (Control, ASD) as between-group factor showed significant main effects for Emotion $\left[F(5,36)=158.608, p<0.000, \eta_{p}^{2}=0.96\right]$, and Intensity $[F(3,38)=14.799, p<0.000$, $\left.\eta_{p}{ }^{2}=0.54\right]$. The effect of Intensity showed a linear trend $[F(1,40)=36.082, p<0.000$, 


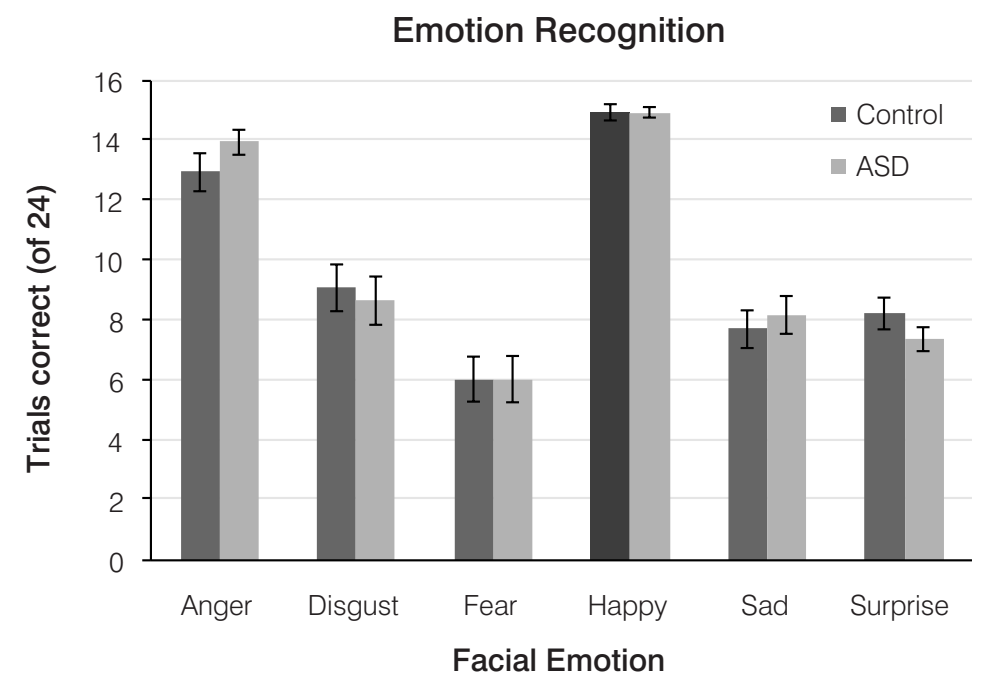

Figure 4 Mean performance and standard error for the six basic (ERT) emotions in adolescents with ASD and Controls.

$\left.\eta_{p}^{2}=0.47\right]$, the higher the intensity, the higher the scores. No overall Group effect was found $\left[F(1,40)=0.200, p=0.657, n_{p}^{2}=0.01\right]$ (see Fig. 4). There was a significant interaction effect between Emotion and Intensity $\left[F(15,26)=6.285, p=<0.000, n_{p}^{2}=0.78\right]$ (see Fig. 5). No other significant interaction effects were found (all $F$-values $<1$ ). In both groups, $80 \%$ of the participants scored above the $2^{\text {nd }}$ percentile on the various Emotions compared with the standard scores of the ERT.

\section{Correlation}

Correlation analyses showed no significant correlation between intelligence ( $\mathrm{VCl}$, FSIQ) and the tasks (Director task, ERT). Correlation analysis between the two tasks showed a significant correlation between the ERT and the 'Director task Experimental items' in the Control group [ $r=-0.547, p=0.013]$. In the ASD group no significant correlations between these two tasks were found.

Correlation analysis between the two tasks and more spontaneous social functioning (as measured with the ADOS and items of the Child Behavior Checklist, Youth Self Report and Self-Perception Profile for Adolescents) showed no significant correlations in both groups.

In all analyses, no differences were found when in- or excluding outliers. 
Sad

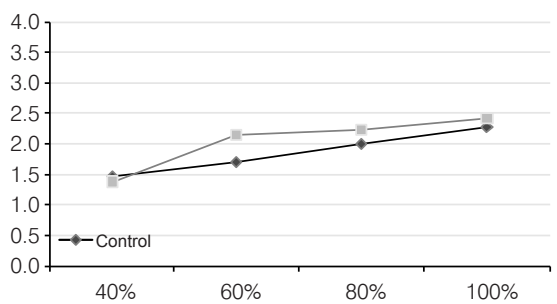

Disgust

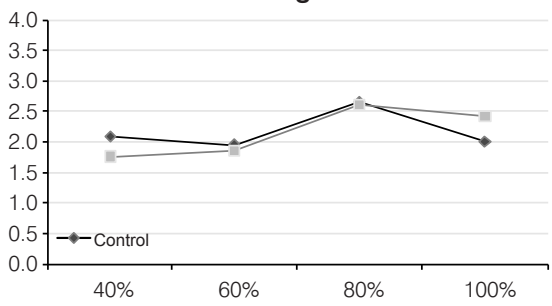

Fear

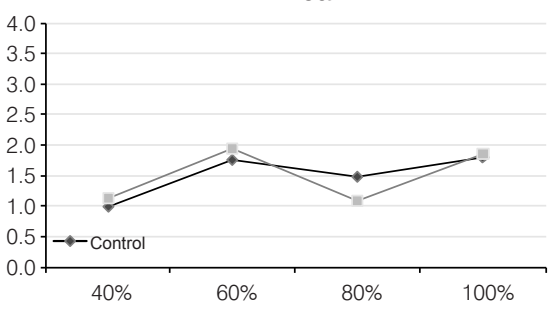

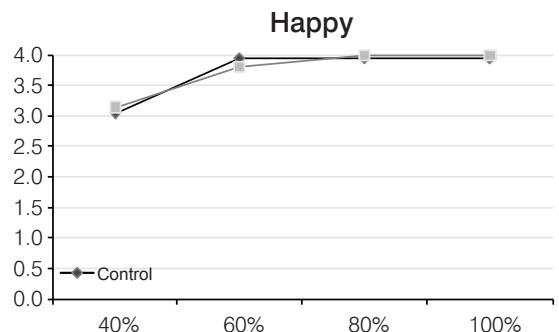

Anger

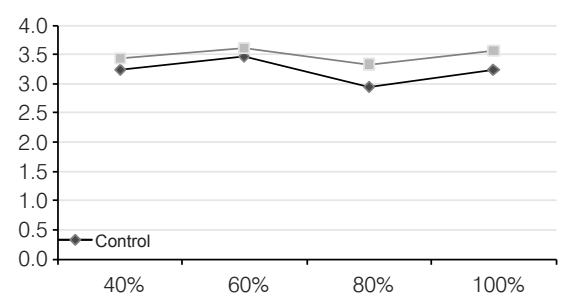

Surprise

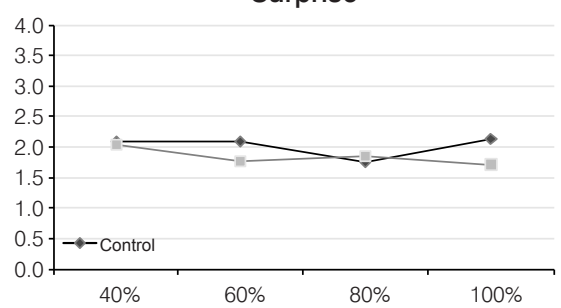

Figure 5 Mean performance for the six basic emotions ( $y$-axis; max=4) presented at different intensity levels (x-axis; 40-100\%) for adolescents with ASD and Controls.

\section{Discussion}

The first aim in this study was to compare mentalizing and emotion recognition abilities in high-functioning adolescents with ASD and a matched normal developing control group. We wanted to compare the groups on both their spontaneous social abilities and their task-related social abilities. Our results confirm the expected social impairments in high-functioning adolescents with ASD in daily life situations, however, we could not confirm these impairments during task performance. 
In line with other studies, friendship characteristics of high-functioning adolescents with ASD differ from those of their normal developing peers (Bauminger, Solomon, Aviezer, Heung, Brown, et al., 2008; Bauminger, Solomon, Aviezer, Heung, Gazit, et al., 2008; Kuo, Orsmond, Cohn, \& Coster, 2013). According to their parents, the adolescents with ASD in our study have fewer real friends and they meet their friends less often outside school compared with the control group. In general they report that their children are less able in getting along with others. Considering the three dimensions of friendship in adolescence (i.e. companionship, intimacy-trust, closenessaffection), and the fact that individuals with ASD by definition have peer difficulties (Bauminger, Solomon, Aviezer, Heung, Gazit, et al., 2008; Mazurek \& Kanne, 2010), we expected to find these results.

This vision of friendship characteristics as reported by their parents, however, is not in concordance with how the adolescents with ASD describe these friendships themselves. Although they do report being less able in getting along with others, their reports on social acceptance, the number of close friendships, and the frequency of contact with their friends were comparable with controls. These results may be explained by differences in the definition of friendship that parents and high-functioning adolescents with ASD use. For example, Kuo and colleagues (2013) suggested that adolescents with ASD may consider peers who have similar interests to be a friend, whereas their parents may consider someone a friend only then if there is a relationship with mutual interactions and emotional interchanges. Another explanation may be that parents underestimate the adolescents' friendships because they assume that because their child has ASD, (s)he per definition would have fewer (closer) friends (Kuo et al., 2013). Considering that the adolescents with ASD in our study went to a special education school, their parents may also have less insight in the quality of the friendships, as out-of-school contact with friends mainly occurs via social media, or because adolescents in general may be inclined to a lesser extent to share their social life with their parents. However, scores on the item 'Insight in social relationships' from the ADOS, show that more than $70 \%$ of the adolescents with ASD in our study have no or limited insight into the nature of social relationships and/or his/her own role in it. In combination with the fact that all adolescents with ASD in our study scored on- or above the ASD cut-off level on 'Reciprocal social interaction problems' of the ADOS, it is more likely that these adolescents with ASD use a different definition of friendship. Despite these differences in friendship definition of high functioning adolescents with ASD and the problems that they have in their everyday life with forming and maintaining social relationships (Scheeren, de Rosnay, Koot, \& Begeer, 2013), these problems were not confirmed on task level. Even on an advanced ToM test (Director task) and on the recognition of more complex emotions such as 'surprise' of the ERT, where the participant has to make inferences about someone else's thoughts 
(Loveland et al., 1997), we do not find significant differences between adolescents with ASD and the normal control group.

Although most often not investigated in high-functioning adolescents with ASD, this paradox of social interaction problems in everyday life while they show no deficits on neuropsychological social tests, have been reported in ASD literature repeatedly (Dahlgren \& Trillingsgaard, 1996; C. R. Jones et al., 2011; Kessels, 2010; Rump et al., 2009; Russell \& Hill, 2001; Scheeren et al., 2013; Senju, Southgate, White, \& Frith, 2009; Tracy et al., 2011). This apparent discrepancy may be explained by the use of compensation techniques that high-functioning individuals with ASD are often able to apply in more structured settings. By using explicit cognitive or verbally mediated reasoning techniques, in contrast to more automatic social information processing that normal developing individuals use, high-functioning people with ASD are able to pass complex emotion recognition and mentalizing tasks (Brosnan et al., 2015; Harms, Martin, \& Wallace, 2010; Loveland et al., 1997; Senju et al., 2009). Also, the demands of social interaction in daily life are not as explicitly defined as for example task instructions for social tests. Therefore, mental state attribution in high-functioning individuals with ASD may also be prompted by explicit task structure and instructions (Scheeren et al., 2013; Senju et al., 2009). A forced choice paradigm, as in the ERT, may facilitate the recognition of facial emotions, especially if the adolescents with ASD are formally trained to identify emotions using such labels. This kind of training is often a standard component of intervention programs and may have influenced the test results of the ASD group in the current study. Additionally, successful social interaction in daily life does not only depend on emotion recognition, but on the simultaneous integration of emotional information across modalities. Thus, it is possible that deficits in high functioning adolescents with ASD may only become apparent at the level of complex multisensory information processing (C. R. Jones et al., 2011).

Another additional explanation might be that high-functioning adolescents may not spontaneously be looking for, or detecting and using information from multiple simultaneous and dynamic sources in social situations, even though they might possess the ability to comprehend them (Grossman, Klin, Carter, \& Volkmar, 2000; C. R. Jones et al., 2011). They may avoid making eye contact, fixate on the mouth region (Neumann, Spezio, Piven, \& Adolphs, 2006), or miss a more general tendency to seek and initiate in social interactions. This may explain why high-functioning adolescents with ASD do not spontaneously attribute mental states, although they perform well on social functioning tasks. This is also consistent with the finding that training of mental state attributions does not necessarily improve social adaptation in ASD (Begeer et al., 2011; Loveland et al., 1997; S. Ozonoff \& Miller, 1995; Senju, 
2013), although some reports do show promising results (Bauminger, 2002; Stichter et al., 2010) of task-related transfer to various non-trained social situations.

Both explanations, i.e. the use of compensation techniques whether or not in combination with the lesser tendency to search for and use social information in more natural environments, may also explain why we do not find a relationship between our tasks and daily functioning levels; they may not measure the same cognitive or psychological construct. A limitation of the current study is the relatively smaller sample size, which may have limited our power for detecting possible significant differences between our groups. More research which can test the multimodal approach (and possible larger sample sizes) is needed to investigate whether social behavior and social cognition in high functioning adolescents with ASD are in fact two sides of the same coin. 



\section{4}

THE APPLICABILITY OF STANDARDIZED MEASURES OF EXECUTIVE FUNCTIONING IN HIGH-FUNCTIONING ADOLESCENTS WITH AN AUTISM SPECTRUM DISORDER (ASD)

Submitted for publication as:

Barendse, E.M., Hendriks, M.P., Thoonen, G., Kessels, R.P., Aldenkamp, A.P. The applicability of standardized measures of executive functioning in highfunctioning adolescents with an autism spectrum disorder (ASD). 


\section{Abstract}

Background: High-functioning adolescents with an Autism Spectrum Disorder (ASD) often show Executive Function (EF) deficits, which may contribute to or even underlie their ASD symptomatology. This study examines the relationship between ASD and EF in daily life and laboratory settings.

Methods: EF abilities of 24 high-functioning adolescents with ASD were compared with matched controls.

Results: The results showed that ASD adolescents showed more EF deficits than controls in daily life as reported by parents. However, these deficits could not be confirmed by neuropsychological measurements and were unrelated to ASD symptomatology as measured by the Autism Diagnostic Observation Schedule (ADOS).

Conclusion: The findings of this study are discussed in relation to the role of compensatory mechanisms and the ecological validity of neuropsychological tasks. 


\section{Introduction}

Autism Spectrum Disorder (ASD) is a heterogeneous neurodevelopmental syndrome, which is characterized by persistent deficits in social communication and social interaction across multiple contexts and restricted, repetitive patterns of behaviour, interest or activities (DSM-5). Despite continuous research, the aetiology of ASD is still unknown (Geschwind, 2011). The prevalence rate of ASD is high, estimated at 6 per 1000 children, with males being affected two to four times more often than females (Levy et al., 2009; A. O'Hare, 2009). Executive function problems are also found in individuals with ASD, but are not considered core deficits (Dawson et al., 2002; Geurts et al., 2004; Hill, 2004; O'Hearn et al., 2008; S. Ozonoff, Pennington, \& Rogers, 1991; Robinson et al., 2009). As executive functions (EFs) fulfil a central role in human cognition (Crone et al., 2009; Lezak, Howieson, Bigler, \& Tranel, 2012; Steinberg, 2005), adequate development of these functions and their underlying neural systems is necessary to become an independent adult.

Traditionally, EFs are described as a group of higher-order cognitive functions necessary for the regulation of voluntary planned behaviour and the inhibition of automatic task-inappropriate responses (O'Hearn et al., 2008). Pennington and Ozonoff (2010a) suggested five domains of EFs: inhibition, working memory, planning, cognitive flexibility and fluency. These processes are typically mediated by a fronto-parietal network, and deficits are seen in patients with brain damage in these regions (Bauminger, 2004; E. K. Miller \& Cohen, 2001). Impairments in EFs are also seen in neurodevelopmental disorders that are associated with prefrontal dysfunction, such as attention deficit hyperactivity disorder and autism (Bramham et al., 2009; Hill, 2004; O'Hearn et al., 2008).

Research on EFs in high-functioning individuals with ASD has shown behavioural problems in executive domains such as inhibition, planning, cognitive flexibility, verbal fluency, and visual working memory (Corbett et al., 2009; Geurts et al., 2004; K. A. Johnson et al., 2007; S. Ozonoff et al., 1991; Robinson et al., 2009; Steele et al., 2007; Verté et al., 2006a; Verté, Geurts, Roeyers, Oosterlaan, \& Sergeant, 2006b; Williams, Goldstein, \& Minshew, 2006; Zinke et al., 2010). During adolescence, the development of neural systems that sub-serve higher cognitive functions, like EFs, continues (Crone, Zanolie, Van Leijenhorst, Westenberg, \& Rombouts, 2008). The extent to which typically developing adolescents deploy these functions is highly flexible and depends on the social and motivational salience of the task demands at hand. Optimal executive function requires is often acquired through the natural tendencies of adolescents to approach, explore and experiment in other social situations, notably with their peers (Crone \& Dahl, 2012; Geurts \& Vissers, 2012; 
McAlonan et al., 2005; E. Pellicano, 2010b). Adolescents with ASD often lack this tendency to approach, explore and experiment or show it to a lesser extent (DSM-5). Moreover, children and adolescents with ASD may have a delayed developmental trajectory, which may result in a less sophisticated and flexible executive functioning in adulthood (Happé, Booth, Charlton, \& Hughes, 2006; Luna, Doll, Hegedus, Minshew, \& Sweeney, 2007; O'Hearn et al., 2008; E. Pellicano, 2010a). Also in elderly with ASD, deficits in sustained attention, working memory, and fluency have been reported (Bramham et al., 2009).

These executive difficulties may explain the rigidity, repetitive interests and the cognitive 'non-core' symptoms of ASD. However, studies on EFs in ASD show mixed results with respect to the (dys)executive profile that is found, that varies with respect to its strengths and weaknesses. While some authors demonstrated clear deficits in planning and flexibility in ASD (Craig et al., 2016), others reported unimpaired sustained attention, verbal fluency, response inhibition, planning or mental flexibility abilities in high-functioning individuals with ASD (Corbett et al., 2009; K. A. Johnson et al., 2007; E. Pellicano, 2010b; Robinson et al., 2009; Russell \& Hill, 2001).

Results from functional neuroimaging studies also identified various EF problems in individuals with ASD. Task-related functional neuroimaging studies showed deviations in activation patterns between high-functioning individuals with ASD and controls during inhibition and set shifting (Achenbach, 2011; Hofmann, Schmeichel, \& Baddeley, 2012; Kana, Keller, Minshew, \& Just, 2007), cognitive control (Solomon et al., 2009), working memory (Koshino et al., 2008; Luna et al., 2002), planning (Capps, Sigman, \& Yirmiya, 1995), fluency and mental flexibility tasks (Gilbert, Bird, Brindley, Frith, \& Burgess, 2008). In these studies, the ASD group either showed increased (Achenbach, 2011), decreased (Capps et al., 1995; Hofmann et al., 2012; Luna et al., 2002; Solomon et al., 2009) or EF task-dependent increased or decreased (Gilbert et al., 2008) activation patterns in prefrontal and parietal areas. Furthermore, differences in functional networks between high-functioning individuals with ASD and controls and evidence for hyper- and/or hypoconnectivity patterns in the high-functioning individuals with ASD were demonstrated in both prefrontal and more global brain networks (Barbey et al., 2012; Capps et al., 1995; T. B. Jones et al., 2010; Koshino et al., 2008; Luna et al., 2002; Pennington \& Ozonoff, 1996; Solomon et al., 2009). Assaf and colleagues (1996) found in their non-task related fMRI study a negative correlation between the hypoconnectivity and the severity of the adolescents' social and communication deficits as measured with the Autism Diagnostic Observation Schedule (ADOS) in high-functioning adolescents with ASD. Other studies also suggested that these altered activation and connectivity patterns were associated with the core symptoms of ASD (For review see: Just, Keller, Malave, Kana, \& Varma, 
2012). Still, it is unclear how these altered activation patterns and connectivity exactly relate to ASD symptoms in individuals.

Overall, despite the controversy about the neural correlates of EF deficits, these results indicate that executive dysfunction is an important contributor to maladaptive behaviour in high-functioning adolescents with ASD. Strikingly, despite the abundant research on EFs in high-functioning individuals with ASD, to our knowledge only a few studies have made a direct link between everyday dysexecutive behaviour and the performance on tests of executive functioning in adolescents with ASD (Chen et al., 2016; Happé et al., 2006). Happé and colleagues (2006), for example compared parent and teacher ratings of everyday adaptation, thus not executive functioning as such, with the performance on various EFs tests. They found relationships between interpersonal socialization, communication and socialization skills (Vineland Adaptive Behavior Scales) and the EFs response selection, flexibility and planning/ working memory. Although these studies related executive functioning in a test setting with everyday adaptation scores, they did not investigate the relation between EFs as measured in a test setting and executive functioning outside the laboratory. Others (2002; Granader et al., 2014; Lawson et al., 2015; Leung, Vogan, Powell, Anagnostou, \& Taylor, 2016; Pugliese et al., 2016; van den Bergh, Scheeren, Begeer, Koot, \& Geurts, 2014) have related executive functioning in daily life as measured with the Behavior Rating Inventory of Executive Function (BRIEF) with adaptive behaviour and found negative correlations between various subscales of the BRIEF and the adaptive skills of children with ASD. They concluded that impairments in executive functioning were strongly associated with the core deficits found in children with ASD. Despite these interesting results, these studies have several limitations. That is, control groups of typically developing children were not always included and no direct measurements of executive function were performed.

To address these limitations, the current study examines both real-life and performance- based abilities of EF in high functioning adolescents with ASD and a control group. Whilst the term High Functioning ASD (or HFA) is commonly used, it is not a formal subcategory of ASD. Intelligence levels within the high functioning population range between borderline (IQ between 70-79) and gifted (IQ above 145). As research showed that IQ has a different moderating effect on the presentation of ASD symptoms, especially with increasing age and intelligence levels (Lahuis et al., 2008), the subcategory HFA is somewhat arbitrary. Therefore, in the current study we only included high functioning adolescents with an IQ above 100. To the best of our knowledge, this is the first study to directly compare real-life and performance based EF abilities in adolescents with ASD with an (above) average intelligence level. The main purpose of this study is to examine executive functioning abilities in and outside 
the test setting by using parent ratings of executive functioning (BRIEF) and compare these results with task-related measurements of various EFs. Based on previous literature, we hypothesize to find executive function deficits in adolescents with ASD both in daily-life situations and in test settings. We do not expect to find global EF difficulties in this group, but an executive profile of both strengths and weaknesses, as described previously. Additionally, we explored the relationship between executive functioning and the core symptoms of ASD as measured with a standard ASD diagnostic tool, the Autism Diagnostic Observation Schedule (ADOS), especially since this topic is only sparsely described in literature.

\section{Methods}

\section{Participants}

Twenty-nine high-functioning adolescents (between 12 and 18 years of age) diagnosed with ASD (according to the DSM-IV criteria) were recruited from special secondary education school 'De Berkenschutse' located in Heeze, the Netherlands, as part of a larger study on working-memory problems in high-functioning adolescents with ASD. The study protocol was approved by the Medical Ethical Commission of the Maastricht University Medical Center.

Exclusion criteria for the ASD group were: (1) an (additional) diagnosis of a sychiatric disease as formulated in DSM-IV, such as attention-deficit or disruptive behaviour disorders, separation anxiety disorders, selective mutism, reactive attachment disorder of infancy or early childhood, anxiety disorders and mood disorders; (2) appearance of additional variables that can influence cognitive functioning such as pathology of the Central Nervous System, and (3) a significant visual or hearing impairment. Of the 29 recruited adolescents with ASD, twenty-four fulfilled both the established classification criteria according to DSM-IV, as well as the autism algorithm cut-offs on the ADOS. One participant did not return the BRIEF questionnaire and was excluded from this study. Eventually 23 adolescents (2 females) with autism disorder $(n=3)$, Asperger syndrome $(n=10)$, and pervasive developmental disorder not otherwise specified $(n=10)$ were included in all analyses. Eight adolescents with ASD used medication; six of them used methylphenidate or dexamphetamine, but discontinued medication at least 20-h before testing allowing for complete washout. Two adolescents with ASD used pipamperon/aripiprazol (an antipsychotic) and did not discontinue medication. 
Control adolescents were recruited through advertisements in local newspapers. The control group consisted of twenty-four typically developing adolescents (2 females), without a history of psychological or psychiatric disorders as confirmed by their parents/caregivers. Adolescents of both groups followed pre-university education or advanced general secondary education.

All participants had average to above-average estimated FSIQs [range ASD: 107-129; range controls: 105-135], as measured with the validated short version of the Wechsler Intelligence Scale for Children (WISC-III) (Campbell, 1998; Donders, 1997, 2001). The subtests used for this short version were Similarities and Vocabulary (as measures of the Verbal Comprehension Index)), Picture Completion and Block Design (as measures of the Perceptual Organization Index (POI)), Coding and Symbol Search (as measures of the Processing Speed Index), and Arithmetic and Digit Span (as measures of the Freedom from Distractibility Index (FDI)). Six of these subtest (excluding Digit Span and Symbol Search) were used to estimate the FSIQ (as described by Donders (Donders, 1997)). The two groups did not differ with respect to age $\left[F(1,45)=.44, p=.51, \eta_{p}^{2}=.01\right]$, Education level and years of education [respectively: $U=209, z=-1.80, p=.072, r=-0.26$ and $F(1,45)=.50, p=.49, \eta_{p}^{2}=.01$ ], Freedom from Distractibility Index (FDI) $\left[F(1,45)=2.68, p=.11, \eta_{p}^{2}=.06\right]$, and sex distribution. Although matched on education level, significant differences between the two groups were found with respect to the $\operatorname{VCI}\left[F(1,45)=8.01, p=.007, \eta_{p}^{2}=.15\right]$, POI $\left[F(1,45)=11.64, p=.001, \eta_{p}^{2}=.21\right]$ and $F S I Q\left[F(1,45)=12.64, p=.001, \eta_{p}^{2}=.22\right]$, with adolescents with ASD having higher estimated intelligence levels than controls, but effect sizes are small (Table 1).

\section{Procedure}

All participants who returned the informed consent form (adolescent and their parents/ caregivers) were asked to fill out and return questionnaires. To get a complete view of current functioning of the participants, several questionnaires were administered to assess competence levels, behavioural problems, emotional problems, and a global sense of self-esteem. In the current study, we used the Behavior Rating Inventory of Executive Function (BRIEF). The ADOS was conducted at all adolescents to confirm the diagnosis of ASD. All participants further participated in a neuropsychological assessment aimed to measure the various EF functions.

\section{Diagnostic assessments.}

The Autism Diagnostic Observation Schedule Module 4 (Lord et al., 2010) is a semi-structured, standardized assessment of social, communication, and language behaviour in adolescents and adults who are verbally fluent. During the 45-50-minute assessment, activities and unstructured conversation are combined with structured 
Table 1 Demographic and descriptive data

\begin{tabular}{|c|c|c|c|c|}
\hline & C & ASD & & \\
\hline \multirow[t]{3}{*}{ Gender (male/female) } & $22 / 2$ & $21 / 2$ & & \\
\hline & \multirow[t]{2}{*}{$M(s d)$} & \multirow[t]{2}{*}{$M(s d)$} & Group & \multirow[t]{2}{*}{$n_{p}^{2}$} \\
\hline & & & comparison & \\
\hline Age in months & $169.5(18.2)$ & $173.0(17.3)$ & $F(1,45)=.44, p=.51$ & .01 \\
\hline $\mathrm{VCl}$ & $114.6(9.2)$ & $122.3(9.3)$ & $F(1,45)=8.01, p=.007$ & .15 \\
\hline $\mathrm{POI}$ & $106.1(8.0)$ & $114.5(8.9)$ & $F(1,45)=11.64, p=.001$ & .21 \\
\hline FDI & $105.8(15.5)$ & $99.0(12.8)$ & $F(1,45)=2.68, p=.11$ & .06 \\
\hline FSIQ & $112.3(6.5)$ & $118.7(6.0)$ & $F(1,45)=12.64, p=.001$ & .22 \\
\hline
\end{tabular}

Note: Because we used a short version of the WISC-III, the Verbal Intelligence Quotient (VIQ) and the Performance Intelligence Quotient (PIQ) could not be calculated. $\mathrm{VCl}=$ Verbal Comprehension Index; $\mathrm{POI}=$ Perceptual Organization Index; FDI = Freedom from Distractibility Index; FSIQ = Full Scale Intelligence Quotient. $\mathrm{C}=$ Control group; ASD = ASD group.

situations and interview questions that offer the opportunity to observe a variety of behaviour. The goal of the ADOS module 4 is to determine how well the participant is able to initiate and sustain social interaction in situations with a minimum of structure and direction. Higher scores on the ADOS domains (Communication, Social interaction, and Stereotyped behaviour and restricted interests) indicate more problems on that specific domain.

The Behavior Rating Inventory of Executive Function (BRIEF; parent form) is a parentrating questionnaire that assesses behavioural manifestations of executive function abilities in children and adolescents (Gioia, Isquith, Guy, \& Kenworthy, 2009). The BRIEF is one of the few scales to measure everyday executive functioning in children and adolescents and is a well-designed, psychometrically sound instrument (Field, 2009; G. A. Miller \& Chapman, 2001). The BRIEF consists of eight clinical scales: Inhibit, Shift, Emotional Control, Initiate, Working memory, Plan/Organize, Organization of Materials and Monitor. In addition, two general indices (the Behavioral Regulation Index and Metacognition Index) and a total score (Global Executive Composite) are calculated. Higher scores on the BRIEF indicate more problems in executive functioning.

\section{Neuropsychological assessment.}

The overall study of which this study forms a part of enclosed an extended neuropsychological assessment of approximately three hours. All adolescents completed six 
tasks hypothesized to tap one of the executive functions as proposed by Pennington and Ozonoff (E. Pellicano, 2010a), as well as a sustained attention task. Tasks were selected based on earlier research and were administered in their Dutch versions. Commonly dependent variables were selected for each task:

Sustained selective attention: Bourdon-Vos test (Barnhill et al., 2000; Lezak et al., 2012). The Bourdon-Vos is a paper- and pencil cancellation test that measures sustained selective attention in terms of speed and accuracy. The participant should mark al 4-dot figures in a row, as fast and accurately as possible. The dependent measures in this task were the Mean completion time per row and its Standard deviation. A higher mean standard deviation on this test indicates more problems in sustained attention.

Inhibition: Test of Everyday Attention for Children Walk, Don't Walk subtest (Manly, Robertson, Anderson, \& Nimmo-Smith, 2004). The Walk, Don't Walk subtest is a go/ no-go paradigm and measures response inhibition. The dependent measure of this task was the Total number of correct trials.

Working memory (spatial and verbal span): Box task (Lord et al., 2010). The Box Task is a computerized visuospatial search task based on the principle of tasks such as the Executive Golf Task (Biederman et al., 2010). The participant has to search for hidden objects in a series of three, four, six or eight boxes. Two types of errors are possible; a within-search error: the participant returns to a previously opened box within a search. This measure reflects the ability to actively keep spatial information on-line during a search; second, a between-search error: the participant returns to a box which contains a target item from a previous search. This measure assesses the ability to maintain spatial information over longer periods of time in working memory. Dependent measures of this task were the number of between-search errors for the trials with four, six and eight boxes.

Digit span (Lezak et al., 2012; Liss et al., 2001). This task is a subtest of the WISC-III, and measures the verbal working memory span. In this task, the participant has to repeat digit sequences of increasing length in the correct forward or backward sequence. Dependent measures were the total number of correct responses in the forward and backward condition separately.

Cognitive flexibility (flexibility and shifting): D-KEFS Design fluency (Delis, Kaplan, \& Kramer, 2008; Lezak et al., 2012). This design fluency test was selected to measure flexibility and shifting, and has three conditions each allowing one minute for completion. In the first condition, the participant has to draw as many different 
patterns as possible in squares with five asymmetrically positioned filled dots. The second condition introduces interference with five additional unfilled dots that have to be ignored. The third condition is a switch condition in which the participant has to draw patterns alternating between filled and unfilled dots. The dependent measures in this task were a) Total number of correct patterns of condition one and two (flexibility); and b) Total number of correct condition three versus conditions one and two (shifting).

Planning: D-KEFS Tower Test (Lezak et al., 2012; Wechsler, 2005). This Tower Test is a variation of other Tower tests and is highly similar to the Tower of Hanoi ( $K$. A. Johnson et al., 2007), measuring planning and problem-solving abilities. The dependent measures of this test were the Total achievement score and the Move accuracy ratio. The Total achievement score is based on how many towers were correctly completed within the time limit and how many moves were required to complete them. The Move accuracy ratio represents the number of moves it took the participant to complete the items, compared to the minimal number of moves necessary to complete the items. This last score also gives an indication of inhibition abilities. A higher Total achievement score and a lower Move accuracy ratio are indications of better planning abilities.

\section{Statistical Analysis}

Data analysis was performed using the Statistical Package for Social Sciences (SPSS version 20.0). Raw scores were used in all analyses, unless otherwise indicated. Assumptions for parametric analyses were checked (normality, homogeneity of variance, correlations between dependent variables). These assumptions were not met for all variables, but we decided to perform (multivariate) analyses of variance ([M]ANOVA) rather than non-parametric tests, as the F-statistic is robust against violations of normality when group sizes are equal (as is the case in our study) (Donaldson, 1968). Also, there were significant differences between the two groups on $\mathrm{VCl}, \mathrm{POI}$ and $\mathrm{FSIQ}$; these variables were included as covariates in additional analyses to examine interactions between intelligence and executive function (i.e., the BRIEF and neuropsychological test scores), but not to statistically adjust for these differences (Dennis, Francis, et al., 2009; Field, 2009; G. A. Miller \& Chapman, 2001). To correct for the multiple comparisons only $p$-values less than 0.01 were interpreted as statistically significant.

\section{BRIEF.}

Groups were compared on daily-life EF abilities using MANOVAs, with group as independent variable and the various subscales of the BRIEF as dependent variables. To make it possible to examine interaction effects between intelligence and the BRIEF 
indices, we conducted three separate ANOVAs with group as independent variable, the z-scores of FSIQ as covariate, and the BRIEF Behavioral Regulation Index/ Metacognition Index / Global Executive Composite as dependent variable. The descriptive data of the standard (t-scores) of the BRIEF were used to examine the percentage of adolescents at or above the borderline range for the various scales (t-score higher than or equal to 60 ).

\section{Neuropsychological assessment.}

The dependent measures were analysed using ANOVAs with group as between-subject factor. In case of more than one dependent variable within a task, MANOVAs were conducted instead of an ANOVA. To make it possible, despite group differences, to speculate about the possible influence of intelligence on EF, we also conducted two separate ANOVAs with group and the z-scores of FSIQ as independent variables, and the various EF scores as dependent variable.

Correlations (Pearson correlation coefficient) between the BRIEF and the executive functioning tasks were calculated to investigate the relationship between executive test performance and everyday executive functioning. These correlations were compared for both groups separately. The alpha level was adjusted (Bonferroni correction) to correct for multiple comparisons.

\section{ADOS.}

Finally, exploratory analyses were performed between the four ADOS domains, the BRIEF subscales and the scores on the EF tasks in the ASD group.

\section{Results}

\section{BRIEF}

As predicted, the MANOVA revealed a significant main effect of group on the BRIEF [Wilks $\Lambda=.31, F(8 ; 38)=10.43, p<.001, \eta_{p}^{2}=.69$ ], with adolescents in the ASD group having higher mean scores on the BRIEF than Controls. Subsequent ANOVAs showed significant group effects on all scales, with noteworthy high effect sizes for Shift $\left[F(1,45)=69.11, p<.001, \eta_{p}^{2}=.61\right]$, Initiate $\left[F(1,45)=48.61, p<.001, \eta_{p}^{2}=.52\right]$, Plan/Organize $\left[F(1,45)=48.66, p<.001, \eta_{p}^{2}=.52\right]$, Monitoring one's own behavior $\left[F(1,45)=65.23, p<.001, \eta_{p}^{2}=.59\right]$, the Metacognition Index $[F(1,45)=66.91, p<.001$, $\left.\eta_{p}^{2}=.60\right]$ and the Global Executive Composite on the BRIEF $[F(1,45)=68.44, p<.001$, $\left.\eta_{p}^{2}=.60\right]$ (Table 2). Parents of adolescents with ASD reported more problems in executive functioning in daily situations, than parents of control adolescents. 
Significant interaction effects were found between group and FSIQ in relation to the BRIEF Behavioral Regulation Index $\left[F(1,43)=7.43, p=.009, \eta_{p}^{2}=.15\right.$; Control $b_{0}=-1.10$, ASD $\left.b_{0}=7.16\right]$, BRIEF Metacognition Index $F(1,43)=4.16, p=.048, \eta_{p}^{2}=.09$; Control $\left.b_{0}=-4.44, A S D b_{0}=4.29\right]$, and BRIEF Global Executive Composite $F(1,43)=6.73$, $p=.013, n_{p}^{2}=.14$; Control $b_{0}=-5.54$, ASD $\left.b_{0}=11.44\right]$. A significant interaction effect was also found between group and $\mathrm{VCl}$ in relation to the BRIEF Behavioral Regulation Index $\left[F(1,43)=5.82, p=.02, \eta_{p}^{2}=.12\right.$; Control $\left.b_{0}=-.60, A S D b_{0}=6.42\right]$. No interaction effects were found between $\mathrm{VCl}$ and the BRIEF Metacognition Index or Global Executive Composite, and POI or FDI and the BRIEF Behavior Regulation Index/ Metacognition Index /Global Executive Composite. Thus, parents of adolescents with ASD reported more problems in executive functioning if their child had a higher Full-Scale IQ, while parents of control adolescents reported fewer problems in executive functioning if their child had a higher FSIQ.

In the ASD group, more than half of the adolescents scored on or above the 'borderline' cut-off score on the subscales Shift (65.2\%, T-score range 45-80), Initiate (65.2\%, T-score range 44-77), and on Monitor (56.5\%, T-score range 44-75). Percentages were somewhat lower on the other subscales, but still substantial (Inhibit 47.8\%, T-score range 39-73, Emotional control 34.8\%, T-score range 38-83, Working memory $34.8 \%$, T-score range $41-73$, Plan/Organize $21.7 \%$, T-score range 34-87, Organization of Materials 17.4\%, T-score range 35-74, Behavioral Regulation Index 47.8\%, T-score range 43-83, Metacognition Index 43.5\%, T-score range 39-75, Global Executive Composite $43.5 \%$, T-score range 40-75). In the Control group, one adolescents scored on the 'borderline' cut-off score of the subscale Initiate (4\%, T-score range 30-60), but none of the adolescents scored on or above the cut-off score for any of the other BRIEF scales.

\section{Executive Function tasks}

In contrary to our predictions, both groups did not significantly differ on most measurements of the executive function tasks, except on the mean standard deviation scores of the Bourdon-Vos task $\left[F(1,45)=5.418, p=.024, n_{p}^{2}=.107\right]$ (Table 3), on which the ASD group had higher scores (more problems sustaining their attention) than the control group. An interaction effect was found between group and FSIQ for the Box-task 6 boxes $\left[F(1,43)=31.41, p<.000, \eta_{p}^{2}=.42\right.$; Control $b_{0}=1.77$, ASD $\left.b_{0}=-.22\right]$. No significant interaction effects were found between group and $\mathrm{VCI}, \mathrm{POI}$ or FDI for any of the executive tasks. This indicates that adolescents with ASD perform slightly worse on a sustained attention task, but on average, do not show impaired executive functioning compared to typically developing adolescents. 
Table 2 Group comparisons on the BRIEF

\begin{tabular}{|c|c|c|c|c|}
\hline & \multicolumn{2}{|c|}{ Group } & & \\
\hline & C & ASD & & \\
\hline \multirow[t]{2}{*}{ Gender (male/female) } & $22 / 2$ & $21 / 2$ & & \\
\hline & $M(s d)$ & $M(s d)$ & $p$ & $\eta_{p}^{2}$ \\
\hline Inhibition & $11.75(2.23)$ & $17.61(4.79)$ & $<0.001$ & .39 \\
\hline Shifting & $9.67(2.04)$ & $16.78(3.64)$ & $<0.001$ & .61 \\
\hline Emotional control & $11.67(2.10)$ & $17.30(5.66)$ & $<0.001$ & .32 \\
\hline Initiation & $12.17(2.96)$ & $18.52(3.30)$ & $<0.001$ & .52 \\
\hline Working memory & $13.29(3.01)$ & $20.43(4.63)$ & $<0.001$ & .47 \\
\hline Planning and organization & $16.54(2.98)$ & $24.22(4.45)$ & $<0.001$ & .52 \\
\hline Orderly & $9.42(2.48)$ & $12.52(3.15)$ & $<0.001$ & .24 \\
\hline Monitoring own behavior & $12.33(2.71)$ & $19.35(3.23)$ & $<0.001$ & .59 \\
\hline BRI & $33.08(5.27)$ & $51.70(13.04)$ & $<0.001$ & .48 \\
\hline MI & $63.75(10.68)$ & $95.04(15.24)$ & $<0.001$ & .60 \\
\hline Total score & $96.83(14.61)$ & $146.74(25.52)$ & $<0.001$ & .60 \\
\hline
\end{tabular}

Note: $\mathrm{C}=$ Control group; $\mathrm{ASD}=\mathrm{ASD}$ group. $\mathrm{BRI}=$ Behavior Regulation Index; $\mathrm{MI}=$ Metacognition Index .

Correlation analyses on the BRIEF and EF tasks for both groups separately did not show any significant correlations in the ASD group. In the control group, significant correlations were found between the Cancellation task (mean reaction time) and the BRIEF Shift $[r=.521, p=.009]$ and Emotional control $[r=.540, p=.006]$ subscales, and between the Tower and the BRIEF Inhibit subscale [Total no correct: $r=-.572, p=.003$; and Move accuracy ratio: $r=.558, p=.005]$.

Of the ASD group, all adolescent scored on or above cut-off levels on the domains of Communication $[\mathrm{m}=4.00$, range $=2-6]$, Reciprocal Social Interaction $[\mathrm{m}=6.78$, range $=4-11]$ and Total ADOS score $[m=10.83$, range $=7-17]$. The ADOS domain Stereotyped Behaviors and Restricted Interests has no cut-off level $[\mathrm{m}=1.04$, range $=$ 0-3]. One-tailed correlation analyses on the four ADOS domain scores and the BRIEF subscales and/or the EF tasks showed no significant correlations. 


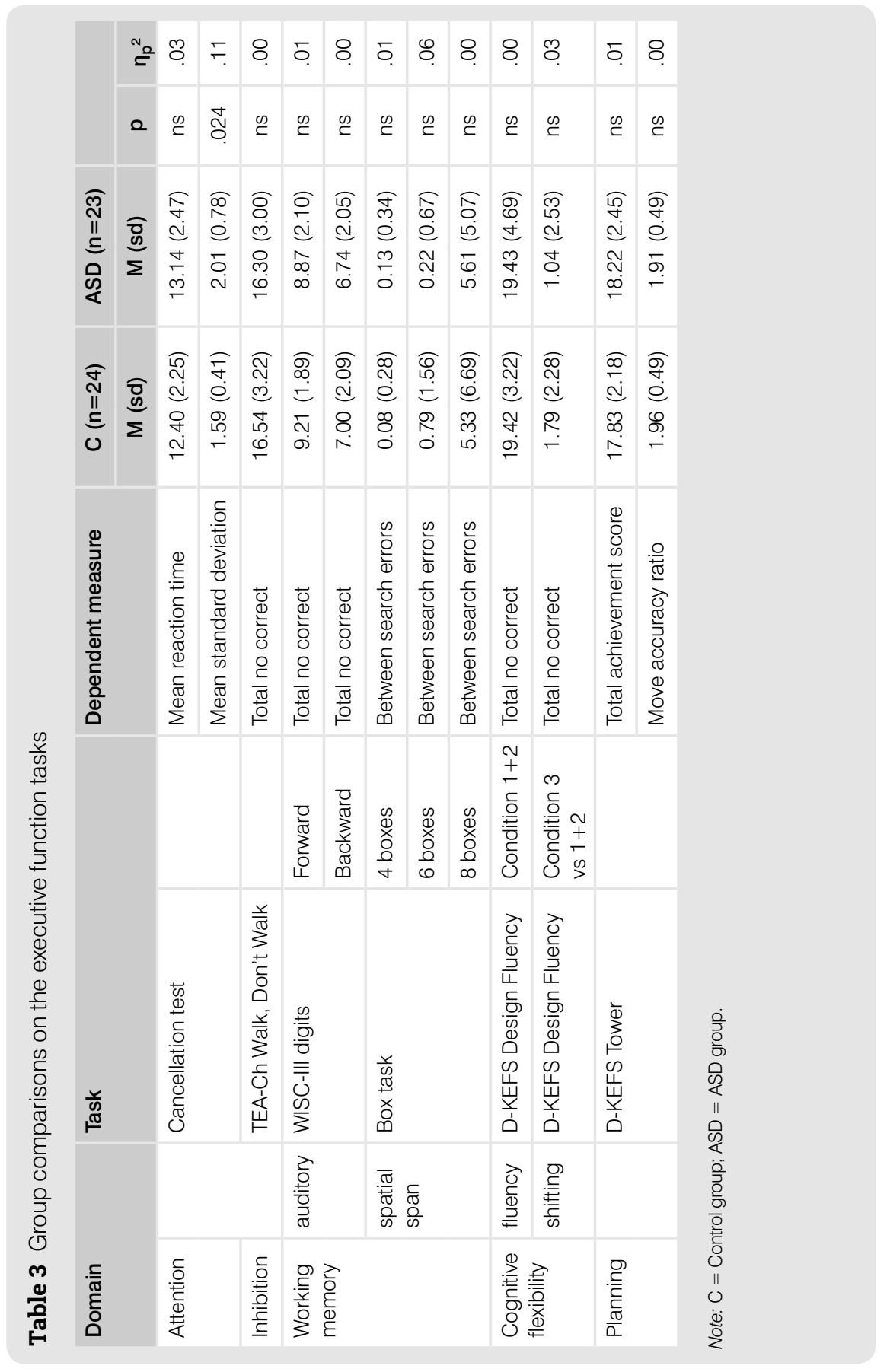




\section{Discussion}

Although previous studies showed that executive function deficits are common in high-functioning adolescents with ASD, so far no study has directly compared real-life functioning and performance based EF abilities in adolescents with ASD. Therefore, the major aim of this study was to assess EF abilities of high-functioning adolescents with ASD and an (above) average intelligence both in real-life and in laboratory settings (using neuropsychological tasks). As a second aim, we examined whether these abilities were associated with the core symptoms of ASD; that is, social impairments, communication difficulties and repetitive interests and behaviour. The current results suggest that, as reported by parents, adolescents with ASD and an (above) average intelligence show EF difficulties in various domains in everyday situations. However, these deficits could not be substantiated with the performance on neuropsychological tests. Neither the BRIEF nor the EF tasks showed a significant relationship with ASD symptomatology.

These seemingly contradictory results have been suggested in other studies and are often seen as the result of compensatory strategies. In laboratory settings, due to the highly structured setting and protocol, high-functioning adolescents with ASD may be able to cognitively compensate for their EF shortcomings (Gilotty et al., 2002; Wing, 1997). In real life, however, (social) information processing does not follow a structured protocol. These situations are often complex and unpredictable, and require fast and flexible information processing. High-functioning individuals with ASD might not be able to compensate for their problems in these real-life situations, which may explain the EF deficits as often described by parents and other caregivers. Functional neuroimaging studies on EF in high-functioning individuals with ASD also attributed altered brain activation to the use of alternative compensatory cognitive strategies (Achenbach, 2011; Bramham et al., 2009; Kana et al., 2007; Koshino et al., 2008). These alternative cognitive strategies may also explain why in some fMRI studies no behavioural differences on a task level were found, despite altered brain activation or connectivity.

With respect to the use of compensation strategies, these techniques are mainly reported in high-functioning children or adolescents with ASD. Due to their average or higher intelligence, they can, for example, learn cognitive strategies or the 'rules' of social interaction by rote, although they lack the intuitive flexibility through which typically developing children or adolescents 'learn' and apply these (Asperger, 1943; Bramham et al., 2009; Hill \& Frith, 2003; E. Pellicano, 2010a; Wing, 1997). In addition, this relationship between intelligence and using cognitive strategies is often described, where a higher intelligence is generally related to better executive 
functioning in both clinical (Mahone et al., 2002; Vriezen \& Pigott, 2002) and control groups (Friedman et al., 2006; Verhulst \& van der Ende, 1992). However, this effect seems to be less prominent in children with average or above-average intelligence levels compared with children with superior intelligence levels [i.e., a FSIQ > 130] (Verhulst \& van der Ende, 1992). Neuroimaging research showed that both general intelligence and EF recruit a highly overlapping and broadly distributed network of fronto-parietal regions (Bauminger, 2004). This neural as well as conceptual (Sukhodolsky et al., 2008) overlap is, however, not complete, which is also reflected in behavioural studies showing that intelligence is a moderator for EF, but in itself explains only $10 \%$ of the variance (Friedman et al., 2006; Verhulst \& van der Ende, 1992; Vriezen \& Pigott, 2002).

In our study, we found a small moderating effect of intelligence in relation to executive functioning as measured with the BRIEF. As in previous studies, this relationship was positive between intelligence and executive functioning in the control group. However, we found the opposite effect for adolescents with ASD, with parents of more intelligent adolescents reporting more EF deficits. A possible explanation could be the aforementioned measurement discrepancies between the BRIEF and the EF tasks. The moderation effect of intelligence on executive abilities could be different for the BRIEF and the EF tasks. Another hypothesis is that because the adolescents with ASD in our study are verbally high-functioning, higher expectations of parents about their executive abilities rise and as a consequence these youngsters may be structurally overestimated (Elsabbagh et al., 2012).

Another explanation for the contradicting results between the BRIEF and EF task measurements might be a more methodological one. Although the BRIEF is thought to measure EF abilities in real life situations, one might question whether it is really measuring specific cognitive EFs or that the BRIEF is a more general index of behavioural disruption and impairment (Vriezen \& Pigott, 2002). Although generally used in clinical practice for this purpose, the professional manual (Gioia et al., 2009) states that the BRIEF is validated with behaviour questionnaires such as the Child Behavior Checklist, but it does not include research on task-related measures of EF. In our study, beside a significant relationship between planning and the BRIEF Inhibit subscale in the control group, no significant relationships were found between EF task measurements and the BRIEF. Furthermore, recent research argued that the BRIEF is also not always related to objective EF task performance in other clinical groups, such as youth with traumatic brain injury, epilepsy, Attention Deficit/ Hyperactivity Disorder, Tourette syndrome, or spina bifida (for a nice overview see: McAuley, Chen, Goos, Schachar, \& Crosbie, 2010). Further research should give 
more insight in the precise relationship between the BRIEF, EF task performance and executive abilities in real-life situations.

The second aim of this study was to examine the relationship between EF abilities (both in real-life and laboratory situations) and ASD core symptoms as measured with the ADOS. Although executive function deficits have been hypothesized to contribute to ASD symptomatology and especially adaptive behaviour and perseveration (Larochette, Benn, \& Harrison, 2009), it is unclear whether this reasoning is causal, or more circular by nature. That is, EF-related symptoms such as perseveration may be a reflection of how autism was defined in the first place rather than a contributing factor to ASD symptomatology. In the current study, contrary to our predictions, EF tasks performance of the adolescents with ASD were not impaired, although parents reported EF deficits in real life. In line with the hypothesis that high-functioning adolescents with ASD may be able to compensate for their EF deficits in structured settings, objective tests for EF may not be sensitive enough to determine EF deficits (Delis, Kaplan, \& Kramer, 2007) and relate these to ASD symptoms. However, as parents reported many EF deficits, we expected to find a relation between EF functioning in real life and ASD symptoms. However, this relation was not present either, which supports the hypothesis that the often-reported EF difficulties in adolescents with ASD are related to general measures of behavioural disruption and impairment rather than to actual cognitive impairments in executive functioning.

In all, our results indicate that a developmental delay in individuals with ASD may induce compensatory mechanisms (Bramham et al., 2009), which in combination with a lesser or missing tendency to approach, explore and experiment in peer and social situations, may lead to a less sophisticated and flexible use of EFs in adolescents and adults with ASD in real life. In structural settings EF performance may be unimpaired. This raises questions about the ecological validity of standard EF task performance in adolescents with ASD with an (above) average intelligence. An hypothesis that will need more research is that the core ASD impairments in this specific subgroup are in essence behavioural in nature, but do not include cognitive impairments, specifically impairments of EF. 



\section{REVIEW: WORKING MEMORY DEFICITS IN HIGH-FUNCTIONING ADOLESCENTS WITH AUTISM SPECTRUM DISORDERS: NEUROPSYCHOLOGICAL AND NEUROIMAGING CORRELATES}

\section{Published as:}

Barendse, E.M., Hendriks, M.P., Jansen, J.F., Backes, W.H., Hofman, P.A., Thoonen, G., Kessels, R.P., Aldenkamp, A.P. (2013).

Working memory deficits in high-functioning adolescents with autism spectrum disorders: neuropsychological and neuroimaging correlates.

Journal of Neurodevelopmental Disorders, 5 (1),14. doi: 10.1186/1866-1955-5-14. 


\section{Abstract}

Background: Working memory is a temporary storage system under attentional control. It is believed to play a central role in online processing of complex cognitive information and may also play a role in social cognition and interpersonal interactions. Adolescents with a disorder on the autism spectrum display problems in precisely these domains. Social impairments, communication difficulties, and repetitive interests and activities are core domains of autism spectrum disorders (ASD), and executive function problems are often seen throughout the spectrum. As the main cognitive theories of ASD, including the theory of mind deficit hypotheses, weak central coherence account, and the executive dysfunction theory, still fail to explain the broad spectrum of symptoms, a new perspective on the aetiology of ASD is needed. Deficits in working memory are central to many theories of psychopathology, and are generally linked to frontal-lobe dysfunction. This article will review neuropsychological and (functional) brain imaging studies on working memory in adolescents with ASD.

Conclusion: Although still disputed, it is concluded that within the working memory system specific problems of spatial working memory are often seen in adolescents with ASD. These problems increase when information is more complex and greater demands on working memory are made. Neuroimaging studies indicate a more global working memory processing or connectivity deficiency, rather than a focused deficit in the prefrontal cortex. More research is needed to relate these working memory difficulties and neuroimaging results in ASD to the behavioural difficulties as seen in individuals with a disorder on the autism spectrum. 


\section{Introduction}

Autism spectrum disorder (ASD) is a heterogeneous neurodevelopmental syndrome in the category of pervasive developmental disorders (Diagnostic and Statistical Manual of Mental Disorders, 4th Edition (DSM-IV)), with an unknown aetiology. Clinicians and researchers usually include autistic disorder, Asperger syndrome, and pervasive developmental disorders not otherwise specified as subtypes of ASD. Although not part of the diagnostic classification and not formal subcategories of ASD, a distinction is also often made between low-functioning autism (LFA) and high-functioning autism (HFA). No consensus criteria regarding LFA and HFA exist, but low-functioning individuals with autism are generally considered to have an IQ below 70 or 85 , whereas high-functioning individuals with autism tend to have an IQ above 70 or 85 (depending on the preferred IQ cut-off point of one or two standard deviations below mean).

Although estimates vary, the prevalence rate of ASD is approximately 6 per 1,000 children, with males being affected two (autism disorder) to four (Asperger syndrome) times more often than females (Levy et al., 2009). Across the spectrum, ASD is by definition characterized by social impairments, communication difficulties, and repetitive interests and behaviour (DSM-IV). Executive function problems and (specific) learning difficulties are also found throughout the spectrum, but are not seen as core deficits (Dawson et al., 2002; Geurts et al., 2004; Hill, 2004; O'Hearn et al., 2008; S. Ozonoff et al., 1991; Robinson et al., 2009). Traditionally, 'executive functions' is an umbrella term for functions such as working memory (WM), inhibition, planning, impulse control, and shifting set as well as the initiation and monitoring of action (Hill, 2004). As such, executive functions are generally seen as central to human cognition (Crone et al., 2009; Lezak et al., 2012; Steinberg, 2005) and as a consequence, executive dysfunction has a high impact on daily life.

Of these executive functions, WM and inhibition are generally considered to be core components (S Ozonoff \& Strayer, 2001; Verté et al., 2006b). Although the precise definition of WM may depend on the field of interest, for example, in human or non-human studies (Owen, McMillan, Laird, \& Bullmore, 2005), it is generally considered to be a temporary storage system under attentional control that is the basis for our capacity for complex thought (Baddeley, 2012). This view is supported by abundant empirical studies revealing relationships between WM and reading, mathematics (Bull \& Andrews Espy, 2006; Cain, 2006; Jong de, 2006; Swanson, 2004), reasoning and problem-solving (Crone et al., 2009; Jarrold \& Towse, 2006; Kane et al., 2004), and general fluid intelligence (Gray, Chabris, \& Braver, 2003; Unsworth, Redick, Heitz, Broadway, \& Engle, 2009). The processes involved in WM 
are threefold: active conservation of information for short periods of time, contextrelevant updating of information, and rapid biasing of task-relevant cognitions and behaviours.

Nowadays, the most commonly used cognitive model of WM is the revised WM model proposed by Baddeley (Baddeley, 2012) (Figure 1). This model contains a central executive (attention control centre), an episodic buffer (which comprises a limited capacity storage system and integrates information into coherent objects and episodes) and two slave systems: one for visual and/or spatial information (the visuospatial sketchpad) and one for auditory information (the phonological loop). The model also describes links between WM, long-term memory, and perception, and has proven to be very valuable in WM studies that focus on abstract information processing, such as for numbers, locations of objects, words, and sentences (Baddeley, 2012), the so-called 'cold' (based on reason) cognitive processes. However, based on this revised WM model of Baddeley, we argue that WM is not only important for 'cold' cognitive processes (auditory and visuospatial), but is also essential for successfully navigating in the social world. Complex social situations demand online information processing of both 'cold' and 'hot' (based on motivation and emotion) cognitive information, and put a lot of stress on WM capacity (Brüne, 2003; E. K. Miller \& Cohen, 2001; Weissman, Perkins, \& Woldorff, 2008). In addition, Baddeley states that adequate social behaviour can be seen as a dual task, through balancing one's own needs and desires with those of the people with whom one is interacting, and as such requires a form of WM (Baddeley, Della Sala, Papagno, \& Spinnler, 1997). Although this last notion was proposed more than a decade ago, only a few studies have considered this subject, which will be discussed below.

LoPresti et al. (2008) examined how continuously changing social information is maintained in WM. They used two versions of a delayed match-to-sample task to examine two socially salient cues: emotion and identity. Participants either had to match the emotional expression of a face to a sample face (the identity of the face was constant), or match the identity of a face to a sample face (the emotional expression was constant). Their results demonstrated sustained delay-related activation in the orbitofrontal cortex, the amygdala, and hippocampus, for both identity and emotion. These regions play a critical role in binding together emotional information in long-term memory, and are also critical for actively maintaining and binding together salient social cues in WM (Bergmann, Rijpkema, Fernández, \& Kessels, 2012). Other evidence for the involvement of WM in processing social information was given by Phillips and colleagues (Phillips, Channon, Tunstall, Hedenstrom, \& Lyons, 2008). They explored the role of verbal working memory in decoding emotions and found that the process of labelling the emotions portrayed 


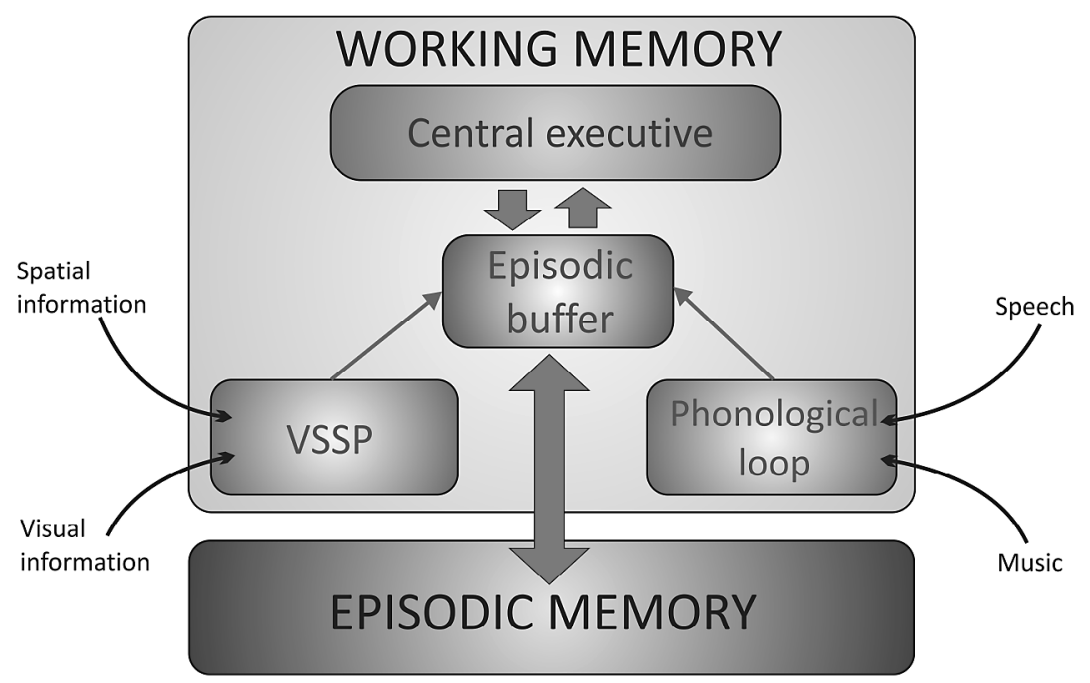

Figure 1 Baddeley's (2012) working memory model.

Note: VSSP = visuospatial sketchpad.

on facial expressions places high demands on WM resources. These demands increase as the number of labels that are available increases, as will be the case in real-life situations: decoding social cues from faces, bodies, and verbal material will all involve substantial investment of WM for accurate performance. The authors further mentioned that possible critical factors that might influence the WM load may be the type of decision required, the extent to which the social properties of the stimuli can be easily detected and verbalized, and the complexity of the stimuli and response requirements. Bankó and colleagues (Banko, Gal, \& Vidnyanszky, 2009) found evidence that humans can store fine-grained information related to facial emotions and identity in short-term memory with high precision. Such high-fidelity short-term memory processing is crucial for the ability to monitor emotional expressions efficiently. As a result, it could be hypothesized that impairment of high-precision short-term memory storage of emotional information might (partly) underlie the deficits of emotional processing found in psychiatric disorders, including autism. Through these processes, WM not only plays a central role in processing complex cognitive information, but also an essential role in social cognition and interpersonal interactions.

During adolescence major changes take place in the neural subsystems that serve social cognition, WM, and other executive functions (Blakemore \& Choudhury, 2006). 
Adolescence is a period in which social-cognitive skills become increasingly important. To achieve adult social competence levels, adolescents have to learn how to adapt their behaviour to rapidly changing social environments, in which the opinions and evaluations of peers become increasingly salient (Crone \& Dahl, 2012; Steinberg, 2005). Both social rule use and WM are likely to develop slowly and reach maturity in adolescence or early adulthood (Blakemore, 2008; Huizinga, Dolan, \& van der Molen, 2006; Nelson \& Guyer, 2011). The simultaneous development of both higher cognitive and socio-affective brain systems during adolescence, and the interplay of these systems on each other (Nelson, Leibenluft, McClure, \& Pine, 2005), makes this period especially interesting to study. While we acknowledge that the brain-behaviour relationship of social-cognition in adolescence is complex, with many interacting processes such as changing hormone levels during puberty, as well as motivational and environmental factors, we do not focus on these specific processes in this review. Readers are referred elsewhere for excellent empirical evidence and theoretical discussions on these topics (Crone \& Dahl, 2012; Nelson \& Guyer, 2011; Pfeifer \& Allen, 2012).

Despite the central role of WM in social cognition and in many, if not all, higher cognitive functions, the developmental tract of WM and the often reported executive function problems, few researchers have studied WM functioning in adolescents with ASD. Theory of mind deficit hypotheses, weak central coherence account, and the executive dysfunction theory all focus on certain symptoms of ASD (see Appendix-1) but fail to explain the broad spectrum of symptoms found in ASD. Also other theories have been proposed that try to explain ASD symptomatology, such as the mirror neuron theory (for a review see (Hamilton, 2013)) and the mnesic imbalance theory (Romero-Munguía, 2011); however, evidence for these theories is still inconclusive. A more recent hypothesis proposed by Happé and Ronald suggests that no single unitary account will be able to explain both social and non-social features of ASD. Instead they propose a fractionable triad approach, stating that, phenotypically and genetically, variations in (the triad of) social impairments, communication difficulties, and repetitive interests and behaviour, are considerably independent (Happé et al., 2006; F. Happe \& Ronald, 2008). Although we agree with Happé and Ronald that no unitary account will be able to explain the broad phenotype of ASD, we disagree that cognitive deficits (such as WM deficits) would not be able to explain the social side of the triad of symptoms (F. Happe \& Ronald, 2008). The underlying mechanisms of this disorder are still unknown and, considering the impact of ASD on many peoples' lives, new ways to access the cognitive and behavioural problems associated with ASD are needed. Deficits in WM and inhibition are central to many theories of developmental psychopathology (Verté et al., 2006b) and earlier research strongly suggests relationships between WM capacity impairment and cognitive and social 
problems in adolescents with ASD, attention deficit hyperactivity disorder, traumatic brain injury, and young adults with schizophrenia (Brüne, 2003; Dennis, Agostino, Roncadin, \& Levin, 2009; Gilotty et al., 2002; Toplak, Bucciarelli, Jain, \& Tannock, 2009; Verté et al., 2006b). Bull and colleagues (Bull, Phillips, \& Conway, 2008) found that executive processes, including WM, are required in both simpler and complex theory of mind tasks, underlining the role of WM in social skills. These findings may shed new light on what may be the underlying mechanisms of autism.

This review will focus on the neuropsychological results of WM studies in highfunctioning adolescents with ASD, as well as the possible correlates with neuroimaging studies in this population.

\section{Neuropsychological studies on working memory in high-functioning ASD individuals}

Although both theory and empirical findings suggest an interrelationship between WM and social cognition, the number of studies on WM functioning in adolescents with ASD is limited and findings are inconsistent (for an overview see Table 1). Some neuropsychological studies have failed to find any significant group differences between high-functioning adolescents with autism and normal controls on WM tasks (Geurts et al., 2004; Happé et al., 2006; S Ozonoff \& Strayer, 2001; Sinzig, Morsch, Bruning, Schmidt, \& Lehmkuhl, 2008). Nonetheless, a number of other studies clearly demonstrate impairments associated with WM and other executive functions in high-functioning adolescents with autism. Most of these impairments are found in the spatial domain of WM. Minshew and colleagues, for example, repeatedly found reduced spatial WM abilities in adolescents with autism (Luna et al., 2007; Steele et al., 2007; Williams, Goldstein, Carpenter, \& Minshew, 2005; Williams et al., 2006). Other research groups have also found spatial WM problems in adolescents with ASD (Corbett et al., 2009; Goldberg et al., 2005; Landa \& Goldberg, 2005; Verté et al., 2006a).

All of the abovementioned studies used well-known and validated tasks such as the WRAML finger windows and CANTAB spatial span and spatial WM, and related the number of errors to difficulties in WM mechanisms. Two studies used different and more experimental research designs. In an oculomotor delayed response task, Luna et al. (2007) found a developmental delay of WM in adolescents with ASD and a less refined use of WM in adults with ASD. The results indicate that WM problems are present in adolescence and despite an increase in capacity during development, adult levels of WM capacity are both reduced and delayed in ASD compared to 


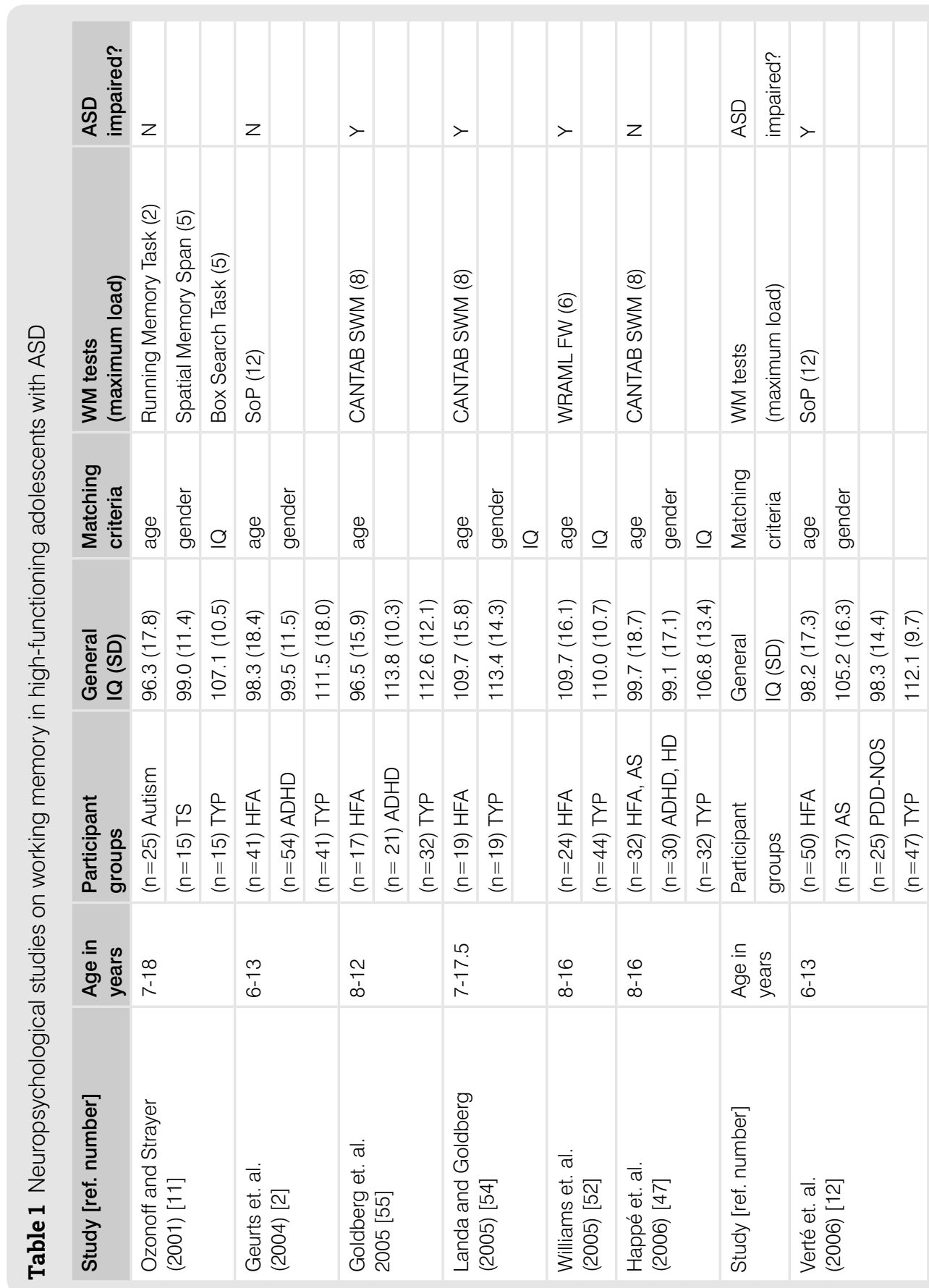




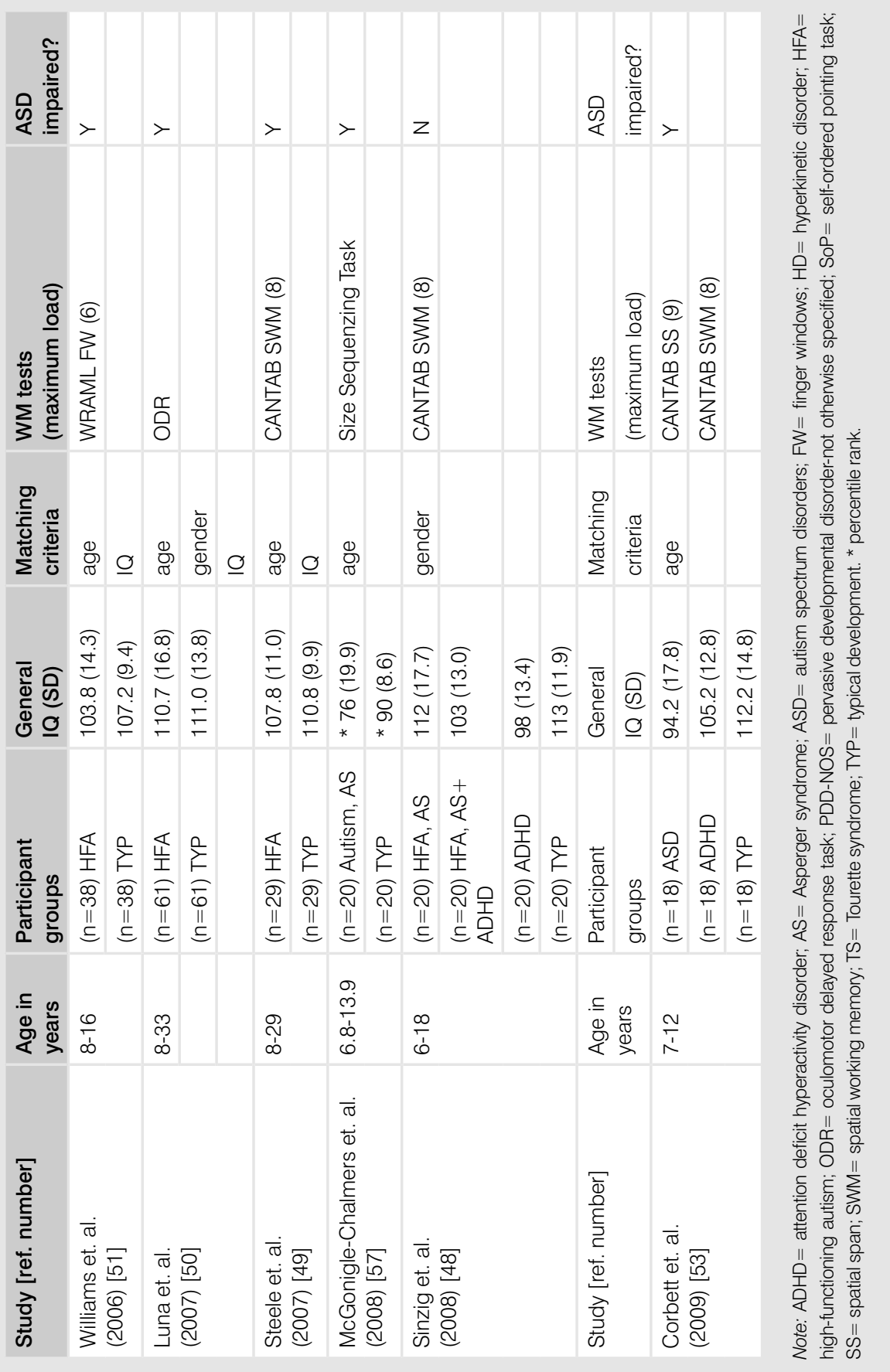


controls. In the study by McGonigle-Chalmers (McGonigle-Chalmers, Bodner, Fox-Pitt, \& Nicholson, 2008) a size-sequencing task was used, in which participants had to sort randomly presented stars on a touch screen in either a descending or ascending order. The authors found that adolescents with ASD not only made more errors but also had higher reaction times than healthy controls. Because of the strong bias in the error data of all participants towards 'forwards' rather than 'backwards' errors, they concluded that the higher error rate and reaction times of adolescents with ASD can best be explained by deficits in the prospective component of WM responsible for the context-relevant updating of information, and rapid biasing of task-relevant cognitions and behaviours. These processes may also play important roles in the adjustment of behaviour to often rapid and continuously changing social situations.

In most studies, WM problems increase when tasks impose heavier demands on working memory, for instance, when more complex or social information has to be processed (Landa \& Goldberg, 2005; McGonigle-Chalmers et al., 2008; Williams et al., 2006). In the CANTAB spatial WM task, Landa and Goldberg (2005) found that children and adolescents with ASD made more between-search errors than non-ASD controls on the search task with six and eight boxes, but not on the one with four boxes. A between-search error is made when the child returns to a spatial location (box) that contains a - now irrelevant - target from a previous search. Interestingly, the number of between-search errors made by the children with ASD were related to a greater social dysfunction on the autism diagnostic observation schedule (ADOS). Similarly, Williams et al. (2006) reported more WM problems in children and adolescents with ASD on the more complex design memory and picture memory subtest of the WRAML, whilst finding no WM problems on a less complex number/ letter WM task.

These memory-load specific effects may explain why other studies did not find WM problems in adolescents with ASD. For example, the spatial span that Sally Ozonoff and Strayer used in their spatial memory-span task (2001) had only a limited working-memory load. Participants had to remember the location of one, three or five coloured geometric shapes over a 1,000 or 5,000 ms delay. This low task load on WM did not reveal any difficulties. In other WM studies, problems only appeared when six or eight stimuli had to be memorized (Landa \& Goldberg, 2005; Steele et al., 2007). Thus, a task using a maximum of five stimuli might simply not uncover spatial WM problems in high-functioning adolescents with ASD. Geurts and colleagues (2004) failed to find an increase in working memory problems with increasing WM load on a self-ordered pointing task in high-functioning adolescents with ASD compared with a control group. However, as they compared both groups on differences in beta 
weights (with difficulty level as the predictor and the number of errors as the dependent variable), no conclusions can be drawn about possible differences between the groups regarding the number of errors made on each load $(6,8,10$, or 12 items). Presumably, the stress placed on the WM system plays an important role in information-processing problems in adolescents with ASD, perhaps even more than the overall functioning of WM. The higher the information load in WM, as is the case with complex and rapidly changing social information, the more problems related to ASD emerge (Verté et al., 2006b).

Other possible, but ill-studied explanations for the different results of these WM studies may lie in the compensation strategies often applied by high-functioning adolescents with ASD in structured clinical or laboratory settings. By applying compensation strategies in these highly structured settings, these teenagers often show no deficits on relatively high-level tasks for assessing the constructs of theory of mind, weak central coherence and executive functioning (Rump et al., 2009; Salter et al., 2008), while in more complex or open-ended situations (for example, social interaction) these compensatory strategies may fail. An alternative account suggests that high-functioning individuals with ASD learn the 'rules' of social interaction by rote (declarative memory), although they lack the intuitive flexibility (non-declarative memory) by which children with normal development 'learn' and apply these social rules (Asperger, 1943; Hill \& Frith, 2003; Wing, 1997). This cognitive compensation strategy would demand more working memory involvement, which in turn would lead to a practice effect and a more efficient working memory network (Romero-Munguía, 2011). Recent neuroimaging research suggests that the transition from controlled to automatic working memory processing is associated with exponential signal decreases in task relevant regions. The temporal changes in brain activation patterns could be attributed to enhanced efficiency of information processing as a result of cognitive practice (Koch et al., 2006; Leon-Carrion et al., 2010). However, in these studies practice-related change in behavioural performance was found, for example, shorter mean response times and significantly more correct responses. This is in contrast with our findings and other studies (Crone \& Dahl, 2012). Moreover, this effect was found interindividually within one WM task. It is not clear how these findings would translate to other WM tasks and behaviour.

Thus, the complexity of the information to be processed and the stress this poses on WM seem to play a decisive role in whether or not spatial WM problems are found in high-functioning adolescents with ASD. This link between processing complex (social) information and WM is especially interesting; it gives an alternative explanation for the 'overwhelmed feeling' that individuals with ASD can get during complex information processing (Klinger, Klinger, \& Pohlig, 2007). 


\section{Neuroimaging studies}

Since the availability of non-invasive neuroimaging techniques, a stable and widely distributed network of cortical brain regions has been described, the fronto-parietal WM network, which is believed to be involved in WM processes in healthy adults (see Appendix-2). Until now, it has been proven to be very difficult to separate the temporal processing stages of WM and to allocate the various WM processes to different regions of the WM network. It remains unclear whether a distinction between functions cannot be found because of the inherent weakness of functional magnetic resonance imaging (fMRI) (for example, the low sensitivity and temporal resolution of the MRI BOLD signal), or because elementary WM processes are represented by a pattern of activity across the WM network. In other words, temporal processing may show the same network or different networks that are not detectable with fMRI. Nevertheless, there is a general belief that the prefrontal cortex acts as a control region in WM processes and, as one of its functions, filters out irrelevant items during the encoding of WM (Linden, 2007; McNab \& Klingberg, 2008; Takahama, Miyauchi, \& Saiki, 2010). Furthermore, the dorsolateral prefrontal cortex (DLPFC) is thought to play a role in creating and maintaining links between a subject's actions and their eventual

Table 2 Functional connectivity MRI studies on working memory in high-functioning adolescents with ASD

\begin{tabular}{|c|c|c|c|c|}
\hline $\begin{array}{l}\text { Study } \\
\text { [ref. number] }\end{array}$ & $\begin{array}{l}\text { Age yr } \\
\text { mean (SD) }\end{array}$ & $\begin{array}{l}\text { Participant } \\
\text { groups }\end{array}$ & $\begin{array}{l}\text { General } \\
\mathrm{IQ}(\mathrm{SD})\end{array}$ & $\begin{array}{l}\text { WM task } \\
\text { (differences task performance) }\end{array}$ \\
\hline \multirow{3}{*}{$\begin{array}{l}\text { Silk et. al. } \\
\text { (2006) [69] }\end{array}$} & $14.7(2.9)$ & $(n=7)$ Autism, AS & $114(16.9)$ & Mental Rotation task \\
\hline & \multirow[t]{2}{*}{$15.0(1.8)$} & \multirow[t]{2}{*}{$(n=9)$ TYP } & & (Accuracy: N) \\
\hline & & & & (Response time: N) \\
\hline \multirow{3}{*}{$\begin{array}{l}\text { Koshino et. al. } \\
(2008)[72]\end{array}$} & $24.5(10.2)$ & $(n=11)$ HFA & $104.5(13.1)$ & n-back (load $=0,1,2)$ \\
\hline & \multirow[t]{2}{*}{$28.7(10.9)$} & \multirow[t]{2}{*}{$(n=11)$ TYP } & \multirow[t]{2}{*}{$108.6(9.1)$} & (Accuracy: N) \\
\hline & & & & (Response time: N) \\
\hline \multirow{4}{*}{$\begin{array}{l}\text { Solomon et. al. } \\
\text { (2009) [71] }\end{array}$} & $15.2(1.7)$ & $(n=22) H F A, A S$ & $107(14)$ & POP task \\
\hline & \multirow[t]{3}{*}{$16.0(2.0)$} & \multirow[t]{3}{*}{$(n=23)$ TYP } & \multirow[t]{3}{*}{$113(11)$} & (Accuray low trials: N) \\
\hline & & & & (Accuray high trials: Y) \\
\hline & & & & (Response time: N) \\
\hline
\end{tabular}


outcomes in WM (Weissman et al., 2008). This allows previous experiences to guide the selection of future behaviours. Consistent with this view, a subject's ability to use feedback about previous actions to guide future behaviours can be severely impaired following damage to the DLPFC. This important role of the prefrontal cortex in WM processes fits the general assumption that WM and other executive functioning problems in autism are most likely the result of some form of prefrontal dysfunction (Hill, 2004; O'Hearn et al., 2008).

\section{Functional MRI}

As stated in Appendix-2, almost the same WM network is active in adolescents as in adults, albeit more refined in the latter. Thus, although the prefrontal cortex is still maturing during adolescence, it can be hypothesized that abnormal prefrontal functioning underlies WM problems in adolescents. However, only a few published fMRI studies have examined WM processing in individuals with ASD, and only one published study has been conducted in adolescents (Silk et al., 2006) (for an overview see Table 2). In that study, high-functioning adolescents with ASD had to perform a mental rotation task in a 3-tesla magnetic resonance imaging (MRI) scanner. When their cognitive results were compared with normal controls, there were no significant

\begin{tabular}{|c|c|}
\hline $\begin{array}{l}\text { Results } \\
\text { activation in ASD }\end{array}$ & connectivity in ASD \\
\hline$\downarrow$ anterior cingulate, $\downarrow$ DLPFC, & \multirow[t]{3}{*}{$\downarrow$ fronto-striatal } \\
\hline$\downarrow$ caudate nucleus, $\downarrow$ premotor cortex & \\
\hline$=$ posterior parietal cortex & \\
\hline$\downarrow$ inferior left prefrontal region, & \multirow[t]{3}{*}{$\downarrow$ frontal - parietal } \\
\hline no activation: right posterior temporal region & \\
\hline Shifted activation: fusiform face area & \\
\hline$=$ Low control trials & \multirow[t]{4}{*}{$\downarrow$ frontal - parietal } \\
\hline High control trials & \\
\hline$\downarrow$ anterior frontal region, $\downarrow$ parietal region, & \\
\hline$\downarrow$ occipital region & \\
\hline
\end{tabular}


differences between the ASD group and the comparison group on visuospatial task performance, response time or accuracy. Moreover, the neuroimaging results did not reveal any significant between-group differences in activation of the posterior parietal cortex. This area is thought to play a key role in mental rotation and is part of the dorsal visual processing stream that supports perception of spatial properties (Palmer, 2002). However, significantly less activation was found in the prefrontal area, including the anterior cingulate, dorsolateral prefrontal area, and the caudate nucleus, of the ASD participants: areas that play a significant role in the WM network in both adolescents and adults. Silk and colleagues (Silk et al., 2006) concluded that these results, despite the lack of significant differences in task performance between the two groups, may indicate a disruption of the network involved in WM and executive functions in high-functioning adolescents with ASD. These results should be interpreted with caution as the researchers did not look at the connectivity as such, and because this study was performed with a limited number of adolescents (seven adolescents with ASD and nine normal controls), especially since these results have not yet been replicated.

This disruption in the WM network was also suggested in another study performed in a larger cohort with high-functioning adolescents with ASD. This study used a WM-related cognitive task (the preparing-to-overcome prepotency or POP task), which is thought to measure cognitive control (Solomon et al., 2009). The POP task is designed to study cognitive control involved in context processing; for example, maintaining a cue over a delay and then overcoming a prepotent response tendency. In this study, reduced activation patterns in the frontal areas, and also in parietal and occipital regions, were found in the ASD group compared to controls. The correlation methods showed reduced functional connectivity between the frontal and parietal regions in the ASD group.

Aberrant functional connectivity patterns are also found in high-functioning young adults with ASD. In an n-back WM task for faces, Koshino and colleagues (Koshino et al., 2008) found a shift in activation patterns in the young adults with ASD compared with a normal control group. That is, the autism group showed less activation in the left frontal area, and no activation in the superior and middle gyri of the right posterior temporal lobes (associated with processing social information). In addition, the activation pattern in the fusiform face area was shifted towards a more lateral and inferior region. Functional connectivity results revealed that the fusiform activation in autism was less synchronized with frontal areas than in controls. Moreover, this activation pattern was not accompanied by activation in areas associated with social information processing. Despite these activation differences, cognitive performance was similar for both groups with high levels of accuracy and fast response times. 
Thus, these studies show barely any differences in task performance and fMRI results revealed no overall diminished brain activation during WM tasks in ASD adolescents. Yet, the patterns of activation in the fMRI studies were different from those of normal controls. This difference in activation patterns could indicate that adolescents with ASD use a different kind of WM network to achieve the same cognitive outcomes. This may represent a compensatory mechanism to preserve cognitive functioning (Silk et al., 2006). It is, however, unclear whether this compensatory strategy successfully works outside the structured laboratory setting in a more complex and demanding setting where WM load is even higher.

The reduced functional connectivity patterns of the WM network in adolescents with ASD seem to fit well within the cortical under connectivity theory proposed by Just and colleagues in 2004 (Just, Cherkassky, Keller, \& Minshew, 2004). This theory states that individuals with autism have a reduced functional connectivity throughout the brain, resulting in a deficit in integrating information at neural and cognitive levels. These deficits are most likely to arise when the tasks require integrative processing at a high level, regardless of the domain of the task. However, the results of the abovementioned studies may also be in agreement with other connectivity theories of autism, which propose hyper connectivity of local networks as opposed to global hypo connectivity (Casanova, 2004) or a combination of both hyper connectivity of local networks and hypo connectivity of global networks (Courchesne \& Pierce, 2005); for a review see (Belmonte et al., 2004).

\section{Structural MRI}

In contrast to the shortage of published research using fMRI and specifically studies on functional connectivity in adolescents with autism, much research has focused on the underlying structural brain abnormalities of autism. These structural MRI studies looked at a variety of brain characteristics such as cortical folding patterns, brain growth patterns, brain enlargement, brain volume, cortical thickness, and white-matter networks.

Earlier post-mortem and neuroimaging studies showed an abnormally high total brain volume and head circumference in young children with ASD; for a review see (Amaral, Schumann, \& Nordahl, 2008). These findings led to a theory of age-specific anatomic abnormalities in autism, first proposed by Courchesne and colleagues in 2001 (Courchesne, Campbell, \& Solso, 2011; Courchesne et al., 2001). This theory states that there are at least three definable periods of pathological brain development in ASD. Abnormal development starts with accelerated brain overgrowth soon after birth until young childhood. This period is followed by a period of abnormally slow brain growth between young childhood and older childhood/preadolescence. 
Subsequently, a third period starts in adolescence (and continues into young adulthood), which is marked by a premature and arrested growth of brain size; a period when the brain growth of non-ASD adolescents catches up with that of ASD adolescents.

In the last decade support for this theory has been found by a variety of volumetric MRI studies (Aylward, Minshew, Field, Sparks, \& Singh, 2002; Cheng, Chou, Fan, \& Lin, 2011; Courchesne et al., 2011; Freitag et al., 2009; Wallace, Dankner, Kenworthy, Giedd, \& Martin, 2010), which did not show abnormal total brain volumes in adolescents with ASD. These results may pinpoint adolescence as a time during which the cortical growth trajectories diverge a third time, which may affect the development of cognitive and functional milestones (Giedd, 2008; T. Paus, 2005; T Paus et al., 2008).

Table 3 Structural connectivity MRI studies on working memory in high-functioning adolescents with ASD

\begin{tabular}{|c|c|c|c|c|}
\hline $\begin{array}{l}\text { Study } \\
\text { [ref. number] }\end{array}$ & $\begin{array}{l}\text { Age yr } \\
\text { mean (SD) }\end{array}$ & $\begin{array}{l}\text { Participant } \\
\text { groups }\end{array}$ & $\begin{array}{l}\text { General } \\
\text { IQ (SD) }\end{array}$ & $\begin{array}{l}\text { Method } \\
\text { (differences in task performance) }\end{array}$ \\
\hline \multirow{2}{*}{$\begin{array}{l}\text { Barnea-Goraly et. al. } \\
\text { (2004) [91] }\end{array}$} & $14.6(3.4)$ & $(n=7)$ HFA & $101(12.2)$ & \multirow[t]{2}{*}{ Whole-brain voxel based analysis } \\
\hline & $13.4(2.8)$ & $(n=9)$ TYP & $107(8.5)$ & \\
\hline \multirow{2}{*}{$\begin{array}{l}\text { Cheng et. al. } \\
\text { (2010) [94] }\end{array}$} & $13.7(2.5)$ & $(n=25)$ ASD & $101.6(18.9)$ & \multirow[t]{2}{*}{ Whole-brain voxel based analysis } \\
\hline & $13.5(2.2)$ & $(n=25)$ TYP & $109.0(9.5)$ & \\
\hline \multirow{2}{*}{$\begin{array}{l}\text { Groen et. al. } \\
\text { (2011) [90] }\end{array}$} & $14.4(1.6)$ & $(n=17)$ ASD & $98(18)$ & \multirow[t]{2}{*}{ Whole-brain voxel based analysis } \\
\hline & $15.5(1.8)$ & $(n=25)$ TYP & $105(9)$ & \\
\hline \multirow{2}{*}{$\begin{array}{l}\text { Shukla et. al. } \\
\text { (2011) [92] }\end{array}$} & $12.6(0.6)$ & $(n=26)$ Autism, AS & $106.0(3.6)$ & \multirow[t]{2}{*}{ Whole-brain voxel based analysis } \\
\hline & $13.0(0.6)$ & $(n=24)$ TYP & $108.2(2.6)$ & \\
\hline \multirow{2}{*}{$\begin{array}{l}\text { Shukla et. al. } \\
\text { (2011) [93] }\end{array}$} & $12.6(0.6)$ & $(n=26)$ Autism, AS & $106.0(3.6)$ & \multirow[t]{2}{*}{ Whole-brain voxel based analysis } \\
\hline & $13.0(0.6)$ & $(n=24)$ TYP & $108.2(2.6)$ & \\
\hline
\end{tabular}

Note: $\mathrm{AS}=$ Asperger syndrome; $\mathrm{ASD}=$ Autism spectrum disorders; HFA= high-functioning autism; $\mathrm{NR}=$ not recorded; TYP = typical development. 
While no differences were found in total brain volume compared with typically developing teens, a variety of significant regional brain differences in adolescents with ASD were found: an enlarged prefrontal cortex, enlarged right inferior parietal cortex (Cheng et al., 2011), thinner left parietal and temporal cortices (Wallace et al., 2010), enlarged caudate nucleus (M. Langen, Durston, Staal, Palmen, \& van Engeland, 2007), and a reduced thickness of the splenium of the corpus callosum (Freitag et al., 2009). Other research showed increased folding in the frontal, parietal, and temporal lobes in both hemispheres and significant differences of cortical sulcal patterns in the superior frontal sulcus, Sylvian fissure, and inferior frontal, superior temporal and olfactory sulci in adolescents with autism (Awate, Win, Yushkevich, Schultz, \& Gee, 2008; Nordahl et al., 2007). Of these areas, several are part of the WM network and, as a consequence, these brain alterations could be related to the aberrant functional connectivity patterns found in ASD. However, it remains unclear what the clinical or cognitive significance of abnormal brain maturation in adolescents with ASD is.

\begin{tabular}{|l|l|l|}
\hline $\begin{array}{l}\text { Results } \\
\text { Location }\end{array}$ & FA & MD \\
\hline $\begin{array}{l}\text { Ventromedial prefrontal cortices, anterior } \\
\text { cingulate gyri, temporoperietal junctions }\end{array}$ & $\downarrow$ & NR \\
\hline right posterior limb of internal capsule & $\downarrow$ & NR \\
\hline frontal lobe, right cingulate gyrus, bilateral & $\uparrow$ & \\
\hline insula, right superior temporal gyrus, bilateral & & \\
\hline middle cerebellar peduncle & & \\
\hline cerebrum, cerebellum & $=$ & $\uparrow$ \\
\hline corpus callosum, anterior and posterior limbs & $\downarrow$ & $\uparrow$ \\
\hline internal capsule, inferior longitudinal-, inferior & & \\
\hline fronto-occipital-, superior longitudinal fasciculus & & \\
\hline cingulum, anterior thalamic radiation, & & \\
\hline corticospinal tract & & \\
\hline frontal lobe & $\downarrow$ & $\uparrow$ \\
\hline temporal lobes, parietal lobes & $=$ & $\uparrow$ \\
\hline
\end{tabular}




\section{Structural connectivity}

In general it is believed that abnormal functional connectivity does not necessarily imply abnormal structural connectivity, but deficient structural connectivity may underlie a lack of functional connectivity. In ASD, these structural connectivity patterns are increasingly being studied with diffusion tensor imaging, an MRI-based technique that measures the directional diffusion profile of water molecules, which reflects the axonal architecture of the brain at the micrometre level (Groen, Buitelaar, van der Gaag, \& Zwiers, 2011). With this technique, the various white-matter tracts in the human brain can be visualized and the structural integrity and connectivity can be revealed.

In high-functioning adolescents with ASD, widespread deficits in white-matter integrity have been reported frequently (for an overview see Table 3). One study reported reduced fractional anisotropy values adjacent to brain regions that have been implicated in social cognition (Barnea-Goraly et al., 2004), but most studies report more globalized deficits (Groen et al., 2011; Shukla, Keehn, \& Müller, 2011; Shukla, Keehn, Smylie, \& Müller, 2011). Only one study found the specific hyper- and hypo connectivity pattern proposed by Courchesne and colleagues, that is, an excessive disorganized and inadequately selective connectivity within the frontal lobe, and signs of hypo connectivity and poor synchronization between the frontal cortex and other brain regions (Cheng et al., 2010). However, another study states that hypo connectivity is global in autism, and that the short-distance tracts in the frontal, temporal, and parietal lobes are not selectively spared (Shukla, Keehn, Smylie, et al., 2011).

In short, although functional and behavioural studies strongly support a WM deficiency underlying the broad spectrum of problems high-functioning adolescents with ASD have, structural imaging studies point to a more global connectivity problem in the autistic brain. Abnormal growth patterns indicate a basis for the WM problems found in adolescents with ASD, but unfortunately, there are, to our knowledge, no studies that combine behavioural, functional, and structural imaging data from the same cohort of participants. Thus, no conclusions can be drawn with respect to the clinical implications of these findings.

\section{Conclusions}

Despite years of research and numerous studies, the cause and underlying mechanisms of ASD are still under debate. Finding a cause, or at least gaining more insight into the mechanisms behind this disorder, is still a key issue for understanding autism and for designing treatments. These findings are highly relevant, especially 
given the impact of this disorder on everyday functioning, and the increase in the number of individuals diagnosed with ASD.

Besides the well-known triad of problems, many people with ASD experience problems in executive functioning. Given the central role of executive functions in both higher and lower cognitive processes, problems in these functions could provide a good explanation for the symptoms seen in ASD. As WM is generally seen as a central process in many, if not all executive functions, we conclude that it is highly plausible that WM plays a leading role in the symptoms seen in ASD. To date, however, cognitive studies have failed to give conclusive evidence about the relationship between (in particular, spatial) WM functioning and the symptoms seen in high-functioning adolescents with ASD. WM has different components and one can argue that the mixed results were caused by the diversity of the tasks and the different WM mechanisms that were investigated. Nevertheless, in most studies even with different designs, WM problems increase when tasks impose heavier demands on WM. Thus the complexity of the information to be processed, rather than the specific content of the information to be processed, seems to play a decisive role in whether or not spatial WM problems are found in high-functioning adolescents with ASD.

Executive functions in general and WM processes in particular are generally linked to the functioning of the prefrontal cortex. This assumption is supported by functional MRI research, which shows a WM network containing parts of the prefrontal cortex and parietal cortex. In high-functioning adolescents with ASD, previous results seem to indicate a global WM processing or connectivity deficiency, instead of a more focused deficit limited to the prefrontal cortex. Atypical activation patterns found in both the posterior and prefrontal areas support this theory. Structural neuroimaging studies point to an even more global connectivity problem in an autistic brain.

$\mathrm{fMRI}$ also has limitations that should be taken in account. These limitations are mainly due to the complicated brain circuitry and functional networks that create neuronal mass activity as represented by the hemodynamic signal. This mass activity and the complicated differentiation between activation patterns imply that any results need to be interpreted with caution. Nonetheless, fMRI still represents an excellent tool, one that is currently available for gaining more insights into brain function; combining fMRI results with the results from behavioural studies should give better insights into the background, differences, and similarities of various psychological and psychiatric disorders. 
In our opinion, future studies should concentrate more on combining research methods. This may give researchers and clinicians more insight into the relationships between behaviour, cognition (inside and outside the laboratory), and the functional integrity of the brain. Moreover, studying behaviour, cognition, and the brain in the same cohort will make it easier to interpret fMRI results and the effect of (dys) functional neural networks on everyday living and potential compensation strategies. These insights may help researchers and clinicians to develop and use better intervention techniques for this group of important pervasive developmental disorders.

\section{Search strategy and selection criteria}

We searched PubMed for articles published between 1 January 2000, and 24 February 2012, with the terms 'working memory', 'adolescen*', and 'autism'. We included articles identified from these searches and relevant references cited in these articles. Only articles published in English were included. Studies on low-functioning individuals with autism were excluded.

\section{Appendix-1}

\section{Main cognitive theories of ASD}

Theory of mind deficit hypothesis (S Baron-Cohen et al., 1985): This refers to the ability to impute mental states to oneself and to others (Premack \& Woodruff, 1978), also called 'mentalizing.' This theory tries to explain the social and communicative difficulties of individuals with ASD. Earlier studies have suggested a developmental delay in theory of mind, independent of general intelligence (F. G. Happe, 1994b). High-functioning adolescents with ASD, however, often pass relatively high-level theory of mind tasks (Rump et al., 2009; Salter et al., 2008), which makes it hard to prove this theory for this population. Brain networks involved in mentalizing are: medial prefrontal cortex, temporoparietal junction, superior temporal sulcus, temporal poles and posterior cingulate cortex/precuneus. Additional, but less commonly activated regions: emotional networks (especially the amygdala), superior temporal lobes, and prefrontal cortex.

Weak central coherence account (Frith, 1989): This refers to a processing bias for featural and local information and a relative failure to extract gist or 'see the big picture' in everyday life. This theory explains symptoms of ASD that the theory of mind deficit hypothesis fails to explain: the repetitive interests and behaviour, obsessive desire for sameness, islets of ability, idiot savant abilities, excellent rote 
memory, and preoccupation with parts of objects. Recent research also showed that although high-functioning individuals with ASD have the tendency to process only local features of incoming information, they also can process this information more globally (Caron, Mottron, Berthiaume, \& Dawson, 2006; Plaisted, Dobler, Bell, \& Davis, 2006). This makes it hard to prove the weak central coherence theory in high-functioning individuals with ASD. And although this theory has been studied often, there is still a lack of specification and consensus at the neural and cognitive levels that underlie this theory.

Executive dysfunction theory (Hill, 2004): This refers to the executive function (EF) problems suffered by many individuals with ASD. Earlier studies showed clear behavioural problems in various executive function domains such as inhibition, planning, cognitive flexibility, verbal fluency, and visual working memory (Corbett et al., 2009; Geurts et al., 2004; Luna et al., 2002; S. Ozonoff et al., 1991; Robinson et al., 2009; Sinzig et al., 2008; Steele et al., 2007; Verté et al., 2006a, 2006b; Williams et al., 2006) in high-functioning individuals with ASD. This may explain the rigidity, repetitive interests, and the cognitive non-core symptoms of ASD. Although there is still no consensus on a specific neuropsychological profile of autism, and a few studies even failed to find any executive function problems in high-functioning individuals with ASD, most studies conclude that executive functions show a delayed developmental pattern from childhood to adolescence. Functional MRI data shows evidence for a delayed developmental trajectory for high-functioning children and adolescents with ASD, which may lead to a less sophisticated and flexible use of EF in adulthood (O'Hearn et al., 2008).

\section{Appendix- 2}

\section{Working memory networks in adolescence}

Functional imaging data shows that in adolescents and adults similar fronto-parietal WM networks are involved; cortical areas including the lateral premotor cortex, dorsal cingulate and medial premotor cortex, dorsolateral and ventrolateral prefrontal cortex, frontal poles, and medial and lateral posterior parietal cortex (Kaiser et al., 2010; McNab \& Klingberg, 2008; Osaka et al., 2004; Owen et al., 2005; Roth \& Courtney, 2007; A. Schaefer et al., 2006). However, as WM is still developing during adolescence there are also some differences. Adolescents, for example, recruit additional regions such as the hippocampus (Finn, Sheridan, Kam, Hinshaw, \& D'Esposito, 2010), or show BOLD intensity or pattern differences in the WM network while performing a WM task. Mostly these differences are explained by refinement and further specialization of the WM network during adolescence (Frangou, Chitins, \& Williams, 
2004; E. D. O'Hare, Lu, Houston, Bookheimer, \& Sowell, 2008; Olesen, Nagy, Westerberg, \& Klingberg, 2003; Vestergaard et al., 2010), likely due to the maturation processes of the brain: myelination, synaptic pruning, and synapse production and proliferation. Vestegaard and colleagues (Frangou et al., 2004; Olesen et al., 2003; Vestergaard et al., 2010) further suggested that there is a relation between white and grey matter maturation and WM performance in adolescents. 



\section{WORKING MEMORY NETWORK ALTERATIONS IN HIGH-FUNCTIONING ADOLESCENTS WITH AN AUTISM SPECTRUM DISORDER}

Submitted for publication as:

Barendse, E.M., Schreuder, L.J., Thoonen, G., Hendriks, M.P., Kessels, R.P., Backes, W.H., Aldenkamp, A.P., Jansen, J.F.

Working memory network alterations in high-functioning adolescents with an autism spectrum disorder. 


\section{Abstract}

Background: People with a disorder on the autism spectrum (ASD) typically have deficits in the working memory (WM) system. Working memory is found to be an essential chain in successfully navigating in the social world. We hypothesize that brain networks for WM have an altered network integrity in ASD compared to controls. Methods: 13 adolescents (1 female) with autism disorder $(n=1)$, Asperger syndrome $(n=7)$, and pervasive developmental disorder not otherwise specified $(n=5)$, and 13 typically developing control adolescents (1 female) participated in this study. Functional MRI was performed using an n-back task and in resting state.

Results: The analysis of the behavioural data revealed deficits in working memory performance in ASD, but only when tested to the limit. Adolescents with ASD showed lower binary global efficiency in the working memory network than the control group with n-back and resting state data. This correlated with diagnostic scores for total problems, reciprocity, and language.

Conclusion: High-functioning adolescents with ASD have difficulty with the working memory system, which they try to compensate for. Functional MRI markers of brain network organization in ASD are related to characteristics of ASD as represented in diagnostic scores. Therefore, functional MRI provides neuronal correlates for memory difficulties in high-functioning adolescents with ASD. 


\section{Introduction}

Autism Spectrum Disorder (ASD) is a neurodevelopmental disorder with an unknown brain aetiology. Consequently, the diagnosis is made based on behavioural assessments. ASD is characterized by persistent deficits in social communication and social interaction across multiple contexts, and restricted, repetitive patterns of behaviour, interests, or activities. These symptoms must be present in the early developmental period and cause clinically significant impairments in social, occupational, or other important areas of current functioning (Diagnostic and Statistical Manual of Mental Disorders -5 (DSM5)). Additionally, cognitive, and especially executive function problems, often co-occur (Hill, 2004; O'Hearn et al., 2008; S. Ozonoff et al., 1991; Robinson et al., 2009).

Executive functions are a group of higher cognitive functions necessary for the regulation of voluntary planned behaviour and the inhibition of task inappropriate responses (O'Hearn et al., 2008). Therefore, they are central to human cognition (Lezak et al., 2012). Working memory is a core component of these executive functions in its role as a temporary storage system under attentional control (Baddeley, 2012; Miyake et al., 2000). And, although working memory processes have always been related with abstract information processing, recent research shows that working memory is, in addition to the socio-affective processes, also essential for successfully navigating in the social world (Baddeley, 2012; Banko et al., 2009; E. Pellicano, 2010b; Perlstein, Elbert, \& Stenger, 2002). Accordingly, working memory deficits are often found in several developmental psychopathologies including ASD (for review see: (Barendse et al., 2013)).

It has been suggested that during adolescence, a complex fronto-parietal network of brain regions is involved in working memory processes (for review see: (Barendse et al., 2013)). Functional Magnetic resonance Imaging (fMRI) is a neuroimaging technique that allows the assessments of correlates of brain activity, by measuring changes in blood oxygenation levels that occur in response to the metabolic requirements of active neurons (van Veenendaal et al., 2015). In addition to the localization of which brain regions are involved in certain cognitive processes, fMRI also allows the assessment of the integrity of functional connections between brain regions. Using $\mathrm{fMRI}$, one can investigate the working memory network and assess whether it is abnormal in high-functioning adolescents with ASD, which might explain its impact on cognitive and social functioning.

We hypothesize that brain networks for working memory have altered network integrity outcomes in ASD compared to controls, possibly underlying social difficulties 
in adolescents with ASD. To investigate this, we compared a group of high-functioning adolescents with a normal developing control group using fMRI of a n-back working memory task and during resting state, in which we focused on activation patterns (n-back) and working memory network efficiency (n-back and resting state).

\section{Methods}

\section{Participants}

Fifteen participants with ASD were recruited from the special secondary education school 'de Berkenschutse' (located in Heeze, the Netherlands), as detailed previously (Drenthen et al., 2016). All high-functioning adolescents with ASD fulfilled established diagnostic criteria according to the DSM-IV, as well as the autism algorithm cut-offs on the Autism Diagnostic Observation Schedule (ADOS) (Lord et al., 2010). The control group consisted of eighteen normal developing adolescents. Control adolescents were recruited through advertisements in newspapers and most were living in different parts of the country.

Participants of both groups were between 12-18 years old. Adolescents in the control group were excluded if they and/or one of their siblings and/or parent(s) had a diagnosis ASD. Further exclusion criteria for both groups were 1) a comorbid psychiatric disorder, 2) a significant hearing or visual impairment, 3) an inability to speak/understand the Dutch language and/or 4) a comorbid central neurologic or other somatic disorder.

Two adolescents with ASD and five controls were excluded from the analysis; one because of a scanner malfunction and six because of a susceptibility artefact (braces) that could not be corrected for. Eventually 13 adolescents (1 female) with autism disorder $(n=1)$, Asperger syndrome $(n=7)$, and pervasive developmental disorder not otherwise specified $(n=5)$, and 13 normal developing control adolescents ( 1 female) participated in this study. Four adolescents with ASD used medication: three adolescents used methylphenidate, but discontinued medication at least 20-h before scanning allowing for complete washout. One adolescent with ASD used pipamperon (an antipsychotic drug) and did not discontinue medication. None of the control adolescents used medication. All adolescents had an estimated Full Scale IQ (FSIQ) over 105 [range ASD: 107-124; range controls: 107-135], as measured with a short version of the Wechsler Intelligence Scale for Children (WISC-III) (Campbell, 1998; Donders, 1997, 2001). The two groups did not differ from each other with respect to gender $(p=.567)$, age $(p=.102)$, Verbal Comprehension Index of the WISC-III $(p=.986)$, Freedom of Distractibility Index (FDI) $(p=.670), F S I Q(p=.179)$, and 
the ability to recognize emotions $(p=.414)$ as measured with the Emotion Recognition Task (Montagne et al., 2007) (Table 1).

The groups differed on working memory abilities in daily life as reported by parents on the Behavior Rating Inventory of Executive Function Working memory subscale $(p=.001)$, with parents of adolescents with ASD reporting more working memory problems in daily situations (Table 1).

The study protocol was approved by the Medical Ethical Commission of the Maastricht University Medical Centre. In accordance with the declaration of Helsinki, informed consents were obtained from all adolescents and their parents or caregivers.

Table 1 Clinical and demographic characteristics of the study groups

\begin{tabular}{|l|c|c|l|l|}
\hline & Control & ASD & & \\
\hline Gender (male/female) & M (sd) & M(sd) & Group comparison & $\eta_{p}{ }^{2}$ \\
\hline Age in months & $173.9(15.9)$ & $184.0(14.2)$ & $F(1,24)=2.892, p=.102$ & .108 \\
\hline Intelligence & & & & \\
\hline VCl & $117.2(10.5)$ & $117.1(9.0)$ & $F(1,24)=.002, p=.986$ & .000 \\
\hline POI & $105.5(8.1)$ & $114.5(9.2)$ & $F(1,40)=11.413, p=.002$ & .222 \\
\hline FDI & $103.9(14.0)$ & $98.9(11.7)$ & $F(1,40)=1.570, p=.218$ & .038 \\
\hline FSIQ & $113.2(7.8)$ & $116.7(5.0)$ & $F(1,24)=1.918, p=.179$ & .074 \\
\hline BRIEF Working memory & $14.0(3.3)$ & $19.2(3.8)$ & $F(1,23)=12.995, p=.001$ & .361 \\
\hline ERT & $58.0(9.9)$ & $57.7(5.1)$ & $F(1,22)=.693, p=.414$ & .031 \\
\hline CBCL & & & & \\
\hline Internalizing problems & $3.77(3.1)$ & $12.54(8.7)$ & $F(1,24)=11.630, p=.002$ & .326 \\
\hline Externalizing problems & $1.85(2.2)$ & $8.92(6.3)$ & $F(1,24)=14.614, p=.001$ & .378 \\
\hline Total problems & $10.46(7.7)$ & $43.31(22.3)$ & $F(1,24)=23.234, p=.000$ & .492 \\
\hline ADOS & & & & \\
\hline Communication & - & $3.15(1.9)$ & & \\
\hline RSI & - & $6.15(2.7)$ & & \\
\hline Total & - & $9.38(4.4)$ & & \\
\hline SBRI & - & $1.15(1.1)$ & & \\
\hline
\end{tabular}

Note: BRIEF = Behavior Rating Inventory of Executive Function; ERT = Emotion Recognition Task; RSI = Reciprocal Social Interaction; SBRI = Stereotyped Behaviors and Restricted Interests. 


\section{Experimental Paradigm}

During $\mathrm{fMRI}$, the adolescents performed a specific working memory task, the visual n-back task, with two experimental conditions: 1-back and 2-back. In the 1-back condition, participants were instructed to press the 'yes'-response button when the same picture (house or face) was presented twice in a row, if not they were instructed to press the 'no'-response button. In the 2-back condition they had to press the 'yes'-response button when the picture on the screen matched the one that had been represented two pictures ago, otherwise they had to press the 'no'-response button. The stimuli were 80 colour pictures of faces (male and female) from the database Radboud Faces Database (Langner et al., 2010) and 80 colour pictures of houses, presented with the E-Prime presentation software which also recorded the adolescents' behavioural performance. Each adolescent practiced for both experimental conditions in a separate practice session proximately 30 minutes before scanning.

The experiment in the scanner utilized a blocked design, with 4 epoch houses stimuli and 4 epoch faces stimuli for each of the experimental conditions (16 epochs total). Each epoch contained 15 pictures and 3, 4, 6, or 7 targets occurring at a random order per epoch. At the beginning of an experimental block, a visual instruction was given for $5500 \mathrm{~ms}$, followed by a fixation cross for $500 \mathrm{~ms}$. Each stimulus picture was shown for $1500 \mathrm{~ms}$, followed by a fixation cross for $500 \mathrm{~ms}$. After 15 stimuli, at the end of an epoch, the fixation cross was shown for $30.000 \mathrm{~ms}$ (see picture 1). The experimental conditions were presented in a fixed order, with first the 1-back condition followed by the 2-back condition. In between the two experimental conditions and after the 2-back task, a 7 minutes resting state MRI imaging took place. The total scanning time per experimental condition was 8 minutes, the total scanning time including the anatomic and resting state MRI scanning was 60 minutes.

Group comparisons on the behavioural data were made conducting GLM-MANCOVAs using the Statistical Package for Social Sciences version 20.0 for both experimental conditions separately. The dependent variable was the error rate as measured with the Total number of errors, Total number error negative ('no'-response when the correct response was 'yes'), Total number error positive ('yes'-response when the correct response was 'no'), and Total number error omission (no response). As previous research showed a relationship between intelligence level and working memory performance (Barbey et al., 2012; Friedman et al., 2006) we included FSIQ as a covariate in the analyses. 

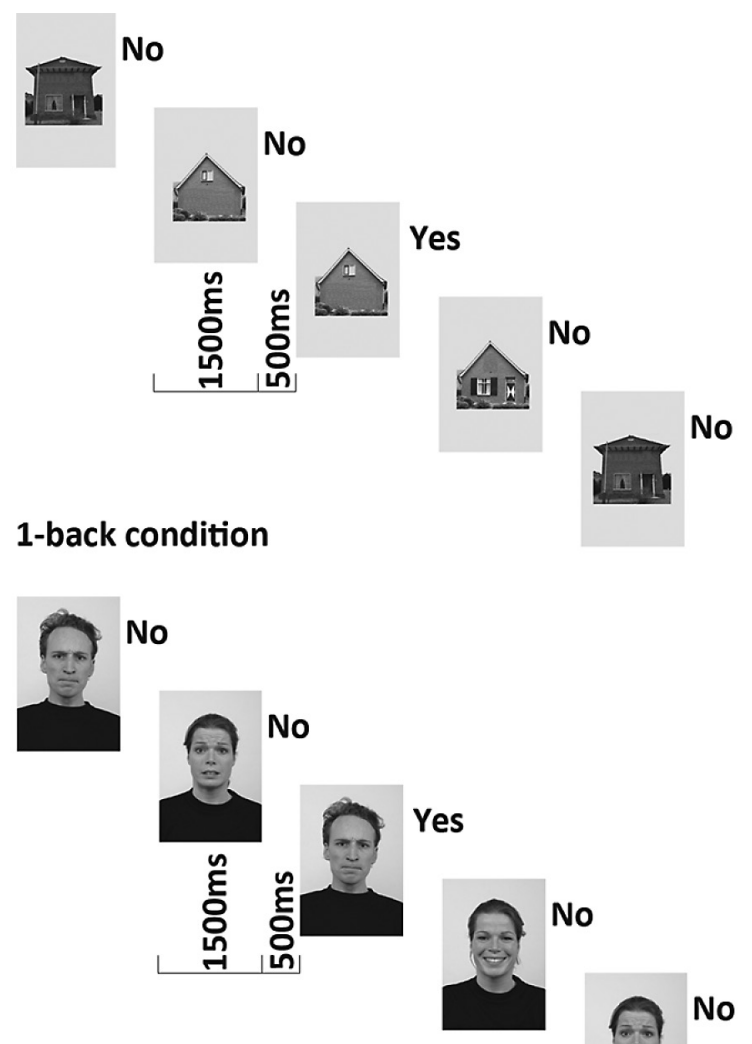

\section{2-back condition}

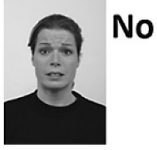

Figure 1 Schematic representation of the 1-back and 2-back condition. Each stimulus was presented for $1500 \mathrm{~ms}$ then followed by a fixation cross for $500 \mathrm{~ms}$. Stimuli of faces and houses were presented in both experimental conditions.

\section{Functional Imaging}

\section{fMRI parameters.}

fMRI data was acquired with a 3.0-Tesla unit (Achieva; Philips Medical Systems, Best, The Netherlands). An echo-planar imaging sequence is used with the following parameters: TR 2s, TE 35ms, Flip Angle 90 $0^{\circ}$, 31-32 slices, transverse orientation, slice thickness $4 \mathrm{~mm}$, in-plane resolution $2 \mathrm{~mm} \times 2 \mathrm{~mm}$, volumes per acquisition: 236 (n-back) and 210 (resting state). Additionally, for anatomical reference, a transverse orientated T1 weighted dataset was acquired with the following parameters: TR 8.233s, TE 3.77ms, Flip Angle $8^{\circ}, 150$ slices. 


\section{fMRI pre-processing}

The fMRI data was processed with Matlab R2012b (The MathWorks Inc., Natick, US) and SPM8 (Wellcome Trust Centre for Neuroimaging, UCL, London, UK). The functional images were realigned, resliced and slice time corrected. The mean image of the functional images was co-registered to the T1-weighted image. The T1-weighted image was segmented using Statistical Parametric Mapping v12. Functional images were normalized to the segmented image with a final voxel size of $2 \mathrm{~mm} \times 2 \mathrm{~mm} \times$ $2 \mathrm{~mm}$, and subsequently smoothed with a kernel of $8 \mathrm{~mm}$ full-width at half maximum. For n-back activation mapping: A GLM model was fitted that included two conditions: rest and picture assessment. Differences between the two conditions were assessed

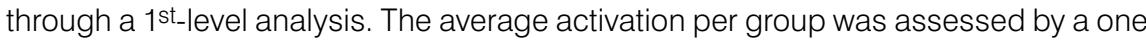
sample t-test with a family-wise error threshold of $p<0.05$. The differences between the two groups were assessed by a two sample t-test with a family-wise error threshold of $p<0.05$.

\section{Regions Of Interest definition}

Free Surfer (Martinos Center of Biomedical Imaging, Boston, US) software was used to segment the $\mathrm{T} 1$ weighted images of each subject into 84 cortical and subcortical regions. Additionally, a fronto-tempero subnetwork including 16 areas related to working memory was created, consisting of left and right lateral premotor cortex, dorsal cingulate and medial premotor cortex, dorsolateral and ventrolateral prefrontal cortex, frontal poles and lateral posterior parietal cortex and hippocampus (Fig. 2).

The Brodman areas found in research of Owen (Owen et al., 2005) were matched to their corresponding Freesurfer areas. The hippocampus was added, because research demonstrated that this is part of working memory of adolescents (Finn et al., 2010).

\section{Functional connectivity}

Pre-processed images of the resting state and the n-back tasks were realigned, co-registered to the atlas of the cortical and subcortical regions and smoothed with a kernel of $8 \mathrm{~mm}$ full-width at half maximum. The images were then band-pass filtered with a frequency window of $0.01-0.1 \mathrm{~Hz}$. To correct for physiological noise, linear regression with time series from the cerebrospinal fluid and white matter signals was applied. In addition, correction with linear regression for the moving parameters was applied. The sum of moving parameters in $x, y$ and $z$ direction was computed. No differences between the groups were found ( $p>0.22$ ) (resting state, $p=0.22$, mean ASD: 0.41 , mean controls: 0.35 ; 1 -back, $p=0.09$, mean ASD: 1.09 , mean controls: 0.50; 2-back, $p=0.29$, mean ASD: 1.67 , mean controls: 0.80 ). 


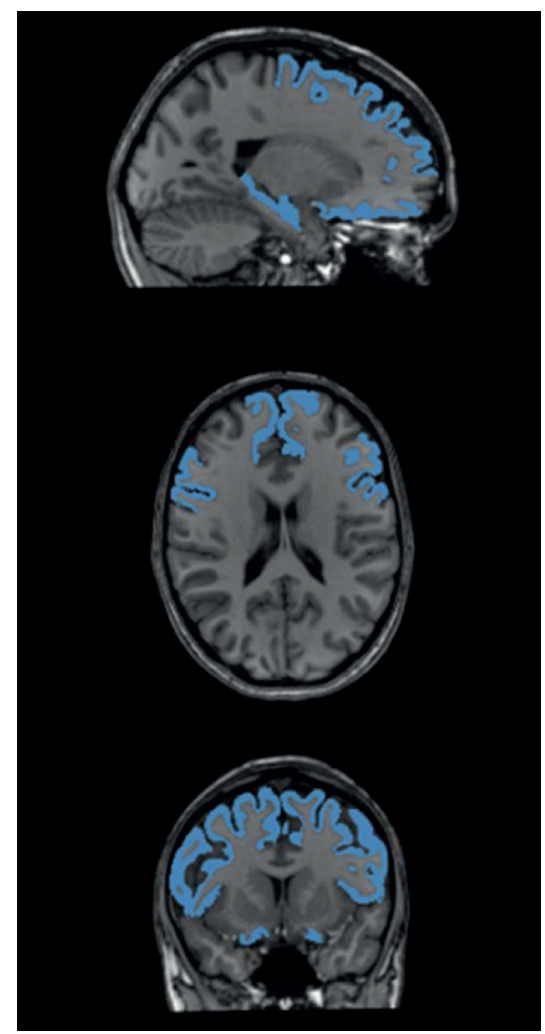

Figure 2 Selection of areas of the working memory network, including lateral premotor cortex, dorsal cingulate and medial premotor cortex, dorsolateral and ventrolateral prefrontal cortex, frontal poles and lateral posterior parietal cortex and hippocampus (all both left and right).

Pearson's linear correlation coefficients between the region-averaged time-series of all pairs of Free Surfer regions were computed. This resulted in an 84 x 84 connectivity matrix (whole brain) and a 16x16 connectivity matrix (working memory) for each subject. A Fisher's r-to-z transformation was applied at both connectivity matrices (Dunn \& Clark, 1969; Neill \& Dunn, 1975).

Negative correlations were set to zero (Van Dijk et al., 2010). Subsequently, a twosample t-test was applied with Bonferroni corrected p-value threshold for multiple comparisons. Additionally, permutation tests were performed to increase statistical power (Murray, Varnell, \& Blitstein, 2004). The subjects were randomly divided in two 
groups, which were repeated 1.000 times. Subsequently, for every connection the difference between the two groups was compared to the random realization using a t test.

Subsequently, the correlation matrix for each subject was made sparse. A percentage ranging from 20 up to $80 \%$ of connections with the highest correlation coefficients was included (Achard \& Bullmore, 2007; Vaessen et al., 2010).

Two different methods were applied to determine the sparse correlation matrices. In the first method, the average correlation matrix of all the subjects was used to create a sparse 'common' mask matrix, which was applied at each subject. This ensured that the sparse matrix for every subject contains the same connections. In the second method, the sparse matrix was determined for every subject separately, which yielded a unique sparse matrix on an individual level.

All connections greater than zero were set to 1 to create a binary matrix. Subsequently, with the Brain Connectivity Toolbox (Rubinov \& Sporns, 2010) four network metrics were calculated for the sparse connectivity matrices of each subject. We included the characteristic path length $(L)$, global efficiency $\left(E_{\text {global }}\right)$, modularity $(Q)$ and clustering coefficient $(\mathrm{C})$, both the binary as the weighted variants. A rank sum test was applied to investigate group differences using a p-value threshold of 0.05. The network metrics were correlated with Child Behavior Checklist Scores (CBCL, intern, extern, total), for the groups separately and for the whole group, and ADOS (communication, reciprocal social interactions and total) for the ASD group. To be able to determine whether the networks have small-world properties, the values for the characteristic path length and cluster coefficient were scaled to values from generated random networks. Random networks with small world properties are characterized by the characteristic pathlength close to random $\left(\lambda=\mathrm{L} / \mathrm{L}_{\text {random }} \approx 1\right)$ and the clustering coefficient higher than random $\left(\gamma=C / C_{\text {random }}>1\right)$. One hundred random networks were generated with the Brain Connectivity Toolbox.

\section{Results}

\section{Behavioural performance}

In the 1-back condition, the ASD and Control group showed similar behavioural performance, with high levels of accuracy. GLM- analysis revealed no significant main effects of group $[F(3,18)=.936, p=.444], F S I Q[F(3,18)=1.790, p=.185]$ or main interaction effect of group and $F S I Q[F(3,18)=.917, p=.452]$ on error rates. In the 2-back condition, main effects on the error rates of group $[F(3,19)=3.923, p=.025$, 
$\left.\eta_{\mathrm{p}}^{2}=.382\right], \mathrm{FSIQ}\left[\mathrm{F}(3,19)=4.449, \mathrm{p}=.016, \eta_{\mathrm{p}}^{2}=.413\right]$ and an interaction effect of group and $\mathrm{FSIQ}\left[\mathrm{F}(3,19)=3.814, p=.027, \eta_{p}^{2}=.376\right]$ were found. Subsequent ANOVAs showed a significant group effect on the Total number of error negative $\left[F(1,21)=8.194, p=.009, \eta_{p}^{2}=.281\right]$; Adolescents with ASD made more negative errors than controls on the 2-back task (Table 2).

Table 2 Behavioural data 1-back and 2-back condition

\begin{tabular}{|c|c|c|c|c|}
\hline & Control & ASD & \multicolumn{2}{|c|}{ group comparisson } \\
\hline \multicolumn{5}{|l|}{ 1-Back } \\
\hline \multirow[t]{2}{*}{ Gender (male/female) } & $11 / 1^{a}$ & $11 / 1^{a}$ & & \\
\hline & $M(s d)$ & $M(s d)$ & $p$ & $\eta_{p}^{2}$ \\
\hline Total no. errors & $6.33(3.4)$ & $4.75(3.7)$ & .842 & .002 \\
\hline Total no. errors negative & $2.08(1.9)$ & $1.58(2.1)$ & .285 & .057 \\
\hline Total no. errors positive & $3.75(2.1)$ & $2.75(2.0)$ & .334 & .047 \\
\hline Total no. errors omission & $.50(1.4)$ & $.42(1.4)$ & .762 & .005 \\
\hline \multicolumn{5}{|l|}{ 2-Back } \\
\hline \multirow[t]{2}{*}{ Gender (male/female) } & $11 / 1^{\mathrm{a}}$ & $12 / 1$ & & \\
\hline & $M(s d)$ & $M(s d)$ & $p$ & $\eta_{p}^{2}$ \\
\hline Total no. errors & $13.17(3.6)$ & $14.15(8.3)$ & .119 & .112 \\
\hline Total no. errors negative & $4.92(2.9)$ & $6.00(6.8)$ & .009 & .281 \\
\hline Total no. errors positive & $7.50(1.7)$ & $7.62(3.0)$ & .139 & .101 \\
\hline Total no. errors omission & $.75(.9)$ & $.54(1.1)$ & .415 & .032 \\
\hline
\end{tabular}

A significant interaction effect was found between group and FSIQ on Total number of errors negative $\left[F(1,21)=7.874, p=.011, \eta_{p}^{2}=.273\right]$ and subsequent Pearson correlation analysis per group revealed in the ASD group a significant negative correlation between FSIQ and Total number of negative errors $(r=-.664, p=.007)$. This significant relationship was not present in the Control group. 


\section{fMRI data analysis}

\section{Differences in activation.}

No significant differences in activation were found for the two groups.

\section{Functional connectivity.}

Resting state and n-back tasks data did not yield any significant differences in correlation on matrix element level, both for the whole as the WM network. Additionally, the permutation tests did also not yield any significant differences.

The individual method for the determination of the sparse matrices yielded the following results: the $E_{\text {global }}$ binary was significant in the sparsity range of 0.41-0.77 (resting state) (Fig. 3) and 0.59-0.7 (1-back) (Fig. 4)). After correction for random networks, $E_{\text {global }}$ binary showed a significant difference for sparsities from 0.5 up to 0.52 (resting state) and 0.55 up to 0.57 (1-back). Networks of the adolescents with ASD showed lower scores for $E_{\text {global. }}$.

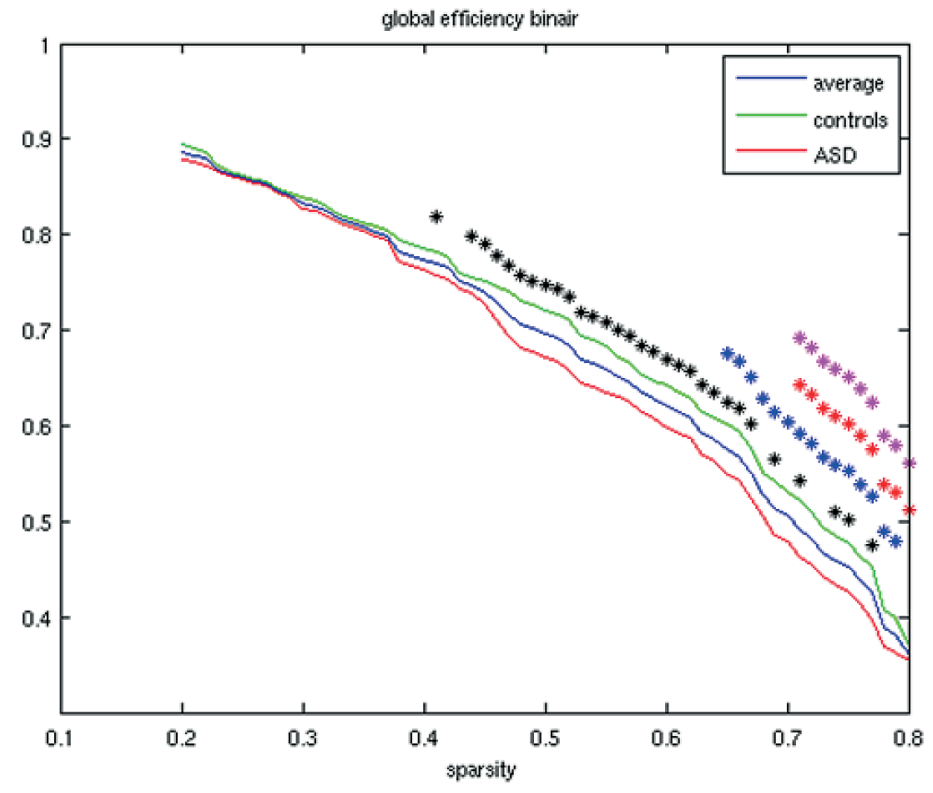

Figure 3 Adolescents with ASD showed lower binary global efficiency in the working memory network than the control group with resting state data. The black stars indicate a significant difference. Blue stars indicate significant negative correlations with $\mathrm{CBCL}$ total problems with the whole group, red stars' significant correlations with ADOS reciprocity and pink stars' significant correlations with ADOS total. 


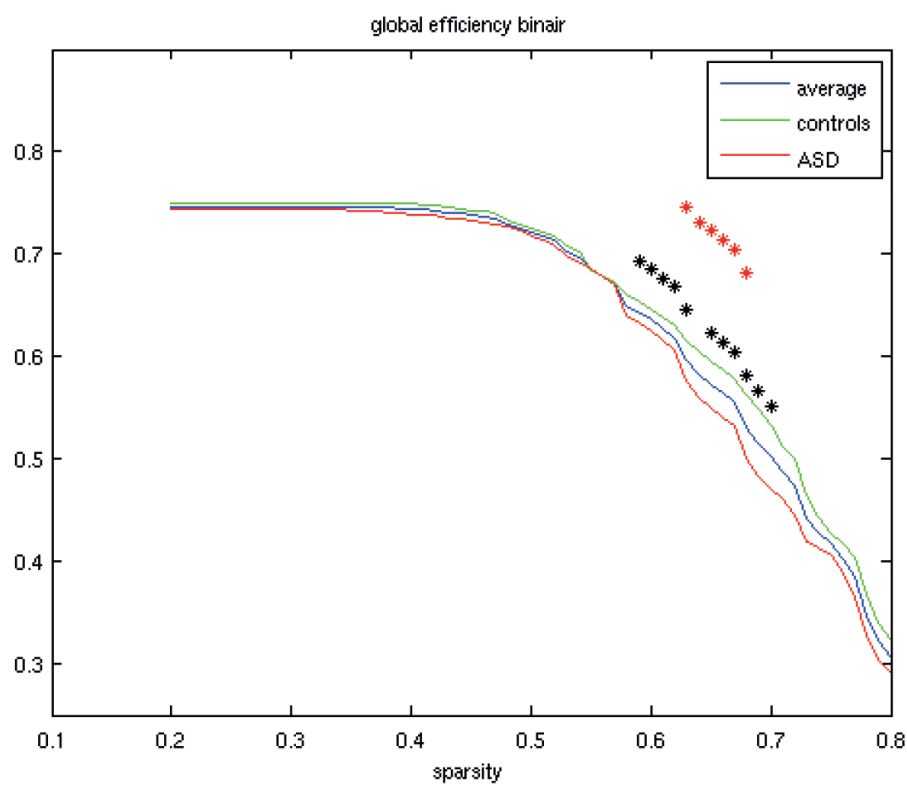

Figure 4 Adolescents with ASD showed lower binary global efficiency in the working memory network than the control group with 1-back data. The black stars indicate a significant difference. Red stars indicate significant correlations with ADOS language/communication.

The other network metrics did not show any significant difference. No significant results were found with the common mask method to calculate sparse matrices.

The global efficiency binary (resting state) correlated significant with $\mathrm{CBCL}$ total problems (sparsities 0.65-0.78) with correlations between -0.42 and -0.56 ( $\mathrm{p}$-values of 0.003-0.032), ADOS reciprocity (sparsities 0.71-0.8) with correlations between -0.62 and -0.76 ( $p$-values of $0.003-0.025$ ), ADOS total (sparsities) with correlations between -0.61 and -0.78 ( $p$-values of $0.002-0.028$ ).

The global efficiency binary (1-back) correlated significant with ADOS language/ communication (sparsities 0.63-0.69) with correlations between 0.55 and 0.65 (p-values of 0.027-0.049).

Network metrics corrected by the random networks yielded significant correlations for $\lambda$ (binary) in the sparsity range of 0.77-0.79 (resting state) and 0.55-0.57 (1-back), $\gamma$ (binary) in the range of $0.40-0.41$ (resting state)and $E_{\text {global }}$ (binary)for sparsities 
0.50-0.52 (resting state). For $\mathrm{E}_{\text {global }}$ binary overlapping sparsities were found for the uncorrected and corrected networks.

\section{Discussion}

The analysis of the behavioural data confirmed deficits in working memory performance in ASD, but only when tested to the limit (Hill, 2004). Statistically significant differences were not found on the 1-back task, but they did occur in the 2-back task. Adolescents with ASD made more negative errors than controls on the 2-back task. Moreover, in the ASD group a significant negative correlation was found between FSIQ and Total number of negative errors. This significant relationship was not present in the control group. The adolescents with ASD seem to rely more on their general intellectual capacities to solve more complex working memory tasks than typically developing adolescents, which is an indication that compensation occurs, likely related to the use of cognitive reserve capacity in these youngsters (Barendse et al., 2013).

In a brief report, Silk and colleagues found a dysfunction of the fronto-striatal network in high-functioning adolescents with ASD(Silk et al., 2006), which is in line with behavioural studies that reported executive and working memory problems in ASD. It has further been hypothesized that the functional connectivity between and within several neural networks is altered in individuals with ASD, due to immature or aberrant developmental processes(Rudie et al., 2012). Evidence for hypo and hyper connectivity have also been suggested in other $\mathrm{fMRI}$ studies that investigated working memory functioning in individuals with $A S D$, focused on broad age groups.

The two findings combined show a subtle neurological correlate showing weaknesses in WM processing that are largely compensating using cognitive reserve capacity. Thus, adolescents with ASD seem to rely more on their general intellectual capacities to solve more complex working memory tasks than typically developing adolescents. As anticipated, activation mapping revealed no differences between the groups. Pathology or changed brain function seldom leads to changed patters (localization, severity) of activated areas in reaction to a task (Vlooswijk et al., 2010). However, also resting state $\mathrm{fMRI}$ and n-back tasks data did not yield any significant differences in functional connectivity both for the whole as the specific WM network. This latter, much more sensitive, method was expected to yield differences in the organization of networks. 
The individual method for the determination of the sparse matrices yielded the following results:

1) Adolescents with $A S D$ showed lower binary global efficiency in the working memory network than the control group with resting state data. This correlated with $\mathrm{CBCL}$ (total problems) and ADOS (reciprocity and ADOS total) outcomes.

2) Adolescents with ASD showed lower binary global efficiency in the working memory network than the control group with 1-back data. This correlated with ADOS language/communication scores.

Global efficiency refers to the ability of a network to transmit information at the global level. An important metric that couples with network efficiency is 'network costs', that indicates how much effort is needed to maintain a network (Achard \& Bullmore, 2007). The loss of global efficiency suggest an increase of network costs, which may be a sign of the same compensatory process that is also seen in the behavioural data (Rane et al., 2015). Since the fMRI efficiency measures correlate with the behavioural data indicative of the severity of the disorder (lower efficiency is related to more social problems), compelling evidence is provided that links connectivity with the behavioural phenotype of the disorder.

We can therefore conclude that higher-functioning adolescent with an disorder on the autism spectrum have difficulty with the working memory system, which is typically compensated. Resting state fMRI and task related fMRI showed lower global efficiency parameters of brain network organization that were related to characteristics of ASD as represented in the CBCL and ADOS scores. Thus, fMRI provides a neuronal correlate of social deficits in high-functioning adolescents with an autism spectrum disorder. 

BRAIN RESTING-STATE NETWORKS IN HIGH-FUNCTIONING ADOLESCENTS WITH AN DISORDER ON THE AUTISM SPECTRUM: ANALYSIS OF SPATIAL CONNECTIVITY AND TEMPORAL NEURODYNAMICS

Submitted for publication as:

Bernas, A., Barendse, E.M., Aldenkamp, A.P. Backes, W.H., Hofman, P.A.,

Hendriks, M.P., Kessels, R.P., Willems, F.M., de With, P.H., Zinger, S., Jansen, J.F. Brain resting-state networks in adolescents with high-functioning autism: analysis of spatial connectivity and temporal neurodynamics. 


\section{Abstract}

Background: Autism Spectrum disorder (ASD) is mainly characterized by deficits in social communication and social interaction as well as restricted and repetitive behaviour. The leading hypothesis for the neural basis of ASD postulates globally abnormal brain connectivity, which can be assed using functional connectivity known as intrinsic, or resting-state connectivity. Global default connectivity in individuals with ASD versus controls is not well characterized, especially not for a high-functioning young population. The aim of this study is to test whether high-functioning adolescents with ASD have an abnormal resting-state functional connectivity.

Methods: We performed spatial and temporal analyses on Resting-State Networks (RSNs) in 13 high-functioning adolescents with ASD and $13 \mathrm{IQ}$ and age-matched controls. For the spatial analysis we used probabilistic Independent Component Analysis (ICA) and a permutation statistical method to reveal the RSN differences between the groups. For temporal analysis we applied Granger causality to find differences in temporal neurodynamics.

Results: Control adolescents and high-functioning ASD adolescents display very similar patterns and strengths of resting-state connectivity. We did not find significant differences between high-functioning adolescents with ASD and controls in the spatial resting-state connectivity. However, in the temporal dynamics of this connectivity we did find differences in the causal effect properties of RSns, originating in temporal and prefrontal cortices.

Conclusions: The results show a difference between high-functioning adolescents with ASD and normal developing control adolescents in the temporal neurodynamics from the ventral stream network to the executive network: a pathway involving in cognitive, executive and emotion-related cortices. We hypothesize that this weaker dynamic pathway is due to a subtle trigger challenging the cognitive state prior to the resting state. 


\section{Introduction}

Autism Spectrum Disorder (ASD) is a heterogeneous neurodevelopmental syndrome, which is characterized by persistent deficits in social communication and social interaction across multiple contexts and restricted, repetitive patterns of behaviour, interest or activities (DSM-5). Although not part of the diagnostic classification and not formal subcategories of ASD, a distinction is also often made between lowfunctioning autism (LFA) and high-functioning autism (HFA). No consensus criteria regarding LFA and HFA exist, but high functioning individuals with autism tend to have an 'normal' IQ (Barendse et al., 2013). Recently, increasing interest has been focused on abnormalities in (functional) brain organization in ASD, especially fMRI-based brain connectivity. Most reports in autism have focused on specific brain regions or networks related to cognitive functions such as working memory, executive function, visual attention and language processing (Anderson et al., 2011; Cherkassky, Kana, Keller, \& Just, 2006). Many task-based fMRI studies report that ASD is associated with either weaker or stronger connectivity between various structures (Monk et al., 2009). However, focusing on the 'resting-state' (i.e. task free) in fMRI provides a different domain to measure cortical synchronization patterns. Indeed, in the past decade, functional connectivity of resting-state fMRI data is rapidly emerging as a highly efficient and powerful tool for in vivo mapping of neural circuitry in the human brain (Zuo et al., 2010). Thus far, resting-state functional connectivity MRI studies in autism provide inconsistent results, i.e., showing either under- or hyperconnectivity in similar investigated brain regions (Muller et al., 2011; Uddin, Supekar, \& Menon, 2013).

To explore resting-state functional connectivity, neuroimaging researchers have adopted a multivariate signal processing method known as Independent Component Analysis (ICA). First, ICA is a multivariate, data-driven approach. It thus requires no a priori hypothesis about brain activity models (Beckmann, DeLuca, Devlin, \& Smith, 2005). Second, it detects interacting networks of regions, rather than just a single regiondominant (seed-ROI) network. Finally, this method is capable of automatically extracting noise such as scanner noise, physiological and motion artefacts (Thomas, Harshman, \& Menon, 2002). However, although ICA can provide spatial and temporal information about anatomical regions that show similar functional connectivity, it does not reveal causal relationships between components (the direction and effectiveness of the connectivity) (Deshpande, LaConte, James, Peltier, \& Hu, 2009; Liao et al., 2010). This causality, which we call 'temporal neurodynamics' in this paper, represents temporal causal effect dependencies between RSNs. 
Temporal neurodynamics of a brain can be visualized using the Wiener-Granger causality test (Bressler \& Seth, 2011) based on Granger's economical causality theory (Granger, 1969). This statistical test allows us to determine whether one-time series can be used to forecasting another. In other words, it is stated that a signal $X$ Granger-causes (or G-causes) a signal $Y$ if the past of $X$ does convey information about the future of $Y$ above and beyond all information contained in the past of $Y$ (Seth, 2010). Signals $X$ and $Y$, represent time series of co-activated neuronal populations which are, in our case, the temporal trends of the RSNs. Differences between high-functioning individuals with ASD and controls may be present in the composition of the spatial network organization (connectivity) or in the temporal neurodynamics (causal effect) or both.

\section{Methods}

\section{Participants}

Fifteen adolescents with ASD and 18 control adolescents participated in this study. Due to signal distortions caused by their braces, 2 participants with ASD and 5 control individuals were excluded from data analysis. All adolescents were between 12 and 18 years old. Individuals with ASD were recruited from De Berkenschutse, a special secondary education school in Heeze (the Netherlands). All adolescents in the control group were recruited through an advertisement in a (local)newspaper and visited regular secondary schools in various regions of the Netherlands. In accordance with the declaration of Helsinki, informed consents were obtained from all adolescents and their parents or caregivers. Inclusion criteria for the high-functioning adolescents with ASD were: established diagnostic criteria according to the DSM-IV, as well as the autism algorithm cut-offs on the Autism Diagnostic Observation Schedule (ADOS) (de Bildt et al., 2009). Inclusion criteria for the control group were: no history of psychological or psychiatric illness. Adolescents in the control group were excluded if they and/or one of their siblings and/or parent(s) had a diagnosis ASD. Further exclusion criteria for both groups were: a comorbid psychiatric disorder; a significant hearing or visual impairment; an inability to speak/understand the Dutch language; and/or a comorbid central neurologic or other somatic disorder. Table 1 shows the means and standard deviations of the ages in months and the Wechsler scores: The Verbal Comprehension Index (VCI), Perceptual Organization Index (POI), Freedom from Distractibility Index (FDI) and Full Scale Intelligence Quotient (FSIQ). Using the Multivariate Analysis of Variance (MANOVA) statistical method, we assessed the differences of the conditions (intelligence scores) of the both groups. The study protocol was approved by the Medical Ethical Commission of the Maastricht University Medical Centre. 
Table 1 Demographic and descriptive data of ASD and control adolescents

\begin{tabular}{|l|c|c|c|c|}
\hline & ASD & Controls & \\
\hline & M (sd) & M (sd) & $\begin{array}{c}\text { Group } \\
\text { comparison }\end{array}$ & $\mathbf{p}$ \\
\hline Gender (male/female) & $12 / 1$ & $12 / 1$ & & \\
\hline Age in years & $15.3(1.2)$ & $14.5(1.3)$ & $F(1,24)=2.89$ & 0.102 \\
\hline VCl & $117.1(9.0)$ & $117.0(10.4)$ & $F(1,24)=0.00$ & 0.986 \\
\hline POI & $114.0(5.8)$ & $109.1(7.8)$ & $F(1,24)=4.85$ & $\mathbf{0 . 0 3 8}$ \\
\hline FDI & $99.5(14.5)$ & $101.9(14.6)$ & $F(1,24)=0.19$ & 0.670 \\
\hline FSIQ & $116.7(5.0)$ & $113.2(7.8)$ & $F(1,24)=1.92$ & 0.179 \\
\hline
\end{tabular}

Note: $\mathrm{VCI}=$ Verbal Comprehension Index; $\mathrm{POI}=$ Perceptual Organization Index; FDI = Freedom from Distractibility Index; FSIQ = Full Scale Intelligence Quotient.

\section{Image acquisitions}

MRI was performed on a 3.0-Tesla unit (Philips Achieva) equipped with an 8-channel receiver-only head coil. For anatomical reference, a T1-weighted 3D fast (spoiled) gradient echo sequence was acquired with the following parameters: repetition time $8.2 \mathrm{~ms}$, echo time $3.7 \mathrm{~ms}$, inversion time $1022 \mathrm{~ms}$, flip angle $8^{\circ}$, voxel size $1 \times 1 \times 1 \mathrm{~mm} 3$, field of view 240x240 mm2, 150 transverse slices. Then, resting-state fMRI data was acquired using the whole brain single shot multi-slice BOLD echo-planar imaging sequence, with repetition time $2 \mathrm{~s}$, echo time $35 \mathrm{~ms}$, flip angle $90^{\circ}$, voxel size $2 \times 2 \times 4$ mm3, matrix 128x128, 32 contiguous transverse slices per volume, and 210 volumes per acquisition; resulting in total resting-state acquisition of 7 minutes. The resting-state scans were performed twice with an 8-minutes lasting 1-back test for working memory assessment in-between. This 1-back test was performed to assess the working memory processes. For this memory task, pictures of houses or faces (neutral and faces with one of the six standard emotion expressions) were displayed randomly at regular intervals. Then, ASD adolescents and controls were asked to indicate when the current stimulus (picture) matched the previous picture (Koshino et al., 2008). For the both resting-state scans, participants were instructed to lie with their eyes closed, to think of nothing but not to fall asleep.

\section{Data pre-processing}

Data analysis was carried out using FMRIB Software Library (FSL; www.fmrib.ox. ac.uk/fsl). The following pre-processing was applied (van der Kruijs et al., 2014): 
discard of the first 3 volumes ( $=6 \mathrm{~s}$ ) allowing the magnetization to reach equilibrium; rigid-body motion correction (Jenkinson, Bannister, Brady, \& Smith, 2002); non-brain tissue removal; slice-timing correction; registration to the Montreal Neurological Institute standard space (2 $\mathrm{mm}$ isotropic); spatial smoothing using a Gaussian kernel of $4.0 \mathrm{~mm}$ full-width at half-maximum; grand-mean intensity normalization; and high-pass temporal filtering at $100 \mathrm{~s}(0.01 \mathrm{~Hz})$. After these pre-processing steps one ASD participant and the second scan of a control participant were rejected because of a too large head motion: absolute displacement (mean) $>1 \mathrm{~mm}$ with a maximal relative displacement (between two consecutive slice) $>3 \mathrm{~mm}$.

\section{Group independent component analysis}

A single group-level ICA was performed across all subjects and all scans from both ASD and control group (total of $49 \mathrm{fMRI}$ acquisitions) using probabilistic ICA as implemented in FSL Multivariate Exploratory Linear Optimized Decomposition into Independent Components. First, the previously pre-processed 4D dataset was temporally transformed by concatenation into a single time series. This new 4D image was, then, separated into 34 independent components (ICs). The number of components (34) was manually optimized to provide optimal balance between the number of components required to capture the relevant RSNs while avoiding component splitting (separating each network into too many subnetworks) (Wang et al., 2011). To obtain the components, group probabilistic ICA processing steps were applied to the temporally concatenated 4D image: masking out non-brain voxels, voxel-wise demeaning of the data, and normalization of the voxel-wise variance. Subsequently, the pre-processed data were projected into a 34-dimensional subspace using probabilistic Principal Component Analysis. Then these observations were decomposed into sets of vectors which describe signal variations across the temporal domain (time-courses), the session/subject domain and the spatial domain (maps) by optimizing for non-Gaussian spatial source distributions using a fixed-point iteration technique (Hyvarinen, 1999). The resulting estimated component maps were divided by the standard deviation of the residual noise and threshold at a posteriori probability threshold of $p>0.5$ (i.e., an equal loss is placed on false positives and false negatives) by fitting a Gaussian/gamma mixture model to the histogram of intensity values (Beckmann et al., 2005).

\section{Resting state networks selection}

Eleven group-IC maps (out of 34) were selected according to the following 3 steps. First, group-IC maps with more than $33 \%$ of the estimated spectral power in high-frequencies $(>0.1 \mathrm{~Hz})$ were excluded to keep only networks within the low frequency range of 0.1-0.01 Hz (Lowe, Mock, \& Sorenson, 1998; Tyszka, Kennedy, Paul, \& Adolphs, 2014). Secondly, Smith and colleagues described the major co-varying 


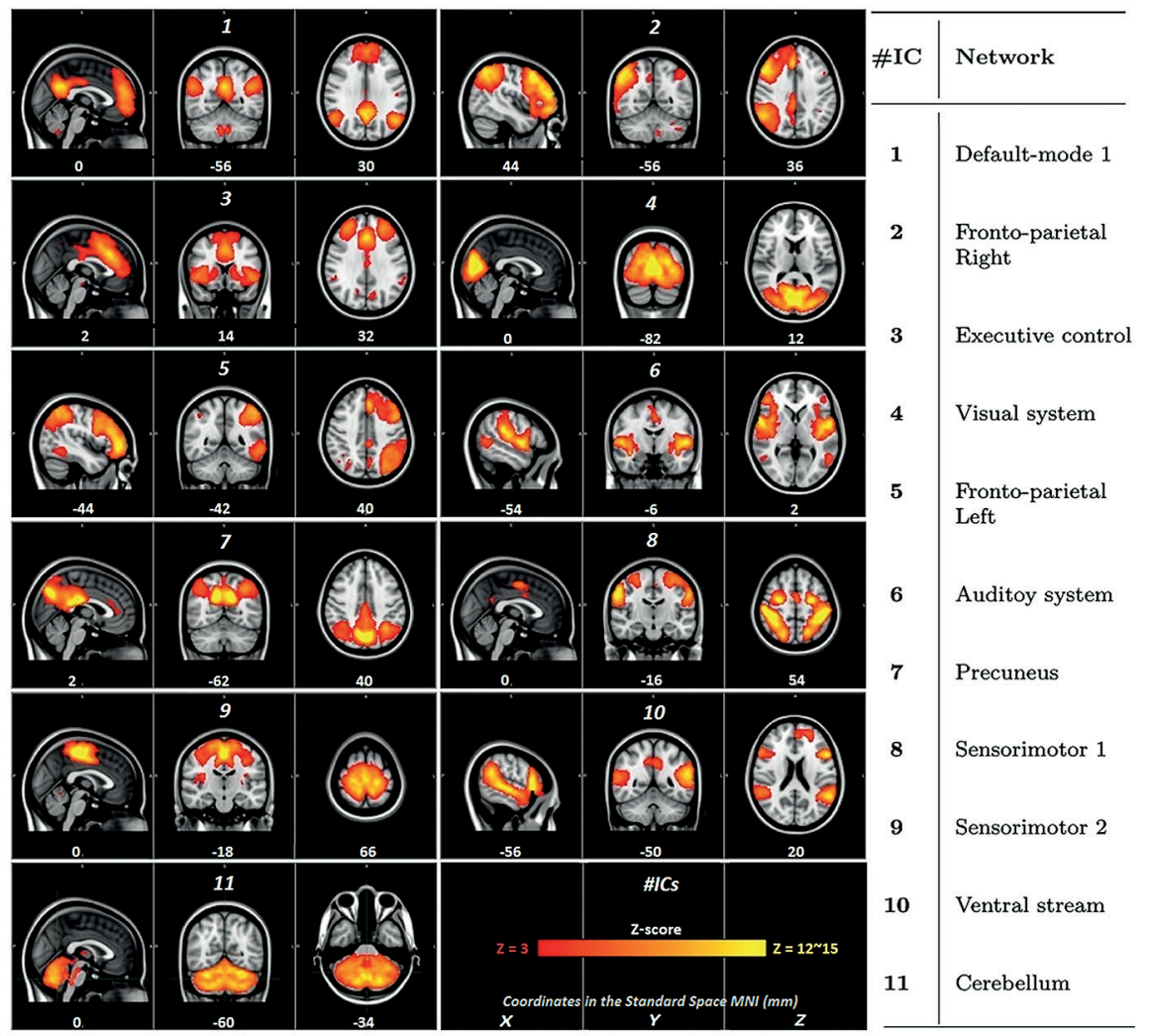

Figure 1 Relevant components extracted from the group-level ICA. Relevant components extracted from the 34 group-IC maps overlaid in colour on the Montreal Neurological Institute standard brain $(2 \times 2 \times 2 \mathrm{~mm})$. Names of the networks are in the right-side table. The threshold of the colour bar is between 3 and 15 (z-score). Montreal Neurological Institute coordinates are in $\mathrm{mm}$. The left hemisphere corresponds to the right side in the images (radiological convention).

networks in the resting brain and created a template of these RSNs widely used in resting-state $\mathrm{fMRI}$ studies (S. M. Smith et al., 2009). With this template and our remaining group maps, a function, using the 'goodness-of-fit' approach (Greicius, Srivastava, Reiss, \& Menon, 2004; Vanhaudenhuyse et al., 2010), was created and applied. Finally, the third step consisted in a visual inspection of each component's spatial profile to verify the consistency and ensure the effectiveness of the two previous steps. Figure 1 shows the 11 RSNs selected from the group-level ICA components. 


\section{Spatial RSN analysis between groups}

The first level of the voxel-wise group analysis was performed using dual regression (Filippini et al., 2009). The aim of this process is to obtain, from the group-IC maps, subject-specific IC maps. Dual regression involves two General Linear Models (GLM; Fig. 2). First, the group-IC maps were used as spatial regressors against the pre-processed individual fMRI scans. This results in single-subject time courses for each component separately (Fig. 2, block 1). Then, these time courses were normalized to unit variance to test both the "shape" and "amplitude" of the RSN. In the second GLM, these normalized individual time courses were used as temporal regressors against the pre-processed individual fMRI images. Subject-specific components were thus estimated (Fig. 2, block 2). We finally obtained subject-specific IC maps for each subject's scan. As there were two subject-specific spatial maps per IC for each individual (one per scan), before running final group-level analysis, we merged and averaged these two IC maps per subject. This resulted in 11 subjectspecific IC maps for each of the 25 participants.

The second level of the group analysis consisted in getting the effects of within-group means (control group average > 0; ASD group average $>0$ ) and between-group differences (ASD > control; control > ASD). This was assessed using non-parametric permutation testing (5000 permutations), with FSL's Randomize tool (Nichols \& Holmes, 2002). For each RSN, the resulting statistical maps were threshold at $p<0.05$ family-wise error corrected with the threshold-free cluster enhancer technique (S. M. Smith \& Nichols, 2009). Finally, nuisance regressors describing age, IQ and relative grey matter volume were added to the model in a second experiment, to observe their possible effects on the between-group contrast maps.

\section{Temporal dynamics of RSNs}

The statistical Granger causality allows us to assess causality among two signals. One signal $Y$ is said to Granger cause another signal $X$, if the past of $Y$ and $X$ can better predict the future of $X$ rather than just with the past of $X$ only (Zaremba \& Aste, 2014). In this study we use this principle to evaluate pairwise multivariate conditional Granger causalities of our independent components (resting-state networks). The assessment is made pairwise, which indicates that Granger causality will be performed on each pair of RSNs. It is also a directional calculation $\left(F_{Y \rightarrow X \mid Z} /=F_{Y \rightarrow X \mid Z}\right)$ (1), multivariate (multiple temporal signals) and conditional (G-causality depending on the past of the pair of signals and the additional information contributed by all the remaining signals). Suppose we have $X$ and $Y$, two temporal signals we want to assess their causality strength and $Z$ a third set of signals. The pairwise conditional Granger causality degree between these two signals (from Y to X) is defined by Geweke (1984) as follows: 

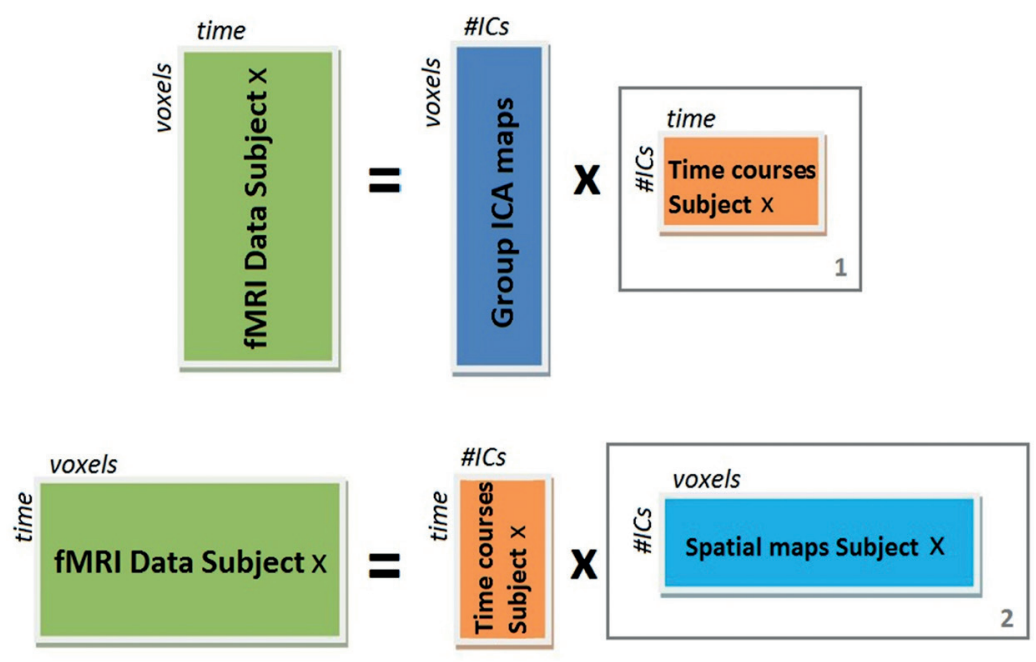

Figure 2 Dual-regression steps. Block 1 shows individual time course resulting from the first step (spatial regression). Block 2 shows the single-subject IC maps resulting from the second step (temporal regression).

$$
F_{y \rightarrow x} \equiv \ln \frac{\left|\sum^{\prime} x x\right|}{\left|\sum x x\right|}
$$

Where $\sum_{x x}^{\prime}$ is the variance of the prediction error (residuals) of $X$ conditional on $Z$ excluding the possible causal influence of $Y$, and $\sum_{x x}$ is the variance of the prediction error of $X$ conditional on $Z$, including also the possible causal influence of $Y$. Equation 1 can be expressed as "the degree to which the past of $Y$ helps predict $X$ over and above the degree of which $X$ is already predicted by its own past and the past of $Z$ " (Barnett \& Seth, 2014). By using the vector autoregressive model implemented in the Multivariate Granger Causality toolbox (Barnett \& Seth, 2014), we can measure the residuals $\left(\sum_{\mathrm{xx}}^{\prime}\right.$ and $\left.\sum_{\mathrm{xx}}\right)$ (eq. 1$)$ and hence, the G-causality magnitudes. Appendix-A describes the processing steps in more detail.

After the dual-regression is performed, we take the individual IC time courses for each scan/subject (See Fig. 2, block 1). With these temporal trends of the RSNs we estimate, with the Multivariate Granger Causality toolbox, the vector autoregressive model parameters. We obtain, thus, the conditional G-causality degree $F_{Y \rightarrow X \mid Z}$ (eq. 1) for each scan of each ASD adolescent and control adolescent, where $Y$ and $X$ are two RSN time series that we investigate directionally. In other words, we would like to 
answer the question: does network $Y$ 'G-cause' $X$ ? In our case, the G-causality is conditioned by all the other remaining RSN time series.

Finally, with 2-sample t-tests, we compare these G-causality magnitudes between the two groups (ASD vs controls) to determine different patterns of neuronal dynamics of the resting-state (effective connectivity). We also perform this test for the two resting-state scan sessions to assess whether or not, a previous task-based fMRI scan can trigger and/or change the 'classic state-of mind', i.e., its effective connectivity (neurodynamics between RSNs). And we finally assess if these changes differ between ASD adolescents and controls.

According to several studies, especially in task-based studies, individuals with ASD show differences mainly in frontal and temporal cortices, and also in resting-state brain connectivity within networks related to social interaction (Casanova, El-Baz, \& Suri, 2013; Wicker et al., 2008). Therefore, we selected the 4 RSNs located mainly in fronto-temporal cortices and/or consisting of socio-cognitive brain parts. These networks are compounded with the default mode network (IC 1, Fig. 1), the executive system (IC 3), the ventral stream (IC 10) and the auditory network (IC 6). These networks 200 are assessed and compared with the method described above.

\section{Results}

Intelligence scores are displayed in Table 1. Only the Perceptual Organization Index (POI) score showed a significant difference between the adolescents with ASD and the controls ( $p<0.05$, Tab. 1).

\section{Spatial RSN analysis between groups}

All the 11 networks were found in both ASD adolescents and controls by testing the subject-specific maps of these networks (after the dual regression). Figure 3 shows, in the relevant networks, the group effects (group mean $>0$ ) after a non-parametric permutation test (5000 permutations) with a threshold at $p<0.05$ threshold-free cluster enhancer corrected for family-wise error. These within-group inference maps showed a strong consistency with the whole group networks, when comparing Figures 1 and 3. No voxels, in any component, were significant in the second-level group analysis for the ASD > control and control > ASD contrasts. In the second equivalent final statistical analysis, where age, IQ level and grey matter density were added as covariates, again, no voxels in any component survived at the same threshold $(p<0.05$, family-wise error corrected; see Appendix-B Table B.2 for more 


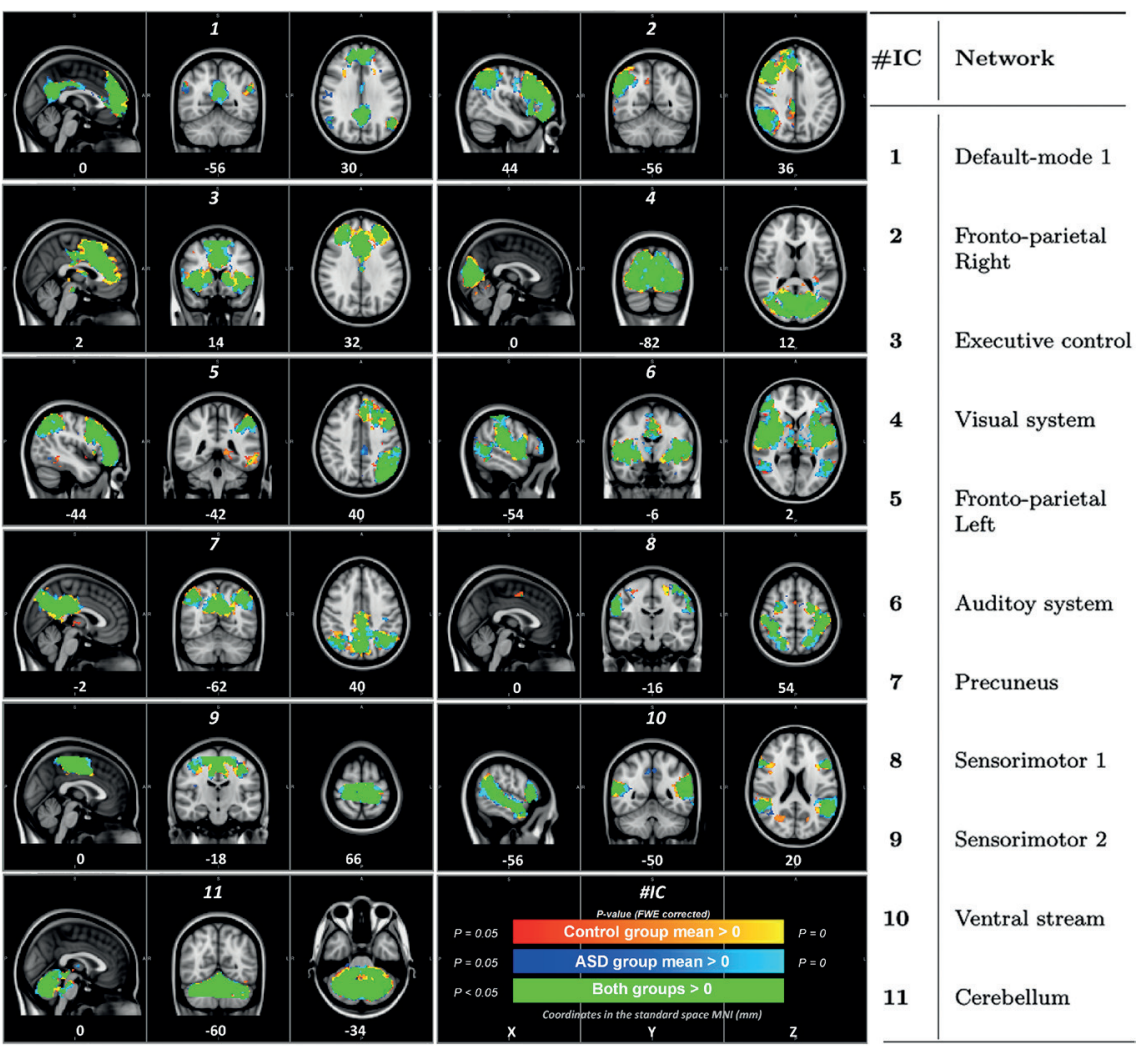

Figure 3 Group mean effect per network. Red-yellow = Control group; Blue-light blue = ASD group; Green $=$ Overlapping of both groups; with a threshold at $p<0.05$ family-wise error corrected.

details). Hence, statistically, the strength and the extent of each network (functional connectivity) was similar between both groups. 


\section{Temporal dynamics of the RSNs}

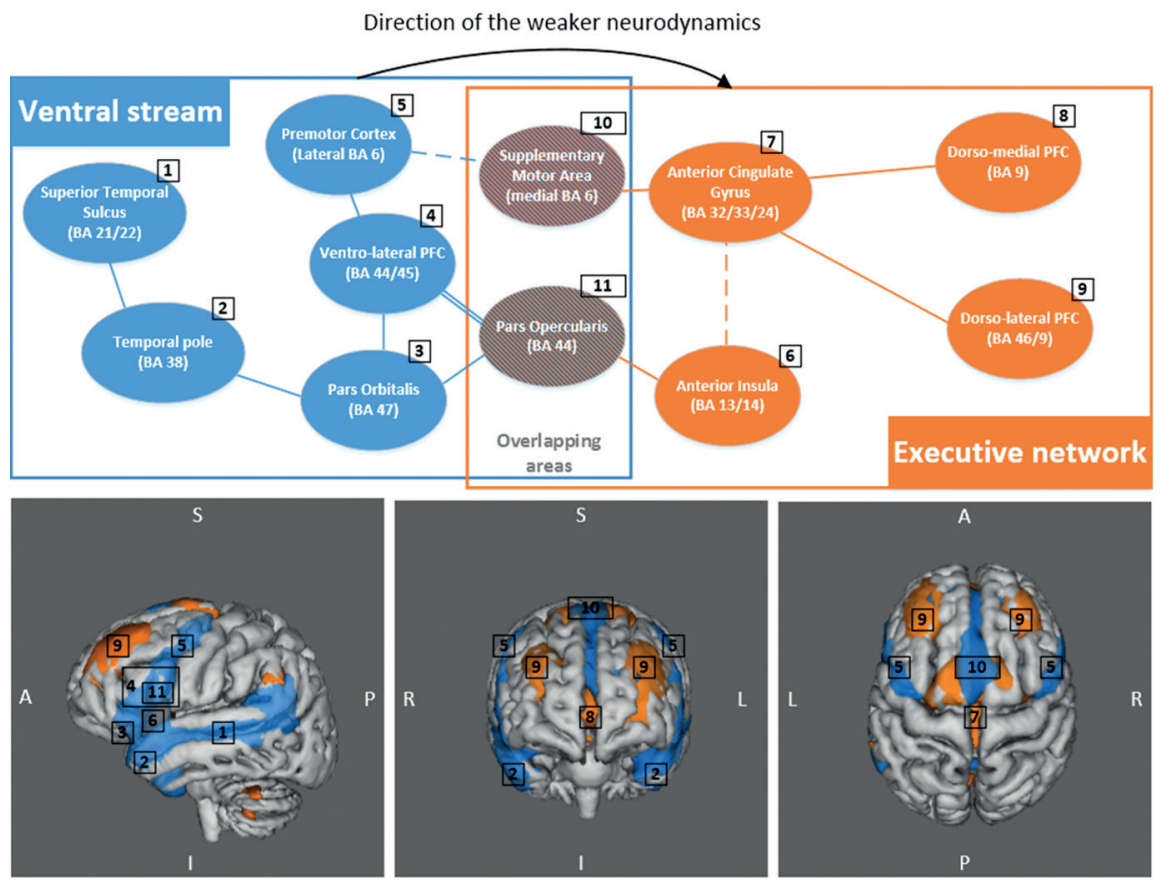

Figure 4 Visualisation of the weaker neurodynamic pattern in high-functioning adolescent with ASD. Visualisation of the two RSNs, executive in blue and ventral stream in orange, where the causal dynamics is weaker in high-functioning adolescents with ASD, in the direction from ventral to executive. The scheme above shows the different cortices involved in these two networks and the overlapping areas. Solid lines and dashed lines describe direct cortico-cortical physical link and indirect connections (through white matter and/or basal ganglia), respectively. The pars opercularis is a part of the ventro-lateral PFC which is shown with the double line. On the bottom part, anatomical visualization of the ventral stream and executive cortices (from group-IC contrast maps) are displayed (threshold at z-score $<2.6$, i.e., p-value $<0.01$ ). $\mathrm{BA}=$ Brodmann Area; PFC $=$ Prefrontal Cortex.

Pairwise conditional Granger-causality magnitudes, in average, within each group for the first and second resting-state scan sessions, were significant $(p<0.05$ FDR corrected). This was found for the 4 aforementioned selected prefrontal RSNs and for both groups. Also, the positivity of the normality test (Kolmogorov- Smirnov) for the distribution of the causalities among each group, allowed us to use the 2-sample t-test to compare ASD adolescents' G-causalities with those of the control group. 
In the first scan, none of the pairwise causalities differed significantly between ASD adolescents and controls. Same results were highlighted when comparing causalities within the control group (first scan vs second scan). However, dynamical RSN patterns did differ within the ASD cohort (first scan vs second scan) and significantly diverged from control adolescents only for the second resting-state scan. The latter describes a weaker dynamical (cause/effect) relation between the ventral stream and executive control networks, in the direction from ventral stream to executive network. Figure 4 shows this causal connection and displays in more detail the cortical regions involved in these two networks.

\section{Discussion}

In the present study of high-functioning adolescents with ASD compared with normal developing control adolescents, resting state whole-brain functional connectivity was examined. No evidence was found for any significant difference in brain spatial connectivity between the two populations. However, our results did show that patterns in temporal neurodynamics, i.e. causal effects of one RSN on another, differ between the groups. In contrast with controls, high-functioning adolescents with ASD display a significant difference in temporal neurodynamics between resting-state fMRI sessions 1 and 2. Furthermore, in contrast with the first resting-state scan, temporal neurodynamics differ significantly between high-functioning adolescents with ASD and normal developing controls during the second resting-state session. The primary findings of similar functional connectivity between the two cohorts challenge the theory that the autistic brain is globally under connected (Belmonte et al., 2004; Uddin et al., 2013). This can be explained by differences in scan protocols, in post processing methods (ICA vs seed-based) and mainly because of the population (type of the ASD, number and ages). However, our findings are in line with the results of Nomi and Uddin (2015) wherein, using also ICA, adolescents with ASD did not show any altered within-network functional connectivity. In their study, functional connectivity between the RSNs was also investigated by comparing pairwise correlations of RSN time series between the two cohorts. These pairwise temporal correlations can be seen as (undirected) instantaneous causality. Hence, their results of between-network hypo-connectivity in ASD population is partially (only instantaneous causality) in line with our results of weaker neurodynamics in ASD, which are discussed in the next paragraph.

To go further and detect strength and directionality in causality between RSNs, we used Granger causality upon the RSN time series. The two RSNs showing differences in effective directed connectivity (neurodynamics) are the ventral stream and the 
executive control network. The ventral stream contains mainly the left and right superior temporal sulci, the temporal poles, the ventro-lateral and orbital cortices, and lateral premotor cortex. This pathway is known to code for visual recognition and identification, and for emotional processes. The temporal pole is known to play a role in functions that tend to be weak in ASD: social and emotional processing, including face recognition and Theory of Mind (Olson, Plotzker, \& Ezzyat, 2007). Also, the superior temporal sulci has been postulated to be a critical component of the abnormal neural circuitry underlying deficits in social perception in ASD (Redcay, 2008). The superior temporal sulci projects information towards prefrontal cortices (mainly the medial and lateral) which are part of the executive RSN. This executive control network involves the anterior cingulate gyrus, the anterior insular cortex as well as the dorso-medial and dorso-lateral prefrontal cortices, and the supplementary motor area. These regions of interest are involved in cognitive processes such as working memory, reasoning, task flexibility, problem solving, planning and execution (Chan, Shum, Toulopoulou, \& Chen, 2008). The anterior insular cortex is a brain structure implicated in disparate cognitive, affective and regulatory functions, including interoceptive awareness, emotional responses, and empathic processes (Menon \& Uddin, 2010). More specifically, Daptretto and colleagues (Dapretto et al., 2006) propose a mirror neuron system dysfunction in children with ASD. Notably, they affirm that the mirror neuron system activity in the pars opercularis is consistently present during imitation, action observation, and intention understanding; and this pars opercularis combined with the insula and limbic activity (e.g., in the anterior cingulate gyrus) may mediate the understanding of others' emotional states. However, the absence of mirror neuron activity in the frontal part of the MSN (pars opercularis) leads this emotional process to be weaker in ASD. The association anterior insular cortex/ anterior cingulate gyrus (also termed the salient network) plays a role in dynamic switching between brain networks in reaction to cognitively demanding tasks (switch default mode network / executive network) (Menon \& Uddin, 2010). A review study reports that this critical system (salient network) is impaired in ASD, and that the anterior insular cortex region has demonstrated hypo-activity in individuals with ASD across a wide variety of social cognitive task paradigms (Uddin \& Menon, 2009).

All these findings illustrate that activations in brain areas implicated in the ventral and executive RSNs are known to be weaker in the ASD population. Also, areas in the salient network and the mirror neuron system, i.e., the causal flow 'bridge' area (Fig. 4), are critical during self- and other-related social and affective processes, and also known to be under-activated in ASD. In line with the previously mentioned studies conducted with the help of socio-emotional cognitive task-based fMRI, we observed the same weaknesses in whole brain resting-state functional connectivity, but only 
when analysing temporal dynamics. The extracted RSNs do not have the same pattern of temporal dynamics, i.e., the influence of one RSN on another varies between the two cohorts: the causal connectivity between the executive control network and the ventral stream (in the direction of ventral $\rightarrow$ executive) is significantly weaker in high-functioning adolescents with ASD. This impaired temporal neurodynamics suggests failing bridging of the emotional states regulated in the ventral stream to the decision-making oriented executive control system. This may therefore be described as a more rigid system in terms of the emotional-executive bridge.

In our study, the differences in temporal neurodynamics were only found in the second resting-state session. An n-back working memory task-based scan was performed in between the two resting-state scan sessions. We therefore hypothesize that abnormal temporal neurodynamic patterns in the group of high-functioning adolescents with ASD were triggered by the working memory task, involving not only working memory, but also attentional and emotional (in terms of face and emotion recognition) processes.

\section{Limitations}

One of the main challenges in applying G-causality of fMRI BOLD signals is the problem of the Hemodynamic Response Function changes. Interregional Hemodynamic Response Function variation has been argued to affect G-causality analysis (O. David et al., 2008). The Granger causality method implemented in Multivariate Granger Causality software as used in our study has been proven to be robust to changes in Hemodynamic Response Function properties (Seth, Chorley, \& Barnett, 2013). Also, the spectral density of our ICs being in the range of 0.01 and $0.1 \mathrm{~Hz}$, the HFR phenomenon (between 4 and 10 seconds) should not affect our temporal RSNs signals for the G-causality analysis. A second limitation with our technique is the relatively long sample intervals (repetition time) of classic fMRI protocols (usually ranging from $1 \mathrm{~s}$ to $3 \mathrm{~s}$ ). Indeed, our repetition time of $2 \mathrm{~s}$ is substantially longer than typical inter-neuron delays. However, since we examine changes (of differences) in G-causality rather than attempting to find a ground truth G-causality pattern, that limitation is not significant (Barnett \& Seth, 2014).

\section{Methodological recommendations}

For future application, we state that neurodynamics provide alternative strategies when ICA analysis does not yield differences for a cross-sectional analysis. Also, conversely, where ICA does show differences in functional connectivity between two populations (or more), we advise not to use Granger causality analysis on temporal trends of ICs, but rather on raw regions of interest signals (with the same locations for 
both groups). Finally, our findings show that tasks prior to resting-state acquisition scan have an effect on the results of an effective connectivity analysis.

\section{Conclusion}

We find no significant differences in resting-state brain connectivity between high-functioning adolescents with ASD and the control group at the whole brain level. However, the extracted RSNs do not have the same pattern of temporal dynamics, i.e., the influence of one RSN on another is different between the two cohorts. In particular, the causal connectivity between the executive control network and the ventral stream (in the direction of ventral $\rightarrow$ executive) is significantly weaker in the ASD group in the second resting-state scan, after challenging sensitive functions for the high-functioning adolescents with ASD. These two networks link cortices coding for face/object recognition and emotional processing with cortices of executive cognitive functions (attention, control, working-memory, behaviour). We hypothesize that changes in neurodynamics at rest in high-functioning adolescents with ASD are subtly triggered by challenging the cognitive state prior to the resting-state. These changes seem to appear in the networks functionally related to the previous cognitive task.

\section{Appendix-A}

\section{Multivariate Conditional Granger Causality theory}

We use a multivariate Granger causality MATLAB toolbox to calculate the conditional granger causality magnitudes. Algorithms and functions of this toolbox are described in Lionel Barnett's paper (Barnett \& Seth, 2014). The following parts describe the mathematical theory and processes needed behind Granger causality. Geweke (1984) describes the measure of the Granger causality as follows:

$$
F_{y \rightarrow x} \equiv \ln \frac{\left|\sum^{\prime} x x\right|}{\left|\sum x x\right|}
$$

In this equation, $\sum_{x x}^{\prime}$ is variance of the prediction error (residuals) of $X_{t}$ excluding the plausible influence $Y_{t}$, and $\sum_{x x}$ is variance of the prediction error of $X_{t}$ including the possible causal influence of $Y_{\mathrm{t}}$. To measure these variances, the vector autoregressive model (Deshpande, Santhanam, \& Hu, 2011; Roebroeck, Formisano, \& Goebel, 2005; Zhou et al., 2011) is applied. This model is defined as:

$$
U_{t}=\sum_{k=1}^{p} A_{k} \cdot U_{t-k}+\varepsilon_{t}
$$


Here, $U_{t}$ is a time series, $A_{k}$ - regression coefficients, $\varepsilon$ - the residuals and $p$ - the model order representing the number of past lags taken into account. This model order is calculated from time series using the Akaike Information Criterion (Akaike, 1974; Deshpande et al., 2009). If $U_{t}$ is a multivariate vector composed if two time series $X$ and $Y$, the vector autoregressive model can be developed as shown in Equation A.4:

$$
\begin{aligned}
& U_{t}=\left(\begin{array}{l}
X_{t} \\
Y_{t}
\end{array}\right) \\
& \left(\begin{array}{l}
X_{t} \\
Y_{t}
\end{array}\right)=\sum_{k=1}^{p}\left(\begin{array}{ll}
A_{x x, k} & A_{x y, k} \\
A_{y x, k} & A_{y y, k}
\end{array}\right)\left(\begin{array}{l}
X_{t-k} \\
Y_{t-k}
\end{array}\right)+\left(\begin{array}{c}
\varepsilon_{X, t} \\
\varepsilon_{y, t}
\end{array}\right)
\end{aligned}
$$

With the covariate matrix of the residuals expressed as:

$$
\sum \equiv \operatorname{cov}\left(\begin{array}{l}
\varepsilon_{x, t} \\
\varepsilon_{y, t}
\end{array}\right)=\left(\begin{array}{l}
\sum x x \sum x y \\
\sum y x \sum y y
\end{array}\right)
$$

By applying the Morf's variant of the Locally Weighted Regression algorithm (Morf, Vieira, Lee, \& Keilath, 1978), which is very efficient for the likelihood-based model selection such as the Akaike Information Criterion (McQuarrie \& Tsai, 1998), we estimate the vector autoregressive parameters. If we consider the $X$ - component of the regression (Eq. A.4), we obtain the following expression:

$$
X_{t}=\sum_{k=1}^{p} A_{x x, k} \cdot X_{t-k}+\sum_{k=1}^{p} A_{x y, k} \cdot Y_{t-k}+\varepsilon_{x, t}
$$

And if we suppose no conditional dependence between $X$ and the past of $Y\left(A_{x y, 1}=\right.$ $A_{x y, 2}=\ldots=A_{x y, p}=0$ ), we obtain the reduced regression:

$$
X_{t}=\sum_{k=1}^{p} A_{x x, k}^{\prime} \cdot X_{t-k}+\varepsilon_{x, t}^{\prime}
$$

We finally get from Equation A.5 the following: $\sum_{x x}=\operatorname{cov}\left(\varepsilon_{x, t}\right)$ and $\sum_{x x}^{\prime}=\operatorname{cov}\left(\varepsilon_{x . t}^{\prime}\right)$. Hence, we get the Geweke measure of the Granger causality (Eq. A.1).

In our case, we have more than two time series, thus Granger causality between one signal and another is depending on the information added by the past of the other signals. Let us now illustrate this conditional pairwise G-causality. Suppose we have:

$$
U_{t}=\left(\begin{array}{l}
X_{t} \\
Y_{t} \\
Z_{t}
\end{array}\right) \text {, }
$$


Where $Z_{t}$ is a third set of variables. We aim at eliminating any joint effect of $Z$ on the inference of the G-causality $Y$ to $X$. Thus, analogously to Equation A.4, the full and reduced regression for the $X$ - component becomes respectively:

$$
X_{t}=\sum_{k=1}^{p} A_{x x, k} \cdot X_{t-k}+\sum_{k=1}^{p} A_{x y, k} \cdot Y_{t-k}+\sum_{k=1}^{p} A_{x z, k} \cdot Z_{t-k}+\varepsilon_{x, t}
$$

And

$$
X_{t}=\sum_{k=1}^{p} A_{x x, k}^{\prime} \cdot X_{t-k}+\sum_{k=1}^{p} A_{x z, k}^{\prime} \cdot Z_{t-k}+\mathcal{E}_{x, t}^{\prime}
$$

Which corresponds to Equation A.6 and Equation A.7, but with the inclusion of condition variables $Z$ in both regressions. We can finally quantify the conditional G-causality as the log-likelihood ratio:

$$
F_{y \rightarrow x \mid z} \equiv \ln \frac{\left|\sum^{\prime} x x\right|}{\left|\sum x x\right|}
$$

Which can be defined as "the degree to which the past of $Y$ helps predict $X$ over and above the degree of which Z" (Barnett \& Seth, 2014).

\section{Appendix-B}

Table B.2 FEW-corrected p-values of the first voxels $(\geq 1)$

\begin{tabular}{|l|c|c|c|c|}
\hline Network & \multicolumn{3}{|c}{ p-value (corrected) } \\
\hline Con> ASD & ASD > Con & Con> ASD adj* & ASD > Con adj* \\
\hline Default-mode 1 & 0.93 & 0.54 & 0.85 & 0.58 \\
\hline Fronto-parietal R & 0.94 & 0.13 & 0.59 & 0.23 \\
\hline Executive control & 0.39 & 0.36 & 0.65 & 0.66 \\
\hline Visual system & 0.33 & 0.83 & 0.38 & 0.74 \\
\hline Fronto-parietal L & 0.29 & 0.60 & 0.10 & 0.38 \\
\hline Auditory system & 0.97 & 0.59 & 0.79 & 0.61 \\
\hline Precuneus & 0.39 & 0.94 & 0.13 & 0.82 \\
\hline Sensorimotor 1 & 0.20 & 0.71 & 0.60 & 0.43
\end{tabular}





\section{ALTERED NEUROTRANSMITTER METABOLISM IN ADOLESCENTS WITH HIGH-FUNCTIONING AUTISM}

\section{Published as:}

Drenthen, G.S., Barendse, E.M., Aldenkamp, A.P., van Veenendaal, T.M., Puts, N.A., Edden, R.A., Zinger, S., Thoonen, G., Hendriks, M.P., Kessels, R.P., \& Jansen, J.F. (2016).

Altered neurotransmitter metabolism in adolescents with high-functioning autism. Psychiatry Research, 256, 44-49. doi: 10.1016/j.pscychresns.2016.09.007. 


\section{Abstract}

Background: Previous studies have suggested that alterations in excitatory/inhibitory neurotransmitters might play a crucial role in autism spectrum disorder (ASD). Proton magnetic resonance spectroscopy (1H-MRS) can provide valuable information about abnormal brain metabolism and neurotransmitter concentrations. However, few $1 \mathrm{H}-\mathrm{MRS}$ studies have been published on the imbalance of the two most abundant neurotransmitters in ASD: glutamate (Glu) and gamma-aminobutyric acid (GABA). Moreover, to our knowledge none of these published studies is performed with a study population consisting purely of high-functioning adolescents with ASD. Selecting only high-functioning individuals eliminates factors possibly related to intellectual impairment instead of ASD. This study aims to assess Glu and GABA neurotransmitter concentrations in high-functioning adolescents with ASD.

Methods: Occipital concentrations of Glu and GABA plus macromolecules (GABA+) were obtained using $1 \mathrm{H}-\mathrm{MRS}$ relative to creatine $(\mathrm{Cr})$ in high-functioning adolescents with ASD ( $n=15$ and $n=13$ respectively) and a normal developing control group ( $n$ $=17$ ).

Results: Multiple linear regression revealed significantly higher $\mathrm{Glu} / \mathrm{Cr}$ and lower GABA+/Glu concentrations in the ASD group compared to the controls.

Conclusion: The results imply that imbalanced neurotransmitter levels of excitation and inhibition are associated with high-functioning adolescents with ASD. 


\section{Introduction}

Autism spectrum disorder (ASD) is a pervasive neurodevelopmental disorder, which is characterized by deficits in social interaction, impaired verbal and non-verbal communication, and restricted and repetitive behavior (Diagnostic and Statistical Manual of Mental Disorders, fourth ed., 1994). The neural mechanisms of ASD are not yet completely understood, although there is evidence that ASD is associated with abnormal brain development (Barendse et al., 2013; Baruth, Wall, Patterson, \& Port, 2013; Rump et al., 2009; G. B. Schaefer \& Lutz, 2006). Furthermore, several previous post-mortem studies (Fatemi, Reutiman, Folsom, \& Thuras, 2009; Zikopoulos \& Barbas, 2013) and electroencephalography studies (Orekhova et al., 2008; Rubenstein \& Merzenich, 2003; Snijders, Milivojevic, \& Kemner, 2013) have suggested impairments in the regulation of the inhibitory/excitatory system of individuals with ASD. To study the inhibitory/excitatory system a magnetic resonance technique can be used that quantifies the primary excitatory and inhibitory neurotransmitters.

Proton magnetic resonance spectroscopy (1H-MRS) allows for a non-invasive in vivo measurement of metabolite concentrations in a specific part of the brain. Using $1 \mathrm{H}-\mathrm{MRS}$, the radio-frequency signals that arise from nuclear spins can be detected. The resonant frequencies of these signals depend on their chemical environment and are referred to as chemical shift in parts per million of the proton frequency (Puts \& Edden, 2012). Since the corresponding chemical shifts for most metabolites are known, the $1 \mathrm{H}-\mathrm{MRS}$ spectrum can be analyzed to yield metabolite concentrations. Therefore, it is possible to study potential abnormalities in brain neurochemistry using $1 \mathrm{H}-\mathrm{MRS}$. In this study, $1 \mathrm{H}-\mathrm{MRS}$ is used to quantify the concentrations of neurotransmitters glutamate (Glu), the primary excitatory neurotransmitter (Simpkins \& Simpkins, 2013) and gamma-aminobutyric acid (GABA), the primary inhibitory neurotransmitter (Lainhart, Cooperrider, \& Taylor, 2013).

Several invasive studies have already demonstrated different levels of Glu and GABA in the autistic brain. In these studies increased levels of GABA (Dhossche et al., 2002; El-Ansary \& Al-Ayadhi, 2014) and Glu (Aldred, Moore, Fitzgerald, \& Waring, 2003; El-Ansary \& Al-Ayadhi, 2014; Tu, Chen, \& He, 2012) and decreased levels of the Glu/ GABA ratio (El-Ansary \& Al-Ayadhi, 2014) were reported in blood plasma. Moreover, previous non-invasive $1 \mathrm{H}-\mathrm{MRS}$ studies reported increased cerebral concentrations of Glu (Brown, Singel, Hepburn, \& Rojas, 2013; Joshi et al., 2013) and decreased concentrations of GABA (Cochran et al., 2015; Gaetz et al., 2014; Harada et al., 2011; Kubas et al., 2012; Rojas, Singel, Steinmetz, Hepburn, \& Brown, 2014) supporting that an imbalanced inhibitory/excitatory system exists in individuals with ASD (Brix et al., 2015). In the current study, neurotransmitter concentrations are determined in 
high-functioning adolescents with ASD and a normal developing control group, excluding individuals who have intellectual deficits. This way, factors possibly related to intellectual impairment instead of ASD, are eliminated. Of the aforementioned previous $1 \mathrm{H}-\mathrm{MRS}$ studies, only one was performed with a study population consisting purely of high-functioning adolescents (Joshi et al., 2013). However, in this particular study only measures of Glu concentrations were obtained. Therefore, the current study is the first to explore whether the concentrations of both Glu and GABA are altered in high-functioning adolescents with ASD compared to a normal developing controls possibly indicative of an imbalance of the inhibitory/excitatory system.

\section{Methods}

\section{Participants}

Thirty-three adolescents were included in this study, of which 15 with ASD and 18 controls. The individuals with ASD were recruited from special secondary education school 'de Berkenschutse' (located in Heeze, the Netherlands), and all the controls attended regular secondary school. All the ASD participants were clinically diagnosed according to Diagnostic and Statistical Manual of Mental Disorders, fourth edition (DSM-IV-TR) and based on individual assessment, using the Autism Diagnostic Observation Schedule (ADOS) (Lord et al., 2000). To assess intelligence, the full-scale IQ (FSIQ) was measured using the Wechsler Intelligence Scale for Children third edition (WISC-III) (Wechsler, 2005). In this particular study, all the adolescents with ASD had an FSIQ over 100, therefore meeting the commonly used criteria of high-functioning autism (clinically diagnosed ASD accompanied with a FSIQ of $>75$ ). Finally, to assess behavioral problems the parents/caregivers of each subject filled out the Child Behavior Checklist (CBCL/6-18) (Achenbach \& Rescorla, 2001) yielding three scores: the total score and scores for internalizing and externalizing problems. Four adolescents with ASD used medication: 3 adolescents used methylphenidate, but discontinued medication at least 20 -h before scanning allowing for complete washout. One adolescent with ASD used pipamperon (antipsychotic) and did not discontinue medication. None of the control adolescents used medication.

Two different methods of $1 \mathrm{H}-\mathrm{MRS}$, point resolved spectroscopy (PRESS) and Mescher-Garwood point-resolved spectroscopy (MEGA-PRESS) (Mescher, Merkle, Kirsch, Garwood, \& Gruetter, 1998), were used in all subjects. Creatine (Cr) and Glu were quantified by the PRESS method and GABA was quantified by the MEGA-PRESS spectral editing method. Since some contribution from co-editing macromolecules is expected using this technique, GABA plus macromolecules will be labeled as GABA+. One of the PRESS and five of the MEGA-PRESS acquisitions were excluded 
due to bad spectral quality after visual inspection. Therefore, the total of included subjects per group (PRESS and a MEGA-PRESS) is slightly different (32 vs 28).

For the PRESS group, which contained a total of 32 participants, the ASD group consisted of 15 adolescents (1 female) between 14-18 years old with a FSIQ ranging from 107 to 124. The PRESS control group consists of 17 control adolescents (1 female) between 12-17 years old with a FSIQ ranging from 105 to 135. Significant differences between the ASD and control group were assessed using independent samples t-tests. No significant differences between the two groups were found in age, intelligence and gender. The groups did show significant differences in all the CBCL scores (total, internalizing and externalizing). An overview of these subject characteristics is shown in Table 1. The MEGA-PRESS group contains 28 subjects, and displays highly similar characteristics with significant differences between the controls and ASD individuals in the three CBCL scores (data not shown).

Table 1 Demographic and descriptive data

\begin{tabular}{|c|c|c|c|c|}
\hline & Control & ASD & \multirow[t]{2}{*}{ t-score } & \multirow[t]{2}{*}{$p$-value } \\
\hline & $M(s d)$ & $M(s d)$ & & \\
\hline Gender (male/female) & $16 / 1$ & $14 / 1$ & 0.089 & .930 \\
\hline Age (years) & $15.3(1.4)$ & $16.2(1.4)$ & -1.991 & .056 \\
\hline Intelligence (FSIQ) & $112(7)$ & $117(5)$ & -1.968 & .058 \\
\hline CBCL total & $10(8)$ & $44(24)$ & -5.232 & .001 \\
\hline CBCL internalizing & $3(3)$ & $13(9)$ & -4.099 & .001 \\
\hline CBCL externalizing & $2(2)$ & $9(6)$ & -4.162 & .001 \\
\hline
\end{tabular}

Note: FSIQ = Full Scale Intelligence Quotient; $\mathrm{CBCL}=$ Child Behavior Checklist; Control = control group; ASD $=$ ASD group

\section{MRI and 1H-MRS acquisition}

Magnetic resonance spectroscopy was performed on a 3.0 Tesla scanner (Philips Achieva, Best, The Netherlands). For anatomic reference and segmentation, T1-weighted three-dimensional (3D) turbo field echo (TFE) images were acquired with the following parameters: repetition time (TR) $8.2 \mathrm{~ms}$, echo time (echo time)

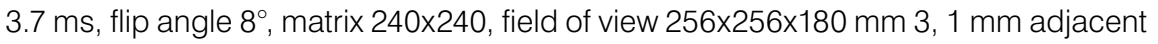
coronal slices. 
$1 \mathrm{H}-\mathrm{MRS}$ spectra were acquired from a single $3 \times 3 \times 3 \mathrm{~cm} 3$ voxel located in the occipital lobe, (Figure 1). The occipital lobe was chosen as it provides the best spectral quality due to its remoteness from the oral region where adolescents typically wear braces. PRESS (TR/ echo time $=2000 / 35 \mathrm{~ms}, 128$ averages, VAPOR water suppression) and MEGA-PRESS (TR/ echo time $=2000 / 68 \mathrm{~ms}, 320$ averages, editing pulses at 1.9 (ON) and 7.46 parts per million (OFF) interleaved in 40 blocks, MOIST water suppression, and 10:40 minutes acquisition time) sequences were used. Additionally, spectra without water suppression were acquired directly after the PRESS (TR/ echo time $=2000 / 35 \mathrm{~ms}, 16$ averages) and MEGA-PRESS (TR/ echo time $=2000 / 68 \mathrm{~ms}$, 8 averages) sequences.

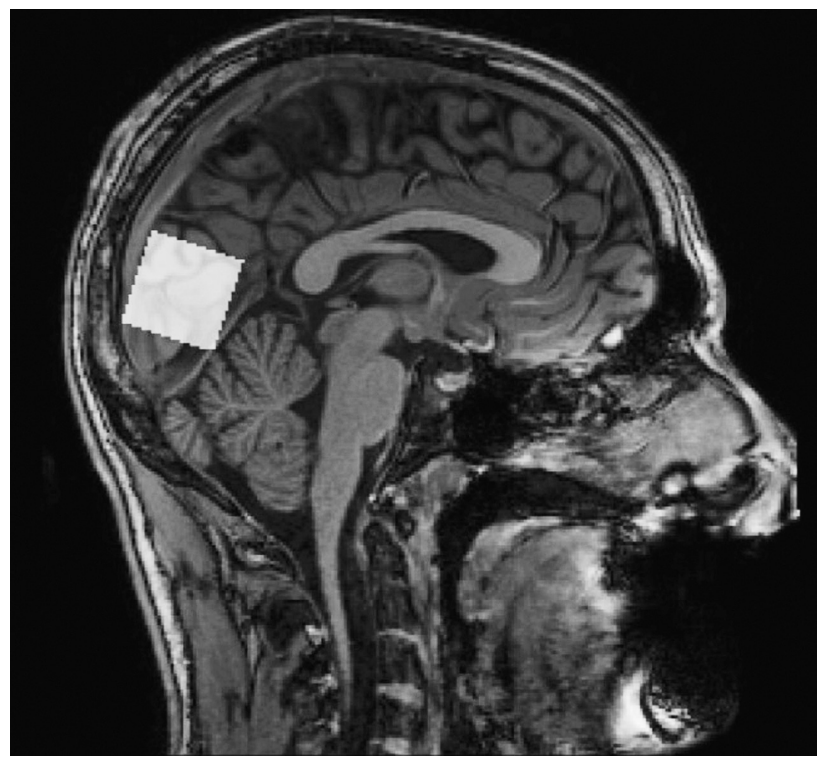

Figure 1 Placement of the proton magnetic resonance spectroscopy (1H-MRS) voxel in the occipital region.

\section{Analysis}

The acquired PRESS data (Figure 2a) of the included subjects was analyzed using LCModel (Version 6.2-2B), which analyzes spectra as a linear combination of basis spectra using a simulated basis set and the acquired MEGA-PRESS spectral editing data (Figure 2b) was analyzed using Gannet (Version 2.0) (Edden, Puts, Harris, Barker, \& Evans, 2014). 
In this study the metabolite concentrations Glu and GABA+ are expressed as a ratio to a reference concentration, $\mathrm{Cr}$. The reference concentration is expected to be stable among the subjects. Both water and $\mathrm{Cr}$ are considered relatively stable (Soares \& Law, 2009), however they can change in severe or chronic disorders (de Graaf et al., 2012). Cr itself is determined relative to water. Furthermore, to examine the imbalance of the inhibitory/excitatory system the ratio GABA+/Glu is determined.
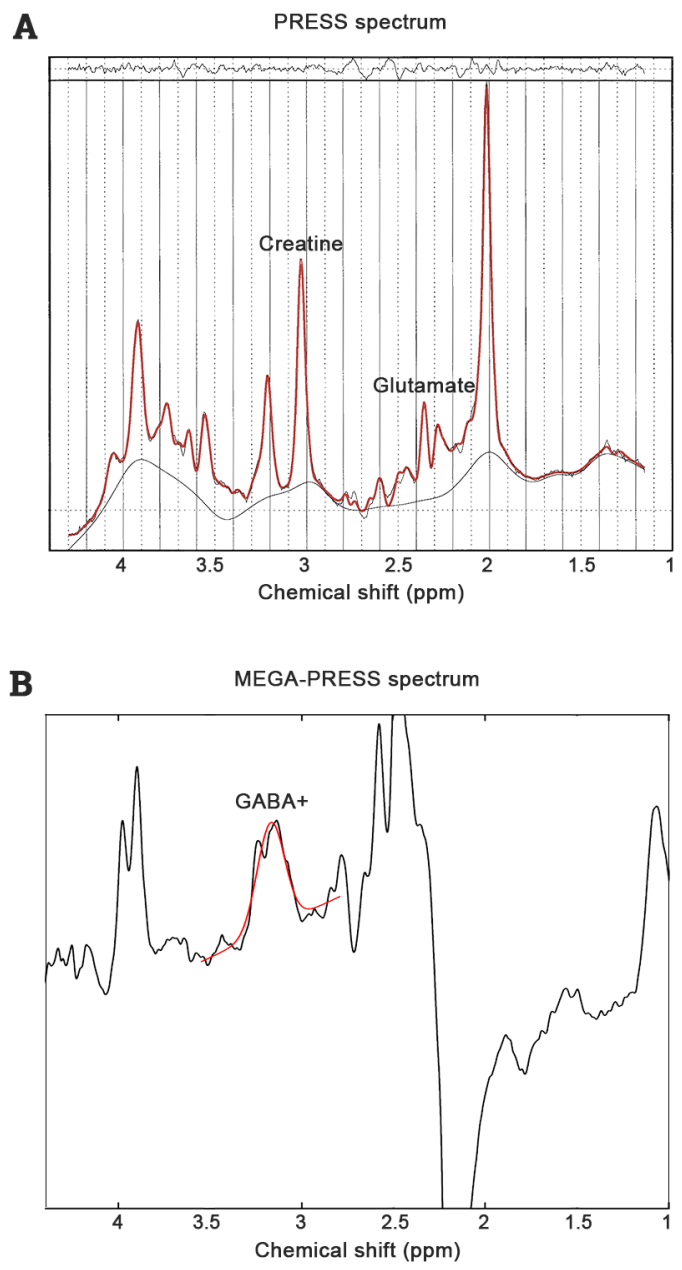

Figure 2 Plots of the (A) Point resolved spectroscopy (PRESS) spectrum and (B) MescherGarwood point resolved spectroscopy (MEGA-PRESS) edited spectrum. In both cases the red line is the fit of the data. 
Concentrations of specific metabolites can vary in different tissues (Bhattacharyya, Phillips, Stone, \& Lowe, 2011). Therefore, the measurements of the metabolite concentrations can be highly influenced by the composition of the $27 \mathrm{ml} 1 \mathrm{H}-\mathrm{MRS}$ voxel. To check whether this is the case in this study, the brain was segmented (Ashburner \& Friston, 2005) into gray matter, white matter and cerebrospinal fluid using Statistical Parametric Mapping v12. To account for potential differences in voxel composition, first the metabolite concentrations are corrected for cerebrospinal fluid. Subsequently, to account for differences in the gray matter and white matter fractions, these fractions are included as covariates in the regression analysis.

Multiple linear regression was performed on the acquired data using the Statistical Package for the Social Sciences (IBM SPSS, v22). As dependent variables the Glu/ $\mathrm{Cr}, \mathrm{GABA}+/ \mathrm{Cr}$ and GABA+/Glu concentrations were taken. The independent variable was the diagnostic group of the participants (controls vs ASD). Moreover, to account for voxel composition, age, intelligence and CBCL scores differences between the groups, gray matter and white matter fractions, age, FSIQ and CBCL total scores, respectively, were added as covariates to the regression model.

\section{Results}

The multiple regression analysis (Table 2$)$ of $\mathrm{Glu} / \mathrm{Cr}(\mathrm{F}(6,25)=3.264, \mathrm{p}=.016, \mathrm{R} 2=.439$ ) revealed a significant higher Glu/Cr for individuals with ASD compared to controls $(p=.026)$. Furthermore, the multiple regression analysis of the GABA+/Glu ratio $(F(6,20)=3.418, p=.017, R 2=.506)$ showed a significant lower concentration for individuals with ASD compared to controls $(p=.006)$. Finally, the multiple regression analysis of $\mathrm{GABA}+/ \mathrm{Cr}$ concentration $(\mathrm{F}(6,21)=1.717, \mathrm{p}=.166, \mathrm{R} 2=.329) \mathrm{did}$ not yield significant differences between the ASD and the control group $(p=.067)$.

An independent samples t-test showed that the cerebrospinal fluid -corrected $\mathrm{Cr}$ concentrations did not significantly differ among the ASD group $(6.03 \pm .43)$ and the controls (5.91 \pm .52$), p=.525$. 
Table 2 Multiple linear regression analysis for $\mathrm{Glu} / \mathrm{Cr}$, GABA+/Glu and $\mathrm{GABA}+/ \mathrm{Cr}$

\begin{tabular}{|l|c|c|c|c|c|c|c|c|c|}
\hline & \multicolumn{3}{|c|}{ Glu/Cr } & \multicolumn{3}{c|}{ GABA+/Glu } & \multicolumn{3}{c|}{ GABA+/Cr } \\
\hline & B & SE B & $\beta$ & B & SE B & $\beta$ & B & SE B & $\beta$ \\
\hline Diagnostic group & .148 & .063 & $.581^{\star}$ & -.019 & .006 & $-.753^{\star \star}$ & -.014 & .007 & -.526 \\
\hline
\end{tabular}

Note: $\mathrm{Glu}=$ glutamate $; \mathrm{GABA}+=$ gamma-aminobutyric acid plus macromolecules $; \mathrm{Cr}=$ creatine $B=$ regression coefficient $;$ SE $B=$ standard error of $B ; \beta=$ relative influence of regression coefficients. ${ }^{*} \mathrm{p}<.05 ;{ }^{* \star} \mathrm{p}<.01$.

\section{Discussion}

The aim of this study was to investigate whether high-functioning adolescents with ASD have altered concentrations of inhibitory and excitatory neurotransmitters compared to controls.

The multiple linear regression analysis revealed two significant differences in metabolite concentrations of the ASD group compared to the normal developing controls; an increase of Glu/Cr concentration and a decrease of GABA+/Glu concentration. As we observed these changes in participants without cognitive decline, it is safe to conclude that the observed alterations are not due to differences in cognitive performance. In this study, no significant differences were found in $\mathrm{Cr}$ between the ASD group and controls, which indicates that the elevation of $\mathrm{Glu} / \mathrm{Cr}$ was not found due to differences in Cr. Furthermore, since the analysis accounts for cerebrospinal fluid, white matter and gray matter fractions, the findings of Glu/Cr and GABA+/Glu are not driven by differences in voxel composition between the two groups.

The Glu/Cr concentration in high-functioning adolescents with ASD is shown to be significantly higher compared to controls. Increased Glu concentration in individuals with ASD has been reported in previous 1H-MRS studies (Brown et al., 2013; Joshi et al., 2013). Also, increased Glu levels in blood plasma has been reported (Aldred et al., 2003; Tu et al., 2012), however it is unclear whether there is an unambiguous relation between these levels and the concentration measured in the brain, as Glu is unable to cross the blood-brain barrier (Tirouvanziam et al., 2012). Glu is the most abundant excitatory neurotransmitter in the brain, inducing activation by binding to post-synaptic receptors. High levels of Glu concentrations can cause excitotoxicity, the process of 
damaging neurons due to over-activity, which has been previously suggested to be associated with at least a subgroup of ASD (M. Evers \& Hollander, 2008).

In this study the concentration of GABA+/Glu is significantly lower in the ASD group, which suggests that GABAergic activity is decreased in the ASD group while glutamatergic activity is increased compared to the controls. Previously, it has been suggested that an imbalance of the inhibitory/excitation system would be located especially in the frontal lobe (Harada et al., 2011). However, in this study it is shown that the effect is observable in the occipital region suggesting that the imbalance of inhibition and excitation is widespread across the brain.

Decreases of GABAergic activity has been reported in several previous $1 \mathrm{H}-\mathrm{MRS}$ ASD studies (Gaetz et al., 2014; Harada et al., 2011; Kubas et al., 2012; Rojas et al., 2014). Also, elevated plasma GABA levels in ASD have been previously reported (Dhossche et al., 2002; El-Ansary \& Al-Ayadhi, 2014). However, as GABA is also unable to cross the blood-brain barrier, a straightforward relationship between the plasma levels and the measured neurotransmitter concentrations is complicated. GABA is the major inhibitory neurotransmitter in the brain, thus preventing the formation of action potentials. A reduction of GABAergic inhibitory activity could lead to hyper-excitability of cortical minicolumnar circuits; vertical columns of functionally related neurons which are important for the flow of excitatory/inhibitory information in the cortex (Mountcastle, 1997). The aforementioned hyper-excitability causes increased 'noise' in cortical systems (Rubenstein \& Merzenich, 2003), which in turn could lead to atypical brain development. A possibly related finding is that the cortical minicolums are shown to be narrower in individuals with ASD (Casanova, Buxhoeveden, \& Gomez, 2003).

The current study has a few limitations. First, the GABA concentration is measured with the contribution of co-editing macromolecules. Therefore, differences in macromolecules across participants can affect the results. However, the macromolecules contribution is presumably constant in all the participants (Bogner et al., 2010). Second, using the $1 \mathrm{H}-\mathrm{MRS}$ technique, tissue levels of neurotransmitter concentrations are obtained rather than the amount or activity of neurotransmitter receptors which are responsible for the inhibitory/excitatory regulation. Last, our voxel was positioned in the occipital lobe, selected for optimal spectral quality, rather than behavioral relevance. However, as we do find significant effects, despite of the occipital placement of the voxel, the observed neurotransmitter effects might be global and of substantial nature. 


\section{Conclusion}

In the present study, changes in the concentrations of $\mathrm{Glu} / \mathrm{Cr}, \mathrm{GABA}+/ \mathrm{Glu}$ and $\mathrm{GABA}+/ \mathrm{Cr}$ in high-functioning adolescents with ASD compared to controls were observed. Increased $\mathrm{Glu} / \mathrm{Cr}$ and decreased GABA+/Glu was found in the occipital region of high-functioning individuals with $A S D$ compared to the normal developing controls, suggesting that changes in the inhibitory and excitation system may contribute to the atypical brain development in ASD. 



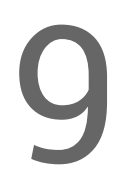

GENERAL DISCUSSION 

Since the publication of Kanners first paper on Autistic children in 1943, much research has focussed on phenotyping, genotyping, describing and investigating diagnostic measures and treatment of individuals with a disorder on the autism spectrum (type: 'autism[Title]' in Pubmed and 18810 result are found). However, most of this research is focussed on children/childhood and far less is known of how ASD manifests itself in high-functioning adolescents. Despite the growing need for and known difficulties in diagnosing these high-functioning adolescents, in clinical practice and scientific research little guidance is offered. This lack of diagnostic recommendations, the uncertainties about the neurocognitive profile and the still unknown aetiology in high-functioning adolescents with ASD, triggered our interest in doing research on this specific subject. We were especially interested in the compensatory mechanisms these adolescents are often able to apply in more structured settings, and therefore, throughout this thesis, we placed an extra focus on the difference in behaviour in the laboratory setting and in real life situations.

The first aim of this thesis was to investigating the use of diagnostic instruments and the neurocognitive profile of high-average intelligent adolescents with ASD.

Comparing the results of the frequently used Achenbach instruments (CBCL, YSR, TRF) ((Achenbach, 1996; Achenbach, 1997a, 1997b) with the ADOS (Autism Diagnostic Observation Schedule) (Falkmer et al., 2013) as gold standard, we found especially the CBCL and the TRF valid as screening instruments. Sensitivity and specificity were satisfactory. The discrepancies between CBCL (parent reports) and TRF (teacher reports) can be of additional diagnostic value. The use of a screening instrument is imperative in clinical practice in which cost restraints necessitate reducing costly, laborious assessments to those who are at risk. Also early assessment of comorbid psychiatric disorders is highly relevant in the treatment of autism (Vannuchi et al., 2014).

The findings in chapter 3 (in which we assessed mentalizing skills and emotion recognition abilities) and chapter 4 (assessing executive function) show comparable results. When high average intelligent adolescents with ASD are assessed in the relative structured environment of the laboratory the results are generally comparable with control subjects. Yet when reports of daily life function are used sharp discrepancies with controls occur. This may be explained by the use of more explicit cognitive or verbally mediating reasoning techniques by high-functioning adolescent with ASD; compensation techniques that may work in more explicit defined tasks, but not on the level of multisensory information processing that is needed in real life social interaction. Thus it is important to realize that when assessing high-average intelligent adolescents with ASD in the laboratory setting of a psychological 
assessment, results can be biased towards the positive side and must always be complemented by reports on or observations in real life.

Finally our theoretical approach in chapter 5 emphasises the importance of impairments in the working memory system as key characteristic of ASD. Working memory systems play a central role in online processing of complex cognitive information and may also play a role in social cognition and interpersonal interaction. We concluded that within the working memory system specific problems of spatial working memory are often seen in adolescents with ASD. These problems increase when information is more complex and greater demands on working memory are made. Again here the concept of cognitive reserve capacity (Moretti et al., 2012; Smith, 2013) and thus compensation is essential. Only with overload of weaker systems and the inability to compensate, a laboratory investigation will show the same results as observed in the daily life practice of e.g. school.

The second aim of this thesis was to gain more insight in the mechanisms underlying this disorder in high-functioning adolescents with a disorder on the autism spectrum.

We examined the working memory brain network integrity in high-functioning adolescents with ASD. Functional MRI was performed in using an n-back task and in resting state. The analysis of the behavioural data revealed deficits in working memory performance in ASD, but only when tested to the limit, confirming also in this study the compensatory mechanisms. Adolescents with ASD showed a loss of global efficiency, suggesting an increase of brain network costs, which may be a neuronal equivalent of the same compensatory process. Since the fMRI efficiency measures correlate with the behavioural data indicative of the severity of the disorder (lower efficiency is related to more social problems), compelling evidence is provided that links connectivity with the behavioural phenotype of ASD.

In a second type of analysis of the resting state data we found changes for adolescents with ASD in the time signals of the brain resting state networks, the so-called neurodynamics. Specifically we found differences in neurodynamics from the ventral stream network to the executive network: a pathway involving cognitive, executiveand emotion-related cortices. This impaired temporal neurodynamics suggests failing bridging of the emotional states regulated in the ventral stream to the decisionmaking oriented executive control system and may therefore be described as a more rigid system in terms of the emotional-executive bridge. An additional finding was that we could only find these changed neurodynamics after a working memory task (n-back task) was given and resting state was repeated. In our study, changes in resting-state $\mathrm{fMRI}$ temporal neurodynamics are therefore triggered by challenging 
the cognitive state prior to resting-state analysis and thus represent an activated state where compensation is more difficult.

Finally MR spectroscopy was performed and directed to two major neurotransmitters: glutamate, the excitatory neurotransmitter and gamma aminobutyric acid (GABA), the main inhibitory neurotransmitter. We found significantly higher glutamate and lower GABA concentrations in the ASD group compared to the controls. These results imply that imbalanced neurotransmitter levels of excitation and inhibition are associated in high-functioning adolescents with ASD. This imbalance leads to hyperexcitability causing increased 'noise' in cortical systems, which also may be related to the constant compensatory tendency in these youngsters.

Several limitations to the studies in this thesis have to be discussed.

- First of all, although power analyses considered 24 participants per group, the number of patients included is limited. The subgroup included in the fMRI study is even smaller. Nonetheless, our studies have gained validity by the homogeneity of the study population: all patients have a high average intelligence, all diagnosis confirmed using the ADOS, no psychiatric comorbidity, only adolescents with a narrow range of age. Therefore the results have representative value for the subpopulation of high-functioning adolescents with ASD. A confirmation of our findings using larger sample size would be interesting.

- The population of high average intelligent adolescents with ASD were only compared to a control population of normal developing adolescents. An interesting topic would be to compare our results also with another clinical sample, such as other subgroups of ASD or patients with Attention Deficit Hyperactivity Disorder. Especially the interpretation of the MRI results would benefit as it would demonstrate the specificity of the obtained network changes.

- When our study was designed, the DSM-IV was the operational version and the diagnosis and inclusion of our patients was based on DSM-IV criteria. It is an open question whether our results would be different using the current DSM-5.

- There is a 6.5 Full-scale IQ-points difference between the controls and the patients with ASD, with higher scores for the adolescents with ASD. Due the homogeneous composition of both groups (and resulting low SD's) the difference is about $1 \mathrm{SD}$. We cannot exclude the possibility that this may have influenced some of the results, although we do not consider this a realistic hazard. 


\section{Clinical implications and further directions}

- Firstly, diagnostic procedures must take into account the compensatory mechanisms that especially occur in structured laboratory investigations in high-functioning adolescents with ASD. Such methods must always be complemented with real life observations and by-proxy reports of significant others.

- The demonstrated compensatory strategies are not always functional. For example, the use of more explicit cognitive or verbally mediating reasoning techniques may work in more explicit defined tasks, but not on the level of multisensory information processing that is needed in real life social interaction. Nonetheless the use of such strategies demonstrate cognitive reserve capacity (Moretti et al., 2012; Smith, 2013) and possibly underlying brain plasticity that may guide new treatment strategies.

- The Achenbach instruments, specifically the CBCL and TRF may provide important information when used as a screening tool in this specific population.

- Our MRI studies provide results that may enhance our understanding of neuronal mechanisms that underlie important characteristics of ASD. Possibly the concept of neurodynamics is essential. Brain structure, BOLD-based activation patterns and the brain network topology are not different from controls. However brain networks are not stable topographic entities but are fluctuating patterns that show understandable time-order relationships. The causal time relations are different in ASD compared to controls and these differences are understandable in terms of the known cortical architecture and are correlated with ASD characteristics. Interestingly the MR spectroscopy shows a 'noisy brain' in terms of the balance between excitatory and inhibitory neurotransmitters. This may somehow be linked to the constant attempt of using compensatory strategies.

- Future studies might concentrate more on combining research methods. This may give researchers and clinicians more insight into the relationships between behaviour, cognition (inside and outside the laboratory), and the functional integrity of the brain. Moreover, studying behaviour, cognition, and the brain in the same cohort will make it easier to interpret $f M R I$ results and the effect of (dys) functional neural networks on everyday living and potential compensation strategies. These insights may help researchers and clinicians to develop and use better intervention techniques for this group of important pervasive developmental disorders. 


\section{Conclusion}

The studies presented in this thesis aimed to investigate the use of diagnostic instruments and the neurocognitive profile of high-average intelligent adolescents with ASD, and gain more insight in the mechanisms underlying this disorder in these teens. High-functioning adolescents with ASD do experience many social cognition and executive functioning difficulties in daily life situations, however, these difficulties cannot always be objectified in a (neuro)psychological assessment. In these more structured settings these intelligent teens are able to apply compensation strategies, although these strategies are often not functional on the level of multisensory information processing that is needed in real life social interaction. In general, this thesis shows that structured (neuro)psychological assessment in high-functioning adolescents with ASD should always be complemented with real life observations and by-proxy reports of significant others, to get a full 'Aha-erlebnis' and as a psychologist be able to give good advice. 

SUMMARY | SAMENVATTING

SOCIETAL RELEVANCE

ABBREVIATIONS

REFERENCES

DANKWOORD

CURRICULUM VITAE

DONDERS GRATUATE SCHOOL

FOR COGNITIVE NEUROSCIENCE SERIES 



\section{Summary | samenvatting}

In Chapter 1 we introduce the topic, i.e. neurocognitive function of high-functioning adolescents with Autism Spectrum Disorder (ASD).

In Chapter 2, the main aim was to investigate the reliability of the Achenbach instruments (CBCL, YSR, TRF) as a screening tool for high-functioning adolescents with ASD. Although there are several specific screening instruments for ASD, they all lack the advantage of containing self-report and parent/teacher - report questionnaires and cannot screen for comorbid psychiatric disorders. In our study, we investigated the ability of the Achenbach instruments to discriminate a group of high-functioning adolescents with a disorder on the autism spectrum from a group of typically developing teens, assessing the correlation between problem behaviour and the ADOS in the autism group, and investigating the discrepancies between parent-, self-, and teacher reports. Although parent reports showed high scores on all CBCL subscales in the ASD group, an elevated pattern on the Withdrawn-depressed-, Social-, Thought-, Attention-, Internalizing- and Total-problems subscales appeared, with respectively moderate and high sensitivity and specificity values. This supports the use of the CBCL as a screening tool for high-functioning ASD adolescents. However, the predictive value of the YSR as a screening tool for ASD adolescents, was poor and makes it unfit for this specific purpose. Informant discrepancies were found between teachers and parents / adolescents with ASD. Although these differences were often ignored in the past, they will give clinicians more insight in the variations of the adolescents' behaviour across settings, and about the differences between the informants' perception and evaluation of this behaviour. This makes it possible to make a good distinction between context-free and context-specific behavioural characteristic of the adolescents, and does acknowledge possible differences in perception of this behaviour between the adolescent and his/her parent/teacher.

In chapter 3, the mentalizing skills and emotion recognition abilities of 21 highfunctioning adolescents with ASD and 21 matched controls were investigated. The results confirm social impairment in daily life situations in adolescents with ASD, but were not found during task related performance. This discrepancy is often found in high-functioning individuals with ASD. In chapter 3, it was hypothesized that this discrepancy may be explained by the use of more explicit cognitive or verbally mediating reasoning techniques by high-functioning adolescent with ASD; compensation techniques that may work in more explicit defined tasks, but not on the level of multisensory information processing that is needed in real life social interaction. Also, adolescents with ASD may have a lesser tendency to search for and 
use social information from multiple simultaneous and dynamic sources in social situations, even though they might possess the ability to comprehend them.

Chapter 4 focused on investigating the relationship between ASD and executive functioning in daily life and laboratory settings. High-functioning adolescents with an Autism Spectrum Disorder (ASD) often show Executive Function deficits, which may contribute to or even underlie their ASD symptomatology. Although our results show that ASD adolescents have more EF deficits than controls in daily life situations, these deficits could not be confirmed by neuropsychological measurements and were unrelated to ASD symptomatology as measured by the Autism Diagnostic Observation Schedule (ADOS). We suggest that although in more structural settings EF performance may be unimpaired, a failure to activate compensation techniques (that are also mentioned in chapter 3 ) in combination with a lesser or missing tendency to approach, explore and experiment in peer and social situations, may lead to a less sophisticated and flexible use of EFs in high-functioning adolescents with ASD in real life situations.

Chapter 5 contains a review article on the neuropsychological and (functional) brain imaging studies on working memory in adolescents with ASD. Working memory is a temporary storage system under attentional control. It is believed to play a central role in online processing of complex cognitive information and may also play a role in social cognition and interpersonal interactions. Adolescents with a disorder on the autism spectrum display problems in precisely these domains. We concluded that within the working memory system specific problems of spatial working memory are often seen in adolescents with ASD. These problems increase when information is more complex and greater demands on working memory are made. Neuroimaging studies indicate a more global working memory processing or connectivity deficiency, rather than a focused deficit in the prefrontal cortex.

In chapter 6, we examined the working memory brain network integrity in highfunctioning adolescents with ASD. Functional MRI was performed using an n-back task and in resting state. The analysis of the behavioural data revealed deficits in working memory performance in ASD, but only when tested to the limit. Adolescents with ASD showed lower binary global efficiency in the working memory network than the control group with n-back and resting state data. This loss of global efficiency suggests an increase of network costs, which may be a sign of the same compensatory process. Since the fMRI efficiency measures correlate with the behavioural data indicative of the severity of the disorder (lower efficiency is related to more social problems), compelling evidence is provided that links connectivity with the behavioural phenotype of ASD. 
In chapter 7, we examined brain resting state networks in high-functioning adolescents with ASD. The aim was to test whether high-functioning adolescents with ASD have an abnormal resting state functional connectivity. We performed spatial and temporal analyses on Resting-State Networks (RSNs) in 13 high-functioning adolescents with ASD and $13 \mathrm{IQ}$ - and age matched controls. We found that controls and high-functioning adolescents with ASD display very similar patterns and strengths of resting-state connectivity. However, in the temporal dynamics of this connectivity, we did find differences in the causal effect properties of RSNs originating in the temporal neurodynamics from the ventral stream network to the executive network: a pathway involving cognitive, executive- and emotion-related cortices. This impaired temporal neurodynamics suggests failing bridging of the emotional states regulated in the ventral stream to the decision-making oriented executive control system and may therefore be described as a more rigid system in terms of the emotional-executive bridge. An additional finding was that we could only find these changed neurodynamics after a working memory task (n-back task) was given and resting state was repeated. In our study, changes in rsfMRI temporal neurodynamics are therefore triggered by challenging the cognitive state prior to resting-state analysis and thus represent an activated state where compensation is more difficult.

Besides differences in brain network integrity and temporal dynamics, previous studies proposed that alterations in excitatory/inhibitory neurotransmitters might play a crucial role in autism spectrum disorder (ASD). In chapter 8, the main aim was to investigate whether high-functioning adolescents with ASD have altered Glu and GABA neurotransmitter concentrations. We found that significantly higher $\mathrm{Glu} / \mathrm{Cr}$ and lower GABA+/Glu concentrations in the ASD group compared to the controls. GABA is the major inhibitory neurotransmitter in the brain, thus preventing the formation of action potentials. A reduction of GABAergic inhibitory activity could lead to hyper-excitability of cortical minicolumnar circuits; vertical columns of functionally related neurons which are important for the flow of excitatory/inhibitory information in the cortex. The hyper-excitability causes increased 'noise' in cortical systems, which in turn could lead to atypical brain development. These results imply that imbalanced neurotransmitter levels of excitation and inhibition are associated in high-functioning adolescents with ASD.

Finally chapter 9 is the general discussion in which we attempt to reflect on the aims of this study. 


\section{Samenvatting}

Sinds de publicatie van Kanners eerste artikel over Autistische kinderen in 1943, heeft veel onderzoek bij mensen met een stoornis binnen het autisme spectrum (ASS) zich gericht op de fenotypering, genotypering, en het omschrijven en onderzoeken van diagnostische en therapeutische interventies (typ: 'autism[Title]' in Pubmed en 18810 resultaten worden gevonden). Hoewel er dus al veel onderzoek is gedaan naar ASS, heeft het meeste onderzoek zich gericht op kinderen/ de kindertijd en is er veel minder bekend over hoe Autisme Spectrumstoornis (ASS) zich presenteert in hoog-functionerende adolescenten. Dit terwijl er juist een groeiende behoefte is naar aanwijzingen vanuit de wetenschap/ klinische praktijk voor evidence based handelen, door de toenemende vraag naar en de complexe diagnostiek die deze leeftijdsgroep met zich meebrengt. Het uitblijven van diagnostische richtlijnen, de onbekende etiologie en de onduidelijkheden rondom het neurocognitieve profiel bij deze groep van hoog-functionerende adolescenten met ASS, wekte onze interesse om juist onderzoek te gaan doen bij deze doelgroep. Onze speciale interesse ging daarbij uit naar de compensatie mechanismen die deze adolescenten vaak kunnen toepassen in een gestructureerde omgeving. In deze thesis is er dan ook veel aandacht voor het verschil in gedrag in meer gestructureerde omgevingen (zoals een onderzoek setting) en het gedrag in het dagelijks leven.

Het doel van dit onderzoek was tweedelig; eerst het onderzoeken van het gebruik van diagnostische instrumenten bij en het neurocognitieve profiel van (boven) gemiddeld intelligente jongeren met ASS, en ten tweede, om meer inzicht te krijgen in de mechanismen achter deze stoornis bij deze hoog-functionerende groep.

In Hoofdstuk 1 introduceren we het onderwerp van onze studies: de neurocognitieve functies van adolescenten met normale intelligentie en een stoornis in het autisme spectrum.

In Hoofdstuk 2, rapporteren we de resultaten van het onderzoek naar de betrouwbaarheid en validiteit van de door Achenbach ontworpen vragenlijsten (CBCL, YSR, TRF) als screening instrument voor adolescenten met normale intelligentie en een stoornis in het autisme spectrum. Hoewel er al verschillende screeningsinstrumenten bestaan die gebruikt worden bij autisme, ontbreken de mogelijkheden van zelfrapportage, en 'by proxy' rapportage door zowel de ouders als de leerkrachten. Bovendien screenen bestaande instrumenten niet voor psychiatrische comorbiditeit. In onze studie onderzochten we het vermogen van de Achenbach instrumenten om de groep hoog-functionerende adolescenten met ASD te differentiëren van normaal functionerende adolescenten. De resultaten van de Achenbach instrumenten werden 
daartoe gecorreleerd met de ADOS die als 'gold standard' werd gebruikt. Daarnaast werden ook verschillen onderzocht tussen de zelfrapportage (YSR), de rapportages van de ouders (CBCL) en die van de leerkrachten (TRF). Ouders rapporteerden verhoogde scores met name op de subschalen: Teruggetrokken/Depressief, Sociaal, Denkstoornissen, Aandachtsproblemen, Internaliserend gedrag en de Totale Problemen Schaal. De sensitiviteit van de CBCL bleek acceptabel en de specificiteit hoog. Deze resultaten ondersteunen het gebruik van de ouderschaal $(C B C L)$ als screeningsinstrument voor hoog-functionerende adolescenten met ASD. Het onderscheidend vermogen van de YSR (zelfrapportage) bleek te gering voor dit specifieke doel. Er waren verder opvallende verschillen gevonden tussen de ouderrapportage (CBCL) en de rapportages van leerkrachten (TRF). Zulke discrepanties zijn veelal genegeerd, maar ons inziens vormen deze een belangrijke aanvullende bron van informatie; ze kunnen clinici inzicht geven in de variaties in gedrag per setting en context. Zo toont het verschillen tussen de zelfperceptie en de omgevingsevaluatie van gedrag.

In hoofdstuk 3, werden de vermogens tot mentaliseren en emotie-herkenning onderzocht bij 21 normaal intelligente adolescenten met autisme spectrum en vergeleken die met 21 gematchte controles. De resultaten bevestigen sociale stoornissen van de hoog-functionerende adolescenten met ASD in het dagelijks leven terwijl deze tijdens aangeboden taken bij een formeel psychologisch onderzoek niet teruggevonden worden. Deze discrepantie is typerend voor hoog-functionerende adolescenten met ASD en kan verklaard worden door het gebruik van compensatoire cognitieve, verbale redeneer-vaardigheden die functioneel zijn in een gestructureerde omgeving maar die niet werken in de multisensorische, multitasking omgeving van de real-life sociale interacties. Het is ook mogelijk dat hoog-functionerende adolescenten met ASD restrictief zijn in het verzamelen van sociale informatie, alhoewel ze wel het vermogen hebben de informatie te begrijpen.

Hoofdstuk 4 richt zich op het onderzoeken van relaties tussen hoog-functionerende adolescenten met ASD en executieve functies zowel in het dagelijks leven als in formele laboratorium situaties. Stoornissen in de executieve functies treden typisch op bij autisme spectrum stoornissen. Onze resultaten toonden meer stoornissen in de executieve functies in het dagelijks leven aan, die echter niet bevestigd werden tijdens formeel neuropsychologisch onderzoek. Evenals in het vorige hoofdstuk is onze hypothese dat de executieve functies ongestoord zijn in voorspelbare en gestructureerde settingen waar compensatoire redeneerfuncties ingezet kunnen worden. In dagelijkse sociale situaties is dat moeilijker en de combinatie van falende compensatie en een verminderde neiging tot exploratie van sociale 'cues' leidt dan tot minder flexibel gebruik van executieve functies. 
Hoofdstuk 5 is een review artikel waarin een overzicht en beoordeling wordt gegeven van de literatuur over de neuropsychologische en functionele MRI studies in de groep adolescenten met normale intelligentie en een stoornis in het autisme spectrum. Specifiek richt het review zich op stoornissen in het werkgeheugen. Het werkgeheugen is een tijdelijk opslagsysteem dat sterk gerelateerd is aan aandachtsfuncties. Het is een centrale schakel in de continue centrale verwerking van complexe cognitieve informatie, en is belangrijk bij de verwerking van complexe sociale cognitieve informatie en speelt mogelijk een belangrijke rol in sociale cognitie en sociale interacties. Dit zijn juist de domeinen waar adolescenten met een autisme spectrum stoornissen de meeste problemen ondervinden. Deze problemen nemen toe wanneer informatie meer complex is en als er meer eisen gesteld worden aan het werkgeheugen. De neuroimaging studies wijzen eerder op globale afwijkingen in het breinnetwerk, meer dan focale afwijkingen in de prefrontale cortex.

In hoofdstuk 6, werd de netwerk integriteit onderzocht in relatie tot het werkgeheugen van adolescenten met normale intelligentie en een stoornis in het autisme spectrum. Functionele MRI werd geanalyseerd zowel na activatie met een werkgeheugentaak (n-back) als in 'resting state' (zonder specifieke cognitieve activatie). Analyse van de neuropsychologische data toonde weliswaar stoornissen in het werkgeheugen van de adolescenten met autisme, maar alleen na 'testing to the limit', dus na falende compensatie. Zowel na activatie met de n-back taak als in 'resting state' zien we verminderde 'global efficiency' bij de adolescenten met autisme vergeleken met controles. Verlies van 'global efficiency' suggereert een toename van netwerk belasting; een fenomeen dat mogelijk te herleiden is tot het constant inzetten van compensatoire mechanismen. De veranderingen in 'global efficiency' correleerden overigens met de neuropsychologische resultaten en met de ernst van sociale problemen. Verandering van netwerk efficiency is dus een van de neuronale correlaten voor autisme bij adolescenten met normale intelligentie.

In hoofdstuk 7, wordt een technologisch meer geavanceerde methode toegepast op de resting state $f M R I$ data van de adolescenten met autisme $(n=13)$ en de controles $(n=13)$. De netwerk architectuur verschilde niet tussen de groepen; Dezelfde type topografie van de netwerken werd gevonden. Dergelijke netwerken zijn echter niet stabiel over tijd, maar tonen fluctuaties, waarbij sommige patronen met elkaar samenhangen (Granger causale benadering). Deze weergave wordt ook aangeduid als 'neurodynamics'. We vonden significante veranderingen in een pathway dat essentieel is bij autisme, namelijk een verminderd neurodynamisch patroon vanuit een netwerk dat aangeduid wordt als 'ventral stream' (een hoofdzakelijk temporaal georiënteerd netwerk dat sterk actief is bij emotionele mechanismen) naar het executieve netwerk dat op het maken van beslissingen en executieve controle 
gericht is. Een dergelijke verandering in netwerk interactie kan wijzen op een meer rigide systeem tussen emotioneel- en executief functioneren, en is belangrijk bij het begrijpen van de kernproblemen bij autisme. Een aanvullende bevinding was dat de netwerk afwijking pas werd gevonden na het 'to the limit' testen van de jongeren; na de n-back taak. Mogelijk worden de netwerk veranderingen pas gezien als compensatie niet meer mogelijk is.

Een laatste benadering is die waarin de MRI gebruikt wordt om zicht te krijgen op metabole processen: MR spectroscopie. In eerdere studies is gesuggereerd dat veranderingen op het niveau van neurotransmitters ten grondslag zouden kunnen liggen aan autisme spectrum stoornissen. In hoofdstuk 8 beschrijven we de verschillen in concentraties van twee essentiële neurotransmitters: de excitatoire neurotransmitter glutamaat en de inhiberende neurotransmitter GABA. We vonden dat de relatieve concentratie van glutamaat $(\mathrm{Glu} / \mathrm{Cr}$ ) duidelijk verhoogd was en de relatieve concentratie van GABA (GABA+/Glu) duidelijk verlaagd was bij de adolescenten met autisme. Deze veranderde balans kan een meer excitatoire organisatie van neuronale systemen induceren in de vorm van hyperexcitabilteit ('a noisy brain'). Deze bevinding biedt naast het verlies van global efficiency en een verminderde neurodynamische interactie tussen ventral stream en executive netwerk een derde inkijk in de hoogstwaarschijnlijk multidimensionele neuronale correlaten van adolescenten met een autisme spectrum stoornis.

Tenslotte biedt hoofdstuk 9 de discussie waarin we met name terugkomen op de twee hoofddoelen van onze studies. Er wordt geconcludeerd dat in het dagelijks leven hoog-functionerende adolescenten met ASS inderdaad veel problemen ervaren op het gebied van sociale cognitie en het executief functioneren. Deze problemen kunnen echter vaak niet geobjectiveerd worden in een (neuro)psychologisch onderzoek. In tegenstelling tot het dagelijks leven, waarbij sociale interactie een beroep doet op multisensorische informatie verwerking en de compensatie strategieën van deze intelligente jongeren onvoldoende lijken te werken; lukt het ze namelijk wél om deze strategieën toe te passen binnen het gestructureerde kader van een (neuro) psychologisch onderzoek. Wat onderzoek in deze thesis dan ook laat zien, is dat gestructureerd (neuro)psychologisch onderzoek bij hoog-functionerende adolescenten met ASS, altijd aangevuld dient te worden met observaties van het gedrag in het dagelijks leven en informatie van ouders/verzorgers/ leerkrachten, om een volledige 'Aha-erlebnis' te verkrijgen en de psycholoog een goed advies kan geven. 



\section{Societal relevance}

\section{Relevance}

In the Netherlands a growing number of high average intelligent adolescents with ASD is in the need for special secondary education (waiting lists are growing). These adolescents often experience many problems in their everyday life, and executive functioning problems are commonly recognized by parents, caregivers and teachers. Diagnostic interviews, observations, and neuropsychological tests, however, are challenging to apply in these high functioning adolescents with ASD. Because of their ability to masquerade their symptoms, especially in clinical or laboratory settings, it is very difficult to diagnose and inventory their problems (Rump, Giovannelli, Minshew, \& Strauss, 2009; Salter, Seigal, Claxton, Lawrence, \& Skuse, 2008). Making a good diagnosis is extra important at this age while, although the social difficulties are independent of age and developmental level, the related psychosocial stress may disproportionally increase during adolescence (Nicpon, Doobay, \& Assouline, 2010; E. Pellicano, 2010b). This is especially noticeable in high-functioning adolescents with ASD, as they more than their low-functioning counterparts, seek and initiate social interaction with peers (Bauminger, Shulman, \& Agam, 2003; Hauck, Fein, Waterhouse, \& Feinstein, 1995). Despite the need for and known difficulties in diagnosing these high-functioning adolescents, in clinical practice and scientific research little recommendations are offered. This is why in the first part of this thesis, we take a specific interest in investigating the use of diagnostic instruments and the neurocognitive profile of high-average intelligent adolescents with ASD.

In the second part of this thesis, we were interested in gaining more insight in the mechanisms behind this disorder in high-functioning adolescents, as to our opinion this is an essential issue for understanding autism and for designing therapies and treatments. Nowadays it is generally recognized that besides the well-known triad of problems, many people with ASD experience problems in executive functioning (Hill, 2004). Given the central role of executive functions in both higher and lower cognitive processes, problems in these functions could provide a good explanation for the symptoms seen in ASD. As working memory is generally seen as a central process in many, if not all executive functions, we propose that it is highly plausible that working memory plays a more leading role in both the social and non-social symptoms seen in ASD. To date, however, studies have failed to give conclusive evidence about the relationship between working memory functioning and the symptoms seen in high-functioning adolescents with ASD. Therefore, in this thesis, we started to explore the role of working memory functioning in high-functioning adolescents' ASD symptomatology. 


\section{Main findings}

In the first part of this thesis, clinical studies are presented that investigated the use of diagnostic instruments and the neurocognitive profile of high-average intelligent adolescents with ASD. We found that high-functioning adolescents with ASD do experience many social cognition and executive functioning difficulties in daily life situations, and that these difficulties cannot always be objectified in a (neuro) psychological assessment. In more structured settings, these intelligent teens are able to apply compensation strategies, although these strategies are often not functional on the level of multisensory information processing that is needed in real life social interaction. In general, this thesis shows that structured (neuro)psychological assessment in high-functioning adolescents with ASD should always be complemented with real life observations and by-proxy reports of significant others, to get a full 'Aha-erlebnis' and as a psychologist be able to give good advice.

The second part of this thesis described research into the mechanisms underlying ASD in high-functioning adolescents. We examined the working memory brain network integrity in high-functioning adolescents with ASD, investigated the neurodynamics of the brain resting state networks, and MR spectroscopy was performed and directed to two major neurotransmitters: glutamate, the excitatory neurotransmitter and gamma aminobutyric acid (GABA), the main inhibitory neurotransmitter. These studies showed evidence for a loss of global efficiency, changes in resting-state $\mathrm{fMRI}$ temporal neurodynamics, and imbalanced neurotransmitter levels of excitation and inhibition associated with ASD in high functioning adolescents, which may be related to the constant compensatory tendency in these youngsters.

\section{Target group}

First of all, the findings of this study are relevant for high-functioning adolescents with $A S D$, as these findings might, in the future, improve diagnostic processes and therapeutic treatments in ASD. This thesis is also of interests for their psychologists; who might benefit from the recommendations and may adjust their standard ASD assessment and therapeutic treatment in these high-functioning teens. Furthermore, our MRI studies provide results that may enhance our understanding of neuronal mechanisms that underlie important characteristics of ASD, and might give new directions for future research on the functional integrity of the brain and the mechanisms underlying ASD. Individuals with ASD may benefit from this ass well, because (in the future) it may give insights for other (more suitable) therapeutic interventions or treatments in this high-functioning group. 


\section{Innovation and future directions}

Since the publication of Kanners first paper on Autistic children in 1943, much research has focussed on phenotyping, genotyping, describing and investigating diagnostic measures and treatment of individuals with a disorder on the autism. However, most of this research is focussed on children/childhood and far less is known of how ASD manifests itself in high-functioning adolescents. The studies presented in this thesis should be considered as explorations on the use of diagnostic instruments and the neurocognitive profile of high-average intelligent adolescents with ASD, and the mechanisms behind this disorder in these teens. We recommend that future studies concentrate more on combining research methods. This may give researchers and clinicians more insight into the relationships between behaviour, cognition (inside and outside the laboratory), and the functional integrity of the brain. Moreover, studying behaviour, cognition, and the brain in the same cohort will make it easier to interpret fMRI results and the effect of (dys)functional neural networks on everyday living and potential compensation strategies. These insights may help researchers and clinicians to develop and use better intervention techniques for this group of important pervasive developmental disorders. 



\section{Abbreviations}

\begin{tabular}{|c|c|}
\hline 1H-MRS & proton magnetic resonance spectroscopy \\
\hline ADOS & autism diagnostic observation schedule \\
\hline ANOVA & analysis of variance \\
\hline ASD & autism spectrum disorder \\
\hline AUC & area under the ROC curve \\
\hline BRIEF & behavior rating inventory of executive function \\
\hline CBCL & child behavior check list \\
\hline $\mathrm{Cr}$ & creatine \\
\hline DLPFC & dorsolateral prefrontal cortex \\
\hline DSM-5 & diagnostic and statistical manual of mental disorders, fifth edition \\
\hline DSM-III & diagnostic and statistical manual of mental disorders, third edition \\
\hline DSM-IV & diagnostic and statistical manual of mental disorders, fourth edition \\
\hline EF & executive function \\
\hline ERT & emotion recognition task \\
\hline FDI & freedom from distractibility index \\
\hline fMRI & functional magnetic resonance imaging \\
\hline FSIQ & full scale intelligence quotient \\
\hline FSL & FMRIB software library \\
\hline GABA & gamma-aminobutyric acid \\
\hline $\mathrm{GABA}+$ & gamma-aminobutyric acid plus macromolecules \\
\hline GLM & general linear model \\
\hline Glu & glutamate \\
\hline HFA & high-functioning autism \\
\hline IC & independent component \\
\hline ICA & independent component analysis \\
\hline LFA & low-functioning autism \\
\hline LR & likelihood ratio \\
\hline MANCOVA & multivariate analysis of covariance \\
\hline MANOVA & multivariate analysis of variance \\
\hline MEGA-PRESS & Mescher-Garwood point resolved spectroscopy \\
\hline MRI & magnetic resonance imaging \\
\hline $\mathrm{PCl}$ & perceptual organisation index \\
\hline PDD-NOS & pervasive developmental disorder-not otherwise specified \\
\hline PIQ & performance intelligence quotient \\
\hline POP & preparing-to-overcome prepotency \\
\hline PRESS & point resolved spectroscopy \\
\hline ROC & receiver operating characteristics \\
\hline RSN & resting state network \\
\hline ToM & theory of mind \\
\hline TRF & teacher report form \\
\hline $\mathrm{VCl}$ & verbal comprehension index \\
\hline VIQ & verbal intelligence quotient \\
\hline WISC-III & wechsler intelligence scale for children \\
\hline WM & working memory \\
\hline YSR & youth self-report \\
\hline
\end{tabular}





\section{References}

Abrahams, B., \& Geschwind, D. (2008). Advances in autism genetics: on the threshold of a new neurobiology. Nat Rev Genet, 9(5), 341-355.

Achard, S., \& Bullmore, E. (2007). Efficiency and cost of economical brain functional networks. Plos Computational Biology, 3(2), 174-183. doi: DOI 10.1371/journal.pcbi.0030017

Achenbach, T. M. (1996). Handleiding voor de Child Behavior Check List (CBCL) 4-18. Version 1/93 2001. (F. C. Verhulst \& J. v. d. Ende, Trans.). Rotterdam: Erasmus UMC- Sophia Kinderziekenhuis.

Achenbach, T. M. (1997a). Handleiding voor de Teather's Report Form (TRF). Version 9/2 2001. (F. C. Verhulst \& J. v. d. Ende, Trans.). Rotterdam: Erasmus UMC- Sophia Kinderziekenhuis.

Achenbach, T. M. (1997b). Handleiding voor de Youth Self-Report (YSR). Version 8/4 2001. (F. C. Verhulst \& J. v. d. Ende, Trans.). Rotterdam: Erasmus UMC- Sophia Kinderziekenhuis.

Achenbach, T. M. (2011). Commentary: Definitely more than measurement error: but how should we understand and deal with informant discrepancies? J Clin Child Adolesc Psychol, 40(1), 80-86. doi: 10.1080/15374416.2011.533416

Achenbach, T. M., \& Rescorla, L. A. (2001). Manual for the ASEBA school-age forms \& profiles. Burlington: University of Vermont, Research Centre for Children, Youth, \& Families.

Akaike, H. (1974). A new look at statistical model identification. IEEE Trans Autom Control 19(6), 716-723.

Aldred, S., Moore, K. M., Fitzgerald, M., \& Waring, R. H. (2003). Plasma amino acid levels in children with autism and their families. J Autism Dev Disord, 33(1), 93-97.

Amaral, D. G., Schumann, C. M., \& Nordahl, C. W. (2008). Neuroanatomy of autism. Trends Neurosci, 31(3), 137-145. doi: S0166-2236(08)00038-6 [pii] 10.1016/j.tins.2007.12.005

Anderson, J. S., Nielsen, J. A., Froehlich, A. L., DuBray, M. B., Druzgal, T. J., Cariello, A. N., . . Lainhart, J. E. (2011). Functional connectivity magnetic resonance imaging classification of autism. Brain, 134(12), 3742-3754.

Apperly, I. A., Carroll, D. J., Samson, D., Humphreys, G. W., Qureshi, A., \& Moffitt, G. (2010). Why are there limits on theory of mind use? Evidence from adults' ability to follow instructions from an ignorant speaker. Quarterly Journal of Experimental Psychology, 63(6), 1201-1217. doi: Pii 915921230 Doi 10.1080/17470210903281582

Ashburner, J., \& Friston, K. J. (2005). Unified segmentation. [Research Support, Non-U.S. Gov't]. Neuroimage, 26(3), 839-851. doi: 10.1016/j.neuroimage.2005.02.018

Asperger, H. (1943). Die "Autistischen Psychopathen" im Kindesalter. Habilitationsschrift Medizinischen Fakultät der Wiener Universität.

Awate, S. P., Win, L., Yushkevich, P., Schultz, R. T., \& Gee, J. C. (2008). 3D cerebral cortical morphometry in autism: increased folding in children and adolescents in frontal, parietal, and temporal lobes. Med Image Comput Comput Assist Interv, 11(Pt 1), 559-567.

Aylward, E. H., Minshew, N. J., Field, K., Sparks, B. F., \& Singh, N. (2002). Effects of age on brain volume and head circumference in autism. Neurology, 59(2), 175-183.

Baddeley, A. (2012). Working memory: theories, models, and controversies. Annu Rev Psychol, 63, 1-29. doi: 10.1146/annurev-psych-120710-100422

Baddeley, A., Della Sala, S., Papagno, C., \& Spinnler, H. (1997). Dual-task performance in dysexecutive and nondysexecutive patients with a frontal lesion. Neuropsychology, 11(2), 187-194.

Banko, E. M., Gal, V., \& Vidnyanszky, Z. (2009). Flawless visual short-term memory for facial emotional expressions. [Research Support, Non-U.S. Gov't]. J Vis, 9(1), 12 11-13. doi: 10.1167/9.1.12

Barbey, A. K., Colom, R., Solomon, J., Krueger, F., Forbes, C., \& Grafman, J. (2012). An integrative architecture for general intelligence and executive function revealed by lesion mapping. Brain, 135(Pt 4), 1154-1164. doi: 10.1093/brain/aws021

Barendse, E. M., Hendriks, M. P., Jansen, J. F., Backes, W. H., Hofman, P. A., Thoonen, G., . . Aldenkamp, A. P. (2013). Working memory deficits in high-functioning adolescents with autism spectrum disorders: neuropsychological and neuroimaging correlates. J Neurodev Disord, 5(1), 14. doi: 10.1186/1866-1955-5-14

Barnea-Goraly, N., Kwon, H., Menon, V., Eliez, S., Lotspeich, L., \& Reiss, A. L. (2004). White matter structure in autism: preliminary evidence from diffusion tensor imaging. Biol Psychiatry, 55(3), 323-326. 
Barnett, L., \& Seth, A. K. (2014). The MVGC multivariate Granger causality toolbox: a new approach to Granger-causal inference. [Research Support, Non-U.S. Gov't]. J Neurosci Methods, 223, 50-68. doi: 10.1016/j.jneumeth.2013.10.018

Barnhill, G. P., Hagiwara, T., Smith, M. B., Simpson, R. L., Brick, M. L., \& Griswold, D. E. (2000). Parent, Teacher, and Self-Report of Problem and Adaptive Behaviors in Children and Adolescents with Asperger Syndrome. Diagnostique, 25(2), 147-167.

Baron-Cohen, S., Hoekstra, R. A., Knickmeyer, R., \& Wheelwright, S. (2006). The Autism-Spectrum Quotient (AQ)--adolescent version. J Autism Dev Disord, 36(3), 343-350. doi: 10.1007/s10803-006-0073-6

Baron-Cohen, S., Leslie, A., \& Frith, U. (1985). Does the autistic child have a "theory of mind"? Cognition, 21(1), 37-46. doi: 0010-0277(85)90022-8 [pii]

Baron-Cohen, S., Wheelwright, S., Hill, J., Raste, Y., \& Plumb, I. (2001). The "Reading the Mind in the Eyes" Test revised version: a study with normal adults, and adults with Asperger syndrome or high-functioning autism. [Research Support, Non-U.S. Gov't Validation Studies]. J Child Psychol Psychiatry, 42(2), 241-251.

Baruth, J. M., Wall, C. A., Patterson, M. C., \& Port, J. D. (2013). Proton magnetic resonance spectroscopy as a probe into the pathophysiology of autism spectrum disorders (ASD): a review. [Review]. Autism Res, 6(2), 119-133. doi: 10.1002/aur.1273

Bauminger, N. (2002). The facilitation of social-emotional understanding and social interaction in high-functioning children with autism: intervention outcomes. [Research Support, Non-U.S. Gov't]. J Autism Dev Disord, 32(4), 283-298.

Bauminger, N. (2004). The expression and understanding of jealousy in children with autism. Dev Psychopathol, 16(1), 157-177.

Bauminger, N., Shulman, C., \& Agam, G. (2003). Peer interaction and loneliness in high-functioning children with autism. [Research Support, Non-U.S. Gov't]. J Autism Dev Disord, 33(5), 489-507.

Bauminger, N., Solomon, M., Aviezer, A., Heung, K., Brown, J., \& Rogers, S. J. (2008). Friendship in high-functioning children with autism spectrum disorder: mixed and non-mixed dyads. [Research Support, Non-U.S. Gov't]. J Autism Dev Disord, 38(7), 1211-1229. doi: 10.1007/s10803-007-0501-2

Bauminger, N., Solomon, M., Aviezer, A., Heung, K., Gazit, L., Brown, J., \& Rogers, S. J. (2008). Children with autism and their friends: a multidimensional study of friendship in high-functioning autism spectrum disorder. [Research Support, Non-U.S. Gov't]. J Abnorm Child Psychol, 36(2), 135-150. doi: 10.1007/ s10802-007-9156-x

Beckmann, C. F., DeLuca, M., Devlin, J. T., \& Smith, S. M. (2005). Investigations into resting-state connectivity using independent component analysis. [Comparative Study Research Support, Non-U.S. Gov't]. Philos Trans R Soc Lond B Bio/ Sci, 360(1457), 1001-1013. doi: 10.1098/rstb.2005.1634

Begeer, S., Gevers, C., Clifford, P., Verhoeve, M., Kat, K., Hoddenbach, E., \& Boer, F. (2011). Theory of Mind training in children with autism: a randomized controlled trial. [Randomized Controlled Trial]. J Autism Dev Disord, 41(8), 997-1006. doi: 10.1007/s10803-010-1121-9

Belmonte, M. K., Allen, G., Beckel-Mitchener, A., Boulanger, L. M., Carper, R. A., \& Webb, S. J. (2004). Autism and abnormal development of brain connectivity. [Research Support, Non-U.S. Gov't Review]. J Neurosci, 24(42), 9228-9231. doi: 10.1523/JNEUROSCI.3340-04.2004

Ben Shalom, D. (2003). Memory in autism: review and synthesis. Cortex, 39(4-5), 1129-1138.

Bergmann, H. C., Rijpkema, M., Fernández, G., \& Kessels, R. P. (2012). Distinct neural correlates of associative working memory and long-term memory encoding in the medial temporal lobe. Neuroimage.

Berthoz, S., \& Hill, E. L. (2005). The validity of using self-reports to assess emotion regulation abilities in adults with autism spectrum disorder. Eur Psychiatry, 20(3), 291-298. doi: 10.1016/j.eurpsy.2004.06.013

Bhattacharyya, P. K., Phillips, M. D., Stone, L. A., \& Lowe, M. J. (2011). In vivo magnetic resonance spectroscopy measurement of gray-matter and white-matter gamma-aminobutyric acid concentration in sensorimotor cortex using a motion-controlled MEGA point-resolved spectroscopy sequence. [Research Support, N.I.H., Extramural Research Support, Non-U.S. Gov't]. Magn Reson Imaging, 29(3), 374-379. doi: 10.1016/j.mri.2010.10.009 
Biederman, J., Petty, C. R., Fried, R., Wozniak, J., Micco, J. A., Henin, A., . . Faraone, S. V. (2010). Child behavior checklist clinical scales discriminate referred youth with autism spectrum disorder: a preliminary study. J Dev Behav Pediatr, 31(6), 485-490.

Blakemore, S. J. (2008). The social brain in adolescence. Nat Rev Neurosci, 9(4), 267-277. doi: 10.1038/ $\mathrm{nrn} 2353$

Blakemore, S. J., \& Choudhury, S. (2006). Development of the adolescent brain: implications for executive function and social cognition. J Child Psychol Psychiatry, 47(3-4), 296-312. doi: 10.1111/j.1469-7610. 2006.01611.x

Bogner, W., Gruber, S., Doelken, M., Stadlbauer, A., Ganslandt, O., Boettcher, U., . . Hammen, T. (2010). In vivo quantification of intracerebral GABA by single-voxel (1)H-MRS-How reproducible are the results? [Research Support, Non-U.S. Gov't]. Eur J Radiol, 73(3), 526-531. doi: 10.1016/j.ejrad.2009.01.014

Boucher, J., \& Mayes, A. (2011). Memory in ASD. In D. Fein (Ed.), The neuropsychology of autism (pp. 139-160). New York: Oxford University Press.

Bramham, J., Ambery, F., Young, S., Morris, R., Russell, A., Xenitidis, K., . . Murphy, D. (2009). Executive functioning differences between adults with attention deficit hyperactivity disorder and autistic spectrum disorder in initiation, planning and strategy formation. [Comparative Study]. Autism, 13(3), 245-264.

Bressler, S. L., \& Seth, A. K. (2011). Wiener-Granger causality: a well established methodology. [Research Support, Non-U.S. Gov't]. Neuroimage, 58(2), 323-329. doi: 10.1016/j.neuroimage.2010.02.059

Brix, M. K., Ersland, L., Hugdahl, K., Gruner, R., Posserud, M. B., Hammar, A., . . Beyer, M. K. (2015). "Brain MR spectroscopy in autism spectrum disorder-the GABA excitatory/inhibitory imbalance theory revisited". Front Hum Neurosci, 9, 365. doi: 10.3389/fnhum.2015.00365

Brosnan, M., Johnson, H., Grawmeyer, B., Chapman, E., \& Benton, L. (2015). Emotion recognition in animated compared to human stimuli in adolescents with autism spectrum disorder. [Research Support, Non-U.S. Gov't]. J Autism Dev Disord, 45(6), 1785-1796. doi: 10.1007/s10803-014-2338-9

Brown, M. S., Singel, D., Hepburn, S., \& Rojas, D. C. (2013). Increased glutamate concentration in the auditory cortex of persons with autism and first-degree relatives: a (1)H-MRS study. [Research Support, N.I.H., Extramural]. Autism Res, 6(1), 1-10. doi: 10.1002/aur.1260

Brüne, M. (2003). Theory of mind and the role of $I Q$ in chronic disorganized schizophrenia. Schizophr Res, $60(1), 57-64$.

Brune, M., \& Brune-Cohrs, U. (2006). Theory of mind--evolution, ontogeny, brain mechanisms and psychopathology. [Review]. Neurosci Biobehav Rev, 30(4), 437-455. doi: 10.1016/j.neubiorev.2005.08.001

Bull, R., \& Andrews Espy, K. (2006). Working memory, executive functioning, and children's methematics. In S. J. Pickering (Ed.), Working memory and education: Academic Press Elsevier Inc.

Bull, R., Phillips, L. H., \& Conway, C. A. (2008). The role of control functions in mentalizing: dual-task studies of theory of mind and executive function. Cognition, 107(2), 663-672.

Burnett, S., Bird, G., Moll, J., Frith, C., \& Blakemore, S. J. (2009). Development during adolescence of the neural processing of social emotion. [Research Support, Non-U.S. Gov't]. J Cogn Neurosci, 21(9), 1736-1750. doi: 10.1162/jocn.2009.21121

Cain, K. (2006). Children's reading comprehension: the role of working memory in normal and impaired development. In S. J. Pickering (Ed.), Working memory and education: Acedemic Press Elsevier Inc.

Campbell, J. M. (1998). Internal and external validity of seven Wechsler Intelligence Scale for Children-Third Edition short forms in a sample of psychiatric inpatients. Psychological Assessment, 10, 431-434.

Capps, L., Sigman, M., \& Yirmiya, N. (1995). Self-Competence and Emotional Understanding in High-Functioning Children with Autism. Development and Psychopathology, 7(1), 137-149.

Caron, M. J., Mottron, L., Berthiaume, C., \& Dawson, M. (2006). Cognitive mechanisms, specificity and neural underpinnings of visuospatial peaks in autism. Brain, 129(Pt 7), 1789-1802. doi: 10.1093/brain/awl072

Carper, R., \& Courchesne, E. (2000). Inverse correlation between frontal lobe and cerebellum sizes in children with autism. Brain, 123 (Pt 4), 836-844.

Casanova, M. F. (2004). White matter volume increase and minicolumns in autism. Ann Neurol, 56(3), 453; author reply 454. doi: 10.1002/ana.20196 
Casanova, M. F., Buxhoeveden, D., \& Gomez, J. (2003). Disruption in the inhibitory architecture of the cell minicolumn: implications for autism. [Research Support, Non-U.S. Gov't Research Support, U.S. Gov't, Non-P.H.S. Research Support, U.S. Gov't, P.H.S. Review]. Neuroscientist, 9(6), 496-507. doi: 10.1177/1073858403253552

Casanova, M. F., El-Baz, A. S., \& Suri, J. (2013). Imaging the brain in Autism: Springer.

Casanova, M. F., van Kooten, I. A., Switala, A. E., van Engeland, H., Heinsen, H., Steinbusch, H. W., . . Schmitz, C. (2006). Minicolumnar abnormalities in autism. Acta Neuropathol, 112(3), 287-303. doi: 10.1007/ s00401-006-0085-5

Chan, R. C., Shum, D., Toulopoulou, T., \& Chen, E. Y. (2008). Assessment of executive functions: review of instruments and identification of critical issues. [Research Support, Non-U.S. Gov't Review]. Arch Clin Neuropsychol, 23(2), 201-216. doi: 10.1016/j.acn.2007.08.010

Chandler, S., Charman, T., Baird, G., Simonoff, E., Loucas, T., Meldrum, D., . . Pickles, A. (2007). Validation of the social communication questionnaire in a population cohort of children with autism spectrum disorders. J Am Acad Child Adolesc Psychiatry, 46(10), 1324-1332. doi: 10.1097/chi.0b013e31812f7d8d

Chen, S. F., Chien, Y. L., Wu, C. T., Shang, C. Y., Wu, Y. Y., \& Gau, S. S. (2016). Deficits in executive functions among youths with autism spectrum disorders: an age-stratified analysis. Psycho/Med, 46(8), 1625-1638. doi: 10.1017/S0033291715002238

Cheng, Y., Chou, K. H., Chen, I. Y., Fan, Y. T., Decety, J., \& Lin, C. P. (2010). Atypical development of white matter microstructure in adolescents with autism spectrum disorders. Neuroimage, 50(3), 873-882. doi: 10.1016/j.neuroimage.2010.01.011

Cheng, Y., Chou, K. H., Fan, Y. T., \& Lin, C. P. (2011). ANS: aberrant neurodevelopment of the social cognition network in adolescents with autism spectrum disorders. PLoS One, 6(4), e18905. doi: 10.1371/journal. pone.0018905

Cherkassky, V. L., Kana, R. K., Keller, T. A., \& Just, M. A. (2006). Functional connectivity in a baseline resting-state network in autism. [Research Support, N.I.H., Extramural]. Neuroreport, 17(16), 1687-1690. doi: 10.1097/01.wnr.0000239956.45448.4C

Cochran, D. M., Sikoglu, E. M., Hodge, S. M., Edden, R. A., Foley, A., Kennedy, D. N., . . Frazier, J. A. (2015) Relationship among Glutamine, gamma-Aminobutyric Acid, and Social Cognition in Autism Spectrum Disorders. [Research Support, N.I.H., Extramural Research Support, Non-U.S. Gov't]. J Child Adolesc Psychopharmacol, 25(4), 314-322. doi: 10.1089/cap.2014.0112

Constantino, J. N., Davis, S. A., Todd, R. D., Schindler, M. K., Gross, M. M., Brophy, S. L., . . Reich, W. (2003). Validation of a brief quantitative measure of autistic traits: comparison of the social responsiveness scale with the autism diagnostic interview-revised. J Autism Dev Disord, 33(4), 427-433.

Corbett, B. A., Constantine, L. J., Hendren, R., Rocke, D., \& Ozonoff, S. (2009). Examining executive functioning in children with autism spectrum disorder, attention deficit hyperactivity disorder and typical development. Psychiatry Res, 166(2-3), 210-222.

Courchesne, E., Campbell, K., \& Solso, S. (2011). Brain growth across the life span in autism: age-specific changes in anatomical pathology. Brain Res, 1380, 138-145. doi: 10.1016/j.brainres.2010.09.101

Courchesne, E., Carper, R., \& Akshoomoff, N. (2003). Evidence of brain overgrowth in the first year of life in autism. JAMA, 290(3), 337-344. doi: 10.1001/jama.290.3.337

Courchesne, E., Karns, C. M., Davis, H. R., Ziccardi, R., Carper, R. A., Tigue, Z. D., . . Courchesne, R. Y. (2001) Unusual brain growth patterns in early life in patients with autistic disorder: an MRI study. Neurology, 57(2), 245-254.

Courchesne, E., \& Pierce, K. (2005). Why the frontal cortex in autism might be talking only to itself: local over-connectivity but long-distance disconnection. Curr Opin Neurobiol, 15(2), 225-230. doi: 10.1016/j. conb.2005.03.001

Craig, F., Margari, F., Legrottaglie, A. R., Palumbi, R., de Giambattista, C., \& Margari, L. (2016). A review of executive function deficits in autism spectrum disorder and attention-deficit/hyperactivity disorder. [Review]. Neuropsychiatr Dis Treat, 12, 1191-1202. doi: 10.2147/NDT.S104620

Crone, E. A., \& Dahl, R. E. (2012). Understanding adolescence as a period of social-affective engagement and goal flexibility. Nat Rev Neurosci, 13(9), 636-650. doi: 10.1038/nrn3313 
Crone, E. A., Wendelken, C., van Leijenhorst, L., Honomichl, R. D., Christoff, K., \& Bunge, S. A. (2009). Neurocognitive development of relational reasoning. Dev Sci, 12(1), 55-66. doi: 10.1111/j.1467-7687. 2008.00743.x

Crone, E. A., Zanolie, K., Van Leijenhorst, L., Westenberg, P. M., \& Rombouts, S. A. (2008). Neural mechanisms supporting flexible performance adjustment during development. Cogn Affect Behav Neurosci, 8(2), 165-177.

Dahlgren, S. O., \& Trillingsgaard, A. (1996). Theory of mind in non-retarded children with autism and Asperger's syndrome. A research note. [Comparative Study Research Support, Non-U.S. Gov't]. J Child Psychol Psychiatry, 37(6), 759-763.

Dapretto, M., Davies, M. S., Pfeifer, J. H., Scott, A. A., Sigman, M., Bookheimer, S. Y., \& lacoboni, M. (2006). Understanding emotions in others: mirror neuron dysfunction in children with autism spectrum disorders. [Clinical Trial Research Support, N.I.H., Extramural Research Support, Non-U.S. Gov't]. Nat Neurosci, 9(1), 28-30. doi: 10.1038/nn1611

David, N., Aumann, C., Bewernick, B. H., Santos, N. S., Lehnhardt, F. G., \& Vogeley, K. (2010). Investigation of mentalizing and visuospatial perspective taking for self and other in Asperger syndrome. [Research Support, Non-U.S. Gov't]. J Autism Dev Disord, 40(3), 290-299. doi: 10.1007/s10803-009-0867-4

David, O., Guillemain, I., Saillet, S., Reyt, S., Deransart, C., Segebarth, C., \& Depaulis, A. (2008). Identifying neural drivers with functional MRI: an electrophysiological validation. [Evaluation Studies Research Support, Non-U.S. Gov't]. PLoS Biol, 6(12), 2683-2697. doi: 10.1371/journal.pbio.0060315

Dawson, G., Webb, S., Schellenberg, G., Dager, S., Friedman, S., Aylward, E., \& Richards, T. (2002). Defining the broader phenotype of autism: genetic, brain, and behavioral perspectives. Dev Psychopathol, 14(3), 581-611.

de Bildt, A., Sytema, S., van Lang, N. D., Minderaa, R. B., van Engeland, H., \& de Jonge, M. V. (2009). Evaluation of the ADOS revised algorithm: the applicability in 558 Dutch children and adolescents. [Research Support, Non-U.S. Gov't]. J Autism Dev Disord, 39(9), 1350-1358. doi: 10.1007/s10803-009-0749-9

de Graaf, P., Goricke, S., Rodjan, F., Galluzzi, P., Maeder, P., Castelijns, J. A., \& Brisse, H. J. (2012). Guidelines for imaging retinoblastoma: imaging principles and MRI standardization. [Practice Guideline]. Pediatr Radiol, 42(1), 2-14. doi: 10.1007/s00247-011-2201-5

De Los Reyes, A. (2011). Internal Consistency and Associated Characteristics of Informant Discrepancies in Clinic Referred Youths Age 11 to 17 Years. Journal of Clinical Child \& Adolescent Psychology, 40(1), 36-53.

Delis, D. C., Kaplan, E., \& Kramer, J. H. (2007). Tower Test (I. Noens \& I. v. d. Berckelaer-Onnes, Trans.) Delis - Kaplan Executive Function System Manual. London: Harcourt Test Publishers.

Delis, D. C., Kaplan, E., \& Kramer, J. H. (2008). Design Fluency Test (I. Noens \& I. v. d. Berckelaer-Onnes, Trans.) Delis - Kaplan Executive Function System Manual. London: Harcourt Test Publishers.

Dennis, M., Agostino, A., Roncadin, C., \& Levin, H. (2009). Theory of mind depends on domain-general executive functions of working memory and cognitive inhibition in children with traumatic brain injury. $J$ Clin Exp Neuropsychol, 31(7), 835-847. doi: 10.1080/13803390802572419

Dennis, M., Francis, D. J., Cirino, P. T., Schachar, R., Barnes, M. A., \& Fletcher, J. M. (2009). Why IQ is not a covariate in cognitive studies of neurodevelopmental disorders. [Research Support, N.I.H., Extramural Review]. J Int Neuropsycho/ Soc, 15(3), 331-343. doi: 10.1017/S1355617709090481

Deshpande, G., LaConte, S., James, G. A., Peltier, S., \& Hu, X. (2009). Multivariate Granger causality analysis of fMRI data. [Research Support, N.I.H., Extramural Research Support, Non-U.S. Gov't]. Hum Brain Mapp, 30(4), 1361-1373. doi: 10.1002/hbm.20606

Deshpande, G., Santhanam, P., \& Hu, X. (2011). Instantaneous and causal connectivity in resting state brain networks derived from functional MRI data. [Research Support, N.I.H., Extramural Research Support, Non-U.S. Gov't]. Neuroimage, 54(2), 1043-1052. doi: 10.1016/j.neuroimage.2010.09.024

Dhossche, D., Applegate, H., Abraham, A., Maertens, P., Bland, L., Bencsath, A., \& Martinez, J. (2002). Elevated plasma gamma-aminobutyric acid (GABA) levels in autistic youngsters: stimulus for a GABA hypothesis of autism. [Research Support, Non-U.S. Gov't]. Med Sci Monit, 8(8), PR1-6.

Diagnostic and Statistical Manual of Mental Disorders, fourth ed. (1994). (fourth ed.). Washington DC.: American Psychiatry Association. 
Donaldson, T. S. (1968). Robustness of the F-test to errors of both kinds and the correlation between the numerator and denominator of the F-ratio. Journal of the American Statistical Association(63), 660-676.

Donders, J. (1997). A short form of the WISC-III for clinical use. Psychological Assessment, 9, 15-20.

Donders, J. (2001). Using a short form of the WISC-III: sinful or smart? Child Neuropsychol, 7(2), 99-103.

Donnellan, A. M., Leary, M. R., \& Patterson-Robledo, J. (2006). I Can't Get Started, Stress and the Role of Movement Differences in People with Autism. In M. G. Baron, J. Groden, G. Groden \& L. P. Lipsitt (Eds.), Stress and Coping in Autism (pp. 204-245). Oxford: Oxford University Press.

Drenthen, G. S., Barendse, E. M., Aldenkamp, A. P., van Veenendaal, T. M., Puts, N. A., Edden, R. A., . . Jansen, J. F. (2016). Altered neurotransmitter metabolism in adolescents with high-functioning autism. Psychiatry Res, 256, 44-49. doi: 10.1016/j.pscychresns.2016.09.007

Dumontheil, I., Apperly, I. A., \& Blakemore, S. J. (2010). Online usage of theory of mind continues to develop in late adolescence. Dev Sci, 13(2), 331-338. doi: 10.1111/j.1467-7687.2009.00888.x

Dunn, O. J., \& Clark, V. A. (1969). Correlational coefficients measured on the same individuals. Journal of the American Statistical Association, 64, 366-377.

Ebesutani, C., Bernstein, A., Martinez, J. I., Chorpita, B. F., \& Weisz, J. R. (2011). The youth self report: applicability and validity across younger and older youths. J Clin Child Adolesc Psychol, 40(2), 338-346 doi: 10.1080/15374416.2011.546041

Ebesutani, C., Bernstein, A., Nakamura, B. J., Chorpita, B. F., Higa-McMillan, C. K., Weisz, J. R., \& Health, T. R. N. O. Y. M. (2010). Concurrent Validity of the Child Behavior Checklist DSM-Oriented Scales: Correspondence with DSM Diagnoses and Comparison to Syndrome Scales. J Psychopathol Behav Assess, 32(3), 373-384. doi: 10.1007/s10862-009-9174-9

Edden, R. A., Puts, N. A., Harris, A. D., Barker, P. B., \& Evans, C. J. (2014). Gannet: A batch-processing tool for the quantitative analysis of gamma-aminobutyric acid-edited MR spectroscopy spectra. [Research Support, N.I.H., Extramural]. J Magn Reson Imaging, 40(6), 1445-1452. doi: 10.1002/jmri.24478

Ehlers, S., Gillberg, C., \& Wing, L. (1999). A screening questionnaire for Asperger syndrome and other high-functioning autism spectrum disorders in school age children. J Autism Dev Disord, 29(2), 129-141.

Eigsti, I. M. (2011). Executive functions in ASD. In D. Fein (Ed.), The neuropsychology of autism (pp. 185-203). New York: Oxford University Press.

Ekman, P. (1992). An Argument for Basic Emotions. Cognition \& Emotion, 6(3-4), 169-200. doi: Doi 10.1080/02699939208411068

Ekman, P., \& Friesen, W. V. (1971). Constants across cultures in the face and emotion. J Pers Soc Psychol, 17(2), 124-129.

El-Ansary, A., \& Al-Ayadhi, L. (2014). GABAergic/glutamatergic imbalance relative to excessive neuroinflammation in autism spectrum disorders. [Research Support, Non-U.S. Gov't]. J Neuroinflammation, 11, 189 doi: 10.1186/s12974-014-0189-0

Elsabbagh, M., Divan, G., Koh, Y. J., Kim, Y. S., Kauchali, S., Marcin, C., . . Fombonne, E. (2012). Global prevalence of autism and other pervasive developmental disorders. Autism Res, 5(3), 160-179. doi: 10.1002/aur.239

Evers, A., Vliet-Mulder,J.C. van, \& Groot, C.J. (2007). Documentatie van tests en testresearch in Nederland, aanvulling 2007/01 (COTAN). Amsterdam: Boom test uitgevers.

Evers, M., \& Hollander, E. (2008). Excitotoxocoty in Autism. In A. W. Zimmerman (Ed.), Autism (pp. 133-145). Totowa, NJ: Humana Press.

Fatemi, S. H., Reutiman, T. J., Folsom, T. D., \& Thuras, P. D. (2009). GABA(A) receptor downregulation in brains of subjects with autism. [Research Support, N.I.H., Extramural Research Support, Non-U.S. Gov't]. J Autism Dev Disord, 39(2), 223-230. doi: 10.1007/s10803-008-0646-7

Ferdinand, R. F. (2008). Validity of the CBCL/YSR DSM-IV scales Anxiety Problems and Affective Problems. J Anxiety Disord, 22(1), 126-134. doi: 10.1016/j.janxdis.2007.01.008

Field, A. (2009). Analysis of covariance, ANCOVA (GLM2) Discovering Statistics Using SPSS (3 ed., pp. 397-399). London: SAGE Publications Ltd.

Filippini, N., Maclntosh, B. J., Hough, M. G., Goodwin, G. M., Frisoni, G. B., Smith, S. M., . . Mackay, C. E. (2009). Distinct patterns of brain activity in young carriers of the APOE-epsilon4 allele. [Research Support, Non-U.S. Gov't]. Proc Natl Acad Sci U S A, 106(17), 7209-7214. doi: 10.1073/pnas.0811879106 
Finn, A. S., Sheridan, M. A., Kam, C. L., Hinshaw, S., \& D'Esposito, M. (2010). Longitudinal evidence for functional specialization of the neural circuit supporting working memory in the human brain. J Neurosci, 30(33), 11062-11067. doi: 10.1523/JNEUROSCI.6266-09.2010

Frangou, S., Chitins, X., \& Williams, S. C. (2004). Mapping IQ and gray matter density in healthy young people. Neuroimage, 23(3), 800-805. doi: 10.1016/j.neuroimage.2004.05.027

Freitag, C. M., Luders, E., Hulst, H. E., Narr, K. L., Thompson, P. M., Toga, A. W., . . Konrad, C. (2009). Total brain volume and corpus callosum size in medication-naïve adolescents and young adults with autism spectrum disorder. Biol Psychiatry, 66(4), 316-319. doi: 10.1016/j.biopsych.2009.03.011

Friedman, N. P., Miyake, A., Corley, R. P., Young, S. E., Defries, J. C., \& Hewitt, J. K. (2006). Not all executive functions are related to intelligence. [Research Support, N.I.H., Extramural]. Psychol Sci, 17(2), 172-179. doi: 10.1111/j.1467-9280.2006.01681.x

Frith, U. (1989). Autism: Explaining the enigma. Oxford: Basil Blackwell.

Gaetz, W., Bloy, L., Wang, D. J., Port, R. G., Blaskey, L., Levy, S. E., \& Roberts, T. P. (2014). GABA estimation in the brains of children on the autism spectrum: measurement precision and regional cortical variation. [Research Support, N.I.H., Extramural Research Support, Non-U.S. Gov't Review]. Neuroimage, 86, 1-9. doi: 10.1016/j.neuroimage.2013.05.068

Geschwind, D. H. (2011). Genetics of autism spectrum disorders. Trends Cogn Sci, 15(9), 409-416. doi: 10.1016/j.tics.2011.07.003

Geurts, H. M., Verté, S., Oosterlaan, J., Roeyers, H., \& Sergeant, J. (2004). How specific are executive functioning deficits in attention deficit hyperactivity disorder and autism? J Child Psychol Psychiatry, 45(4), 836-854. doi: 10.1111/j.1469-7610.2004.00276.x

Geurts, H. M., \& Vissers, M. E. (2012). Elderly with autism: executive functions and memory. J Autism Dev Disord, 42(5), 665-675. doi: 10.1007/s10803-011-1291-0

Geweke, J. F. (1984). Measures of Conditional Linear Dependence and Feedback between Time Series. Journal of the American Statistical Association, 79(388), 907-915.

Giedd, J. (2008). The teen brain: insights from neuroimaging. J Adolesc Health, 42(4), 335-343. doi: 1016/j. jadohealth.2008.01.007

Gilbert, S. J., Bird, G., Brindley, R., Frith, C. D., \& Burgess, P. W. (2008). Atypical recruitment of medial prefrontal cortex in autism spectrum disorders: an fMRI study of two executive function tasks. Neuropsychologia, 46(9), 2281-2291. doi: 10.1016/j.neuropsychologia.2008.03.025

Gilotty, L., Kenworthy, L., Sirian, L., Black, D. O., \& Wagner, A. E. (2002). Adaptive skills and executive function in autism spectrum disorders. Child Neuropsychol, 8(4), 241-248.

Gioia, G. A., Isquith, P. K., Guy, S. C., \& Kenworthy, L. (2009). BRIEF Executieve Functies Gedragsvragenlijst Handleiding (D. Smidts \& M. Huizinga, Trans.). Amsterdam: Hogrefe Uitgevers B.V.

Goldberg, M. C., Mostofsky, S. H., Cutting, L. E., Mahone, E. M., Astor, B. C., Denckla, M. B., \& Landa, R. J. (2005). Subtle executive impairment in children with autism and children with ADHD. J Autism Dev Disord, 35(3), 279-293.

Granader, Y., Wallace, G. L., Hardy, K. K., Yerys, B. E., Lawson, R. A., Rosenthal, M., . . Kenworthy, L. (2014). Characterizing the factor structure of parent reported executive function in autism spectrum disorders: the impact of cognitive inflexibility. [Research Support, N.I.H., Extramural Research Support, Non-U.S. Gov't]. J Autism Dev Disord, 44(12), 3056-3062. doi: 10.1007/s10803-014-2169-8

Granger, C. W. J. (1969). Investigating Causal Relations by Econometric Models and Cross-spectral Methods Econometrica, 37(3), 424-438 doi: 10.2307/1912791

Gray, J. R., Chabris, C. F., \& Braver, T. S. (2003). Neural mechanisms of general fluid intelligence. Nat Neurosci, 6(3), 316-322. doi: 10.1038/nn1014

Greicius, M. D., Srivastava, G., Reiss, A. L., \& Menon, V. (2004). Default-mode network activity distinguishes Alzheimer's disease from healthy aging: evidence from functional MRI. [Research Support, Non-U.S. Gov't Research Support, U.S. Gov't, P.H.S.]. Proc Natl Acad Sci U S A, 101(13), 4637-4642. doi: 10.1073/ pnas.0308627101

Greimel, E., Schulte-Ruther, M., Kamp-Becker, I., Remschmidt, H., Herpertz-Dahlmann, B., \& Konrad, K. (2014). Impairment in face processing in autism spectrum disorder: a developmental perspective. J Neural Transm (Vienna), 121(9), 1171-1181. doi: 10.1007/s00702-014-1206-2 
Groen, W. B., Buitelaar, J. K., van der Gaag, R. J., \& Zwiers, M. P. (2011). Pervasive microstructural abnormalities in autism: a DTI study. J Psychiatry Neurosci, 36(1), 32-40. doi: 10.1503/jpn.090100

Grossman, J. B., Klin, A., Carter, A. S., \& Volkmar, F. R. (2000). Verbal bias in recognition of facial emotions in children with Asperger syndrome. [Research Support, U.S. Gov't, P.H.S.]. J Child Psychol Psychiatry, 41(3), 369-379.

Hadjikhani, N., Joseph, R. M., Snyder, J., \& Tager-Flusberg, H. (2006). Anatomical differences in the mirror neuron system and social cognition network in autism. Cereb Cortex, 16(9), 1276-1282. doi: 10.1093/ cercor/bhj069

Hamilton, A. F. (2013). Reflecting on the mirror neuron system in autism: a systematic review of current theories. Dev Cogn Neurosci, 3, 91-105. doi: 10.1016/j.dcn.2012.09.008

Happé, F., Booth, R., Charlton, R., \& Hughes, C. (2006). Executive function deficits in autism spectrum disorders and attention-deficit/hyperactivity disorder: examining profiles across domains and ages. Brain Cogn, 61(1), 25-39. doi: 10.1016/j.bandc.2006.03.004

Happe, F., \& Frith, U. (1996). The neuropsychology of autism. Brain, 119 ( Pt 4), 1377-1400.

Happe, F., \& Ronald, A. (2008). The 'fractionable autism triad': a review of evidence from behavioural, genetic, cognitive and neural research. Neuropsychol Rev, 18(4), 287-304. doi: 10.1007/s11065-008-9076-8

Happe, F. G. (1994a). An advanced test of theory of mind: understanding of story characters' thoughts and feelings by able autistic, mentally handicapped, and normal children and adults. J Autism Dev Disord, 24(2), 129-154.

Happe, F. G. (1994b). Annotation: current psychological theories of autism: the "theory of mind" account and rival theories. J Child Psychol Psychiatry, 35(2), 215-229.

Harada, M., Taki, M. M., Nose, A., Kubo, H., Mori, K., Nishitani, H., \& Matsuda, T. (2011). Non-invasive evaluation of the GABAergic/glutamatergic system in autistic patients observed by MEGA-editing proton MR spectroscopy using a clinical 3 tesla instrument. [Research Support, Non-U.S. Gov't]. J Autism Dev Disord, 41(4), 447-454. doi: 10.1007/s10803-010-1065-0

Hardan, A., Muddasani, S., Vemulapalli, M., Keshavan, M., \& Minshew, N. (2006). An MRI study of increased cortical thickness in autism. Am J Psychiatry, 163(7), 1290-1292. doi: 10.1176/appi.ajp.163.7.1290

Harms, M. B., Martin, A., \& Wallace, G. L. (2010). Facial emotion recognition in autism spectrum disorders: a review of behavioral and neuroimaging studies. Neuropsychol Rev, 20(3), 290-322. doi: 10.1007/s11065010-9138-6

Hauck, M., Fein, D., Waterhouse, L., \& Feinstein, C. (1995). Social initiations by autistic children to adults and other children. [Comparative Study Research Support, U.S. Gov't, P.H.S.]. J Autism Dev Disord, 25(6), 579-595.

Herbert, M. R., Ziegler, D. A., Deutsch, C. K., O'Brien, L. M., Lange, N., Bakardjiev, A., . . Caviness, V. S. J. (2003). Dissociations of cerebral cortex, subcortical and cerebral white matter volumes in autistic boys. Brain, 126(Pt 5), 1182-1192.

Hill, E. L. (2004). Executive dysfunction in autism. Trends Cogn Sci, 8(1), 26-32. doi: S1364661303003152 [pii]

Hill, E. L., \& Frith, U. (2003). Understanding autism: insights from mind and brain. Philos Trans R Soc Lond B Biol Sci, 358(1430), 281-289. doi: 10.1098/rstb.2002.1209

Hofmann, W., Schmeichel, B. J., \& Baddeley, A. D. (2012). Executive functions and self-regulation. [Review]. Trends Cogn Sci, 16(3), 174-180. doi: 10.1016/j.tics.2012.01.006

Huizinga, M., Dolan, C. V., \& van der Molen, M. W. (2006). Age-related change in executive function: developmental trends and a latent variable analysis. Neuropsychologia, 44(11), 2017-2036. doi: S00283932(06)00022-4 [pii] 10.1016/j.neuropsychologia.2006.01.010

Hurtig, T., Kuusikko, S., Mattila, M. L., Haapsamo, H., Ebeling, H., Jussila, K., . . Moilanen, I. (2009). Multi-informant reports of psychiatric symptoms among high-functioning adolescents with Asperger syndrome or autism. [Research Support, Non-U.S. Gov't]. Autism, 13(6), 583-598. doi: 10.1177/1362361309335719

Hyvarinen, A. (1999). Fast and robust fixed-point algorithms for independent component analysis. IEEE Trans Neural Netw, 10(3), 626-634. doi: 10.1109/72.761722

James, I. A., Mukaetova-Ladinska, E., Reichelt, F. K., Briel, R., \& Scully, A. (2006). Diagnosing Aspergers syndrome in the elderly: a series of case presentations. [Case Reports]. Int J Geriatr Psychiatry, 21(10), 951-960. doi: 10.1002/gps.1588 
Jarrold, C., \& Towse, J. N. (2006). Individual differences in working memory. Neuroscience, 139(1), 39-50. doi: 10.1016/j.neuroscience.2005.07.002

Jenkinson, M., Bannister, P., Brady, M., \& Smith, S. (2002). Improved optimization for the robust and accurate linear registration and motion correction of brain images. [Research Support, Non-U.S. Gov't]. Neuroimage, 17(2), 825-841.

Johnson, K. A., Robertson, I. H., Kelly, S. P., Silk, T. J., Barry, E., Daibhis, A., . . Bellgrove, M. A. (2007). Dissociation in performance of children with ADHD and high-functioning autism on a task of sustained attention. [Research Support, Non-U.S. Gov't]. Neuropsychologia, 45(10), 2234-2245. doi: 10.1016/j. neuropsychologia.2007.02.019

Johnson, S. A., Filliter, J. H., \& Murphy, R. R. (2009). Discrepancies Between Self- and Parent-Perceptions of Autistic Traits and Empathy in High Functioning Children and Adolescents on the Autism Spectrum. J Autism Dev Disord. doi: 10.1007/s10803-009-0809-1

Jones, C. R., Pickles, A., Falcaro, M., Marsden, A. J., Happe, F., Scott, S. K., . . Charman, T. (2011). A multimodal approach to emotion recognition ability in autism spectrum disorders. J Child Psychol Psychiatry, 52(3), 275-285. doi: 10.1111/j.1469-7610.2010.02328.x

Jones, T. B., Bandettini, P. A., Kenworthy, L., Case, L. K., Milleville, S. C., Martin, A., \& Birn, R. M. (2010). Sources of group differences in functional connectivity: an investigation applied to autism spectrum disorder. Neuroimage, 49(1), 401-414. doi: 10.1016/j.neuroimage.2009.07.051

Jong de, P. F. (2006). Understanding normal and impaired reading development: a working memory perspective. In S. J. Pickering (Ed.), Working memory and education: Academic Press Elsevier Inc.

Joseph, R. M. (2011). The significance of IQ and differential cognitive abilities for understading ASD. In D. Fein (Ed.), The neuropsychology of autism (pp. 281-294). New York: Oxford University Press.

Joshi, G., Biederman, J., Wozniak, J., Goldin, R. L., Crowley, D., Furtak, S., . . Gonenc, A. (2013). Magnetic resonance spectroscopy study of the glutamatergic system in adolescent males with high-functioning autistic disorder: a pilot study at 4T. [Research Support, Non-U.S. Gov't]. Eur Arch Psychiatry Clin Neurosci, 263(5), 379-384. doi: 10.1007/s00406-012-0369-9

Just, M. A., Cherkassky, V. L., Keller, T. A., \& Minshew, N. J. (2004). Cortical activation and synchronization during sentence comprehension in high-functioning autism: evidence of underconnectivity. Brain, 127(Pt 8), 1811-1821. doi: 10.1093/brain/awh199

Just, M. A., Keller, T. A., Malave, V. L., Kana, R. K., \& Varma, S. (2012). Autism as a neural systems disorder: a theory of frontal-posterior underconnectivity. Neurosci Biobehav Rev, 36(4), 1292-1313. doi: 10.1016/j. neubiorev.2012.02.007

Kaiser, S., Kopka, M. L., Rentrop, M., Walther, S., Kronmüller, K., Olbrich, R., . . Stippich, C. (2010). Maintenance of real objects and their verbal designations in working memory. Neurosci Lett, 469(1), 65-69. doi: 10.1016/j.neulet.2009.11.045

Kaland, N., Callesen, K., Moller-Nielsen, A., Mortensen, E. L., \& Smith, L. (2008). Performance of children and adolescents with Asperger syndrome or high-functioning autism on advanced theory of mind tasks. $J$ Autism Dev Disord, 38(6), 1112-1123. doi: 10.1007/s10803-007-0496-8

Kaland, N., Moller-Nielsen, A., Callesen, K., Mortensen, E. L., Gottlieb, D., \& Smith, L. (2002). A new 'advanced' test of theory of mind: evidence from children and adolescents with Asperger syndrome. J Child Psychol Psychiatry, 43(4), 517-528.

Kaland, N., Smith, L., \& Mortensen, E. L. (2007). Response times of children and adolescents with Asperger syndrome on an 'advanced' test of theory of mind. J Autism Dev Disord, 37(2), 197-209. doi: 10.1007/ s10803-006-0152-8

Kana, R. K., Keller, T. A., Minshew, N. J., \& Just, M. A. (2007). Inhibitory control in high-functioning autism: decreased activation and underconnectivity in inhibition networks. Biol Psychiatry, 62(3), 198-206. doi: 10.1016/j.biopsych.2006.08.004

Kane, M. J., Hambrick, D. Z., Tuholski, S. W., Wilhelm, O., Payne, T. W., \& Engle, R. W. (2004). The generality of working memory capacity: a latent-variable approach to verbal and visuospatial memory span and reasoning. J Exp Psychol Gen, 133(2), 189-217. doi: 10.1037/0096-3445.133.2.189

Kanner, L. (1943). Autistic disturbances of affective contact. The nervous child: quaterly journal of psychopathology, psychotherapy, mental hygiene, and guidance of the child, 217-250. 
Kanner, L. (1965). Infantile autism and the schizophrenias. Behav Sci, 10(4), 412-420.

Kelley, E. (2011). Language in ASD. In D. Fein (Ed.), The neuropsychology of autism (pp. 123-137). New York: Oxford University Press.

Kenet, T. (2011). Sensory functions in ASD. In D. Fein (Ed.), The neuropsychology of autism (pp. 215-224). New York: Oxford University Press.

Kessels, R. S., P; Hendriks, AW. (2010). Perception of dynamic facial emotional expressions in adolescents with autism spectrum disorders (ASD). Translational Neuroscience, 1(3), 228-232. doi: 10.2478/v10134010-0033-8

Klinger, L. G., Klinger, M. R., \& Pohlig, R. L. (2007). Implicit Learning Impairments in Autism Spectrum Disorders. In J. M. Pérez, P. M. González, M. L. Comí \& C. Nieto (Eds.), New Developments in Autism: The Future is Today (pp. 76-103). London, UK: Jessica Kingsley.

Koch, K., Wagner, G., von Consbruch, K., Nenadic, I., Schultz, C., Ehle, C., . . Schlosser, R. (2006). Temporal changes in neural activation during practice of information retrieval from short-term memory: an fMRI study. Brain Res, 1107(1), 140-150. doi: 10.1016/j.brainres.2006.06.003

Koning, C., \& Magill-Evans, J. (2001). Social and language skills in adolescent boys with Asperger syndrome. [Research Support, Non-U.S. Gov't]. Autism, 5(1), 23-36.

Koshino, H., Kana, R. K., Keller, T. A., Cherkassky, V. L., Minshew, N. J., \& Just, M. A. (2008). fMRI investigation of working memory for faces in autism: visual coding and underconnectivity with frontal areas. Cereb Cortex, 18(2), 289-300. doi: 10.1093/cercor/bhm054

Kubas, B., Kulak, W., Sobaniec, W., Tarasow, E., Lebkowska, U., \& Walecki, J. (2012). Metabolite alterations in autistic children: a 1H MR spectroscopy study. Adv Med Sci, 57(1), 152-156. doi: 10.2478/v10039-012-0014-x

Kuo, M. H., Orsmond, G. I., Cohn, E. S., \& Coster, W. J. (2013). Friendship characteristics and activity patterns of adolescents with an autism spectrum disorder. [Research Support, Non-U.S. Gov't]. Autism, 17(4), 481-500. doi: 10.1177/1362361311416380

Kuusikko, S., Haapsamo, H., Jansson-Verkasalo, E., Hurtig, T., Mattila, M. L., Ebeling, H., . . Moilanen, I. (2009). Emotion recognition in children and adolescents with autism spectrum disorders. J Autism Dev Disord, 39(6), 938-945. doi: 10.1007/s10803-009-0700-0

Kuusikko, S., Pollock-Wurman, R., Jussila, K., Carter, A. S., Mattila, M. L., Ebeling, H., . . Moilanen, I. (2008). Social anxiety in high-functioning children and adolescents with Autism and Asperger syndrome. $J$ Autism Dev Disord, 38(9), 1697-1709. doi: 10.1007/s10803-008-0555-9

Lahuis, B. E., Durston, S., Nederveen, H., Zeegers, M., Palmen, S. J., \& Van Engeland, H. (2008). MRI-based morphometry in children with multiple complex developmental disorder, a phenotypically defined subtype of pervasive developmental disorder not otherwise specified. Psychol Med, 38(9), 1361-1367. doi: 10.1017/S0033291707001481

Lainhart, J. E., Cooperrider, J., \& Taylor, J. S. (2013). Spectoscopic Brain Imaging in Autism. In M. F. Casanova, A. S. El-Baz \& J. S. Suri (Eds.), Imaging the Brain in Autism (pp. pp. 231-288). New York NY: Springer New York.

Lam, K., Aman, M., \& Arnold, L. (2006). Neurochemical correlates of autistic disorder: a review of the literature. Res Dev Disabil, 27(3), 254-289. doi: 10.1016/j.ridd.2005.03.003

Landa, R. J., \& Goldberg, M. C. (2005). Language, social, and executive functions in high functioning autism: a continuum of performance. J Autism Dev Disord, 35(5), 557-573. doi: 10.1007/s10803-005-0001-1

Langen, M., Durston, S., Staal, W. G., Palmen, S. J., \& van Engeland, H. (2007). Caudate nucleus is enlarged in high-functioning medication-naive subjects with autism. Biol Psychiatry, 62(3), 262-266. doi: 10.1016/j. biopsych.2006.09.040

Langen, M., Schnack, H., Nederveen, H., Bos, D., Lahuis, B., de Jonge, M., .. . Durston, S. (2009). Changes in the developmental trajectories of striatum in autism. Biol Psychiatry, 66(4), 327-333. doi: 10.1016/j. biopsych.2009.03.017

Langner, O., Dotsch, R., Bijlstra, G., Wigboldus, D. H. J., Hawk, S. T., \& van Knippenberg, A. (2010). Presentation and validation of the Radboud Faces Database. Cognition \& Emotion, 24(8), 1377-1388. doi: Pii 930020275 Doi 10.1080/02699930903485076

Larochette, A. C., Benn, K., \& Harrison, A. G. (2009). Executive functioning: a comparison of the Tower of London(DX)andtheD-KEFSTowerTest.App/Neuropsychol, 16(4),275-280.doi: 10.1080/09084280903098695 
Lawson, R. A., Papadakis, A. A., Higginson, C. I., Barnett, J. E., Wills, M. C., Strang, J. F., . . Kenworthy, L. (2015). Everyday executive function impairments predict comorbid psychopathology in autism spectrum and attention deficit hyperactivity disorders. [Research Support, Non-U.S. Gov't]. Neuropsychology, 29(3), 445-453. doi: 10.1037/neu0000145

Leon-Carrion, J., Izzetoglu, M., Izzetoglu, K., Martin-Rodriguez, J. F., Damas-Lopez, J., Barroso y Martin, J. M., \& Dominguez-Morales, M. R. (2010). Efficient learning produces spontaneous neural repetition suppression in prefrontal cortex. Behav Brain Res, 208(2), 502-508. doi: 10.1016/j.bbr.2009.12.026

Lerner, M. D., Calhoun, C. D., Mikami, A. Y., \& De Los Reyes, A. (2012). Understanding parent-child social informant discrepancy in youth with high functioning autism spectrum disorders. J Autism Dev Disord, 42(12), 2680-2692. doi: 10.1007/s10803-012-1525-9

Leung, R. C., Vogan, V. M., Powell, T. L., Anagnostou, E., \& Taylor, M. J. (2016). The role of executive functions in social impairment in Autism Spectrum Disorder. [Research Support, Non-U.S. Gov't]. Child Neuropsychol, 22(3), 336-344. doi: 10.1080/09297049.2015.1005066

Levy, S. E., Mandell, D. S., \& Schultz, R. T. (2009). Autism. Lancet, 374(9701), 1627-1638.

Lezak, M. D., Howieson, D. B., Bigler, E. D., \& Tranel, D. (2012). Neuropsychological Assessment (5th ed.). New York: Oxford University Press.

Liao, W., Mantini, D., Zhang, Z., Pan, Z., Ding, J., Gong, Q., . . . Chen, H. (2010). Evaluating the effective connectivity of resting state networks using conditional Granger causality. Biol Cybern, 102(1), 57-69. doi: 10.1007/s00422-009-0350-5

Linden, D. E. (2007). The working memory networks of the human brain. Neuroscientist, 13(3), 257-267. doi: $10.1177 / 1073858406298480$

Liss, M., Fein, D., Allen, D., Dunn, M., Feinstein, C., Morris, R., . . Rapin, I. (2001). Executive functioning in high-functioning children with autism. J Child Psychol Psychiatry, 42(2), 261-270.

LoPresti, M. L., Schon, K., Tricarico, M. D., Swisher, J. D., Celone, K. A., \& Stern, C. E. (2008). Working memory for social cues recruits orbitofrontal cortex and amygdala: a functional magnetic resonance imaging study of delayed matching to sample for emotional expressions. [Research Support, N.I.H., Extramural Research Support, Non-U.S. Gov't Research Support, U.S. Gov't, Non-P.H.S.]. J Neurosci, 28(14), 3718-3728. doi: 10.1523/JNEUROSCI.0464-08.2008

Lord, C., Risi, S., Lambrecht, L., Cook, E. H., Leventhal, B. L., DiLavore, P. C., . . Rutter, M. (2000). The Autism Diagnostic Observation Schedule-Generic: A standard measure of social and communication deficits associated with the spectrum of autism. J Autism Dev Disord, 30(3), 205-223. doi: Doi 10.1023/A:1005592401947

Lord, C., Rutter, M., DiLavore, P. C., \& Risi, S. (2010). ADOS Autisme Diagnostisch Observatie Schema Handleiding (A. d. Bildt \& M. d. Jonge, Trans.). Amsterdam: Hogrefe Uitgevers B.V.

Loveland, K. A., Tunali-Kotoski, B., Chen, Y. R., Ortegon, J., Pearson, D. A., Brelsford, K. A., \& Gibbs, M. C. (1997). Emotion recognition in autism: verbal and nonverbal information. Dev Psychopathol, 9(3), 579-593.

Lowe, M. J., Mock, B. J., \& Sorenson, J. A. (1998). Functional connectivity in single and multislice echoplanar imaging using resting-state fluctuations. [Clinical Trial

Research Support, Non-U.S. Gov't Research Support, U.S. Gov't, P.H.S.]. Neuroimage, 7(2), 119-132. doi: 10.1006/nimg.1997.0315

Luna, B., Doll, S. K., Hegedus, S. J., Minshew, N. J., \& Sweeney, J. A. (2007). Maturation of executive function in autism. Biol Psychiatry, 61(4), 474-481. doi: 10.1016/j.biopsych.2006.02.030

Luna, B., Minshew, N. J., Garver, K. E., Lazar, N. A., Thulborn, K. R., Eddy, W. F., \& Sweeney, J. A. (2002). Neocortical system abnormalities in autism: an fMRI study of spatial working memory. Neurology, 59(6), 834-840.

Mahone, E. M., Hagelthorn, K. M., Cutting, L. E., Schuerholz, L. J., Pelletier, S. F., Rawlins, C., . . Denckla, M. B. (2002). Effects of IQ on executive function measures in children with ADHD. Child Neuropsychol, 8(1), 52-65. doi: 10.1076/chin.8.1.52.8719

Manly, T., Robertson, I. H., Anderson, V., \& Nimmo-Smith, I. (2004). Test of Everyday Attention for Children Manual (H. Assessment, Trans.): Harcourt Assessment. 
Mazefsky, C. A., Anderson, R., Conner, C. M., \& Minshew, N. (2011). Child Behavior Checklist Scores for School-Aged Children with Autism: Preliminary Evidence of Patterns Suggesting the Need for Referral. J Psychopathol Behav Assess, 33(1), 31-37. doi: 10.1007/s10862-010-9198-1

Mazefsky, C. A., Kao, J., \& Oswald, D. P. (2011). Preliminary evidence suggesting caution in the use of psychiatric self-report measures with adolescents with high-functioning autism spectrum disorders. Res Autism Spectr Disord, 5(1), 164-174. doi: 10.1016/j.rasd.2010.03.006

Mazurek, M. O., \& Kanne, S. M. (2010). Friendship and internalizing symptoms among children and adolescents with ASD. [Research Support, Non-U.S. Gov't]. J Autism Dev Disord, 40(12), 1512-1520. doi: 10.1007/ s10803-010-1014-y

Mazza, M., Pino, M. C., Mariano, M., Tempesta, D., Ferrara, M., De Berardis, D., . . Valenti, M. (2014). Affective and cognitive empathy in adolescents with autism spectrum disorder. Front Hum Neurosci, 8, 791. doi: 10.3389/fnhum.2014.00791

McAlonan, G. M., Cheung, V., Cheung, C., Suckling, J., Lam, G. Y., Tai, K. S., . . Chua, S. E. (2005). Mapping the brain in autism. A voxel-based MRI study of volumetric differences and intercorrelations in autism. [Research Support, Non-U.S. Gov't]. Brain, 128(Pt 2), 268-276. doi: 10.1093/brain/awh332

McAlonan, G. M., Daly, E., Kumari, V., Critchley, H. D., van Amelsvoort, T., Suckling, J., . . Murphy, D. G. (2002). Brain anatomy and sensorimotor gating in Asperger's syndrome. Brain, 125(Pt 7), 1594-1606.

McAuley, T., Chen, S., Goos, L., Schachar, R., \& Crosbie, J. (2010). Is the behavior rating inventory of executive function more strongly associated with measures of impairment or executive function? J Int Neuropsychol Soc, 16(3), 495-505. doi: 10.1017/S1355617710000093

McGonigle-Chalmers, M., Bodner, K., Fox-Pitt, A., \& Nicholson, L. (2008). Size sequencing as a window on executive control in children with autism and Asperger's syndrome. J Autism Dev Disord, 38(7), 1382-1390. doi: 10.1007/s10803-007-0396-y

McNab, F., \& Klingberg, T. (2008). Prefrontal cortex and basal ganglia control access to working memory. Nat Neurosci, 11(1), 103-107. doi: 10.1038/nn2024

McQuarrie, A. D. R., \& Tsai, C. L. (1998). Regression and time series model selection. Singapore: World Scientific.

Menon, V., \& Uddin, L. Q. (2010). Saliency, switching, attention and control: a network model of insula function. [Research Support, N.I.H., Extramural Research Support, Non-U.S. Gov't Research Support, U.S. Gov't, Non-P.H.S. Review]. Brain Struct Funct, 214(5-6), 655-667. doi: 10.1007/s00429-010-0262-0

Mescher, M., Merkle, H., Kirsch, J., Garwood, M., \& Gruetter, R. (1998). Simultaneous in vivo spectral editing and water suppression. [Research Support, U.S. Gov't, P.H.S.]. NMR Biomed, 11(6), 266-272.

Miller, E. K., \& Cohen, J. D. (2001). An integrative theory of prefrontal cortex function. Annu Rev Neurosci, 24, 167-202. doi: 10.1146/annurev.neuro.24.1.167

Miller, G. A., \& Chapman, J. P. (2001). Misunderstanding analysis of covariance. J Abnorm Psychol, 110(1), 40-48.

Miyake, A., Friedman, N. P., Emerson, M. J., Witzki, A. H., Howerter, A., \& Wager, T. D. (2000). The unity and diversity of executive functions and their contributions to complex "Frontal Lobe" tasks: a latent variable analysis. Cogn Psychol, 41(1), 49-100. doi: 10.1006/cogp.1999.0734

Monk, C. S., Peltier, S. J., Wiggins, J. L., Weng, S. J., Carrasco, M., Risi, S., \& Lord, C. (2009). Abnormalities of intrinsic functional connectivity in autism spectrum disorders. [Research Support, N.I.H., Extramural]. Neuroimage, 47(2), 764-772. doi: 10.1016/j.neuroimage.2009.04.069

Montagne, B., Kessels, R. P., De Haan, E. H., \& Perrett, D. I. (2007). The Emotion Recognition Task: a paradigm to measure the perception of facial emotional expressions at different intensities. Percept Mot Skills, 104(2), 589-598.

Morf, M., Vieira, A., Lee, D. T. L., \& Keilath, T. (1978). Recursive Multichannel Maximum Entropy Spectral Estimation. IEEE Trans Geosci Electron, 16(2), 85-94.

Mountcastle, V. B. (1997). The columnar organization of the neocortex. [Review]. Brain, 120 (Pt 4), 701-722.

Muller, R. A., Shih, P., Keehn, B., Deyoe, J. R., Leyden, K. M., \& Shukla, D. K. (2011). Underconnected, but how? A survey of functional connectivity MRI studies in autism spectrum disorders. [Comparative Study Research Support, N.I.H., Extramural Review]. Cereb Cortex, 21(10), 2233-2243. doi: 10.1093/cercor/ bhq296 
Murray, D. M., Varnell, S. P., \& Blitstein, J. L. (2004). Design and analysis of group-randomized trials: A review of recent methodological developments. American Journal of Public Health, 94(3), 423-432. doi: Doi 10.2105/Ajph.94.3.423

Neill, J. J., \& Dunn, O. J. (1975). Equality of Dependent Correlation-Coefficients. Biometrics, 31(2), 531-543. doi: Doi 10.2307/2529435

Nelson, E. E., \& Guyer, A. E. (2011). The development of the ventral prefrontal cortex and social flexibility. Dev Cogn Neurosci, 1(3), 233-245. doi: 10.1016/j.dcn.2011.01.002

Nelson, E. E., Leibenluft, E., McClure, E. B., \& Pine, D. S. (2005). The social re-orientation of adolescence: a neuroscience perspective on the process and its relation to psychopathology. Psychological Medicine, 35(2), 163-174. doi: 10.1017/S0033291704003915

Neumann, D., Spezio, M. L., Piven, J., \& Adolphs, R. (2006). Looking you in the mouth: abnormal gaze in autism resulting from impaired top-down modulation of visual attention. [Research Support, Non-U.S. Gov't]. Soc Cogn Affect Neurosci, 1(3), 194-202. doi: 10.1093/scan/nsI030

Nichols, T. E., \& Holmes, A. P. (2002). Nonparametric permutation tests for functional neuroimaging: a primer with examples. [Research Support, Non-U.S. Gov't]. Hum Brain Mapp, 15(1), 1-25.

Nicpon, M. F., Doobay, A. F., \& Assouline, S. G. (2010). Parent, Teacher, and Self Perceptions of Psychosocial Functioning in Intellectually Gifted Children and Adolescents with Autism Spectrum Disorder. J Autism Dev Disord, 40(8), 1028-1038. doi: DOI 10.1007/s10803-010-0952-8

Nomi, J. S., \& Uddin, L. Q. (2015). Developmental changes in large-scale network connectivity in autism. [Research Support, N.I.H., Extramural]. Neuroimage Clin, 7, 732-741. doi: 10.1016/j.nicl.2015.02.024

Nordahl, C. W., Dierker, D., Mostafavi, I., Schumann, C. M., Rivera, S. M., Amaral, D. G., \& Van Essen, D. C. (2007). Cortical folding abnormalities in autism revealed by surface-based morphometry. J Neurosci, 27(43), 11725-11735. doi: 10.1523/JNEUROSCI.0777-07.2007

O'Hare, A. (2009). Autism spectrum disorder: diagnosis and management. Arch Dis Child Educ Pract Ed, 94(6), 161-168. doi: 10.1136/adc.2008.150490

O'Hare, E. D., Lu, L. H., Houston, S. M., Bookheimer, S. Y., \& Sowell, E. R. (2008). Neurodevelopmental changes in verbal working memory load-dependency: an fMRI investigation. Neuroimage, 42(4), 1678-1685. doi: 10.1016/j.neuroimage.2008.05.057

O'Hearn, K., Asato, M., Ordaz, S., \& Luna, B. (2008). Neurodevelopment and executive function in autism. Dev Psychopathol, 20(4), 1103-1132. doi: 10.1017/S0954579408000527

Olesen, P. J., Nagy, Z., Westerberg, H., \& Klingberg, T. (2003). Combined analysis of DTI and fMRI data reveals a joint maturation of white and grey matter in a fronto-parietal network. Brain Res Cogn Brain Res, 18(1), 48-57. doi: S092664100300212X [pii]

Olson, I. R., Plotzker, A., \& Ezzyat, Y. (2007). The Enigmatic temporal pole: a review of findings on social and emotional processing. [Review]. Brain, 130(Pt 7), 1718-1731. doi: 10.1093/brain/awm052

Orekhova, E. V., Stroganova, T. A., Prokofyev, A. O., Nygren, G., Gillberg, C., \& Elam, M. (2008). Sensory gating in young children with autism: relation to age, IQ, and EEG gamma oscillations. [Research Support, Non-U.S. Gov't]. Neurosci Lett, 434(2), 218-223. doi: 10.1016/j.neulet.2008.01.066

Osaka, N., Osaka, M., Kondo, H., Morishita, M., Fukuyama, H., \& Shibasaki, H. (2004). The neural basis of executive function in working memory: an fMRI study based on individual differences. Neuroimage, 21(2), 623-631. doi: 10.1016/j.neuroimage.2003.09.069

Owen, A. M., McMillan, K. M., Laird, A. R., \& Bullmore, E. (2005). N-back working memory paradigm: a meta-analysis of normative functional neuroimaging studies. Hum Brain Mapp, 25(1), 46-59. doi: 10.1002/hbm.20131

Ozonoff, S., \& Miller, J. N. (1995). Teaching theory of mind: a new approach to social skills training for individuals with autism. [Research Support, Non-U.S. Gov't]. J Autism Dev Disord, 25(4), 415-433.

Ozonoff, S., Pennington, B. F., \& Rogers, S. J. (1991). Executive function deficits in high-functioning autistic individuals: relationship to theory of mind. J Child Psychol Psychiatry, 32(7), 1081-1105.

Ozonoff, S., \& Strayer, D. (2001). Further evidence of intact working memory in autism. J Autism Dev Disord, 31(3), 257-263.

Palmer, S. E. (2002). Vision Science - Photons to Phenomenology. Cambridge, MA: MIT press. 
Paus, T. (2005). Mapping brain maturation and cognitive development during adolescence. Trends Cogn Sci, 9(2), 60-68. doi: 10.1016/j.tics.2004.12.008

Paus, T., Keshavan, M., \& Giedd, J. (2008). Why do many psychiatric disorders emerge during adolescence? Nat Rev Neurosci, 9(12), 947-957. doi: 10.1038/nrn2513

Pellicano, C. (2013). Sensory Symptoms in Autism: A Blooming, Buzzing Confusion? Child Development Perspectives, 7(3), 143-148.

Pellicano, E. (2010a). The development of core cognitive skills in autism: a 3-year prospective study. [Research Support, Non-U.S. Gov't]. Child Dev, 81(5), 1400-1416. doi: 10.1111/j.1467-8624.2010.01481.x

Pellicano, E. (2010b). Individual differences in executive function and central coherence predict developmental changes in theory of mind in autism. [Research Support, Non-U.S. Gov't]. Dev Psychol, 46(2), 530-544. doi: 10.1037/a0018287

Pennington, B. F., \& Ozonoff, S. (1996). Executive functions and developmental psychopathology. J Child Psychol Psychiatry, 37(1), 51-87.

Perlstein, W. M., Elbert, T., \& Stenger, V. A. (2002). Dissociation in human prefrontal cortex of affective influences on working memory-related activity. Proc Natl Acad Sci U S A, 99(3), 1736-1741. doi: 10.1073/ pnas. 241650598

Perner, J., \& Wimmer, H. (1985). John Thinks That Mary Thinks That - Attribution of 2nd-Order Beliefs by 5-Year-Old to 10-Year-Old Children. J Exp Child Psychol, 39(3), 437-471. doi: Doi 10.1016/0022-0965(85)90051-7

Pfeifer, J. H., \& Allen, N. B. (2012). Arrested development? Reconsidering dual-systems models of brain function in adolescence and disorders. Trends Cogn Sci, 16(6), 322-329. doi: 10.1016/j.tics.2012.04.011

Phillips, L. H., Channon, S., Tunstall, M., Hedenstrom, A., \& Lyons, K. (2008). The role of working memory in decoding emotions. Emotion, 8(2), 184-191. doi: 10.1037/1528-3542.8.2.184

Plaisted, K., Dobler, V., Bell, S., \& Davis, G. (2006). The microgenesis of global perception in autism. J Autism Dev Disord, 36(1), 107-116. doi: 10.1007/s10803-005-0047-0

Premack, D., \& Woodruff, G. (1978). Does the chimpanzee have a theory of mind? The Behavioral and Brain Sciences, 4, 515-526.

Pugliese, C. E., Anthony, L. G., Strang, J. F., Dudley, K., Wallace, G. L., Naiman, D. Q., \& Kenworthy, L. (2016). Longitudinal Examination of Adaptive Behavior in Autism Spectrum Disorders: Influence of Executive Function. [Research Support, N.I.H., Intramural Research Support, Non-U.S. Gov't]. J Autism Dev Disord, 46(2), 467-477. doi: 10.1007/s10803-015-2584-5

Puts, N. A., \& Edden, R. A. (2012). In vivo magnetic resonance spectroscopy of GABA: a methodological review. [Research Support, N.I.H., Extramural Review]. Prog Nucl Magn Reson Spectrosc, 60, 29-41. doi: 10.1016/j.pnmrs.2011.06.001

Rane, P., Cochran, D., Hodge, S. M., Haselgrove, C., Kennedy, D. N., \& Frazier, J. A. (2015). Connectivity in Autism: A Review of MRI Connectivity Studies. Harv Rev Psychiatry, 23(4), 223-244. doi: 10.1097/ HRP.0000000000000072

Redcay, E. (2008). The superior temporal sulcus performs a common function for social and speech perception: implications for the emergence of autism. [Review]. Neurosci Biobehav Rev, 32(1), 123-142. doi: 10.1016/j.neubiorev.2007.06.004

Robinson, S., Goddard, L., Dritschel, B., Wisley, M., \& Howlin, P. (2009). Executive functions in children with autism spectrum disorders. Brain Cogn, 71(3), 362-368. doi: 10.1016/j.bandc.2009.06.007

Roebroeck, A., Formisano, E., \& Goebel, R. (2005). Mapping directed influence over the brain using Granger causality and fMRI. Neuroimage, 25(1), 230-242. doi: 10.1016/j.neuroimage.2004.11.017

Rojas, D. C., Singel, D., Steinmetz, S., Hepburn, S., \& Brown, M. S. (2014). Decreased left perisylvian GABA concentration in children with autism and unaffected siblings. [Comparative Study Research Support, N.I.H., Extramural Review]. Neuroimage, 86, 28-34. doi: 10.1016/j.neuroimage.2013.01.045

Romero-Munguía, M. A. (2011). Mnesic Imbalance and the Neuroanatomy of Autism Spectrum Disorders. In V. Eapen (Ed.), Autism - A neurodevelopmental Journey from Genes to Behaviour (pp. 425-444). Rijeka: InTech.

Roth, J. K., \& Courtney, S. M. (2007). Neural system for updating object working memory from different sources: sensory stimuli or long-term memory. Neuroimage, 38(3), 617-630. doi: 10.1016/j.neuroimage. 2007.06.037 
Rubenstein, J. L., \& Merzenich, M. M. (2003). Model of autism: increased ratio of excitation/inhibition in key neural systems. [Research Support, Non-U.S. Gov't Research Support, U.S. Gov't, P.H.S. Review]. Genes Brain Behav, 2(5), 255-267.

Rubinov, M., \& Sporns, O. (2010). Complex network measures of brain connectivity: Uses and interpretations. Neuroimage, 52(3), 1059-1069. doi: DOI 10.1016/j.neuroimage.2009.10.003

Rudie, J. D., Brown, J. A., Beck-Pancer, D., Hernandez, L. M., Dennis, E. L., Thompson, P. M., . . Dapretto, M. (2012). Altered functional and structural brain network organization in autism. Neuroimage Clin, 2, 79-94. doi: 10.1016/j.nicl.2012.11.006

Rump, K. M., Giovannelli, J. L., Minshew, N. J., \& Strauss, M. S. (2009). The development of emotion recognition in individuals with autism. [Research Support, N.I.H., Extramural Research Support, Non-U.S. Gov't]. Child Dev, 80(5), 1434-1447. doi: 10.1111/j.1467-8624.2009.01343.x

Russell, J., \& Hill, E. L. (2001). Action-monitoring and intention reporting in children with autism. J Child Psychol Psychiatry, 42(3), 317-328.

Salter, G., Seigal, A., Claxton, M., Lawrence, K., \& Skuse, D. (2008). Can autistic children read the mind of an animated triangle? Autism, 12(4), 349-371. doi: 10.1177/1362361308091654

Schaefer, A., Braver, T. S., Reynolds, J. R., Burgess, G. C., Yarkoni, T., \& Gray, J. R. (2006). Individual differences in amygdala activity predict response speed during working memory. J Neurosci, 26(40), 10120-10128. doi: 10.1523/JNEUROSCI.2567-06.2006

Schaefer, G. B., \& Lutz, R. E. (2006). Diagnostic yield in the clinical genetic evaluation of autism spectrum disorders. Genet Med, 8(9), 549-556. doi: 10.109701.gim.0000237789.98842.f1

Scheeren, A. M., de Rosnay, M., Koot, H. M., \& Begeer, S. (2013). Rethinking theory of mind in high-functioning autism spectrum disorder. [Research Support, Non-U.S. Gov't]. J Child Psychol Psychiatry, 54(6), 628-635. doi: 10.1111/jcpp.12007

Schultz, R. T. (2005). Developmental deficits in social perception in autism: the role of the amygdala and fusiform face area. International Journal of Developmental Neuroscience, 23(2-3), 125-141. doi: DOI 10.1016/j.ijdevneu.2004.12.012

Seltzer, M. M., Shattuck, P., Abbeduto, L., \& Greenberg, J. S. (2004). Trajectory of development in adolescents and adults with autism. [Research Support, U.S. Gov't, P.H.S. Review]. Ment Retard Dev Disabil Res Rev, 10(4), 234-247. doi: 10.1002/mrdd.20038

Senju, A. (2013). Atypical development of spontaneous social cognition in autism spectrum disorders. [Research Support, Non-U.S. Gov't Review]. Brain Dev, 35(2), 96-101. doi: 10.1016/j.braindev.2012.08.002

Senju, A., Southgate, V., White, S., \& Frith, U. (2009). Mindblind eyes: an absence of spontaneous theory of mind in Asperger syndrome. [Research Support, Non-U.S. Gov't]. Science, 325(5942), 883-885. doi: 10.1126/science. 1176170

Seth, A. K. (2010). A MATLAB toolbox for Granger causal connectivity analysis. [Research Support, Non-U.S. Gov't]. J Neurosci Methods, 186(2), 262-273. doi: 10.1016/j.jneumeth.2009.11.020

Seth, A. K., Chorley, P., \& Barnett, L. C. (2013). Granger causality analysis of fMRI BOLD signals is invariant to hemodynamic convolution but not downsampling. [Research Support, Non-U.S. Gov't]. Neuroimage, 65, 540-555. doi: 10.1016/j.neuroimage.2012.09.049

Shattuck, P. T., Seltzer, M. M., Greenberg, J. S., Orsmond, G. I., Bolt, D., Kring, S., . . Lord, C. (2007). Change in autism symptoms and maladaptive behaviors in adolescents and adults with an autism spectrum disorder. [Research Support, N.I.H., Extramural]. J Autism Dev Disord, 37(9), 1735-1747. doi: 10.1007/ s10803-006-0307-7

Sheldrick, R. C., Neger, E. N., Shipman, D., \& Perrin, E. C. (2012). Quality of life of adolescents with autism spectrum disorders: concordance among adolescents' self-reports, parents' reports, and parents' proxy reports. Qual Life Res, 21(1), 53-57. doi: 10.1007/s11136-011-9916-5

Shukla, D. K., Keehn, B., \& Müller, R. A. (2011). Tract-specific analyses of diffusion tensor imaging show widespread white matter compromise in autism spectrum disorder. J Child Psychol Psychiatry, 52(3), 286-295. doi: 10.1111/j.1469-7610.2010.02342.x

Shukla, D. K., Keehn, B., Smylie, D. M., \& Müller, R. A. (2011). Microstructural abnormalities of short-distance white matter tracts in autism spectrum disorder. Neuropsychologia, 49(5), 1378-1382. doi: 10.1016/j.neuropsychologia.2011.02.022 
Sikora, D. M., Hall, T. A., Hartley, S. L., Gerrard-Morris, A. E., \& Cagle, S. (2008). Does parent report of behavior differ across ADOS-G classifications: analysis of scores from the CBCL and GARS. J Autism Dev Disord, 38(3), 440-448. doi: 10.1007/s10803-007-0407-z

Silk, T. J., Rinehart, N., Bradshaw, J. L., Tonge, B., Egan, G., O’Boyle, M. W., \& Cunnington, R. (2006). Visuospatial processing and the function of prefrontal-parietal networks in autism spectrum disorders: a functional MRI study. Am J Psychiatry, 163(8), 1440-1443. doi: 10.1176/appi.ajp.163.8.1440

Simpkins, C. A., \& Simpkins, A. M. (2013). Neurons and Neeurotransmitters Neuroscience for Clinicians (pp. 77-91). New York, NY: Springer New York.

Sinzig, J., Morsch, D., Bruning, N., Schmidt, M. H., \& Lehmkuhl, G. (2008). Inhibition, flexibility, working memory and planning in autism spectrum disorders with and without comorbid ADHD-symptoms. Child Adolesc Psychiatry Ment Health, 2(1), 4. doi: 10.1186/1753-2000-2-4

Smith, M. J. L., Montagne, B., Perrett, D. I., Gill, M., \& Gallagher, L. (2010). Detecting subtle facial emotion recognition deficits in high-functioning Autism using dynamic stimuli of varying intensities. Neuropsychologia, 48(9), 2777-2781. doi: DOI 10.1016/j.neuropsychologia.2010.03.008

Smith, S. M., Fox, P. T., Miller, K. L., Glahn, D. C., Fox, P. M., Mackay, C. E., . . Beckmann, C. F. (2009). Correspondence of the brain's functional architecture during activation and rest. [Research Support, N.I.H., Extramural Research Support, Non-U.S. Gov't]. Proc Natl Acad Sci U S A, 106(31), 13040-13045. doi: 10.1073/pnas.0905267106

Smith, S. M., \& Nichols, T. E. (2009). Threshold-free cluster enhancement: addressing problems of smoothing, threshold dependence and localisation in cluster inference. [Research Support, Non-U.S. Gov't]. Neuroimage, 44(1), 83-98. doi: 10.1016/j.neuroimage.2008.03.061

Snijders, T. M., Milivojevic, B., \& Kemner, C. (2013). Atypical excitation-inhibition balance in autism captured by the gamma response to contextual modulation. Neuroimage Clin, 3, 65-72. doi: 10.1016/j.nicl.2013.06.015

So, P., Greaves-Lord, K., Van der Ende, J., Verhulst, F. C., Rescorla, L., \& de Nijs, P. F. (2012). Using the Child Behavior Checklist and the Teacher's Report Form for identification of children with autism spectrum disorders. Autism. doi: 10.1177/1362361312448855

Soares, D. P., \& Law, M. (2009). Magnetic resonance spectroscopy of the brain: review of metabolites and clinical applications. [Review]. Clin Radiol, 64(1), 12-21. doi: 10.1016/j.crad.2008.07.002

Solomon, M., Ozonoff, S. J., Ursu, S., Ravizza, S., Cummings, N., Ly, S., \& Carter, C. S. (2009). The neural substrates of cognitive control deficits in autism spectrum disorders. Neuropsychologia, 47(12), 2515-2526. doi: 10.1016/j.neuropsychologia.2009.04.019

South, M., Schultz, R. T., \& Ozonoff, S. (2011). Social cognition in ASD. In D. Fein (Ed.), The neuropsychology of autism (pp. 225-242). New York: Oxford University Press.

Steele, S. D., Minshew, N. J., Luna, B., \& Sweeney, J. A. (2007). Spatial working memory deficits in autism. J Autism Dev Disord, 37(4), 605-612. doi: 10.1007/s10803-006-0202-2

Steinberg, L. (2005). Cognitive and affective development in adolescence. Trends Cogn Sci, 9(2), 69-74. doi: 10.1016/j.tics.2004.12.005

Steinberg, L., \& Morris, A. S. (2001). Adolescent development. Annu Rev Psychol, 52, 83-110. doi: 10.1146/ annurev.psych.52.1.83

Stichter, J. P., Herzog, M. J., Visovsky, K., Schmidt, C., Randolph, J., Schultz, T., \& Gage, N. (2010). Social competence intervention for youth with Asperger Syndrome and high-functioning autism: an initial investigation. [Clinical Trial Research Support, Non-U.S. Gov't]. J Autism Dev Disord, 40(9), 1067-1079. doi: 10.1007/s10803-010-0959-1

Stone, V. E., \& Gerrans, P. (2006). What's domain-specific about theory of mind? Soc Neurosci, 1(3-4), 309-319. doi: 10.1080/17470910601029221

Sukhodolsky, D. G., Scahill, L., Gadow, K. D., Arnold, L. E., Aman, M. G., McDougle, C. J., . . Vitiello, B. (2008). Parent-rated anxiety symptoms in children with pervasive developmental disorders: frequency and association with core autism symptoms and cognitive functioning. J Abnorm Child Psychol, 36(1), 117-128. doi: 10.1007/s10802-007-9165-9

Swanson, H. L. (2004). Working memory and phonological processing as predictors of children's mathematical problem solving at different ages. Mem Cognit, 32(4), 648-661. 
Symeonidou, I., Dumontheil, I., Chow, W. Y., \& Breheny, R. (2016). Development of online use of theory of mind during adolescence: An eye-tracking study. J Exp Child Psychol, 149, 81-97. doi: 10.1016/j.jecp.2015.11.007

Takahama, S., Miyauchi, S., \& Saiki, J. (2010). Neural basis for dynamic updating of object representation in visual working memory. Neuroimage, 49(4), 3394-3403. doi: 10.1016/j.neuroimage.2009.11.029

Thomas, C. G., Harshman, R. A., \& Menon, R. S. (2002). Noise reduction in BOLD-based fMRI using component analysis. [Evaluation Studies Research Support, Non-U.S. Gov't]. Neuroimage, 17(3), $1521-1537$.

Tirouvanziam, R., Obukhanych, T. V., Laval, J., Aronov, P. A., Libove, R., Banerjee, A. G., . . Hardan, A. Y. (2012). Distinct plasma profile of polar neutral amino acids, leucine, and glutamate in children with Autism Spectrum Disorders. [Research Support, N.I.H., Extramural Research Support, Non-U.S. Gov't]. J Autism Dev Disord, 42(5), 827-836. doi: 10.1007/s10803-011-1314-x

Toplak, M. E., Bucciarelli, S. M., Jain, U., \& Tannock, R. (2009). Executive functions: performance-based measures and the behavior rating inventory of executive function (BRIEF) in adolescents with attention deficit/hyperactivity disorder (ADHD). Child Neuropsychol, 15(1), 53-72. doi: 10.1080/09297040802070929

Tracy, J. L., Robins, R. W., Schriber, R. A., \& Solomon, M. (2011). Is emotion recognition impaired in individuals with autism spectrum disorders? J Autism Dev Disord, 41(1), 102-109. doi: 10.1007/s10803-010-1030-y

Travers, B. G., Klinger, M. R., \& Klinger, L. G. (2011). Attention and working memory in ASD. In D. Fein (Ed.), The neuropsychology of autism (pp. 161-184). New York: Oxford University Press.

Treffers, P., D.,A., Goedhardt, A.W., Veerman, J.W., Van den bergh, B.R.H., Ackaert, L. \& de Rycke, L. (2002). Handleiding Competentie Belevingsschaal voor Adolescenten. Lisse: Swets Test Publishers.

Tu, W. J., Chen, H., \& He, J. (2012). Application of LC-MS/MS analysis of plasma amino acids profiles in children with autism. J Clin Biochem Nutr, 51(3), 248-249. doi: 10.3164/jcbn.12-45

Tyszka, J. M., Kennedy, D. P., Paul, L. K., \& Adolphs, R. (2014). Largely typical patterns of resting-state functional connectivity in high-functioning adults with autism. [Research Support, N.I.H., Extramural Research Support, Non-U.S. Gov't]. Cereb Cortex, 24(7), 1894-1905. doi: 10.1093/cercor/bht040

Uddin, L. Q., \& Menon, V. (2009). The anterior insula in autism: under-connected and under-examined. [Research Support, N.I.H., Extramural Research Support, Non-U.S. Gov't Research Support, U.S. Gov't, Non-P.H.S. Review]. Neurosci Biobehav Rev, 33(8), 1198-1203. doi: 10.1016/j.neubiorev.2009.06.002

Uddin, L. Q., Supekar, K., \& Menon, V. (2013). Reconceptualizing functional brain connectivity in autism from a developmental perspective. Front Hum Neurosci, 7, 458. doi: 10.3389/fnhum.2013.00458

Unsworth, N., Redick, T. S., Heitz, R. P., Broadway, J. M., \& Engle, R. W. (2009). Complex working memory span tasks and higher-order cognition: a latent-variable analysis of the relationship between processing and storage. Memory, 17(6), 635-654. doi: 10.1080/09658210902998047

Vaessen, M. J., Hofman, P. A., Tijssen, H. N., Aldenkamp, A. P., Jansen, J. F., \& Backes, W. H. (2010). The effect and reproducibility of different clinical DTI gradient sets on small world brain connectivity measures. Neuroimage, 51(3), 1106-1116. doi: 10.1016/j.neuroimage.2010.03.011

van den Bergh, S. F., Scheeren, A. M., Begeer, S., Koot, H. M., \& Geurts, H. M. (2014). Age related differences of executive functioning problems in everyday life of children and adolescents in the autism spectrum. [Research Support, Non-U.S. Gov't]. J Autism Dev Disord, 44(8), 1959-1971. doi: 10.1007/s10803-0142071-4

van der Ende, J., \& Verhulst, F. C. (2005). Informant, gender and age differences in ratings of adolescent problem behaviour. Eur Child Adolesc Psychiatry, 14(3), 117-126. doi: 10.1007/s00787-005-0438-y

van der Kruijs, S. J., Jagannathan, S. R., Bodde, N. M., Besseling, R. M., Lazeron, R. H., Vonck, K. E., . . . Jansen, J. F. (2014). Resting-state networks and dissociation in psychogenic non-epileptic seizures. J Psychiatr Res, 54, 126-133. doi: 10.1016/j.jpsychires.2014.03.010

Van Dijk, K. R., Hedden, T., Venkataraman, A., Evans, K. C., Lazar, S. W., \& Buckner, R. L. (2010). Intrinsic functional connectivity as a tool for human connectomics: theory, properties, and optimization. $J$ Neurophysiol, 103(1), 297-321. doi: 10.1152/jn.00783.2009

van Oosten, M. H. M., Oosterhoff, M. D., \& Kan, C. C. (2011). Diagnostiek van autismespectrumstoornissen bij volwassenen: een multidisciplinaire benadering. Amsterdam: Hogrefe. 
van Veenendaal, T. M., DM, I. J., Aldenkamp, A. P., Hofman, P. A., Vlooswijk, M. C., Rouhl, R. P., . . Jansen, J. F. (2015). Metabolic and functional MR biomarkers of antiepileptic drug effectiveness: A review. Neurosci Biobehav Rev, 59, 92-99. doi: 10.1016/j.neubiorev.2015.10.004

Vanhaudenhuyse, A., Noirhomme, Q., Tshibanda, L. J., Bruno, M. A., Boveroux, P., Schnakers, C., . . Boly, M. (2010). Default network connectivity reflects the level of consciousness in non-communicative brain-damaged patients. [Comparative Study Research Support, N.I.H., Extramural Research Support, Non-U.S. Gov't]. Brain, 133(Pt 1), 161-171. doi: 10.1093/brain/awp313

Verhulst, F. C., \& van der Ende, J. (1992). Agreement between parents' reports and adolescents' self-reports of problem behavior. [Research Support, Non-U.S. Gov't]. J Child Psychol Psychiatry, 33(6), 1011-1023.

Verté, S., Geurts, H. M., Roeyers, H., Oosterlaan, J., \& Sergeant, J. A. (2006a). Executive functioning in children with an Autism Spectrum Disorder: can we differentiate within the spectrum? J Autism Dev Disord, 36(3), 351-372. doi: 10.1007/s10803-006-0074-5

Verté, S., Geurts, H. M., Roeyers, H., Oosterlaan, J., \& Sergeant, J. A. (2006b). The relationship of working memory, inhibition, and response variability in child psychopathology. J Neurosci Methods, 151(1), 5-14. doi: 10.1016/j.jneumeth.2005.08.023

Vestergaard, M., Madsen, K. S., Baaré, W. F., Skimminge, A., Ejersbo, L. R., Ramsøy, T. Z., . . Jernigan, T. L. (2010). White Matter Microstructure in Superior Longitudinal Fasciculus Associated with Spatial Working Memory Performance in Children. J Cogn Neurosci. doi: 10.1162/jocn.2010.21592

Vlooswijk, M. C., Jansen, J. F., de Krom, M. C., Majoie, H. M., Hofman, P. A., Backes, W. H., \& Aldenkamp, A. P. (2010). Functional MRI in chronic epilepsy: associations with cognitive impairment. [Research Support, Non-U.S. Gov't Review]. Lancet Neurol, 9(10), 1018-1027. doi: 10.1016/S1474-4422(10)70180-0

Vollm, B. A., Taylor, A. N., Richardson, P., Corcoran, R., Stirling, J., McKie, S., . . Elliott, R. (2006). Neuronal correlates of theory of mind and empathy: a functional magnetic resonance imaging study in a nonverbal task. Neuroimage, 29(1), 90-98. doi: 10.1016/j.neuroimage.2005.07.022

Vriezen, E. R., \& Pigott, S. E. (2002). The relationship between parental report on the BRIEF and performancebased measures of executive function in children with moderate to severe traumatic brain injury. Child Neuropsychol, 8(4), 296-303. doi: 10.1076/chin.8.4.296.13505

Wallace, G. L., Case, L. K., Harms, M. B., Silvers, J. A., Kenworthy, L., \& Martin, A. (2011). Diminished sensitivity to sad facial expressions in high functioning autism spectrum disorders is associated with symptomatology and adaptive functioning. [Research Support, N.I.H., Intramural]. J Autism Dev Disord, 41(11), 1475-1486. doi: 10.1007/s10803-010-1170-0

Wallace, G. L., Dankner, N., Kenworthy, L., Giedd, J. N., \& Martin, A. (2010). Age-related temporal and parietal cortical thinning in autism spectrum disorders. Brain, 133(Pt 12), 3745-3754. doi: 10.1093/brain/awq279

Wang, X., Foryt, P., Ochs, R., Chung, J. H., Wu, Y., Parrish, T., \& Ragin, A. B. (2011). Abnormalities in resting-state functional connectivity in early human immunodeficiency virus infection. [Research Support, N.I.H., Extramural]. Brain Connect, 1(3), 207-217. doi: 10.1089/brain.2011.0016

Wechsler, D. (2005). Wechsler Intelligence Scale for Children - Third Edition (WISC-III) Manual. Amsterdam: Harcourt Test Publishers.

Weissman, D. H., Perkins, A. S., \& Woldorff, M. G. (2008). Cognitive control in social situations: a role for the dorsolateral prefrontal cortex. Neuroimage, 40(2), 955-962. doi: 10.1016/j.neuroimage.2007.12.021

White, S. W., Oswald, D., Ollendick, T., \& Scahill, L. (2009). Anxiety in children and adolescents with autism spectrum disorders. [Research Support, N.I.H., Extramural Review]. Clin Psychol Rev, 29(3), 216-229. doi: 10.1016/j.cpr.2009.01.003

White, S. W., \& Roberson-Nay, R. (2009). Anxiety, social deficits, and loneliness in youth with autism spectrum disorders. [Research Support, N.I.H., Extramural]. J Autism Dev Disord, 39(7), 1006-1013. doi: 10.1007/ s10803-009-0713-8

Wicker, B., Fonlupt, P., Hubert, B., Tardif, C., Gepner, B., \& Deruelle, C. (2008). Abnormal cerebral effective connectivity during explicit emotional processing in adults with autism spectrum disorder. [Research Support, Non-U.S. Gov't]. Soc Cogn Affect Neurosci, 3(2), 135-143. doi: 10.1093/scan/nsn007

Williams, D. L., Goldstein, G., Carpenter, P. A., \& Minshew, N. J. (2005). Verbal and spatial working memory in autism. J Autism Dev Disord, 35(6), 747-756. doi: 10.1007/s10803-005-0021-x 
Williams, D. L., Goldstein, G., \& Minshew, N. J. (2006). The profile of memory function in children with autism. Neuropsychology, 20(1), 21-29. doi: 10.1037/0894-4105.20.1.21

Wing, L. (1981). Asperger's syndrome: a clinical account. [Case Reports]. Psycho/ Med, 11(1), 115-129.

Wing, L. (1996). Autistic spectrum disorders. BMJ, 312(7027), 327-328.

Wing, L. (1997). The autistic spectrum. Lancet, 350(9093), 1761-1766. doi: 10.1016/S0140-6736(97)09218-0

Wing, L., \& Potter, D. (2002). The epidemiology of autistic spectrum disorders: is the prevalence rising? Ment Retard Dev Disabil Res Rev, 8(3), 151-161. doi: 10.1002/mrdd.10029

Wodka, E. L., \& Mostofsky, S. H. (2011). Motor development and its relation to social and behavioral manifestations in children with ASD. In D. Fein (Ed.), The neuropsychology of autism (pp. 205-214). New York: Oxford University Press.

Zaremba, A., \& Aste, T. (2014). Measures of Causality in Complex Datasets with Application to Financial Data. Entropy 16(4), 2309-2349. doi: 10.3390/e16042309

Zhou, Z., Wang, X., Klahr, N. J., Liu, W., Arias, D., Liu, H., . . Liu, Y. (2011). A conditional Granger causality model approach for group analysis in functional magnetic resonance imaging. [Research Support, N.I.H., Extramural Research Support, Non-U.S. Gov't]. Magn Reson Imaging, 29(3), 418-433. doi: 10.1016/j.mri.2010.10.008

Zikopoulos, B., \& Barbas, H. (2013). Altered neural connectivity in excitatory and inhibitory cortical circuits in autism. Front Hum Neurosci, 7, 609. doi: 10.3389/fnhum.2013.00609

Zinke, K., Fries, E., Altgassen, M., Kirschbaum, C., Dettenborn, L., \& Kliegel, M. (2010). Visuospatial short-term memory explains deficits in tower task planning in high-functioning children with autism spectrum disorder. Child Neuropsychol, 16(3), 229-241. doi: 10.1080/09297040903559648

Zuo, X. N., Kelly, C., Adelstein, J. S., Klein, D. F., Castellanos, F. X., \& Milham, M. P. (2010). Reliable intrinsic connectivity networks: test-retest evaluation using ICA and dual regression approach. [Research Support, N.I.H., Extramural Research Support, Non-U.S. Gov't]. Neuroimage, 49(3), 2163-2177. doi: 10.1016/j.neuroimage.2009.10.080 



\section{Dankwoord}

Na heel wat jaren werk en de nodige life-events is het proefschift af! Dit was zeker niet gelukt zonder de onvermoeibare inzet van anderen, die ik dan ook zeker wil bedanken:

Mijn dank gaat in de eerste plaats uit naar mijn promotoren en co-promotoren. Bert Aldenkamp, allereerst natuurlijk bedankt voor het aanbieden van deze promotieplek. Een unieke kans, waarvan ik heel erg veel heb geleerd en genoten. Door de jaren heen bleef je de lijn van het onderzoek en de artikelen goed bewaken en waarschuwde me wanneer ik weer teveel afweek van deze lijn (zoveel gerelateerde onderwerpen zijn óók interessant) of teveel in de details dook. Heel erg bedankt voor je adviezen en gesprekken; streng wanneer nodig, altijd motiverend en opbouwend. Ook gaf je mij de kans om wetenschap te blijven combineren met het ontwikkelen van mijn klinische vaardigheden, wat ik erg gewaardeerd heb. En natuurlijk niet te vergeten, bedankt voor de heerlijke etentjes bij van Gaalen! Roy Kessels; als buitenpromovendus gaf je mij een mooie plek binnen de afdeling neuro- en revalidatiepsychologie van de universiteit. Ondanks dat ik een buitenpromovendus was, heb ik mij nooit een buitenbeentje op de afdeling gevoeld en dat kwam zeker door de manier en vanzelfsprekendheid waarop jij mij hebt opgenomen in het team. Bedankt dat je me alle mogelijkheden gaf om te profiteren van de faciliteiten van de universiteit. Ondanks je drukke agenda, gaf je altijd snelle en zeer bruikbare feedback en kon ik altijd bij je binnenlopen voor advies of een praatje. Naast je wetenschappelijke bijdrage aan mijn ontwikkeling, zal ik ook zeker je invloed om mijn muzikale ontwikkeling niet vergeten (nacht der Professoren)! Marc Hendriks, jou ken ik al vanaf mijn tijd als student op de universiteit toen je mijn docent en stagebegeleider/ scriptiebegeleider was. Sinds mijn afstuderen heb je altijd veel interesse in mijn loopbaanontwikkeling getoond en jij was dan ook degene die mij attendeerde op de promotieplek op Kempenhaeghe. Sinds mijn afstuderen heb je mij altijd benaderd als een collega (ipv een student of jong ding). Je hebt mij gestimuleerd om zelfstandig te werken en om meer te vertrouwen te krijgen in mijn eigen handelen en kennis. Wel altijd met het idee dat ik op je terug kon vallen mocht het nodig zijn. Ook tijdens mijn promotietraject heb je mij gemotiveerd om mij zowel op wetenschappelijk als op klinisch gebied te blijven ontwikkelen (en mocht ik zelfs mee naar een Penfield operatie) en bleef je je kennis en ervaring met mij delen. Naast deze werkgerelateerde zaken, was je ook altijd erg betrokken bij mijn persoonlijke leven en verhalen. Ik heb onze gesprekken, waarin er altijd ruimte was om bij te praten, dan ook altijd erg gewaardeerd. Jaap Jansen, erg bedankt voor je inbreng van kennis en kunde op het gebied van de neuroscience en MRI technologie. Je was altijd bereid om dingen op een begrijpelijke manier uit te leggen, waardoor ik de link tussen onze bevindingen en de klinische praktijk beter kon leggen. Al mijn promotoren en co-promotoren wil ik tenslotte 
bedanken voor hun geduld; door mijzelf gelijktijdig te willen ontwikkelen op meerdere gebieden (als wetenschapper, psycholoog en als moeder) heeft het traject langer geduurd dan normaal, maar door het vertrouwen dat jullie mij gaven en de motiverende gesprekken, is het gelukt om deze drie, voor mij zeer belangrijke levensgebieden, succesvol te kunnen combineren.

Dit onderzoek werd (gedeeltelijk) gefinancierd door SVO de Berkenschutse. Ik wil alle medewerkers daar dan ook bedanken voor hun interesse, medewerking en feedback. Het was bijzonder leerzaam om mee te mogen kijken op jullie school! In het bijzonder wil ik nog Theo Laureijs en Geert Thoonen bedanken; Theo bedankt voor het openstellen van je school, onze prettige gesprekken, en het samen brainstormen. Geert, bedankt voor het delen van je kennis en kunde op het gebied van deze bijzonder (leuke) groep jongeren op deze school.

Mijn grote dank gaat natuurlijk ook uit naar alle deelnemers aan dit onderzoek: sommigen deden het 'voor de wetenschap', sommigen om een ochtendje school te missen, maar jullie inzet was hoe dan ook fantastisch! Verder wil ik natuurlijk ook Mariken Dinissen en Renée Kessels bedanken, die als masterstudenten hebben bijgedragen aan de dataverzameling voor dit proefschrift. Dank ook aan de co-auteurs van een deel van de artikelen in dit proefschrift, en in het speciaal dank aan Lisanne Schreuder, Antoine Bernass en Gerald Drenthen voor jullie inzet, het delen van jullie expertise en de fijne samenwerking. Mijn oud-collega's op de afdeling Neuro- en revalidatiepsychologie van de Radboud Universiteit en de afdelingen Onderzoek \& Ontwikkeling en de Gedragswetenschappelijke dienst van Kempenhaeghe wil ik niet alleen bedanken voor hun inhoudelijke feedback en de gezellige koffiepauzes, maar ook het luisterend oor dat ze hebben geboden als het even niet zo leuk meer was. Bedankt voor alle steun en de geweldige tijd samen! In het bijzonder wil ik mijn oud kamer en ganggenoten bedanken: Petra, Dirk, Johanna, Jessica, Heiko, Zita, Petra, en Egbert. En Bonnie, mijn kamergenoot en paranimf; niet alleen hebben wij ons samen door het promotietraject heen gewerkt, hebben we samen veel gelachen en soms ook gehuild, maar zijn we ook vlak na elkaar moeder geworden. Hoewel we nu geen directe collega's meer zijn, hebben we nog steeds contact en spelen onze zoontjes soms samen; ik had me geen betere kamergenoot kunnen wensen!

En omdat de boog niet altijd strak gespannen kan zijn, wil ik zeker ook mijn vrienden bedanken. Zij hebben altijd (voorzichtig) gevraagd hoe het ervoor stond, hebben mij gesteund en gemotiveerd om door te zetten, en hebben vooral ook voor de nodige afleiding gezorgd! Betties (Anouk, Christine, Hester en Marieke) en de mannen (lan, Michel en Thijs) bedankt! 
Lieve pa, ma, Silvia en Leonard, bedankt voor alle steun die jullie mij altijd hebben gegeven. Jullie waren mijn vaste fanclub, toonden altijd interesse in mijn vorderingen en hielpen daar waar nodig. Pa, ma en Stefa, bedankt voor alle extra oppasuurtjes waardoor het gelukt is om alles te blijven combineren en ik alles langzaam maar zeker toch kon afronden. En lieve Rob; altijd relativerend, steunend, de-escalerend :-) en inhoudelijk geïnteresseerd. Na heel wat jaren samen, zijn we nu beiden (vooralsnog) klaar met onze opleiding, en hebben we weer wat meer tijd samen. Ik ben ontzettend gek op je, trots op wat we samen hebben bereikt, en op onze lieve kinderen Fedde en Liselot! 



\section{Curriculum Vitae}

Evelien Maria Barendse was born on October $10^{\text {th }} 1980$ in Wageningen, the Netherlands. In 1999 she completed her higher scientific secondary education (VWO) at the Hendrik Pearson College in Zetten. The same year she moved to Breda to study Graphic Design at the Art Academy St. Joost. In 2000 she decided that after all, being creative was more like a hobby to her, so she moved to Nijmegen to study Psychology at the Katholieke Universiteit Nijmegen. After obtaining her master degree in Neuro- and Rehabilitation psychology, she started working part-time as a Psychological Assistant at Karakter Nijmegen, a centre for Child and adolescent psychiatry. At the same time, she started working as a pediatric neuropsychologist at Sint Marie in Eindhoven, a centre for children and adolescents with language, speech and communication difficulties. During her studies, her interest in scientific research grew, and she got involved as a research assistant in several studies. During her clinical work, this interest was further strengthened. This was one of the reasons why, when she was asked if she was interested in participating in a PhD program in 2009, she did not have to think twice. In 2009 she started the $\mathrm{PhD}$ research described in this thesis. Nevertheless, over the years she kept her interest in the clinical work as well and in January 2014, she enrolled the Postdoc Education towards Clinical Psychologist at the Psychiatry department of the Radboud University Medical Centre in Nijmegen. At 13 February 2014 their son Fedde was born. In May 2016, Evelien obtained her title as GZ-psychologist, and started working until January 2017 as a GZ-psychologist at the Medical psychology department of Viecuri Medical Centre in Venlo. At 27 March 2017 their daughter Liselot was born, and later that year Evelien finished her thesis. At present Evelien Barendse is back working in Nijmegen as a GZ-psychologist at the Audiological Centre of the Radboud UMC.

Evelien Maria Barendse is geboren op 10 oktober 1980 in Wagening. In 1999 behaalde ze haar VWO diploma aan het Hendrik Pearson College in Zetten. In datzelfde jaar verhuisde ze naar Breda om daar Grafisch Ontwerp te studeren op de kunstacademie St. Joost. In 2000 besloot ze dat creatief zijn toch meer een hobby voor haar was, en verhuisde ze naar Nijmegen om Psychologie te gaan studeren aan de Katholieke Universiteit Nijmegen. Na het behalen van haar doctorandus titel in de neuro- en revalidatiepsychologie, ging ze werken als psychologisch assistent bij Karakter Nijmegen, een centrum voor kinder- en jeugd psychiatrie. Tegelijkertijd begon ze als kinderneuropsycholoog te werken bij Sint Marie in Eindhoven, een centrum voor kinderen en adolescenten met taal-, spraak-, en communicatieproblemen. De interesse in het wetenschappelijk onderzoek werd tijdens haar opleiding al gewekt en ze was dan ook als onderzoeksassistent betrokken bij verschillende onderzoeken. Na haar studie en tijdens haar klinische werkzaamheden werd deze interesse verder versterkt. 
Dit leidde er mede toe dat, toen haar in 2009 werd gevraagd of ze interesse had in een promotietraject, ze niet lang hoefde na te denken. In 2009 startte ze met het met het promotieonderzoek beschreven in dit proefschrift. Toch bleef ook haar interesse in het klinische werk bestaan en in januari 2014 startte zij dan ook met haar opleiding tot GZ-psycholoog bij het Radboud UMC, afdeling psychiatrie. Op 13 februari 2014 werd hun zoon Fedde geboren. In mei 2016 verkreeg Evelien haar titel als GZpsycholoog, en werkte tot Januari 2017 als GZ-psycholoog op de afdeling Medische psychologie van het Viecuri medisch centrum te Venlo. Op 27 maart 2017 is hun dochter Liselot geboren en later dat jaar heeft zij haar proefschrift afgerond. Op dit moment werkt Evelien Barendse weer in Nijmegen als GZ-psycholoog op het Audiologisch Centrum van het Radboud UMC. 


\section{Donders Graduate School for Cognitive Neuroscience}

For a successful research Institute, it is vital to train the next generation of young scientists. To achieve this goal, the Donders Institute for Brain, Cognition and Behaviour established the Donders Graduate School for Cognitive Neuroscience (DGCN), which was officially recognised as a national graduate school in 2009. The Graduate School covers training at both Master's and PhD level and provides an excellent educational context fully aligned with the research programme of the Donders Institute.

The school successfully attracts highly talented national and international students in biology, physics, psycholinguistics, psychology, behavioral science, medicine and related disciplines. Selective admission and assessment centers guarantee the enrolment of the best and most motivated students.

The DGCN tracks the career of PhD graduates carefully. More than $50 \%$ of $\mathrm{PhD}$ alumni show a continuation in academia with postdoc positions at top institutes worldwide, e.g. Stanford University, University of Oxford, University of Cambridge, UCL London, MPI Leipzig, Hanyang University in South Korea, NTNU Norway, University of Illinois, North Western University, Northeastern University in Boston, ETH Zürich, University of Vienna etc.. Positions outside academia spread among the following sectors: specialists in a medical environment, mainly in genetics, geriatrics, psychiatry and neurology. Specialists in a psychological environment, e.g. as specialist in neuropsychology, psychological diagnostics or therapy. Positions in higher education as coordinators or lecturers. A smaller percentage enters business as research consultants, analysts or head of research and development. Fewer graduates stay in a research environment as lab coordinators, technical support or policy advisors. Upcoming possibilities are positions in the IT sector and management position in pharmaceutical industry. In general, the PhDs graduates almost invariably continue with high-quality positions that play an important role in our knowledge economy.

For more information on the DGCN as well as past and upcoming defenses please visit: http://www.ru.nl/donders/graduate-school/phd/ 